\title{
CHARACTERIZATION OF MULTIPLE TRANSVERSE FRACTURE WELLS USING THE ASYMPTOTIC APPROXIMATION OF THE DIFFUSIVITY EQUATION
}

\author{
A Thesis \\ by \\ ANDREW DAVID MALONE \\ Submitted to the Office of Graduate and Professional Studies of \\ Texas A\&M University \\ in partial fulfillment of the requirements for the degree of \\ MASTER OF SCIENCE
}

Chair of Committee, Michael J. King

Committee Members, David Schechter

Juan Carlos Laya

Head of Department, Jeff Spath

May 2019

Major Subject: Petroleum Engineering

Copyright 2019 Andrew David Malone 


\begin{abstract}
Shale oil and gas remains a developing field of study because of the inherent complexities of producing fluids from ultra-tight media via wells with highly complex, uncertain geometries. Production analysis provides evaluators with powerful tools for the optimization of production from unconventional reservoirs, requiring only limited geologic and petrophysical data, given that certain assumptions are made. In this study, we present a new subset of analytic formulations for the production analysis of Multiple Transverse Fracture Wells (MTFW's). These analytic models are based upon the asymptotic approximation of the diffusivity equation and are unique in that they apply the asymptotic solution in conjunction with the principle of superposition to represent the interactions between hydraulic fractures during reservoir drainage from MTFW's. With these models we show that the interaction between hydraulic fractures during MTFW production will appear similar to boundary-dominated flow (BDF) on a diagnostic plot but is in fact a unique interference signature that results from the superposition of pressure drawdowns induced upon each fracture by its neighbors.

In addition, we present our work on the explicit definition of the end of transient linear flow in MTFW's. Previous authors have observed and defined the "end of linear flow", however, we discovered that this definition remains ambiguous in industry literature. We present our own definition of the end of linear flow, based upon the core construction of the asymptotic approximation of the diffusivity equation, founded upon the concept of detectability. Our definition, dubbed the "limit of detectability" is
\end{abstract}


intuitive, recognizable on classic pressure transient diagnostic and specialized plots, and independent of inner boundary conditions. 


\section{DEDICATION}

This work is dedicated to my wonderful wife, Hallie Malone, who stood by me and supported me through my nearly three years of graduate school. 


\section{ACKNOWLEDGEMENTS}

I extend my gratitude towards my research advisor, Dr. Michael J. King, for supporting and mentoring me through my graduate studies. Dr. King gave me a fantastic opportunity to seek out a better life for myself and my family during what was arguably one of the most difficult times our industry has ever seen.

I thank my committee members, Dr. David Schechter and Dr. Juan-Carlos Laya for their guidance, support, and encouragement as well.

I would also like to thank my former fellow student, Dr. Zhenzhen Wang, for mentoring me as I worked on this project, as well as the other unnamed members of the MCERI research group for their friendship through my graduate years. Working with such a strong, capable team certainly drove me to better myself, and I have them to thank for that.

Finally, I thank my parents for teaching me that you can always exceed your own expectations as long as you try. 


\section{CONTRIBUTORS AND FUNDING SOURCES}

\section{Contributors}

This work was supervised by a thesis committee consisting of Professors Michael J. King and David Schechter of the Department of Petroleum Engineering and Professor Juan-Carlos Laya of the Department of Geology.

The work done on the Montney field case (presented in Chapter III) was performed in collaboration with Zhenzhen Wang and was published in 2018.

All other work in this thesis was completed independently by the student.

\section{Funding Sources}

This work was made possible by the financial support of Energi Simulation (formerly Foundation CMG) through the Texas A\&M chair in Robust Reduced Complexity Modeling with Dr. Eduardo Gildin and the members of the Model Calibration and Efficient Reservoir imaging (MCERI) joint industry project at Texas A\&M University.

Graduate study was also supported by a fellowship from Texas A\&M University. 


\section{NOMENCLATURE}

Abbreviations

$\begin{array}{ll}\text { APSS } & \text { Approximate Pseudo Steady State } \\ \text { BHP } & \text { Bottom Hole Pressure } \\ \text { BI } & \text { Brittleness Index } \\ \text { DOI } & \text { Depth of Investigation } \\ \text { EOD } & \text { Environment of Deposition } \\ \text { EDFM } & \text { Embedded Discrete Fracture Model } \\ \text { FPM } & \text { Fracture Propagation Model } \\ \text { HF } & \text { Hydraulic Fracture } \\ \text { IALF } & \text { Infinite-acting Linear Flow } \\ \text { IAPF } & \text { Infinite-acting Pillbox Flow } \\ \text { OHC } & \text { Original Oil in Place } \\ \text { OHFer Boundary Condition } \\ \text { LEF }\end{array}$




$\begin{array}{ll}\text { PEBI } & \text { Perpendicular Bisector } \\ \text { PPSS } & \text { Pseudo Pseudo Steady State } \\ \text { PSS } & \text { Pseudo Steady State } \\ \text { PTA } & \text { Pressure Transient Analysis } \\ \text { RTA } & \text { Rate Transient Analysis } \\ \text { SEM } & \text { Scanning Electron Microscope } \\ \text { SRV } & \text { Stimulated Reservoir Volume } \\ \text { TOC } & \text { Total Organic Content } \\ \text { UEF } & \text { Upper Eagle Ford }\end{array}$

Alphabetic Symbols

$\begin{array}{ll}A & \text { Cross-sectional flow area } \\ A_{n} & \text { Unknown function } \\ B & \text { Formation volume factor } \\ C_{r D} & \text { Dimensionless fracture conductivity } \\ c_{t} & \text { Total compressibility } \\ h & \text { Reservoir height } \\ k & \text { Permeability } \\ L_{w} & \text { Horizontal well lateral section length } \\ n_{f} & \text { Number of fractures } \\ N_{p} & \text { Cumulative oil production }\end{array}$




\begin{tabular}{|c|c|}
\hline$p$ & Pressure \\
\hline$p_{w D}$ & Dimensionless bottom hole pressure \\
\hline$p_{w f}$ & Bottom hole flowing pressure \\
\hline$q$ & Flux \\
\hline$q_{D}$ & Dimensionless flow rate \\
\hline$q_{w}$ & Surface flux (flow rate at the well head) \\
\hline$r$ & Radius or distance \\
\hline$R N P$ & Rate normalized pressure \\
\hline$R N P^{\prime}$ & Derivative of $R N P$ with respect to $\ln (t)$ \\
\hline$t$ & Time \\
\hline$t_{D y_{e}}$ & Dimensionless time with respect to distance to a boundary \\
\hline$t_{m b}$ & Material balance time \\
\hline$t_{e}$ & Material balance time \\
\hline$t_{\text {elf }}$ & End of linear flow \\
\hline$t_{e, e l f}$ & End of linear flow in material balance time \\
\hline$V_{n}$ & Volume moment integral \\
\hline$V_{p}$ & Pore volume \\
\hline$w(\tau)$ & Derivative of pore volume with respect to $\tau$ \\
\hline$w_{f}$ & Fracture width \\
\hline
\end{tabular}




$\begin{array}{ll}W_{n} & \text { Pressure drop moment integral } \\ x_{f} & \text { Fracture half-length } \\ x_{s} & \text { Fracture spacing } \\ y_{e} & \text { Distance to a boundary }\end{array}$

Greek Symbols

$\alpha$

$\phi$

$\tau$

$\mu$
Reservoir Diffusivity

Porosity

Diffusive Time of Flight

Fluid viscosity 


\section{TABLE OF CONTENTS}

Page

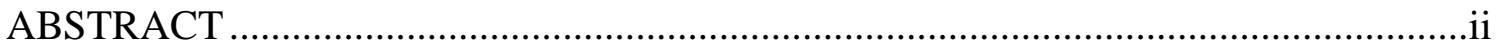

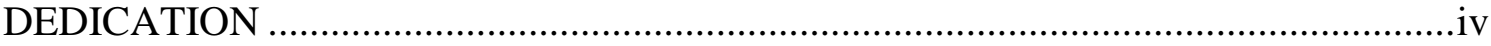

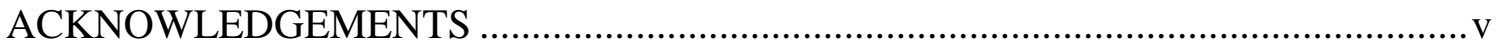

CONTRIBUTORS AND FUNDING SOURCES...........................................................vi

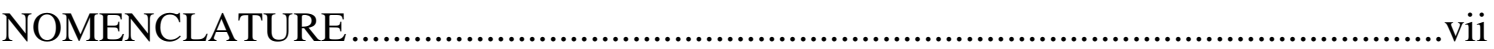

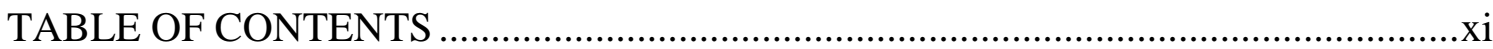

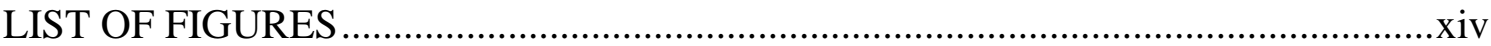

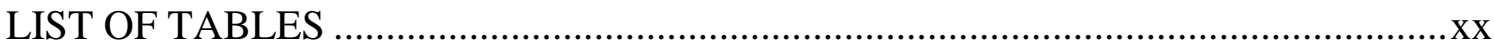

CHAPTER I SHALE OIL \& GAS INTRODUCTION ..................................................

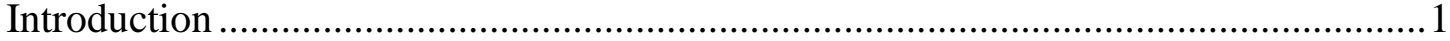

A Brief History of Shale Oil \& Gas ....................................................................

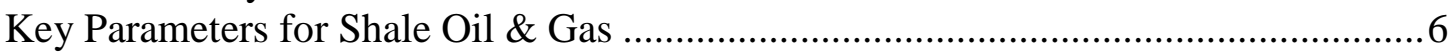

Category 1 - Classical Geologic Factors ………………...........................................

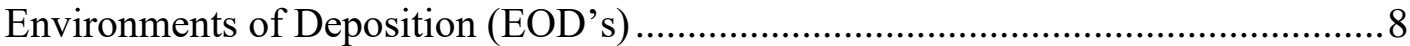

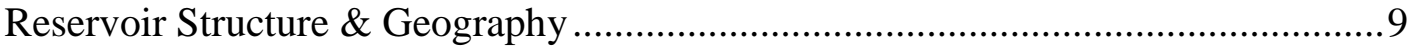

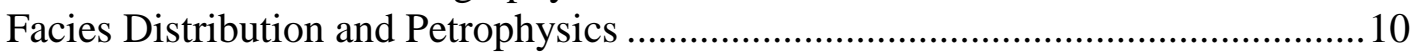

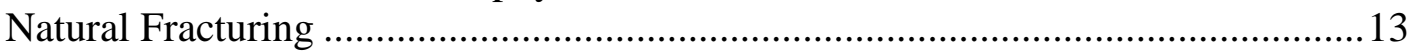

Category 2 - Unconventional Geologic \& Fluid-Flow/Storage Parameters.................14

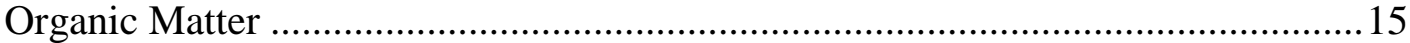

Reservoir Fluids and OM Maturity .................................................................. 18

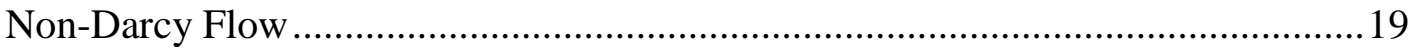

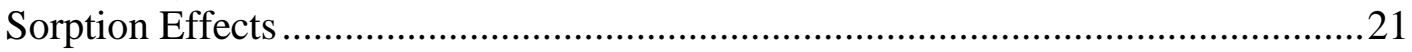

Category 3 - Completion Parameters....................................................................22

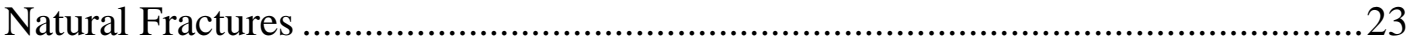

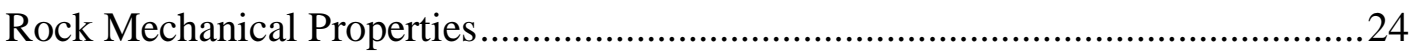

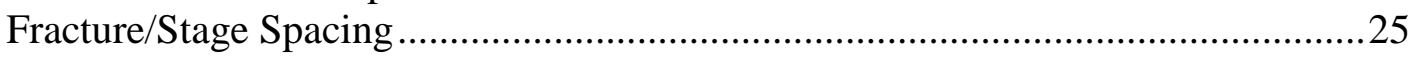

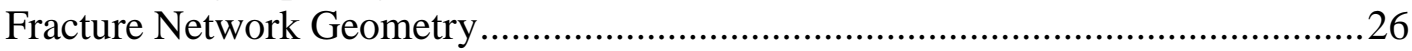

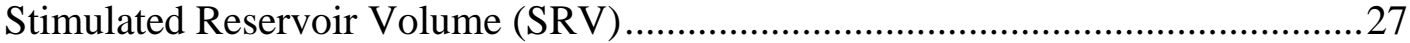

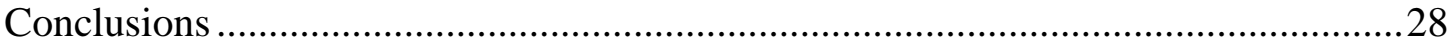




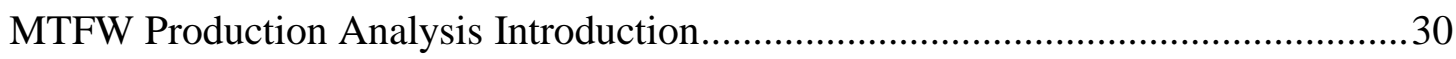

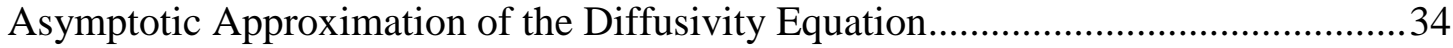

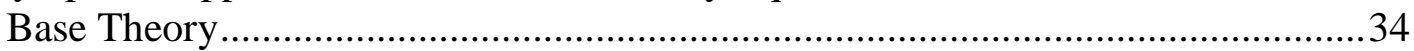

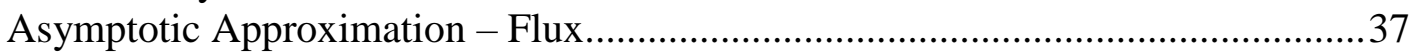

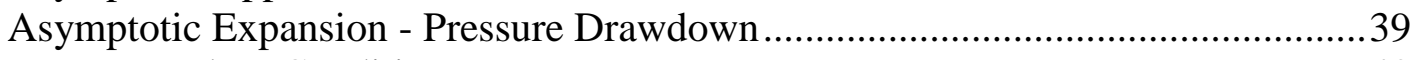

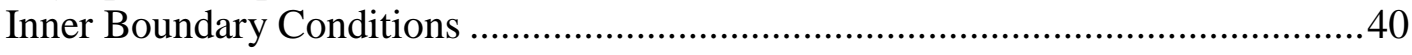

Applying the Asymptotic Approximation to Specific Flow Geometries ....................43

1-Dimensional Solutions - Infinite-Acting Linear Flow (IALF) ...........................43

2-Dimensional Solutions - Infinite Acting "Pillbox" Flow (IAPF).......................48

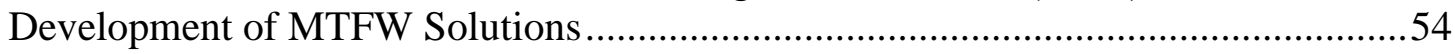

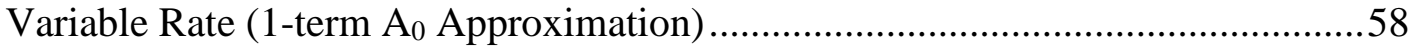

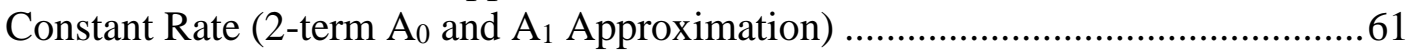

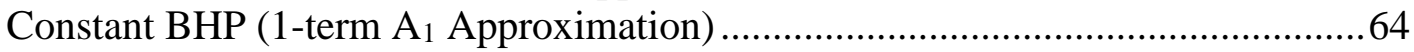

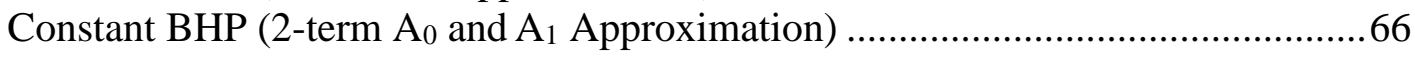

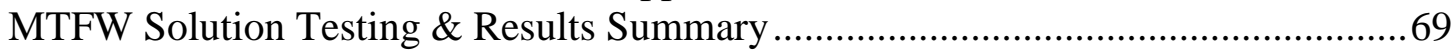

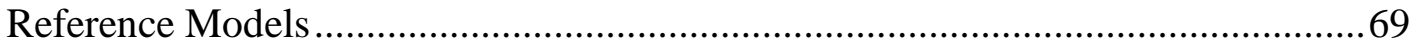

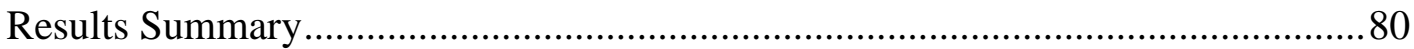

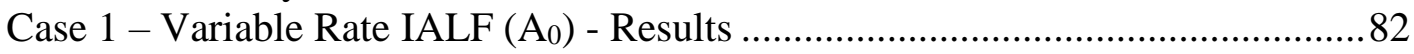

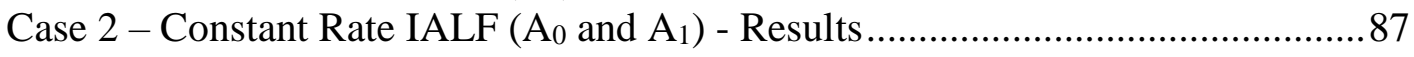

Case 3 - Variable Rate IAPF $\left(\mathrm{A}_{0}\right)$ - Results ................................................... 91

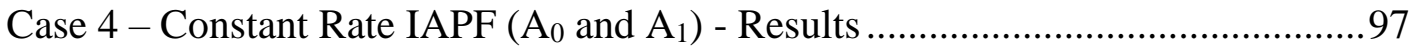

Case 5 - Constant BHP IALF $\left(\mathrm{A}_{1}\right)$ - Results ........................................................ 102

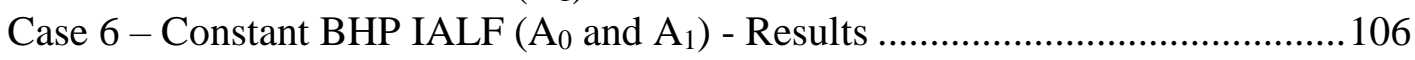

Case 7 - Constant BHP IAPF $\left(\mathrm{A}_{1}\right)$ - Results ................................................. 110

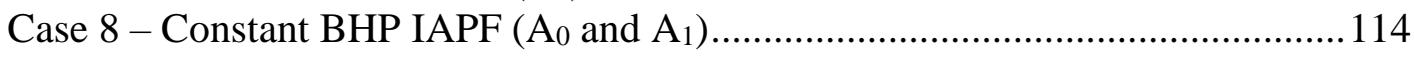

SRV Flow Mechanisms \& Drainage Volumes................................................ 119

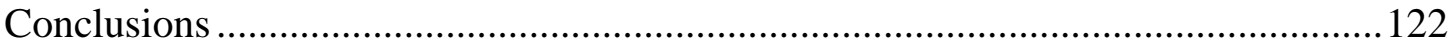

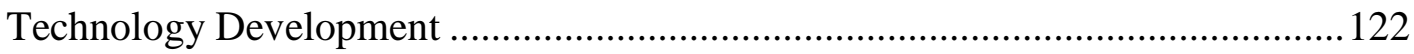

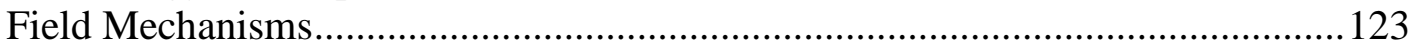

CHAPTER III INVESTIGATION OF THE END OF LINEAR FLOW ..................... 125

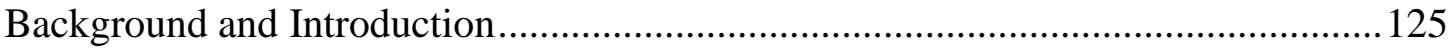

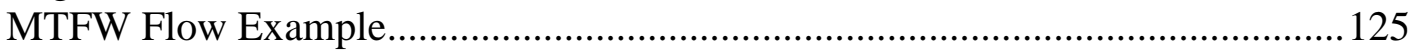

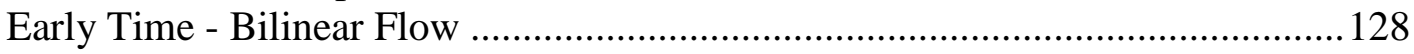

Early/Middle Time - Linear Flow ................................................................. 129

Middle Time - Fracture Interference (Approximate Pseudo Steady State) ........... 129

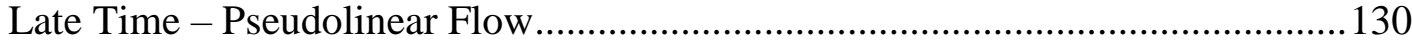

Late Time - Radial Transition................................................................... 130

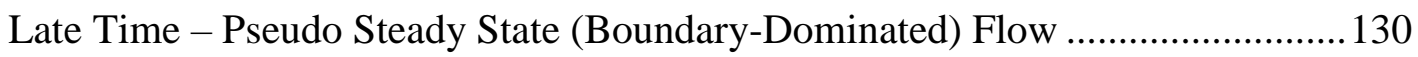

Previous Definitions of the End of Linear Flow ................................................... 135

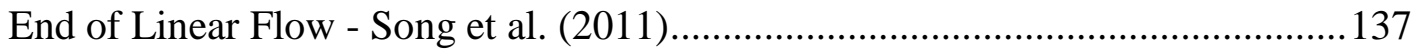


End of Linear Flow - Song and Ehlig-Economides (2011) ................................ 138

End of Linear Flow - Wattenbarger et al. (1998) .................................................139

End of Linear Flow - Lee and Spivey (2013)................................................... 141

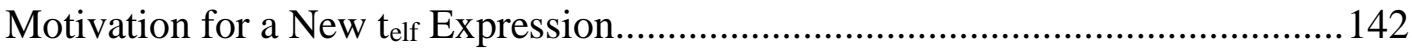

Transient Drainage Volumes........................................................................... 143

End of Linear Flow Based on the Limit of Detectability .......................................149

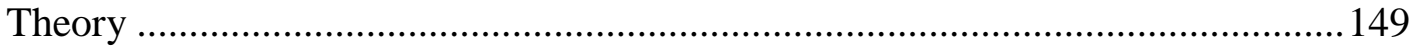

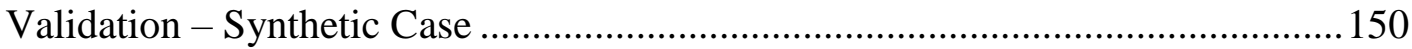

Validation \& Application - Montney Field Case .............................................. 153

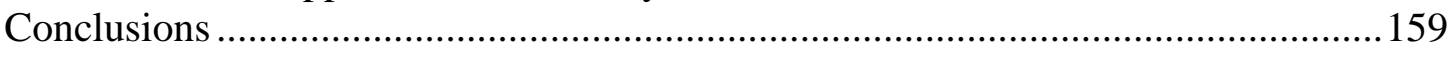

CHAPTER IV SUMMARY AND CONCLUSIONS .......................................... 161

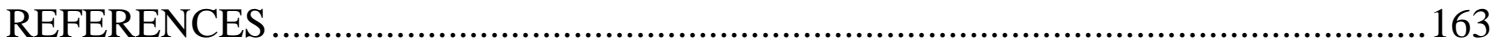




\section{LIST OF FIGURES}

Page

Figure 1 - Unconventional Reservoir Framework - Eagle Ford Reservoir 7

Figure 2 - Eagle Ford Structure - From Hentz (2010) - Republished by permission of the Gulf Coast Association of Geological Societies, whose permission is required for further publication use

Figure 3 - Eagle Ford Shale Extent and Geography - From Hentz (2010) Republished by permission of the Gulf Coast Association of Geological Societies, whose permission is required for further publication use

Figure 4 - Eagle Ford Shale Fluid Production Map - From Yao Tian (2013) -

Republished by permission of the Gulf Coast Association of Geological Societies, whose permission is required for further publication use

Figure 5 - Unconventional Reservoir Framework - This study's focus highlighted in red box

Figure 6 - IALF Diagram (after Malone et al. (2019))

Figure 7 - IALF Coordinates (from Malone et al. (2019)). Copyright 2019, SPE. Reproduced with permission of SPE. Further reproduction prohibited without permission.

Figure 8 - Pillbox Model (after Malone et al. (2019)). Copyright 2019, SPE.

Reproduced with permission of SPE. Further reproduction prohibited without permission.

Figure 9 - Pillbox model distance parameters (from Malone et al. (2019)). Copyright 2019, SPE. Reproduced with permission of SPE. Further reproduction prohibited without permission.

Figure 10 - MTFW System (X-Z Plane View) ....................................................55

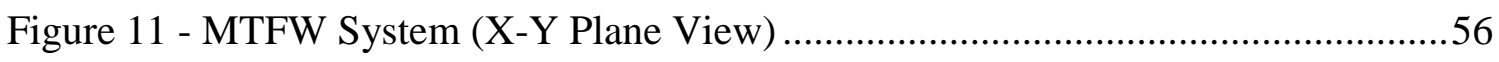

Figure 12 - MTFW System (Y-Z Plane View) .....................................................56

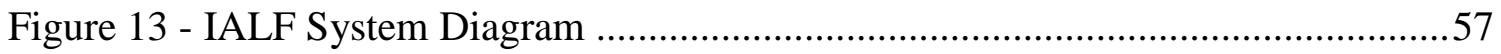


Figure 14 - IAPF System Diagram (after Malone et al. (2019)). Copyright 2019, SPE.

Reproduced with permission of SPE. Further reproduction prohibited

without permission.

Figure 15 - IALF Reference Model Dimensions

Figure 16 - IALF Reference Model Screenshot (Pressure Property Displayed) 70

Figure 17 - IAPF Reference Model Dimensions (from Malone et al. (2019)).

Copyright 2019, SPE. Reproduced with permission of SPE. Further reproduction prohibited without permission. 72

Figure 18 - IAPF Reference Model Screenshot (Pressure Property Displayed) 72

Figure 19 - IALF Constant-Rate Simulation Diagnostic Plot 73

Figure 20 - IAPF Constant-Rate Simulation Diagnostic Plot (after Malone et al. (2019)). Copyright 2019, SPE. Reproduced with permission of SPE. Further reproduction prohibited without permission.

Figure 21 - IAPF Constant-Rate Simulation - Elliptical Flow Dip Signature (zoomed in view) (after Malone et al. (2019)). Copyright 2019, SPE. Reproduced with permission of SPE. Further reproduction prohibited without permission.

Figure 22 - IALF Constant-BHP Simulation Diagnostic Plot.

Figure 23 - IALF Constant-BHP Simulation Diagnostic Plot $\left(\mathrm{t}_{\mathrm{mb}}\right)$

Figure 24 - IAPF Constant BHP Simulation Diagnostic Plot (after Malone et al. (2019)). Copyright 2019, SPE. Reproduced with permission of SPE. Further reproduction prohibited without permission.

Figure 25 - IAPF Constant BHP Simulation Diagnostic Plot $\left(\mathrm{t}_{\mathrm{mb}}\right) \ldots \ldots \ldots \ldots \ldots \ldots \ldots \ldots \ldots \ldots \ldots \ldots . . .79$

Figure 26 - IAPF Constant BHP Simulation Diagnostic Plot $\left(\mathrm{t}_{\mathrm{mb}}\right)-\ldots \ldots \ldots \ldots \ldots \ldots \ldots \ldots \ldots \ldots \ldots . . .80$

Figure 27- Test Model Diagram - Fracture Numbering ................................................82

Figure 28 - Case 1 Diagnostic Plot Comparison with Reference Model...........................84

Figure 29 - Case 1 - Individual Fracture Flowrates ………..............................................8

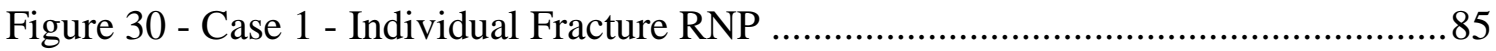

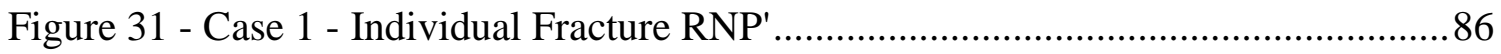




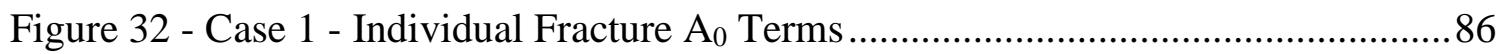

Figure 33 - Case 2 Diagnostic Plot Comparison with Reference Model ............................88

Figure 34 - Case 2 - Individual Fractures Flowrates .........................................................8

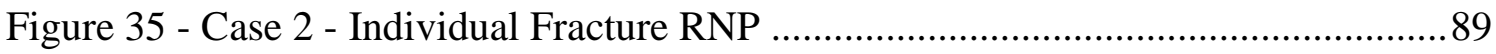

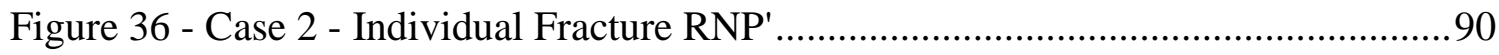

Figure 37 - Case 2 - Individual Fracture A 0 Terms ......................................................90

Figure 38 - Case 2 - Individual Fracture $A_{1}$ Terms .........................................................

Figure 39 - Case 3 Diagnostic Plot Comparison with Reference Model (after Malone et al. (2019)). Copyright 2019, SPE. Reproduced with permission of SPE. Further reproduction prohibited without permission.......................................92

Figure 40 - Case 3 Diagnostic Plot - Elliptical "Dip" Signature (after Malone et al. (2019)). Copyright 2019, SPE. Reproduced with permission of SPE. Further reproduction prohibited without permission.......................................................93

Figure 41 - Case 3 - Individual Fractures Flowrate (after Malone et al. (2019)). Copyright 2019, SPE. Reproduced with permission of SPE. Further reproduction prohibited without permission.

Figure 42 - Case 3 - Individual Fracture RNP (after Malone et al. (2019)). Copyright 2019, SPE. Reproduced with permission of SPE. Further reproduction prohibited without permission.

Figure 43 - Case 3 - Individual Fracture RNP' (after Malone et al. (2019)). Copyright 2019, SPE. Reproduced with permission of SPE. Further reproduction prohibited without permission.

Figure 44 - Case 3 - Individual Fracture $A_{0}$ Terms (after Malone et al. (2019)). Copyright 2019, SPE. Reproduced with permission of SPE. Further reproduction prohibited without permission .96

Figure 45 - Case 4 Diagnostic Plot Comparison with Reference Model (after .98

Figure 46 - Case 4 - Elliptical Flow Dip Signature (after Malone et al. (2019)). Copyright 2019, SPE. Reproduced with permission of SPE. Further reproduction prohibited without permission.

Figure 47 - Case 4 - Individual Fractures Flowrates .99 
Figure 48 - Case 4 - Individual Fracture RNP ………………................................... 100

Figure 49 - Case 4 - Individual Fracture RNP' .................................................................100

Figure 50 - Case 4 - Individual Fracture A $A_{0}$ Terms ......................................................101

Figure 51 - Case 4 - Individual Fracture $A_{1}$ Term .....................................................101

Figure 52 - Case 5 Diagnostic Plot Comparison with Reference Mode …………….....103

Figure 53 - Case 5 - Individual Fractures Flowrates ....................................................... 104

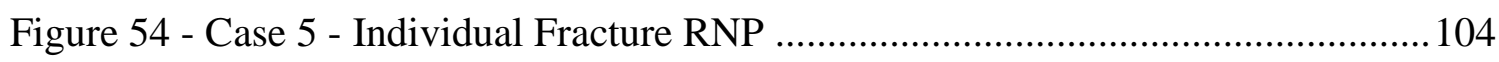

Figure 55 - Case 5 - Individual Fracture RNP' ........................................................... 105

Figure 56 - Case 5 - Individual Fracture $A_{1}$ Terms ....................................................... 105

Figure 57 - Case 6 Diagnostic Plot Comparison with Reference Model ........................107

Figure 58 - Case 6 - Individual Fractures Flowrates ........................................................107

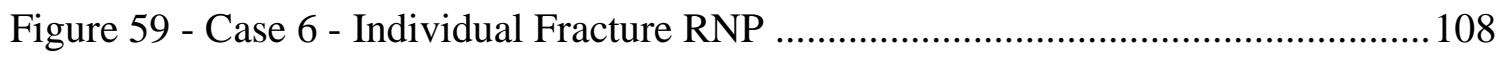

Figure 60 - Case 6 - Individual Fracture RNP' ..............................................................108

Figure 61 - Case 6 - Individual Fracture A $A_{0}$ Terms .......................................................109

Figure 62 - Case 6 - Individual Fracture $A_{1}$ Terms ........................................................109

Figure 63 - Case 7 Diagnostic Plot Comparison with Reference Model .........................111

Figure 64 - Case 7 - Individual Fractures Flowrates ........................................................111

Figure 65 - Case 7 - Individual Fracture RNP …………………………………….....112

Figure 66 - Case 7 - Individual Fracture RNP' ...............................................................112

Figure 67 - Case 7 - Individual Fracture $A_{1}$ Terms ........................................................113

Figure 68 - Case 8 Diagnostic Plot Comparison with Reference Model (after Malone et al. (2019)). Copyright 2019, SPE. Reproduced with permission of SPE. Further reproduction prohibited without permission. .......................................115

Figure 69 - Case 8 - Individual Fractures Flowrates ....................................................116

Figure 70 - Case 8 - Individual Fracture RNP …………………………….............116 
Figure 71 - Case 8 - Individual Fracture RNP'.

Figure 72 - Case 8 - Individual Fracture $\mathrm{A}_{0}$ Terms

Figure 73 - Case 8 - Individual Fracture $A_{1}$ Terms

Figure 74 - Individual fracture drainage volumes - Case 1

Figure 75 - Individual fracture drainage volumes - Case 3 (after Malone et al. (2019)). Copyright 2019, SPE. Reproduced with permission of SPE. Further reproduction prohibited without permission.

Figure 76 - Example Model Diagram (from Malone et al. (2019)). Copyright 2019, SPE. Reproduced with permission of SPE. Further reproduction prohibited without permission.

Figure 77 - Example MTFW Model Diagnostic Plot (after Malone et al. (2019))

Copyright 2019, SPE. Reproduced with permission of SPE. Further reproduction prohibited without permission.

Figure 78 - Example MTFW Model Diagnostic Plot (Flow Regimes Labelled) (after Malone et al. (2019)). Copyright 2019, SPE. Reproduced with permission of SPE. Further reproduction prohibited without permission.

Figure 79 - Early Time - Bilinear Flow (from Malone et al. (2019)). Copyright 2019, SPE. Reproduced with permission of SPE. Further reproduction prohibited without permission.

Figure 80 - Early/Middle Time - Linear Flow (from Malone et al. (2019)). Copyright 2019, SPE. Reproduced with permission of SPE. Further reproduction prohibited without permission.

Figure 81 - Middle Time - Fracture Interference \& Apparent Pseudo Steady State (from Malone et al. (2019)). Copyright 2019, SPE. Reproduced with permission of SPE. Further reproduction prohibited without permission.

Figure 82 - Late Time - Compound Linear Flow

Figure 83 - Late Time - Radial Flow

Figure 84 - Late Time - Transition to Pseudo Steady State.

Figure 85 - Late Time - True Pseudo Steady State

Figure 86 - End of Linear Flow 136 
Figure 87 - End of Linear Flow Equations (from Malone et al. (2019)). Copyright 2019, SPE. Reproduced with permission of SPE. Further reproduction prohibited without permission.

Figure 88 - Wattenbarger Type Curve Geometry (Modeled after Wattenbarger et al.

Figure 89 - Wattenbarger Type Curve (Based on Wattenbarger et al. (1998))

Figure 90 - Hydraulic Fracture Drainage Areas

Figure 91 - Pressure propagation representation (constant rate)

Figure 92 - Pressure propagation representation (constant BHP)

Figure 93 - Diffusion kernel spatial profile (from Malone et al. (2019)). Copyright 2019, SPE. Reproduced with permission of SPE. Further reproduction prohibited without permission.

Figure 94 - End of Linear Flow - Limit of Detectability (Constant Rate Case).

Figure 95 - End of Linear Flow - Limit of Detectability (Constant BHP Case) ............. 152

Figure 96 - End of Linear Flow - Limit of Detectability (Constant BHP Case) ............ 153

Figure 97 - End of Linear Flow conversion from real time to material balance time ... 153

Figure 98 - Montney Field Case Production Data (From Wang et al. (2018)) 155

Figure 99 - Montney field case optimized drainage volume (left) and w(t) (right) curves (From Wang et al. (2018)).............................................................. 156

Figure 100 - Montney field case diagnostic plot (left) and specialized plot (right)

(From Wang et al. (2018)) 156

Figure 101 - Time conversion plot (after Malone et al. (2019)). Copyright 2019, SPE. Reproduced with permission of SPE. Further reproduction prohibited without permission. 


\section{LIST OF TABLES}

Page

Table 1 - Reference Model Reservoir Properties (from Malone et al. (2019)). Copyright 2019, SPE. Reproduced with permission of SPE. Further reproduction prohibited without permission

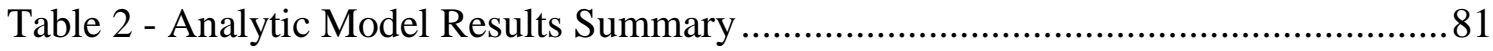

Table 3 - Example Model Properties.................................................................... 127

Table 4 - Diffusion Kernel and Boltzmann Variable values for asymptotic approximation solution (from Malone et al. (2019)). Copyright 2019, SPE. Reproduced with permission of SPE. Further reproduction prohibited without permission.

Table 5 - Synthetic Model Properties (from Malone et al. (2019)). Copyright 2019, SPE. Reproduced with permission of SPE. Further reproduction prohibited without permission.

Table 6 - Montney Field Case Well \& Reservoir Properties (From Wang et al. (2018))

Table 7 - Permeability Estimation Comparison (from Malone et al. (2019)). Copyright 2019, SPE. Reproduced with permission of SPE. Further reproduction prohibited without permission. 


\section{CHAPTER I}

\section{SHALE OIL \& GAS INTRODUCTION}

\section{Introduction}

Shale oil and shale gas reservoirs have dominated a growing share of the oil and gas industry's attention for decades. With immense resource potential and technical complexity, these reservoirs present us with a great opportunity for natural resource development and continued scientific discovery. Over the years since Mitchell Energy Development Corp. (MEDC) first successfully pioneered production from the Barnett Shale, the production of oil and gas from ultra-tight reservoirs has exploded on a global scale (Jarvie 2012a). With recent technological advancement, more shale resources have become economic, and more countries have been able to participate in the "shale revolution" as our understanding of production from ultra-tight rocks has improved. This is reflected by growth in estimated resource volumes. Rogner (1997) estimated total worldwide shale gas volume to be $16 * 10^{15}$ cubic feet. 14 years later, EIA (2011) estimated it to be $22 * 10^{15}$ cubic feet, and updated this number to $31 * 10^{15}$ in 2013 (EIA 2013). This prolific rise in resources comes from both increases on local scales, and from the inclusion of more nations in each report.

One of the greatest commonalities between shale plays remains the necessary drilling of horizontal wells completed with multi-stage hydraulic fracturing stimulation treatments - otherwise known as multiple transverse fracture wells (MTFW's). This drilling and completion strategy is the primary reason that production from ultra-tight oil and gas resources became economic in the first place (Jarvie 2012a). The production of 
oil and gas from shales through MTFW's presents interesting challenges that come about as a result of complexity in both rock properties and geometric parameters. Successfully developing shale plays depends upon evaluators' abilities to identify and interpret both the important geologic/reservoir parameters and the geometric completion-related parameters that control the fluid flow in MTFW's (Cipolla et al. 2011).

Previous work from our research group (Agarwal 2011) discussed the importance of prior assumptions made about reservoir parameters in the context of a history-matching exercise for an offshore turbidite reservoir. Our goal in this new study is to approach shale reservoir evaluation through the same lens, focusing on both the geology and completion-related complexities present in MTFW's. Through rigorous literature review we identify the key geologic and engineering parameters for unconventional shale reservoirs and contextualized those parameters as they apply to a single shale play - the south Texas Eagle Ford play. We present this review and list in this chapter. From this list we approach a subset of problems - those pertaining to the nature of fluid flow in MTFW's and the onset of flow interference between neighboring hydraulic fractures - and develop analytic methods for the interpretation of production data as it pertains to MTFW's.

Using the asymptotic approximation of the diffusivity equation (King et al. 2016) in conjunction with the principles of superposition in space and linearity, we are able to accurately model the pressure transient behavior of a MTFW while taking the effects of fracture interference into account. We developed formulations for both constant-rate and constant-BHP inner boundary conditions for both linear and "pillbox" flow models. 
These models are able to compute the rate-normalized pressure drop (RNP) and its derivative with respect to the natural logarithm of time (RNP') on a by-fracture or whole-well basis for a single MTFW. We validate this model by comparison with a commercial simulator. As it stands, our constant-rate formulations either exactly or approximately match simulator results, and our constant-BHP formulations see varying but lesser degrees of success. We discuss the development of these formulations in Chapter II.

Production from shale reservoirs is ultimately difficult because it is the production of fluids from extremely low-permeability rock (Blasingame 2008). This low permeability leads to transient flow periods that exceed those observed in conventional reservoirs even by orders of magnitude. For a MTFW, this early transient flow commonly takes the form of either linear or bilinear flow (Clarkson 2013; Song and Ehlig-Economides 2011) and eventually transitions to an apparent pseudo-steady state flow regime as hydraulic fractures begin to interact. We refer to the time at which this interaction begins as the "end of linear flow", or telf.

We find that the current literature fails to fully understand the end of linear flow. Numerous definitions for the end of linear flow exist in the literature (Ehlig-Economides 1992; Lee et al. 2003; Song et al. 2011; Song and Ehlig-Economides 2011; Wattenbarger et al. 1998). None are truly in agreement - neither regarding the physical nature of the end of linear flow nor the mathematical definition. Authors generally agree that the end of linear flow should be defined using either the depth of investigation or some analogous principle but find no consistency regarding the specific equation. We propose 
with this study that the "limit of detectability" (LOD) concept provides an ample method for defining the end of linear flow and guiding its detection in the field when analyzing production data. We substantiate this claim with numerical simulation results from a commercial simulator, detailed diagnostic data from our aforementioned analytic model, and with a field example from the Montney shale play in Canada.

\section{A Brief History of Shale Oil \& Gas}

The ability to exploit hydrocarbons contained in rocks with nano-darcy permeability is not an extremely new concept. In 1821 gas was produced from the Devonian Dunkirk Shale in Appalachia (Sondergeld et al. 2010b). In the early-to-mid 1900's, the Columbia Gas System Service Corp developed Devonian shales in Appalachia (Sondergeld et al. 2010b). The "shale boom" began later on when Mitchell Energy \& Development Corporation (MEDC) became involved with the Barnett Shale (Hinton 2012).

MEDC drilled their first shale gas well, C.W. Slay No. 1, in 1981 (Hinton 2012). Spurred on by potential new revenue under the Natural Gas Policy Act, George P. Mitchell sought to revitalize his company's already-developed acreage near Fort Worth, Texas with new shale production (Hinton 2012). The C.W. Slay No. 1 was not economic, and MEDC staff struggled to unlock the secret to shale gas exploitation until their major breakthrough in 1997 (Hinton 2012). By using slickwater in lieu of the more common viscous hydraulic fracturing fluids, MEDC was able to both cut costs and 
improve well performance, transforming the Barnett Shale from an unproducible source rock to a massive potential reservoir rock (Hinton 2012).

The Barnett Shale boom soon followed, and MEDC was later purchased by Devon Energy in 2002 (Jarvie 2012a). Devon continued to pursue production in the Barnett, and Southwestern Energy soon announced success in the Fayetteville Shale of Arkansas (Jarvie 2012a). MEDC's expertise propagated through the industry, and we saw the "shale revolution" that led to where the industry is today (Jarvie 2012a) with recoverable shale oil and gas resources in over 137 formations and 42 countries worldwide (EIA 2013). 


\section{Key Parameters for Shale Oil \& Gas}

In the usual, or "conventional", context, shales are seen as seals, traps, and source rocks - anything but a reservoir rock (Clarkson et al. 2016). This paradigm persisted until improvements in hydraulic fracturing technology enabled economic production from shales (Clarkson et al. 2016). In a general sense, this is the greatest similarity between shale reservoirs - economic production from any ultra-tight shale reservoir will require large-scale stimulation through hydraulic fracturing or some yet undiscovered technology.

Another great similarity between shales is their variance. No two shales are the same. They can produce oil, gas, condensate, or a diverse mixture of fluids, varying with both spatial distribution and kerogen maturity (Jarvie 2012a, 2012b). They originate from both marine and lacustrine environments (Zou 2017). Mineralogically, shales can form as siliclastic rocks, calcareous rocks, or a mixture of the two (Jarvie 2012a, 2012b). Though many cite shales as clay-heavy, their clay content can vary substantially from basin to basin, and even show both small-scale and large-scale heterogeneities within a single formation (Bohacs et al. 2013; Passey et al. 2010).

This introductory chapter will address the various generalized challenges we face in shale reservoir evaluation through a review of recent literature in the fields of unconventional reservoir geology, petrophysics, and reservoir engineering. Since the field of unconventional resources is so broad, we present generalized challenges, and contextualize them as they apply to a single reservoir - the Eagle Ford Shale. We strive to maintain this list of challenges as a foundation for future studies in unconventional 
resources. In Chapters 2 and 3, we present our work intended to address just a few of the challenges we list.

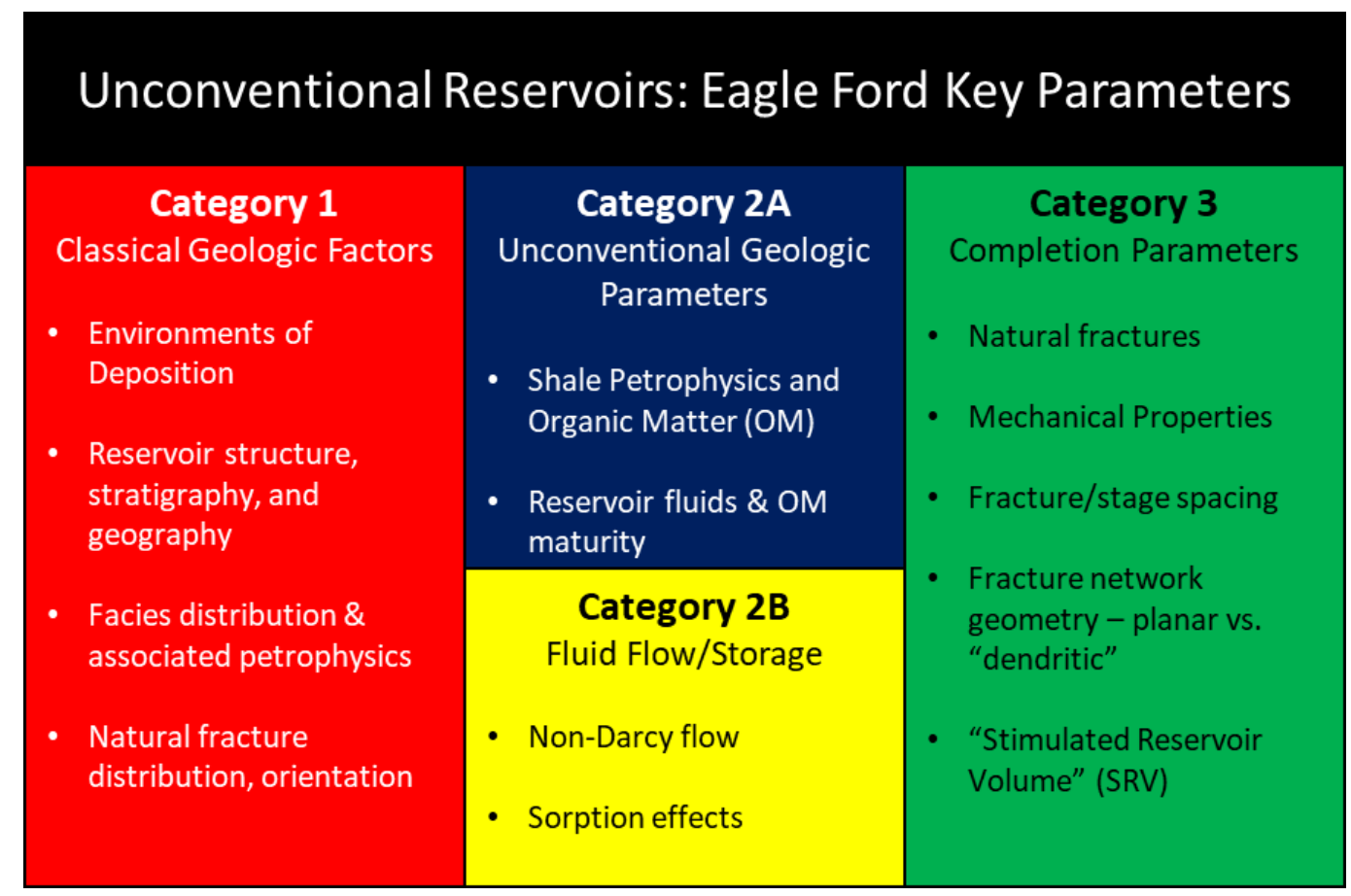

Figure 1 - Unconventional Reservoir Framework - Eagle Ford Reservoir

\section{Category 1 - Classical Geologic Factors}

Though unconventional reservoirs present their own unique challenges to evaluators, classical tools provided by geology, petrophysics, and reservoir engineering still apply when attempting to produce from shales (Bohacs et al. 2013). In Category 1, we briefly address the more "conventional" parameters that apply to unconventional reservoirs and to the Eagle Ford shale. 


\section{Environments of Deposition (EOD's)}

Authors tend to agree that shales are defined by a very small grain size. Exact definitions of "shale" via grain size vary, but many authors will argue that shales should really be referred to as producible mudstones, and have grain sizes less than approximately 65 micrometers (Bohacs et al. 2013; Loucks et al. 2009; Sondergeld et al. 2010a; Sondergeld et al. 2010b). This categorization lends itself to a simple and broad description of shale environments of deposition (EOD's) - hydrocarbon-bearing shales are formed in low-energy environments where these small particles can accumulate and where the prerequisites for organic matter accumulation and maturation are met (Bohacs et al. 2013; Passey et al. 2010). These can be terrestrial or marine settings where surface water plankton productivity is high, water is deep, oxygen content is poor, and the ratio of organic matter production to sediment influx is balanced (Passey et al. 2010; Zou 2017). Typical terrestrial EOD’s are deep lakes (Zou 2017) and typical marine environments include constructional shelf margins, platforms and ramps, and continental slope basins (Passey et al. 2010).

The Eagle Ford shale already breaks from the unconventional mold in that it is not entirely composed of shale intervals. The Cretaceous Eagle Ford shale is well known as the source rock for the overlaying Austin Chalk formation (Donovan and Staerker 2010) and certainly includes organic-rich mudstone layers, but is actually what Jarvie (2012b) refers to as a "hybrid system". A hybrid system is one composed of organic-rich mudstone layers interbedded with non-shale intervals (Jarvie 2012b). In the Eagle Ford, these non-shales are carbonate beds that crop up with varying frequency, dependent 
upon their location in the Eagle Ford basin and depth within the Eagle Ford formation itself (Hentz 2010; Lock et al. 2010). Eagle Ford black shales were most likely formed as seabed intervals when ocean levels were higher during transgressive cycles (Donovan and Staerker 2010).

\section{Reservoir Structure \& Geography}

Broadly defining reservoir structure for an entire subset of reservoirs, e.g. shales, is difficult because no two reservoirs are the same. This being said, shales are often referred to as "resource plays" (Russell and Freeborn 2012) for a reason. Shales are typically structurally uninteresting on a small scale and show a large-scale shape that simply reflects the geography of their original deposition and subsequent burial and deformation.

The Eagle Ford shale's structure reflects the locations of various basins that existed during its original deposition. It lies mostly in Texas, and is well known for sitting above the Buda Limestone and beneath the Austin Chalk (Donovan and Staerker 2010; Hentz 2010; Lock et al. 2010). Within Texas, the Eagle Ford extends from the southwest border with Mexico up to the East Texas Basin where the Sabine uplift sits (Hentz 2010). The Eagle Ford is often broken into two sections, the Upper Eagle Ford (UEF) and Lower Eagle Ford (LEF), which evaluators can identify easily on wireline logs through a higher gamma ray signature through the LEF than what is seen in the UEF, primarily due to higher organic content in the LEF (Hentz 2010). The LEF is present through the entirety of the Eagle Ford and has a much greater black shale content 
and higher total organic carbon (TOC) (Donovan and Staerker 2010; Hentz 2010; Lock et al. 2010; Tian et al. 2013). The UEF is typically less productive, has less organic matter, and has higher carbonate content (Donovan and Staerker 2010; Hentz 2010; Lock et al. 2010; Tian et al. 2013).

The Eagle Ford is often broken up into 3 major geographic localities - the Maverick Basin, the San Marcos Arch, and the East Texas Basin (Hentz 2010) (Figure 3). The Maverick Basin holds the deepest Eagle Ford accumulations and represents the thickest portion of the Eagle Ford shale, presenting both UEF and LEF intervals (Hentz 2010). Moving northeast from the Maverick Basin, the Eagle Ford begins to thin as it approaches the San Marcos Arch (Hentz 2010). The UEF eventually pinches out in Atascosa county, and appears again only briefly in one instance, mostly in Gonzales County (Hentz 2010). As we approach the East Texas Basin further northwest, the Buda Limestone and Austin Chalk grow further apart, but this growth does not necessarily make the Eagle Ford thicker (Lock et al. 2010). Other groups - namely the Pepper Shale and the Sandy Woodbine Group, appear in intervals beneath the LEF, separating it from the underlying Buda Limestone (Figure 2) (Lock et al. 2010).

\section{Facies Distribution and Petrophysics}

Since we intend to discuss "classical" parameters in this section, we will avoid discussion of unconventional petrophysics topics like organic matter, which we will discuss later. Shale reservoirs can present siliclastic or calcareous lithologies, with varying levels of clay content (Bohacs et al. 2013; Passey et al. 2010). The oft-cited high 
gamma ray response of shales can come about as a result clay content, but also happens to be a signature of high total organic carbon (TOC) (Bohacs et al. 2013; Passey et al. 2010). Shales are well-known for extreme anisotropy, which is a result of the large-scale alignment of mineral grains and clay platelets by compaction processes, that typically results in much higher bed-parallel (horizontal) permeability relative to bedperpendicular (vertical) permeability (Sondergeld et al. 2010a). Perhaps the most important petrophysical property of shales is their permeability, which is extremely low (Blasingame 2008). Hard limits on shale permeability don't necessarily exist, but soft limits are forced upon us by nature. The combination of fluid resistance to flow (viscosity) and a rock's tendency to transmit fluids (permeability) will dictate what shales can ultimately produce economically. Gas shales are documented to have produced in as low as the nanodarcy range (less than 1,000 nanodarcies), and shale oil is typically producible in the high nano-darcy range (not much less than 1,000 nanodarcies) to the microdarcy range (greater than 1,000 nanodarcies) (Bohacs et al. 2013). The extremely low permeability of shale correlates to its very small grain size and resulting tiny pore throats (Nelson 2009). Porosity values can be very small (as low as 1-2\% in Sondergeld et al. (2010a)) or surprisingly high (Bohacs et al. (2013) reports 15\%. 


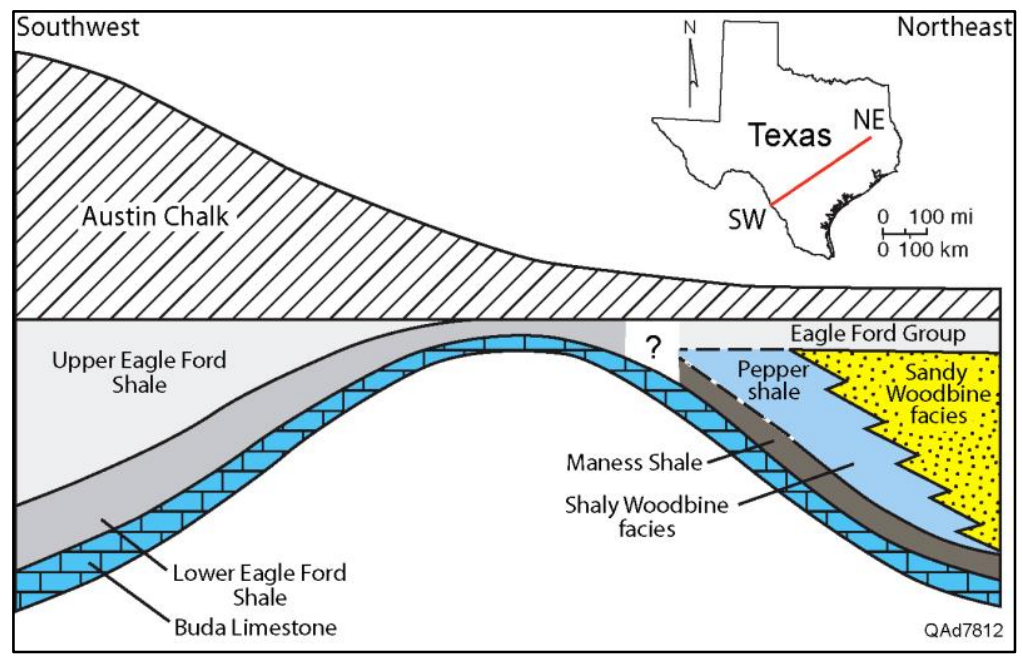

Figure 2 - Eagle Ford Structure - From Hentz (2010) - Republished by permission of the Gulf Coast Association of Geological Societies, whose permission is required for further publication use

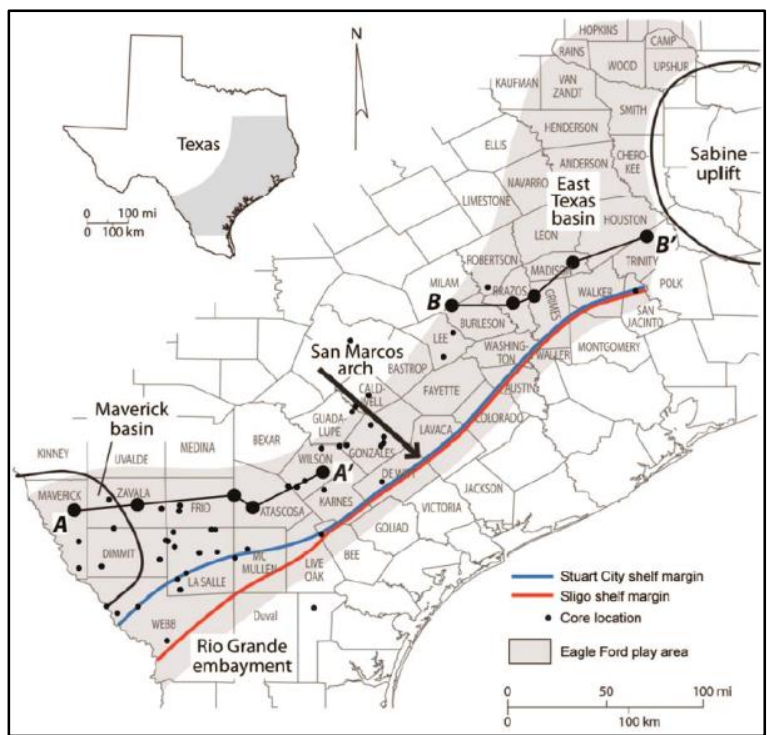

Figure 3 - Eagle Ford Shale Extent and Geography - From Hentz (2010) Republished by permission of the Gulf Coast Association of Geological Societies, whose permission is required for further publication use 
in some samples) but vary heavily between basins, and even between core samples within single basins. As it is a "hybrid" reservoir, the Eagle Ford shale exhibits a wide variety of facies and associated petrophysical properties. Dawson (2000) documents a total of six major facies groups observed in Eagle Ford outcrop and subsurface core rock samples. They report the presence of pyritic shale (facies 1), phosphatic shale (facies 2), bentonitic shale (facies 3), fossiliferous shale (facies 4), silty shale (facies 5), and bituminous claystone (facies 6) in studied core samples (Dawson 2000). Donovan and Staerker (2010) reports a series of calcareous mudstones with interbedded wackestones and packstones observed in an Eagle Ford outcrop interval. Eagle Ford porosities typically sit between $3 \%$ and $9 \%$, with permeabilities less than $0.1 \mathrm{mD}$ (Zou 2017).

\section{Natural Fracturing}

Shales are known for producing at rates much higher than expected for nanodarcy rock, and natural fractures provide a likely source for this excess production (Gale et al. 2014). Natural fractures occur frequently in shales, particularly those with low clay content and high brittleness (Gale et al. 2014). Natural fractures in shales are highly prone to sealing via re-compaction, and those that stay open often see the precipitation of mineral fill over time, which can either hurt or help production (Gale et al. 2014). Shale fracture systems tend to form at high angle to the bedding plane of their associated intervals but can also form parallel to bedding (Gale et al. 2014). The role of natural fractures in production from shales remains somewhat nebulous because it can be very difficult to evaluate the effects of natural fractures on production separately from 
the effects of hydraulic fractures, especially when considering the complex interactions observed between hydraulic and natural fractures (Gale et al. 2014). These interactions will be discussed later.

Ferrill et al. (2014) suggests that the Eagle Ford experienced numerous deformation events that should lead to natural fracturing. The Eagle Ford has natural fractures associated with salt growth, tertiary extension, the Balcones fault system, and most importantly, the Laramide deformation during the late Cretaceous period (Ferrill et al. 2014). An outcrop study of the Eagle Ford revealed two main fracture sets - one primary set with a southwest-to-northeast strike that likely formed before the secondary southeast-to-northwest striking set that tends to abut against the primary set (Ferrill et al. 2014). These fractures mostly formed perpendicular to the bedding plane, and showed consistency in orientation (Ferrill et al. 2014). Eagle Ford reservoir models have been shown to benefit from the inclusion of natural fractures in numerical simulations (Cipolla et al. 2011; Li et al. 2018; Sun et al. 2016), however, it remains unclear whether this is because natural fractures affect fluid flow substantially on their own, or because of their interactions with induced hydraulic fractures.

\section{Category 2 - Unconventional Geologic \& Fluid-Flow/Storage Parameters}

Though unconventional reservoirs are effectively producible mudrocks, they possess one quality that starkly separates them from conventional reservoirs - the presence of organic solids in the rock matrix of source rock intervals. To compound upon the complexity seen in their evaluation, the nature of flow in unconventional 
reservoirs deviates from classical expectations due to the presence of nano-pores in lieu of the micro-pores commonly seen in conventional reservoirs. In this section, we discuss these and other geologic properties which are truly unique to unconventional reservoirs, and what effect they have on engineering evaluations.

\section{Organic Matter}

Many of the complications seen in quantifying shale reservoir properties stem from the duality of the shale (or source rock) matrix. Shale reservoirs are effectively producible source rock that contain residual un-released hydrocarbons that were originally produced by the thermal maturation of in-situ kerogen (Cipolla et al. 2011; Passey et al. 2010). This in-situ kerogen should be considered a secondary matrix element, and any evaluation of a shale resource needs to consider its effect on fluid storage capacity, fluid flow, geomechanics, and field measurements (Clarkson et al. 2016; Loucks et al. 2009; Sondergeld et al. 2010a; Sondergeld et al. 2010b; Wang and Reed 2009).

The presence of organic matter $(\mathrm{OM})$ in unconventional reservoir rocks introduces entirely new dimensions to their petrophysical evaluation. The porosity of OM usually differs from that of the coexisting inorganic matrix components, enough so that even up to $50 \%$ of a shale's total porosity may be contained within the OM (Passey et al. 2010; Sondergeld et al. 2010a). Scanning electron microscope (SEM) studies revealed that OM typically hosts an internal pore network of its own (Loucks et al. 2009; Sondergeld et al. 2010a; Wang and Reed 2009) and Loucks et al. (2009) suggested that 
the porosity of this internal pore network depends upon the OM's thermal maturity and can be related to vitrinite reflectance $\left(\mathrm{R}_{\mathrm{o}}\right)$ measured in a laboratory setting. Quantification of OM content can help evaluators determine the "sweet spots" along a horizontal wellbore (Cipolla et al. 2011; Passey et al. 2010)

OM complicates permeability measurements as well. OM interacts with oil and gas in unique ways that inorganic matrix components do not, and this can influence measurements made in the laboratory setting (Cui et al. 2009). OM is not the sole culprit however - the anisotropy present in shales, and the nano-scale nature of flow in shale samples are also responsible (Sondergeld et al. 2010a; Sondergeld et al. 2010b). Sondergeld et al. (2010b) and Passey et al. (2010) document even order-of-magnitude discrepancies between permeabilities measured by different labs on identical rock samples of organic-rich shales. Most core lab permeability measurements are based on Darcy's Law (Sondergeld et al. 2010b), which many authors argue does not apply to nano-scale flow (Akkutlu and Fathi 2012; Javadpour 2009), and use core flooding methods that suffer from inherent inadequacies when measuring flow through ultra-tight porous media (Sondergeld et al. 2010b). Sondergeld et al. (2010b) argues that these issues beg for the standardization of core lab tests industry-wide, and that the only trusted permeability measurements should be those made using the pulse decay and pressure decay methodologies.

Another unique property of $\mathrm{OM}$ is its density. OM densities typically vary from 1.1-1.4 g/cc (Passey et al. 2010), which is substantially lower than the densities of typical matrix elements like quarts $(2.65 \mathrm{~g} / \mathrm{cc})$ and calcite $(2.71 \mathrm{~g} / \mathrm{cc})$. This affects bulk 
density measurements immensely, leading to open hole $(\mathrm{OH}) \operatorname{logs}$ that show erroneously high density porosity in organic-rich intervals (Passey et al. 2010). Evaluators should take note when looking at total organic carbon (TOC) values as well. Labs often cite TOC in weight percent (wt $\%$ ) rather than volume percent (vol\%), regardless of the fact that vol\% more closely reflects the actual reservoir rock volume taken up by OM (Passey et al. 2010). TOC values listed in vol\% will generally equal approximately double their corresponding value in wt\% (Passey et al. 2010).

Within the context of the Eagle Ford shale, the role of OM is uniquely low relative to other systems such as the Barnett shale. SEM studies on Eagle Ford core samples have revealed that though OM is certainly present in the Eagle Ford, its pore network is still dominated by pores in the inorganic matrix (Ko et al. 2017; Pommer and Milliken 2015). Pommer and Milliken (2015) showed that most OM porosity exists in isolated bodies of OM that are not necessarily connected. Interestingly, they also showed that rock samples with higher maturity organic matter might tend more towards hosting pore networks dominated by OM in lieu of the mineral grains. That said, Eagle Ford TOC values still compare closely with many other basins, generally sitting within the range of 3-7\% (Zou 2017). 


\section{Reservoir Fluids and OM Maturity}

Producible shales are ultimately producible source rocks, so the maturity of the contained organic matter drives the type of fluids that said source rock contains (Bohacs et al. 2013; Jarvie 2012a, 2012b; Passey et al. 2010). Less mature OM is prone to generating oil, and more mature OM is prone to generating gas (Bohacs et al. 2013; Jarvie 2012a, 2012b). Oil-OM also tends to contain less porosity than gas-prone OM simply because it tends to be less mature, meaning less of it has converted to fluid hydrocarbons than (Bohacs et al. 2013).

These observations are consistent with observations made by Yao Tian (2013) regarding the fluid typing and OM maturity of the Eagle Ford. The Eagle Ford produces a wide array of fluids. In the northwest, the Eagle Ford has low-maturity OM and mainly produces oil (Yao Tian 2013). Along its center we see medium-maturity OM and a combination of oil production and condensate production (Yao Tian 2013). In the southeast, the Eagle Ford has highly mature OM and it primarily produces dry gas (Yao Tian 2013). 


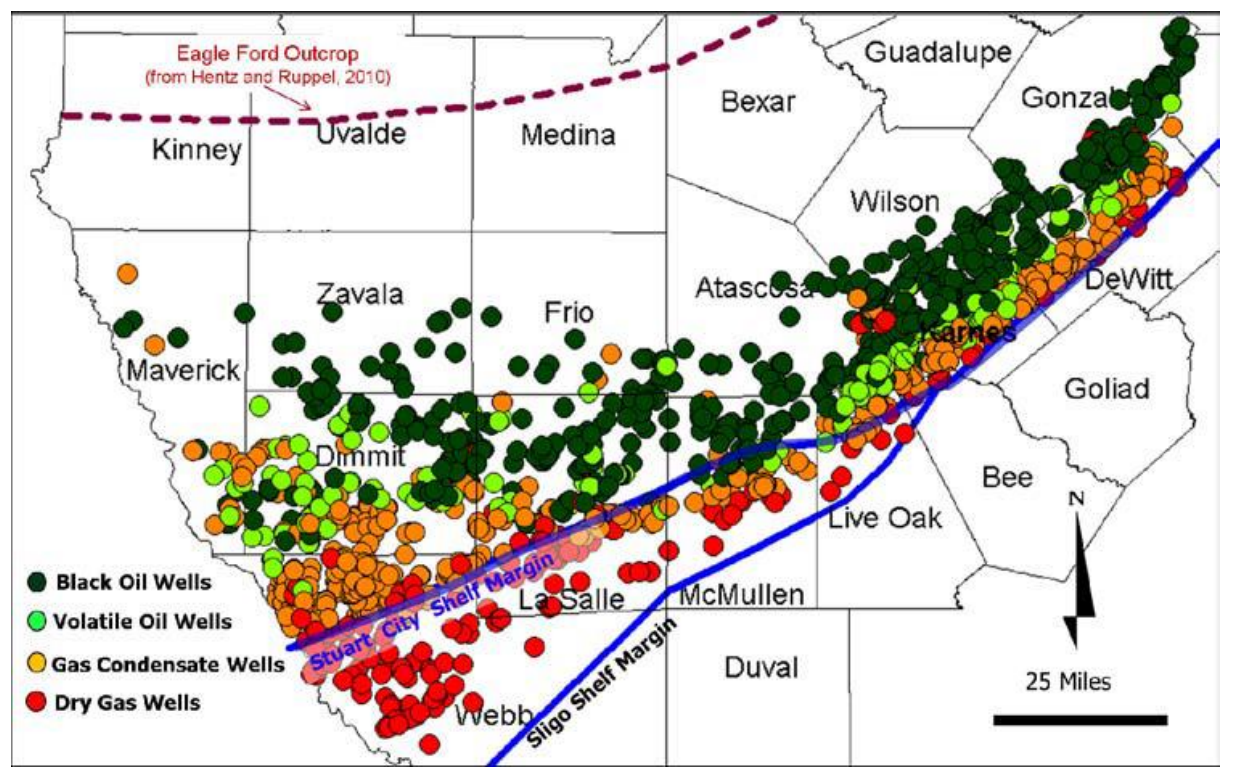

Figure 4 - Eagle Ford Shale Fluid Production Map - From Yao Tian (2013) Republished by permission of the Gulf Coast Association of Geological Societies, whose permission is required for further publication use

\section{Non-Darcy Flow}

Given the extremely small grain size of shales, pore throats tend to be at the nano-scale as well (Nelson 2009). In many shales, pores are small enough that pore throats begin to approach the size of a single molecule of oil, and in some cases even the size of a single molecule of methane (Blasingame 2008; Nelson 2009). Many authors argue that this gives rise to a need for alternatives to Darcy's Law in both shale gas and shale oil reservoirs.

Javadpour (2009) describes the nature of flow in nano-pores and brings up the relevance of Knudsen diffusion and no-slip flow in gas flow through shales. Knudsen diffusion is a phenomenon whereby diffusive flow is accelerated in conduits where the 
mean free path of a molecule is longer than the diameter of the conduit. Javadpour (2009) points out that erroneously high flow rates observed in shale gas wells could be caused by Knudsen diffusion and slip-free flow and present a flow expression in the form of Darcy's Law that corrects for these phenomena. This expression gives rise to the property "apparent permeability", which describes an equivalent Darcy permeability to gas, corrected for the effects of Knudsen diffusion and no-slip flow

Wasaki and Akkutlu (2015) took another approach and argued that gas flow in shales is heavily influence by the effects of gas adsorption to organic matter, and by local phase changes near pore throat walls caused by the varying density of gas in a single nano-pore could accelerate flow rates. They also presented an alternative to Darcy's Law with validation and presented another description of "apparent permeability".

Less work has been done on shale oil reservoirs, but Wang et al. (2016) presents the argument that the slip-free flow assumption in Darcy's Law also fails for oil flow. They present methods for compensating for no-slip flow when using Darcy's Law.

It is well established that the Eagle Ford has both micro- and nano-pores (Ko et al. 2017; Mullen 2010; Pommer and Milliken 2015), so the possibility of non-Darcy flow in the Eagle Ford is very real. Given that the Eagle Ford produces oil, condensate, and dry gas from rocks with micro- and nano-darcy permeability, fluid flow modeling in the Eagle Ford could become extremely complex if evaluators wish to correct for all the possible non-Darcy flow effects. Fortunately, many commercial simulators allow for non-Darcy flow corrections, and any modeling effort in the Eagle Ford should at least 
include a sensitivity study of the different non-Darcy flow effects that hold relevant for a given area.

\section{Sorption Effects}

Original gas in place (OGIP) estimations in shale gas reservoirs see the effects of organic matter through distortions in volumetric calculations that have little to do with the actual pore volume of a rock (Ambrose et al. 2012). Shale gas reservoirs store gas as free gas in pores and cracks, but also as an adsorbed phase on the surfaces of OM and clay material, and as an absorbed, or "diffuse", phase within the molecular structure of OM (Ambrose et al. 2012; Sondergeld et al. 2010a). Because much of the sorbed gas phase is irrecoverable, OGIP calculations mislead evaluators who account for the entirety of OM-hosted porosity in shale reservoirs (Ambrose et al. 2012). This is particularly relevant in unconventional reservoirs because the ratio of surface area to pore volume is very high in nano-scale pore networks, meaning a substantial component of stored gas might be adsorbed to organic pore walls and clay material (Ambrose et al. 2012). Ambrose et al. (2012) proposed an OGIP calculation methodology that corrects for the effects of adsorption, and Hartman et al. (2011) expounds upon this work by investigating the effects of adsorption in the context of multi-component (not exclusively methane) gas storage.

Sorption effects do not exclusively affect gas reservoirs. Oil adsorbs to organic matter much like gas, and this adsorption affects both the storage and flow of oil in shale reservoirs (Jarvie 2012b; Wang et al. 2015; Wang et al. 2016). Wang et al. (2015) even 
argues that a given quantity of organic matter will adsorb oil in greater quantities than it would gas. Adsorption more drastically affects heavier phases of oil (Wang et al. 2015). This being said, productive shale oil reservoirs tend to have high permeability relative to their shale gas cousins because higher permeability is required to produce higher viscosity fluids, and higher permeability ties into larger pores (Jarvie 2012b; Nelson 2009). In addition, many shale oil reservoirs fall into the aforementioned "hybrid reservoir" category, and have lower TOC than shale gas reservoirs, giving oil less surface area to adsorb to.

The Eagle Ford reservoir produces a wide spectrum of fluids - dry gas, wet gas, condensate, and black oil (Tian et al. 2013), and would most likely see the effects of adsorption in organic-rich facies that produce gas phases (ie. gas-producing LEF intervals). OGIP numbers from the Eagle Ford should likely take this into consideration. Oil-producing intervals could certainly see the effects of adsorption, however, this would be a function of TOC and oil composition.

\section{Category 3 - Completion Parameters}

Multi-stage hydraulic fracturing along horizontal wellbores is the keystone technology that enabled production from unconventional reservoirs (Clarkson et al. 2016). Fractures provide a flow conduit to the wellbore through formation with a permeability far in excess of the surrounding rock matrix. Hydraulic fracturing technology's development through the shale boom drives what is ultimately economic and uneconomic, and our understanding of how our fractures interact with the formation 
is critical when designing hydraulic fracture stimulation programs. In this paper, we do not attempt to discuss the design of hydraulic fracturing treatments in depth. Instead we focus on how the pre-existing conditions of the formation affect hydraulic fracture propagation, and the ramifications of different resulting fracture geometries.

\section{Natural Fractures}

We established earlier that shale reservoirs often contain an abundance of natural fractures (Gale et al. 2014). When hydraulic fractures (HF's) propagate through naturally-fractured rock, they interact with pre-existing natural fractures (NF's) in a variety of ways. Dahi Taleghani and Olson (2013) documents three possibilities. In the case where a HF propagates normally to a NF, it can simply cross through, unperturbed (Dahi Taleghani and Olson 2013). At shallower angles, hydraulic fracturing fluids may also divert into the NF, re-opening it and likely filling it with proppant, ultimately including in the final fracture network that connects to the wellbore (Dahi Taleghani and Olson 2013). NF's can also cause a propagating HF to "split" as a refractive process (Dahi Taleghani and Olson 2013). Fracture propagation models (FPM's) such as the ones described in Wu and Olson (2016) and Li et al. (2018) provide reservoir evaluators with powerful predictive tools that can generate complex fracture models based upon a user-input pump schedule and geologic model. 


\section{Rock Mechanical Properties}

Cipolla et al. (2011) describes the appraisal of unconventional reservoirs and highlights the importance of evaluating what they call "completion quality", defined as a formation's tendency to allow the initiation, propagation, and maintenance of conductive hydraulic fractures. Completion quality encompasses a variety of rock properties, the foremost of which are the in-situ principal stresses, Poisson's ratio, and Young's modulus (Cipolla et al. 2011).

Hydraulic fractures propagate perpendicular to the minimum horizontal stress direction in a formation, and the magnitude of each principal stress controls the pressure required to make a fracture propagate (Gidley et al. 1990). In a stress field with greater stress anisotropy, fracture propagation paths are more obvious and certain than in isotropic stress fields (Cipolla et al. 2011). Poisson's Ratio tells us the expected expansion in a material along one axis as a function of the compression of the same material along a perpendicular axis. This can be used to compute horizontal stresses as a function of vertical stresses (Gidley et al. 1990). Young's modulus tells us about the stiffness of the rock - a high Young's modulus ties into high stiffness and narrower fractures, whereas a low Young's modulus ties into low stiffness and wide fractures (Gidley et al. 1990).

Another mechanical component that has gained traction in the unconventional reservoir sphere is mineralogy. With the common abundance of clays in shales, rocks can have high ductility and thus present resistance to the initiation of hydraulic fractures (Sondergeld et al. 2010b). Jarvie et al. (2007) and Rickman et al. (2008) present the 
"brittleness index" (BI) (1.1) that describes the brittleness (and thus tendency to allow fracture initiation) as a function of mineralogy. Quartz-heavy rocks are commonly very brittle, clay-heavy rocks are commonly very ductile, and carbonate-heavy rocks fall somewhere in the middle (Sondergeld et al. 2010b).

$$
B I(\%)=\frac{\text { Quartz }}{\text { Quartz }+ \text { Carbonate }+ \text { Clays }}
$$

The Eagle Ford shale is a marine marl formation, heavy in carbonate with varying levels of clay and quartz content (Donovan and Staerker 2010; Ko et al. 2017; Lock et al. 2010). It has seen numerous tectonic events that led to natural fracturing (Ferrill et al. 2014) and will have varying stress profiles depending on proximity to salt bodies and tectonic folds throughout the basin. The informed evaluator would certainly want to consider rock mechanical properties and mineralogy when designing hydraulic fracture programs in the Eagle Ford. Where possible, horizontal wells should be drilled along the minimum horizontal stress direction to allow maximum hydraulic fracture propagation coverage, and fractures should be targeted in zones with higher brittleness and lower Young's modulus.

\section{Fracture/Stage Spacing}

Multi-stage hydraulically fractured horizontal wells enable production from shale reservoirs, but designing these wells introduces a new set of questions that have to do with their design. Operators must decide on a lateral section length along with the combined design of hydraulic fracturing and perforation parameters. Perforations are 
typically shot in "clusters", and isolated via plugs during "plug-and-perf" operations, and subsequently hydraulically fractured as a single unit. Smaller perforation clusters are preferred when attempting to initiate "planar" fractures, and thicker perforation clusters are preferred when attempting to initiate "network" fractures.

Perforation cluster and fracturing spacing optimization is a field of study of its own, but at its core, its goal is to optimize costs to provide the cheapest possible well for the greatest possible productivity by initiating fractures at an optimal distance from each other by placing perforation clusters at optimum distances from each other. Mayerhofer et al. (2010) tells us that the optimal stage spacing is that which generates a large "stimulated reservoir volume" (SRV) with a high density of hydraulic fractures.

\section{Fracture Network Geometry}

Classical hydraulic fracture fluid flow theory looks at fractures as single planar entities, mostly as a simplifying assumption to enable derivation of fluid flow principles (Economides et al. 2013; Lee et al. 2003). Planar models certainly seem too good (or simple) to be true, but many authors have shown that planar models provide a foundation for useful production analysis in unconventional reservoirs (Bello and Wattenbarger 2010; Chen and Rajagopal 1997; Samandarli et al. 2012; Song et al. 2011; Wattenbarger et al. 1998). That said, it would be naïve to assume that fractures simply follow a planar bi-wing geometry in the presence of variable stress fields, anisotropic and heterogeneous rock properties, and natural fractures. 
Microseismic studies provide very strong evidence that hydraulically fracturing a rock formation actually produces a complex network of fractures as the combined results of non-uniform hydraulic fracture propagation and the reactivation of pre-existing natural fractures (Cipolla et al. 2011; Cipolla et al. 2009; Mayerhofer et al. 2010; Warpinski et al. 2008). Accounting for the complexity of network hydraulic fractures is beyond the scope of classical analytic models but can be done with more complex 3dimensional numerical reservoir models. Perpendicular bisector (PEBI) grids provide one avenue for modeling complex fracture networks, and allow evaluators to represent fractures discretely as actual grid blocks on a simulation grid (Sun and Schechter 2016). Though these simulations can be computationally expensive, they enable the explicit testing of properties like stress-dependent permeability and fracture aperture distributions through the modification of fracture cell properties (Sun and Schechter 2016; Sun et al. 2016). Alternatively, embedded discrete fracture model (EDFM) based techniques provide a slightly simpler, albeit less rigorous way to model complex fracture networks in unconventional reservoirs (Li et al. 2018).

\section{Stimulated Reservoir Volume (SRV)}

Whether through micro-fractures that are undiscernible in microseismic surveys, or through some sort of intrinsic permeability stimulation, the SRV has become a nearly ubiquitous piece of any unconventional reservoir model. Typically, the SRV is simply a rectangular region of rock, placed around the whole well or around individual fractures, 
where evaluators assume that permeability is enhanced by some multiple of the prefracturing rock matrix permeability (Clarkson et al. 2009).

Mayerhofer et al. (2010) expounds upon the SRV concept with evidence from microseismic surveys, claiming that the SRV is just a way to represent highly complex fracture networks. They draw comparisons between complex fracture networks and enhanced-permeability rocks, and propose that the estimation of SRV pore volume through microseismic surveys can provide a powerful volumetric tool (Mayerhofer et al. 2010).

The SRV concept has implications on shale production analysis as well. In the field, operators have observed that shale wells typically flow under transient bilinear or linear conditions for years before eventually exhibiting the "boundary-dominated" flow (BDF) behavior in pressure and rate data (Anderson et al. 2010; Clarkson et al. 2009; Duong 2010; Yu et al. 2013). The onset BDF represents the beginning of interference between fractures, where fractures' drainage areas grow large enough to affect their neighbors (Anderson et al. 2010).

\section{Conclusions}

In this chapter we presented a review of current literature in the field of unconventional reservoir geology, petrophysics, and engineering, and topics that we believe should be considered in any unconventional reservoir study. We contextualized these topics as they pertain to the Eagle Ford reservoir of south Texas and provide this 
work as a framework for future studies in unconventional reservoirs. We provide a summary of these topics in Figure 1.

For the remainder of this document, we will focus on a subset of challenges namely those that have to do with the flow of reservoir fluids to multi-stage hydraulically-fractured horizontal wells (Figure 5). In addition, we provide a focus on the onset of fracture interference. The onset of fracture interference represents a critical time in unconventional reservoir evaluation. The detection of the onset of this interference can be used to calculate the permeability of rock between the fractures, and to evaluate the volume of an SRV (Song et al. 2011; Song and Ehlig-Economides 2011). In Chapters 2 and 3, we will discuss the implications of fracture interference and the time at which it begins (referred to as the "end of linear flow" $\left(t_{e l f}\right)$ ).

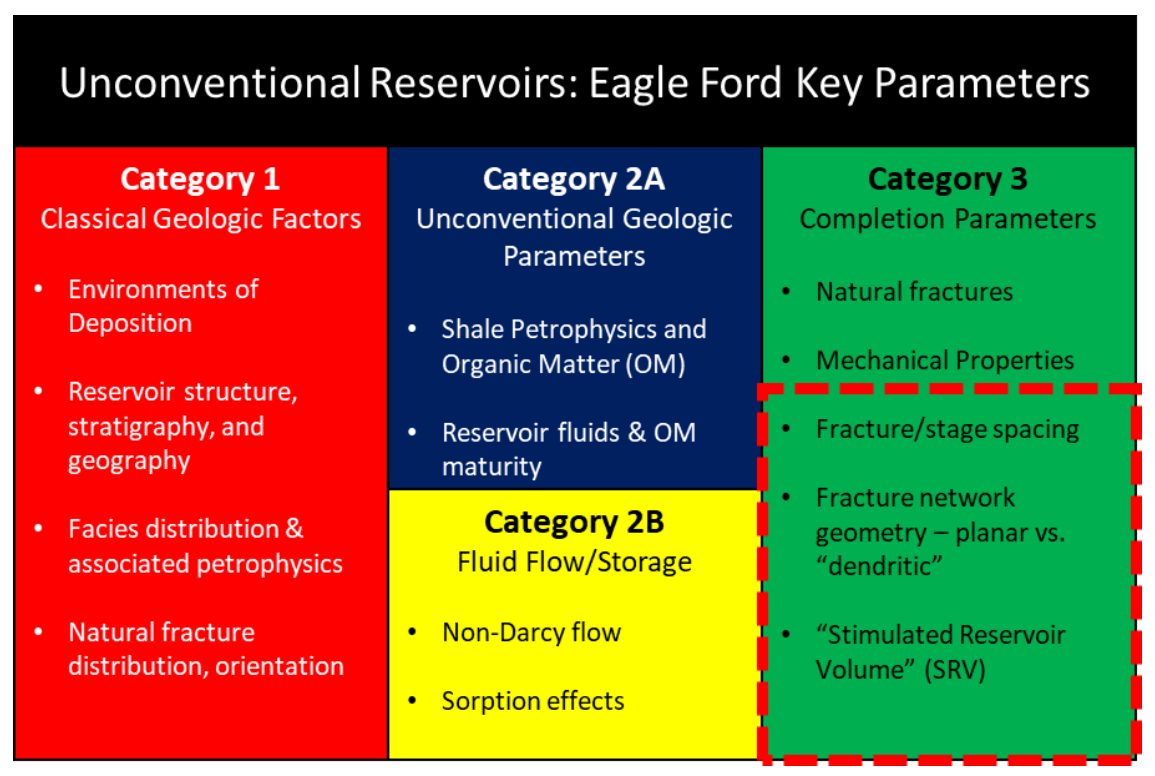

Figure 5 - Unconventional Reservoir Framework - This study's focus highlighted in red box 


\section{CHAPTER II}

\section{DEVELOPMENT OF ANALYTIC MTFW SOLUTIONS*}

\section{MTFW Production Analysis Introduction}

Economic production from shale gas and shale oil resources almost universally requires the drilling and completion of multi-stage hydraulically-fractured horizontal wells (Clarkson 2013). This technology has been in development for decades, but the industry's understanding of the physical phenomena in play still has much to gain. Between advances in decline curve production forecasting, improvements in 3-d reservoir models, and the development of various analytical models, our understanding of the fluid flow phenomena in unconventional shale developments has improved over time.

This chapter focuses on the development of production analysis and rate transient analysis (RTA). As a starting point for the rate transient field, Palacio and Blasingame (1993) constructed a new type curve based upon the Fetkovich type curve (Fetkovich 1980). Palacio and Blasingame (1993) showed that when using material balance time ( $t_{m b}$ ) instead of actual time, a series of transients with varying rate and pressure can be reduced to the harmonic solution (or stem) of the Fetkovich type curve. Palacio and Blasingame (1993) focused on the development of type curves for the interpretation of long-term transient flow for a variety of geometries under varying-rate inner boundary

\footnotetext{
* Reprinted with permission from "Characterization of Multiple Transverse Fractured Wells Using the Asymptotic Approximation of the Diffusivity Equation” by Andrew Malone, Michael J. King, and Zhenzhen Wang, 2019. SPE Europec featured at the $81^{\text {st }}$ EAGE Conference and Exhibition, Copyright [2019] by the Society of Petroleum Engineers
} 
conditions, however their study made it clear that diagnostic plots can also be built and interpreted for non-constant well rate production data but interpreted using the same principles we use for constant rate data.

Unconventional shale oil and gas developments almost necessitate the use of rate-transient techniques in lieu of pressure-transient analysis. Most shale reservoirs have permeabilities on the order of 10 to 100 nanodarcies (Cipolla et al. 2009). Given this ultra-low permeability, transient flow periods can persist through the entire life cycle of a well. In such situations, classic pressure-transient methodologies cannot be applied for the determination of rock properties and reservoir size, and rate-transient analysis can play a part.

Song et al. (2011) investigated the progression of flow regimes for MTFW wells, and how the interaction of pressure transients between multiple transverse fractures will affect a long-term production response at the wellhead. They identified the fact that hydraulic fractures initially behave independently, exhibiting linear or bilinear earlytime flow. Eventually, fracture drainage areas will begin to overlap, and no-flow boundaries develop between the fractures. At this point, a period boundary-dominated flow begins, which shows up as a unit slope on the derivative curve (in this case RNP') of a diagnostic plot. Song et al. (2011) coined the term "pseudo pseudo-steady state" to describe this phenomenon and showed how this new flow regime may be used to describe reservoir and completion properties for a MTFW.

Song and Ehlig-Economides (2011) elaborated on the work in Song et al. (2011), and described how rate-normalized pressure (RNP) and its derivative (RNP') may be 
used in conjunction with the material balance time concept to build equivalent constantrate diagnostic plots from varying-rate and varying-pressure production data. They show that as long as the progression of flow regimes described in Song et al. (2011) is observed, shale permeability and stimulated reservoir volume (SRV) may be calculated using rate-transient analysis.

One shortcoming of the methodology described in Song and Ehlig-Economides (2011) is the determination of the "end of linear flow". Their methodology defines the SRV as a finite region that fully describes the drainage volume of a well at the point in time when "pseudo pseudo-steady state" flow begins. The identification of the beginning of this flow regime and the associated interpretation can drastically affect the calculation of shale matrix permeability and SRV volume.

Wattenbarger et al. (1998) provides some work on a different type of reservoir tight gas - that can be used in the context of MTFW's. Wattenbarger et al. (1998) tells us that many tight gas wells are drilled as vertical wells with single vertical hydraulic fracture completions and will experience long-term linear flow followed by boundarydominated or pseudo-steady state flow. This is analogous to the MTFW case, where we see early-time linear or bilinear flow (with fractures acting independently) followed by “pseudo pseudo-steady state" flow. Wattenbarger et al. (1998) developed an analytic model and type curve that describes this behavior by placing a planar pressure sink in a rectangular drainage area. This type curve describes the flow to a single fracture but has no way of accounting for the level of interaction that occurs between hydraulic fractures during production. 
In this chapter we construct our own analytic model to describe the behavior of MTFW's for the simplifying single-phase oil flow case. Utilizing the asymptotic approximation of the diffusivity equation as described in King et al. (2016), we propose both 1-dimensional and 2-dimensional flow models for MTFW's under constant wellbore rate, constant bottom hole pressure, and variable rate constraints. In this chapter we describe the background mathematics and specific formulations used to construct this set of solutions. We validate each case against a commercial simulator base case and describe the nature of fracture interference and SRV drainage as dictated by our model. In the following chapter (Chapter 3), we will investigate the methods for determining the end of linear flow in MTFW's, and how this connects to our analytic MTFW model. 


\section{Asymptotic Approximation of the Diffusivity Equation}

\section{Base Theory}

King et al. (2016) introduced the asymptotic approximation of the diffusivity equation in its current form with the goal of reducing 3-dimensional reservoir flow problems in the domain $(x, y, z)$ to 1 -dimensional flow problems defined in terms of the "diffusive time of flight", $(\tau(\vec{x}))$. Their formulation begins with the generalized form of the 3-dimensional reservoir diffusivity equation (Eq. (2.1)).

$$
\phi(\vec{x}) \mu c_{t} \frac{\partial p(\vec{x}, t)}{\partial t}=\nabla \cdot[\overrightarrow{\vec{k}}(\vec{x}) \cdot \nabla p(\vec{x}, t)]
$$

In lieu of the classical Laplace transform methodology, we can take the Fourier transform of Eq. (2.1), yielding the diffusivity equation in the frequency domain.

$$
\phi(\vec{x}) \mu c_{t}(-i \omega) p(\vec{x}, \omega)-\nabla \cdot[\overrightarrow{\vec{k}}(\vec{x}) \cdot \nabla p(\vec{x}, \omega)]=0
$$

The frequency domain solution is provided by Virieux et al. (1994) (Eq. ).

$$
p(\vec{x}, \omega)=e^{-\sqrt{-i \omega \tau}(\vec{x})} \sum_{k=0}^{\infty} \frac{A_{k}(\vec{x})}{(\sqrt{-i \omega})^{k}}
$$

We then take the high-frequency limit $(\omega \rightarrow \infty)$ of Eq. (2.3), which corresponds to the propagation of a sharp front. In this case, that sharp front is the front of a "pressure wave". The high-frequency limit gives rise to Eq. (2.4), Eq. (2.5), and Eq. (2.6).

$$
\nabla \tau(\vec{x}) \cdot \overrightarrow{\vec{k}}(\vec{x}) \cdot \nabla \tau(\vec{x})=\phi(\vec{x}) \mu c_{t}
$$




$$
\begin{gathered}
p(\vec{x}, t) \approx p(\tau(\vec{x}), t) \\
\frac{\partial p}{\partial t} \sim e^{-\tau^{2} / 4 t}
\end{gathered}
$$

Equation (2.4) is the Eikonal Equation (Kulkarni et al. 2001), and it describes the development of the contours of $\tau$ with respect to the reservoir diffusivity, $\alpha$. Equation (2.5) tells us that the contours of pressure will follow the shape of the contours of $\tau$. Finally, Equation (2.6) expresses the time derivative of pressure as proportional to the time-domain form of the exponential term from (2.3).

The Eikonal Equation (Eq. (2.4)) may be solved numerically in heterogeneous reservoir cases using the Fast Marching Method (Zhang et al. 2013). For homogeneous reservoirs, we can solve Eq. (2.4) simply with (2.7).

$$
\begin{gathered}
\tau=\frac{r}{\sqrt{\alpha}} \\
\alpha=\frac{k}{\phi \mu c_{t}}
\end{gathered}
$$

In field units,

$$
\alpha=\frac{k}{3792 \phi \mu c_{t}}
$$

From this point, we may express Darcy's Equation in terms of $\tau$ with Eq. (2.11), and diffusivity in terms of $\tau$ with Eq. (2.10)

$$
c_{t} \frac{\partial p(\tau, t)}{\partial t}=\frac{1}{w(\tau)} \frac{\partial q(\tau, t)}{\partial \tau}
$$




$$
q(\tau, t)=c_{t} w(\tau) \frac{\partial p(\tau, t)}{\partial \tau}
$$

The function $w(\tau)$ also warrants an individual explanation. $w(\tau)$ is the derivative of the pore volume $\left(V_{p}\right)$ with respect to $\tau$.

$$
w(\tau)=\frac{d V_{p}(r)}{d \tau}
$$

$V_{p}$ is the total pore volume of the drained area, determined primarily by drainage pattern or flow regime. For example, with radial flow, we have

$$
V_{p}(r)=\pi r^{2} h \phi
$$

Finally, we recall Equation (2.6) and represent the time derivative of pressure as an exponential term multiplied by an unknown pre-factor, $A(\tau, t)$ (Equation (2.14)), which we approximate with a power series (Equation (2.15)).

$$
\begin{gathered}
\frac{\partial p}{\partial t} \approx-A(\tau, t) e^{-\tau^{2} / 4 t} \\
A(\tau, t)=\left\{\sum_{n=0}^{\infty} \tau^{n} A_{n}(t)\right\}
\end{gathered}
$$

Now we insert these expressions into Eq. (2.10), allowing us to rewrite the diffusivity equation in terms of $\tau$ with a third term (Eq. (2.16).

$$
c_{t} \frac{\partial p(\tau, t)}{\partial t}=\frac{1}{w(\tau)} \frac{\partial q(\tau, t)}{\partial \tau}=-\left\{\sum_{n=0}^{\infty} \tau^{n} A_{n}(t)\right\} e^{-\tau^{2} / 4 t}
$$


Equation (2.16) equates the pressure derivative at any given point in time and $\tau$ to the flux derivative with respect to $\tau$ and the infinite series listed on the far right side of Equation (2.16). This infinite series is referred to as the "asymptotic expansion" and provides a simple tool for constructing diffusivity relationships for a long list of flow regimes and boundary conditions. The infinite series term sums up of the increasing powers of $\tau$ multiplied by an unknown function, $A_{n}(t)$. This series may be truncated, giving us an estimation of the left-hand terms, rather than an exact solution. The number of terms required for this series varies by problem, but King et al. (2016) shows that 2term approximations sufficiently resolve most flow problems. In this study, we limit ourselves to 1- and 2-term approximations. In the next subsections, we describe how the asymptotic approximation may be used to describe either flux or pressure at the wellbore or any point in the reservoir.

\section{Asymptotic Approximation - Flux}

To describe wellbore flow, we rearrange Eq. (2.16) and take the first integral.

$$
\begin{gathered}
\frac{1}{w(\tau)} \frac{\partial q(\tau, t)}{\partial \tau}=-\left\{\sum_{n=0}^{\infty} \tau^{n} A_{n}(t)\right\} e^{-\tau^{2} / 4 t} \\
\int_{\tau=0}^{\tau=\infty} \partial q(\tau, t)=-\int_{\tau=0}^{\tau=\infty} w(\tau)\left\{\sum_{n=0}^{\infty} \tau^{n} A_{n}(t)\right\} e^{-\tau^{2} / 4 t} \partial \tau
\end{gathered}
$$


We refer to flux at the sand face as equal to well rate, and hence label it $q_{w}$. We assume infinite-acting flow and say $q \rightarrow 0$ as $\tau \rightarrow \infty$. Because of this, and because $q \rightarrow q_{w}$ as $\tau \rightarrow 0,(2.18)$ evaluates to

$$
q_{w}(t)=\int_{\tau=0}^{\tau=\infty} w(\tau)\left\{\sum_{n=0}^{\infty} \tau^{n} A_{n}(t)\right\} e^{-\tau^{2} / 4 t} \partial \tau
$$

Rearrange and we have the following expression.

$$
q_{w}(t)=\sum_{n=0}^{\infty} A_{n}(t) \int_{\tau=0}^{\tau=\infty} w(\tau) \tau^{n} e^{-\tau^{2} / 4 t} \partial \tau
$$

For the purpose of simplification, we define the "volume moment integral", $V_{n}(\tau, t)$ as the following.

$$
V_{n}(\tau, t)=\int_{\tau^{\prime}=\tau}^{\tau^{\prime}=\infty} w\left(\tau^{\prime}\right) \tau^{\prime n} e^{-\tau^{\prime 2} / 4 t} \partial \tau^{\prime}
$$

Since we are describing the flux at the wellbore $(\tau=0)$, this becomes $V_{n}(0, t)$, or just $V_{n}(t)$, and can be simplified (2.22). This expression defines the transient drainage volume of the well (or of the pressure sink located at $\tau=0$ ) (Wang et al. 2018).

$$
V_{n}(t)=V_{n}(0, t)=\int_{\tau=0}^{\tau=\infty} w(\tau) \tau^{n} e^{-\tau^{2} / 4 t} \partial \tau
$$

We can now rewrite (2.20) as the product of volume moment integrals and unknown functions. This provides further simplification for use when deriving specific expressions for flux at the wellbore. 


$$
V_{n}(t)=V_{n}(0, t)=\int_{\tau=0}^{\tau=\infty} w(\tau) \tau^{n} e^{-\tau^{2} / 4 t} \partial \tau
$$

For flux at the wellbore, we have

$$
q_{w}(t)=\sum_{n=0}^{\infty} A_{n}(t) V_{n}(t)
$$

Away from the wellbore, we also have

$$
q(\tau, t)=\sum_{n=0}^{\infty} A_{n}(t) V_{n}(\tau, t)
$$

\section{Asymptotic Expansion - Pressure Drawdown}

To obtain a pressure drop expression, we first rearrange Eq. (2.11)

$$
c_{t} \frac{\partial p(\tau, t)}{\partial \tau}=\frac{1}{w(\tau)} q(\tau, t)
$$

Rearrange again and integrate, and we have

$$
c_{t} \int_{\tau^{\prime}=\tau}^{\tau^{\prime}=\infty} \partial p\left(\tau^{\prime}, t\right)=\int_{\tau^{\prime}=\tau}^{\tau^{\prime}=\infty} \frac{1}{w\left(\tau^{\prime}\right)} q\left(\tau^{\prime}, t\right) \partial \tau^{\prime}
$$

Because of our infinite-acting flow assumption, we can say $p \rightarrow p_{i}$ as $\tau \rightarrow \infty$.

$$
c_{t} \Delta p(\tau, t)=\int_{\tau^{\prime}=\tau}^{\tau^{\prime}=\infty} \frac{1}{w\left(\tau^{\prime}\right)} q\left(\tau^{\prime}, t\right) \partial \tau^{\prime}
$$

Using (2.16) we rewrite this as

$$
c_{t} \Delta p(\tau, t)=\int_{\tau^{\prime}=\tau}^{\tau^{\prime}=\infty} \frac{1}{w\left(\tau^{\prime}\right)} \int_{\tau^{\prime \prime}=\tau^{\prime}}^{\tau^{\prime \prime}=\infty} w\left(\tau^{\prime \prime}\right)\left\{\sum_{n=0}^{\infty} \tau^{n n} A_{n}(t)\right\} e^{-\tau^{\prime \prime 2} / 4 t} \partial \tau^{\prime \prime} \partial \tau^{\prime}
$$


Rearrange to get

$$
c_{t} \Delta p(\tau, t)=\sum_{n=0}^{\infty} A_{n}(t) \int_{\tau^{\prime}=\tau}^{\tau^{\prime}=\infty} \frac{1}{w\left(\tau^{\prime}\right)} \int_{\tau^{\prime \prime}=\tau^{\prime}}^{\tau^{\prime \prime}=\infty} w\left(\tau^{\prime \prime}\right) \tau^{\prime \prime n} e^{-\tau^{\prime 2} / 4 t} \partial \tau^{\prime \prime} \partial \tau^{\prime}
$$

Again, for simplification, we define the "pressure drop moment integral", $W_{n}(\tau, t)$

$$
W_{n}(\tau, t)=\int_{\tau^{\prime}=\tau}^{\tau^{\prime}=\infty} \frac{1}{w\left(\tau^{\prime}\right)} \int_{\tau^{\prime \prime}=\tau^{\prime}}^{\tau^{\prime \prime}=\infty} w\left(\tau^{\prime \prime}\right) \tau^{\prime n} e^{-\tau^{n^{2}} / 4 t} \partial \tau^{\prime \prime} \partial \tau^{\prime}
$$

In terms of the volume moment integral,

$$
W_{n}(\tau, t)=\int_{\tau^{\prime}=\tau}^{\tau^{\prime}=\infty} \frac{V_{n}\left(\tau^{\prime}\right)}{w\left(\tau^{\prime}\right)} \partial \tau^{\prime}
$$

We can now rewrite (2.30) as the product of pressure drop moment integrals and unknown functions. This provides further simplification for use when deriving specific expressions for pressure drop at the wellbore and at locations in the reservoir. At the wellbore this becomes Equation (2.33).

$$
c_{t} \Delta p_{w f}=\sum_{n=0}^{\infty} A_{n}(t) W_{n}(t)
$$

Away from the wellbore, we have Equation (2.34).

$$
c_{t} \Delta p(\tau, t)=\sum_{n=0}^{\infty} A_{n}(t) W_{n}(\tau, t)
$$

\section{Inner Boundary Conditions}

Equations (2.25) and (2.34) provide us with powerful tools for constructing a 
variety of diffusivity relationships within a simple framework, primarily because of how well each piece of each equation connects to different components of a given flow system. The moment integrals, $V_{n}$ and $W_{n}$, are influence by the flow geometry of the system through the pore volume terms contained within the $w(\tau)$ function. Their integrands control the point at which pressure or flux is observed, and the extent of the flow system in the $\tau$ domain. Finally, the unknown functions, $A_{n}(t)$, are influenced by the inner boundary conditions of the system.

The connection between specific $A_{n}(t)$ terms and the derivatives of flux and pressure allows us to use the unknown functions to impose inner boundary constraints on our flow system. The derivative of flux at the wellbore with respect to time relates to the first unknown function, $A_{1}(t)$, as in Eq. (2.35). The derivative of pressure at the wellbore with respect to time relates directly to the zeroth unknown function, $A_{0}(t)$, as in Eq. (2.36).

$$
\begin{gathered}
\frac{d q_{w}(t)}{d t}=-w(0) A_{1}(t) \quad @ \tau=0 \\
c_{t} \frac{d \Delta p_{w f}(t)}{d t}=A_{0}(t) \quad @ \tau=0
\end{gathered}
$$

With this, we can set wellbore rate as constant by setting the first unknown function, $A_{1}(t)$, to zero. Another way to say this is we set a constant-rate IBC by setting $n=0$ in the asymptotic expansion term of Eq. (2.16). 
We may set wellbore bottom hole flowing pressure as constant by setting the zeroth unknown function, $A_{0}(t)$, to zero. In other words, we can set a constant-BHP IBV by setting $n=1, n \neq 0$ in the asymptotic expansion term of Eq. (2.16).

One last set of constraints exists, which includes one functional constraint we name "variable rate" and one non-functional constraint which we simply call "constant BHP”. When working with multiple fracture cases (as will be discussed later), we must define flow to all fractures independently as well as to the well. To have a functional solution, we cannot simply constrain $A_{0}(t)$ or $A_{1}(t)$ to zero for all fractures as we do for a single-fracture solution. As we will show, the derivative is constrained at the well level, not the individual fracture level. This gives rise to a second pair of IBC's, both of which say nothing of the derivatives of rate or bottom hole pressure, and which assume that the term, $A_{0}+\tau A_{1}$, may be approximated by either $A_{0}(t)$ or $\tau A_{1}(t)$ alone (Eq. and Eq. respectively).

$$
\begin{aligned}
& A_{0}+\tau A_{1} \approx A_{0} \\
& A_{0}+\tau A_{1} \approx \tau A_{1}
\end{aligned}
$$

Using these constraints allows for simpler solutions but introduces error. As we will show, the variable rate solution is worth using, however the 1-term constant-BHP constraint falls short in its ability to model wellbore flow properly. 


\section{Applying the Asymptotic Approximation to Specific Flow Geometries}

In Section 2.2 we went through the derivation of expressions for flux and pressure drawdown using the asymptotic approximation of the diffusivity equation. We concluded these derivations with the simplified forms shown by Equation (2.25) and Equation (2.34). These forms are hardly useful for any real scenario however, until the moment integrals, $V$ and $W$, and the unknown functions, $A_{n}$, have been determined. In this section we describe how these terms are obtained for both 1-dimensional and 2dimensional flow to a single hydraulic fracture. These solutions are not new but will help the reader understand how the asymptotic approximation may be used to derive classical diffusivity relationships. In addition, the moment integral terms derived in this section will be used in the development of the MTFW solutions described later in this chapter.

\section{1-Dimensional Solutions - Infinite-Acting Linear Flow (IALF)}

Consider a single infinite-conductivity planar fracture in an infinite homogeneous, isotropic medium (Figure 6). The fracture has height $h$ and is fullypenetrating (this means the fracture height equals the pay height, $h$ ). Since the fracture is infinitely-conductive, we consider it to be a planar source and thus it has a negligible width. Given that this is a 1-dimensional flow case, the fracture will drain perpendicular to its face only.

To construct a diffusivity relationship for this system, we begin by defining $\tau$ as the ratio of the perpendicular distance $r$ from the fracture (Figure 7) to the square root of the reservoir diffusivity, $\alpha$. 


$$
\tau=\frac{r}{\sqrt{\alpha}}
$$

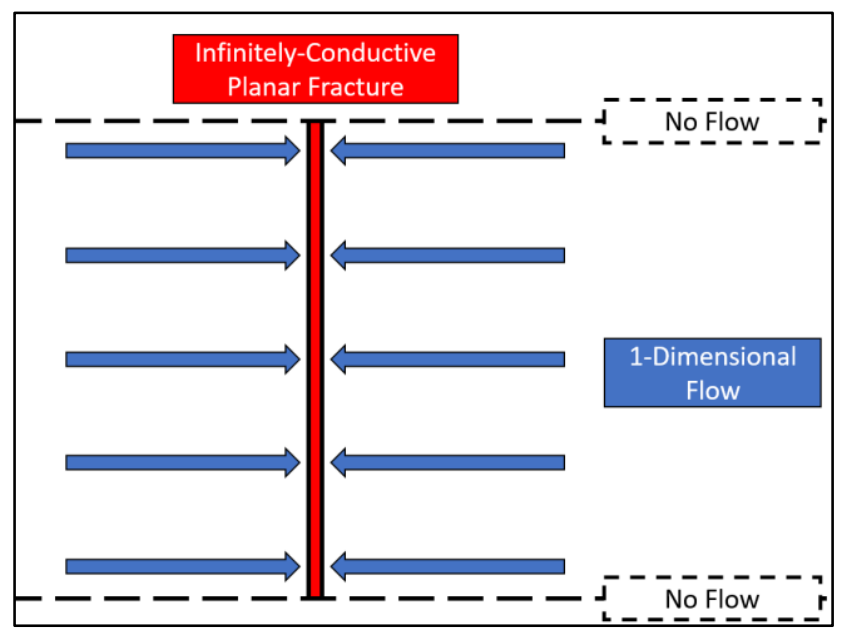

Figure 6 - IALF Diagram (after Malone et al. (2019))

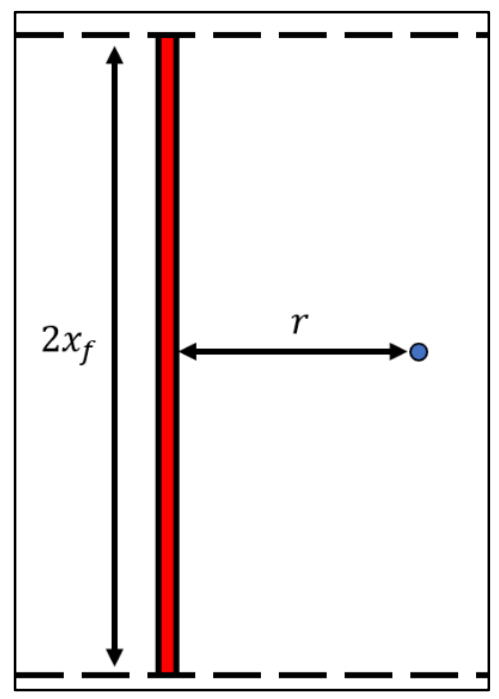

Figure 7 - IALF Coordinates (from Malone et al. (2019)). Copyright 2019, SPE. Reproduced with permission of SPE. Further reproduction prohibited without permission. 
We then define the pore volume of this system in terms of $r$ and $\tau$.

$$
\begin{gathered}
V_{p}(r)=A \phi r \\
V_{p}(\tau)=A \phi \tau \sqrt{\alpha}
\end{gathered}
$$

Note that $A$ is the cross-sectional area of flow, which in the planar fracture case will equal twice the area of the fracture.

$$
A=4 x_{f} h
$$

Now we take the derivative of pore volume with respect to $\tau$ and find $w(\tau)$.

$$
w(\tau)=\frac{d V_{p}(r)}{d \tau}=A \phi \sqrt{\alpha}
$$

Next the volume and pressure drop moment integrals must be calculated. For this study, we truncate the asymptotic expansion at 1 and 2 terms, so we use $n=0,1$.

$$
\begin{gathered}
V_{n}(\tau, t)=\int_{\tau^{\prime}=\tau}^{\tau^{\prime}=\infty} w\left(\tau^{\prime}\right) \tau^{\prime n} e^{-\tau^{\prime 2} / 4 t} \partial \tau^{\prime} \\
W_{n}(\tau, t)=\int_{\tau^{\prime}=\tau}^{\tau^{\prime}=\infty} \frac{V_{n}\left(\tau^{\prime}\right)}{w\left(\tau^{\prime}\right)} \partial \tau^{\prime} \\
V_{0}(\tau, t)=A \phi \sqrt{\pi \alpha t} \operatorname{erfc}\left(\frac{\tau}{\sqrt{4 t}}\right) \\
V_{0}(t)=A \phi \sqrt{\pi \alpha t}
\end{gathered}
$$




$$
\begin{gathered}
W_{0}(\tau, t)=2 t e^{-\tau^{2} / 4 t}-\sqrt{\pi t} \tau \operatorname{erfc}\left(\frac{\tau}{\sqrt{4 t}}\right) \\
W_{0}(t)=2 t \\
V_{1}(\tau, t)=2 t A \phi \sqrt{\alpha} e^{-\tau^{2} / 4 t} \\
V_{1}(t)=2 t A \phi \sqrt{\alpha} \\
W_{1}(\tau, t)=2 \sqrt{\pi t^{3}} e r f c\left(\frac{\tau}{\sqrt{4 t}}\right) \\
W_{1}(t)=2 \sqrt{\pi t^{3}}
\end{gathered}
$$

Using these expressions, we can arrive at the classical equations for IALF. We first assume a constant wellbore rate with single-phase slightly compressible liquid flow. To set our IBC, we constrain rate to a constant value by setting $A_{1}(t)$ to zero. Flux can then be expressed with Eq. (2.23).

$$
q_{w}=A_{0}(t) V_{0}(t)
$$

Plug in $V_{0}(t)$ and rearrange to arrive at an expression for the unknown function, $A_{0}(t)$.

$$
A_{0}(t)=\frac{q_{w}}{V_{0}(t)}=\frac{q_{w}}{A \phi \sqrt{\pi \alpha t}}
$$

Pressure drawdown may be calculated with (2.34).

$$
c_{t} \Delta p(\tau, t)=A_{0}(t) W_{0}(\tau, t)
$$




$$
c_{t} \Delta p(\tau, t)=\frac{q_{w}}{A \phi \sqrt{\pi \alpha t}}\left\{2 t e^{-\tau^{2} / 4 t}-\sqrt{\pi t \tau} \operatorname{erfc}\left(\frac{\tau}{\sqrt{4 t}}\right)\right\}
$$

Rearrange, and write this equation in terms of $r$ instead of $\tau$, and we have the same form as listed by Lee et al. (2003).

$$
\Delta p(r, t)=\frac{2 q_{w}}{A \phi c_{t}} \sqrt{\frac{t}{\pi \alpha}}\left\{e^{-r^{2} / 4 \alpha t}-\frac{r}{2} \sqrt{\frac{\pi}{t \alpha}} \operatorname{erfc}\left(\frac{\tau}{\sqrt{4 t}}\right)\right\}
$$

In dimensional form, this is

$$
\Delta p(r, t)=16.26 \frac{q_{w}}{A \phi c_{t}} \sqrt{\frac{t}{\pi \alpha}}\left\{e^{-r^{2} / 4 \alpha t}-\frac{r}{2} \sqrt{\frac{\pi}{t \alpha}} \operatorname{erfc}\left(\frac{\tau}{\sqrt{4 t}}\right)\right\}
$$

For the constant bottom hole pressure (constant-BHP) case, we instead set $A_{0}(t)$ to zero to constrain the pressure derivative at the wellbore. For the pressure drawdown expression, we use Eq. (2.34).

$$
c_{t} \Delta p(\tau, t)=A_{1}(t) W_{1}(\tau, t)
$$

With bottom hole pressure (BHP) constrained, we set $\tau$ to zero, and have

$$
c_{t} \Delta p_{w f}=A_{1}(t) W_{1}(t)
$$

Plug in $W_{1}(t)$ and rearrange to arrive at an expression for the unknown function, $A_{1}(t)$.

$$
A_{1}(t)=\frac{c_{t} \Delta p_{w f}}{2 \sqrt{\pi t^{3}}}
$$

With this, the pressure drop at any point $(\tau, t)$ may be represented with 


$$
\Delta p(\tau, t)=\Delta p_{w f} \operatorname{erfc}\left(\frac{\tau}{\sqrt{4 t}}\right)
$$

The flux at any point $(\tau, t)$ may be calculated with the following.

$$
\begin{gathered}
q(\tau, t)=A_{1}(t) V_{1}(\tau, t) \\
q(\tau, t)=\Delta p_{w f} A \phi c_{t} \sqrt{\frac{\alpha}{\pi t}} e^{-\tau^{2} / 4 t}
\end{gathered}
$$

Set $\tau=0$ to obtain the expression for wellbore rate.

$$
\begin{gathered}
q_{w}=A_{1}(t) V_{1}(t) \\
q_{w}=\Delta p_{w f} A \phi c_{t} \sqrt{\frac{\alpha}{\pi t}}
\end{gathered}
$$

Alternatively, this may be written as shown below (in dimensional form), which is equivalent to the expression shown in Lee et al. (2003).

$$
\Delta p_{w f}=25.54 \frac{q_{w}}{A \phi c_{t}} \sqrt{\frac{t}{\alpha}}
$$

\section{2-Dimensional Solutions - Infinite Acting “Pillbox” Flow (IAPF)}

The process for deriving the 2-dimensional "pillbox" flow model is very similar to that of the IALF model. As with the linear flow case we present the process for deriving this solution with the asymptotic expansion method to set the stage for the development of the pillbox MTFW model. 
Consider a single infinite-conductivity planar fracture in an infinite homogeneous, isotropic medium (Figure 8). The fracture has height $h$ and is fullypenetrating. Since the fracture is infinitely-conductive, we consider it to be a planar source and thus give it negligible width. In this case the fracture will drain both perpendicular to its face and radially to its tips.

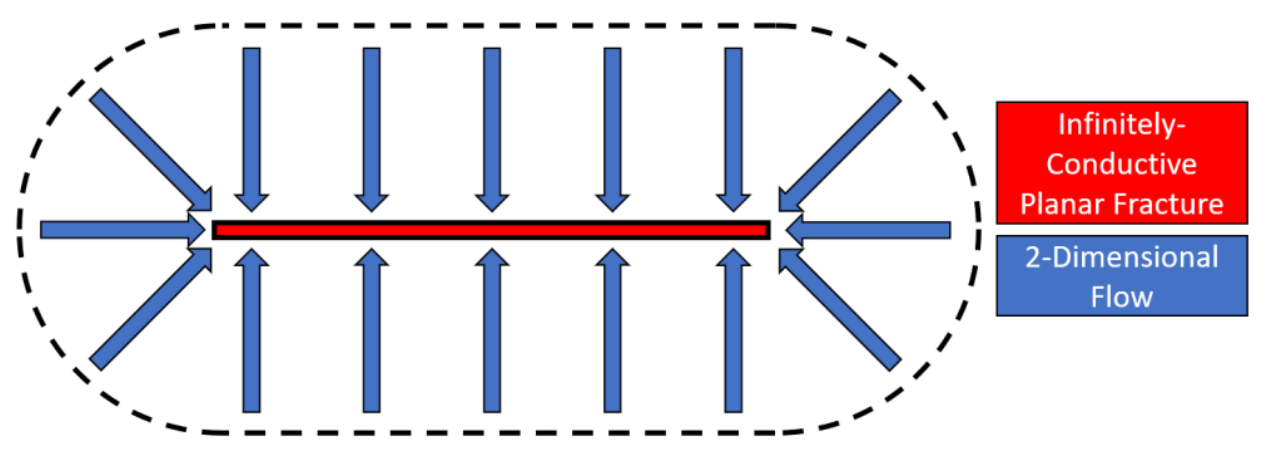

Figure 8 - Pillbox Model (after Malone et al. (2019)). Copyright 2019, SPE. Reproduced with permission of SPE. Further reproduction prohibited without permission.

As in the IALF case, to develop a diffusivity relationship we first define the $\tau$ parameter as the ratio of the shortest distance $r$ from any point in $(x, y)$ to the fracture (Figure 9). For a point located perpendicular to the fracture, this will simply be the perpendicular distance to the fracture. For a point beyond the fracture tips, this will be the radius of a semi-circle centered at the fracture tip that intersects that point.

$$
\tau=\frac{r}{\sqrt{\alpha}}
$$


We define pore volume next in terms of both $r$ and $\tau$

$$
\begin{gathered}
V_{p}(r)=\phi h\left(4 x_{f} r+\pi r^{2}\right) \\
V_{p}(\tau)=\phi h\left(4 x_{f} \tau \sqrt{\alpha}+\pi \tau^{2} \alpha\right)
\end{gathered}
$$

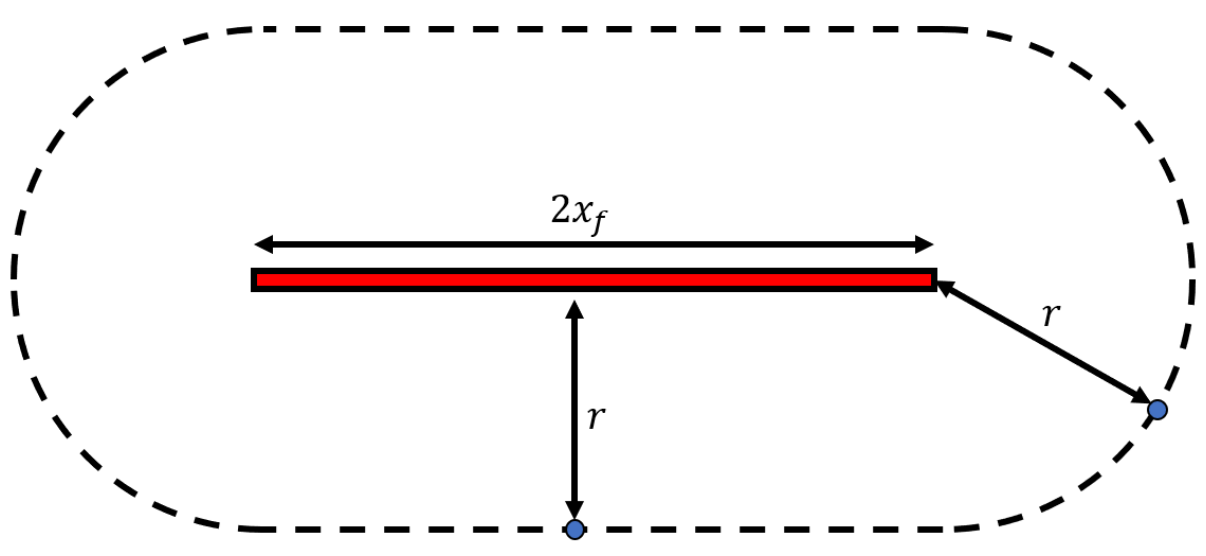

Figure 9 - Pillbox model distance parameters (from Malone et al. (2019)). Copyright 2019, SPE. Reproduced with permission of SPE. Further reproduction prohibited without permission.

Taking the derivative of pore volume with respect to $\tau$ we have

$$
w(\tau)=\frac{d V_{p}(\tau)}{d \tau}=\phi h\left(4 x_{f} \sqrt{\alpha}+2 \pi \tau \alpha\right)
$$

The volume and pressure drop moment integrals for $n=0,1$ come from (2.21) and (2.32)

$$
V_{n}(\tau, t)=\int_{\tau^{\prime}=\tau}^{\tau^{\prime}=\infty} w\left(\tau^{\prime}\right) \tau^{\prime n} e^{-\tau^{\prime 2} / 4 t} \partial \tau^{\prime}
$$




$$
W_{n}(\tau, t)=\int_{\tau^{\prime}=\tau}^{\tau^{\prime}=\infty} \frac{V_{n}\left(\tau^{\prime}\right)}{w\left(\tau^{\prime}\right)} \partial \tau^{\prime}
$$

In this case, an additional complication arises in that the pressure drop moment integrals cannot be evaluated analytically. Numerical integration will be required to evaluate the $W_{0}$ and $W_{1}$ terms.

$$
\begin{aligned}
& V_{0}(\tau, t)=4 h \sqrt{\pi \alpha t}\left\{x_{f} \phi \operatorname{erfc}\left(\frac{\tau}{2 \sqrt{t}}\right)+\sqrt{\pi \alpha t} e^{\frac{-\tau^{2}}{4 t}}\right\} \\
& V_{0}(t)=4 h \sqrt{\pi \alpha t}\left\{x_{f} \phi+\sqrt{\pi \alpha t}\right\} \\
& W_{0}(\tau, t)=2 \sqrt{\pi t} \int_{\tau^{\prime}=\tau}^{\tau^{\prime}=\infty} \frac{x_{f} \operatorname{erfc}\left(\frac{\tau^{\prime}}{2 \sqrt{t}}\right)+\sqrt{\pi \alpha t} e^{\frac{-\tau^{\prime 2}}{4 t}}}{2 x_{f}+\pi \sqrt{\alpha} \tau^{\prime}} d \tau^{\prime} \\
& W_{0}(t)=2 \sqrt{\pi t} \int_{\tau=0}^{\tau=\infty} \frac{x_{f} \operatorname{erfc}\left(\frac{\tau}{2 \sqrt{t}}\right)+\sqrt{\pi \alpha t} e^{\frac{-\tau^{2}}{4 t}}}{2 x_{f}+\pi \sqrt{\alpha} \tau} d \tau \\
& V_{1}(\tau, t)=4 \phi h\left[\left(2 x_{f} t \sqrt{\alpha}+\tau \pi t \alpha\right) e^{-\tau^{2} / 4 t}+\pi^{3 / 2} t^{3 / 2} \alpha \operatorname{erfc}\left(\frac{\tau}{2 \sqrt{t}}\right)\right] \\
& V_{1}(t)=4 \phi h\left[2 x_{f} t \sqrt{\alpha}+\pi^{3 / 2} t^{3 / 2} \alpha\right] \\
& W_{1}(\tau, t)=\int_{\tau^{\prime}=\tau}^{\tau^{\prime}=\infty} \frac{2\left[\left(2 x_{f} t \sqrt{\alpha}+\tau^{\prime} \pi t \alpha\right) e^{-\tau^{2} / 4 t}+\pi^{3 / 2} t^{3 / 2} \alpha \operatorname{erfc}\left(\frac{\tau^{\prime}}{2 \sqrt{t}}\right)\right]}{\left(2 x_{f} \sqrt{\alpha}+\pi \tau^{\prime} \alpha\right)} d \tau^{\prime}
\end{aligned}
$$




$$
W_{1}(t)=\int_{\tau=0}^{\tau=\infty} \frac{2\left[\left(2 x_{f} t \sqrt{\alpha}+\tau \pi t \alpha\right) e^{-\tau^{2} / 4 t}+\pi^{3 / 2} t^{3 / 2} \alpha \operatorname{erfc}\left(\frac{\tau}{2 \sqrt{t}}\right)\right]}{\left(2 x_{f} \sqrt{\alpha}+\pi \tau \alpha\right)} d \tau
$$

From here we can derive diffusivity expressions in the same way as for the IALF case.

To begin building the constant wellbore rate case, we start with (2.23) and say $n=0$.

$$
\begin{gathered}
q_{w}=A_{0}(t) V_{0}(t) \\
A_{0}=\frac{q_{w}}{4 h \sqrt{\pi \alpha t}\left\{x_{f} \phi+\sqrt{\pi \alpha t}\right\}}
\end{gathered}
$$

From here we can define the pressure drawdown expression with (2.34).

$$
\begin{gathered}
c_{t} \Delta p(\tau, t)=A_{0}(t) W_{0}(\tau, t) \\
\Delta p(\tau, t)=\frac{q_{w}}{4 c_{t} h \sqrt{\pi \alpha t}\left\{x_{f} \phi+\sqrt{\pi \alpha t}\right\}} \int_{\tau^{\prime}=\tau}^{\tau^{\prime}=\infty} \frac{x_{f} e r f c\left(\frac{\tau^{\prime}}{2 \sqrt{t}}\right)+\sqrt{\pi \alpha t} e^{\frac{-\tau^{\prime 2}}{4 t}}}{2 x_{f}+\pi \sqrt{\alpha} \tau^{\prime}} \partial \tau^{\prime}(2.86)
\end{gathered}
$$

For the constant-BHP case we begin with (2.34) and set $n=1$.

$$
c_{t} \Delta p(\tau, t)=A_{1}(t) W_{1}(\tau, t)
$$

With BHP constrained, we set $\tau=0$.

$$
c_{t} \Delta p_{w f}=A_{1}(t) W_{1}(t)
$$




$$
A_{1}(t)=\frac{\Delta p_{w f} c_{t}}{2 t}\left\{\int_{\tau=0}^{\tau=\infty} \frac{\left[\left(2 x_{f} t \sqrt{\alpha}+\tau \pi t \alpha\right) e^{-\tau^{2} / 4 t}+\pi^{3 / 2} t^{3 / 2} \alpha \operatorname{erfc}\left(\frac{\tau}{2 \sqrt{t}}\right)\right]}{\left(2 x_{f} \sqrt{\alpha}+\pi \tau \alpha\right)} d \tau\right\}^{-1}
$$

With that we express the pressure drawdown at any point $(\tau, t)$ as

$$
\Delta p(\tau, t)=\Delta p_{w f} \frac{\int_{\tau^{\prime}=\tau}^{\tau^{\prime}=\infty} \frac{\left[\left(2 x_{f} t \sqrt{\alpha}+\tau^{\prime} \pi t \alpha\right) e^{-\tau^{2} / 4 t}+\pi^{3 / 2} t^{3 / 2} \alpha \operatorname{erfc}\left(\frac{\tau^{\prime}}{2 \sqrt{t}}\right)\right]}{\left(2 x_{f} \sqrt{\alpha}+\pi \tau^{\prime} \alpha\right)} d \tau^{\prime}}{\int_{\tau=0}^{\tau=\infty} \frac{\left[\left(2 x_{f} t \sqrt{\alpha}+\tau \pi t \alpha\right) e^{-\tau^{2} / 4 t}+\pi^{3 / 2} t^{3 / 2} \alpha \operatorname{erfc}\left(\frac{\tau}{2 \sqrt{t}}\right)\right]}{\left(2 x_{f} \sqrt{\alpha}+\pi \tau \alpha\right)} d \tau}
$$

And wellbore flux may be expressed as

$$
\begin{gathered}
q_{w}=A_{1}(t) V_{1}(t) \\
q_{w}=\frac{4 \phi h \Delta p_{w f} c_{t}\left[x_{f} t \sqrt{\alpha}+\pi^{3 / 2} t^{3 / 2} \alpha\right]}{t}\left\{\int_{\tau=0}^{\tau=0} \frac{\left[\left(2 x_{f} t \sqrt{\alpha}+\tau \pi t \alpha\right) e^{-\tau^{2} / 4 t}+\pi^{3 / 2} t^{3 / 2} \alpha e r f c\left(\frac{\tau}{2 \sqrt{t}}\right)\right]}{\left(2 x_{f} \sqrt{\alpha}+\pi \tau \alpha\right)} d \tau\right\}
\end{gathered}
$$

These expressions are significantly more complex and must be evaluated numerically. In the cases presented in this study, we evaluate this integral using simple trapezoidal integration. 


\section{Development of MTFW Solutions}

In Section 2.3 we showed how the asymptotic approximation of the diffusivity equation may be used to derive a diffusivity formulation for a single fracture in either a 1-dimensional or 2-dimensional reservoir. In this section, we use the same methodology, in conjunction with the principle of superposition in space to derive a solution to the MTFW case, and account for the interactions between fractures during wellbore flow.

We have developed a set of solutions based on the asymptotic expansion to describe the flow behavior of a MTFW that can take fracture interference into account, thus enabling the distribution of flux to individual fractures and the calculation of a ratenormalized pressure curve for both the entire well and individual fractures. We developed a total of 8 solutions and compared their behavior to a reference model built in a commercial reservoir simulator. These 8 solutions include both 1-term and 2-term asymptotic approximations of both 1-dimensional (IALF) and 2-dimensional (IAPF) flow, for both constant wellbore rate and constant bottom hole pressure inner boundary conditions.

Take note that not every solution matches the commercial simulator response, and some solutions are not stable. We present them to document the issues we saw, and what degree of success should be expected for each implementation. 


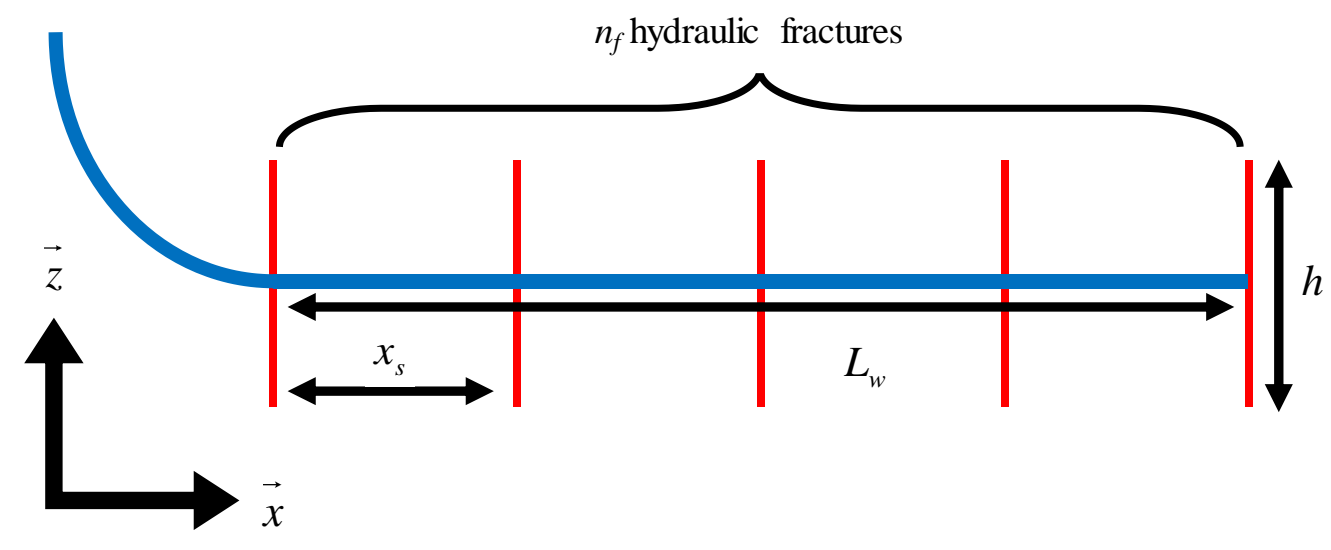

Figure 10 - MTFW System (X-Z Plane View)

Each solution considers an infinite, homogeneous, isotropic reservoir accessed by a horizontal well via a series of equally-spaced, infinitely-conductive, symmetrical, fully-penetrating planar fractures (Figure 10, Figure 11, Figure 12). We do not treat the well as fully-completed. It accesses the reservoir exclusively through the hydraulic fractures, so we may also describe this system as a series of interconnected planar sources. 


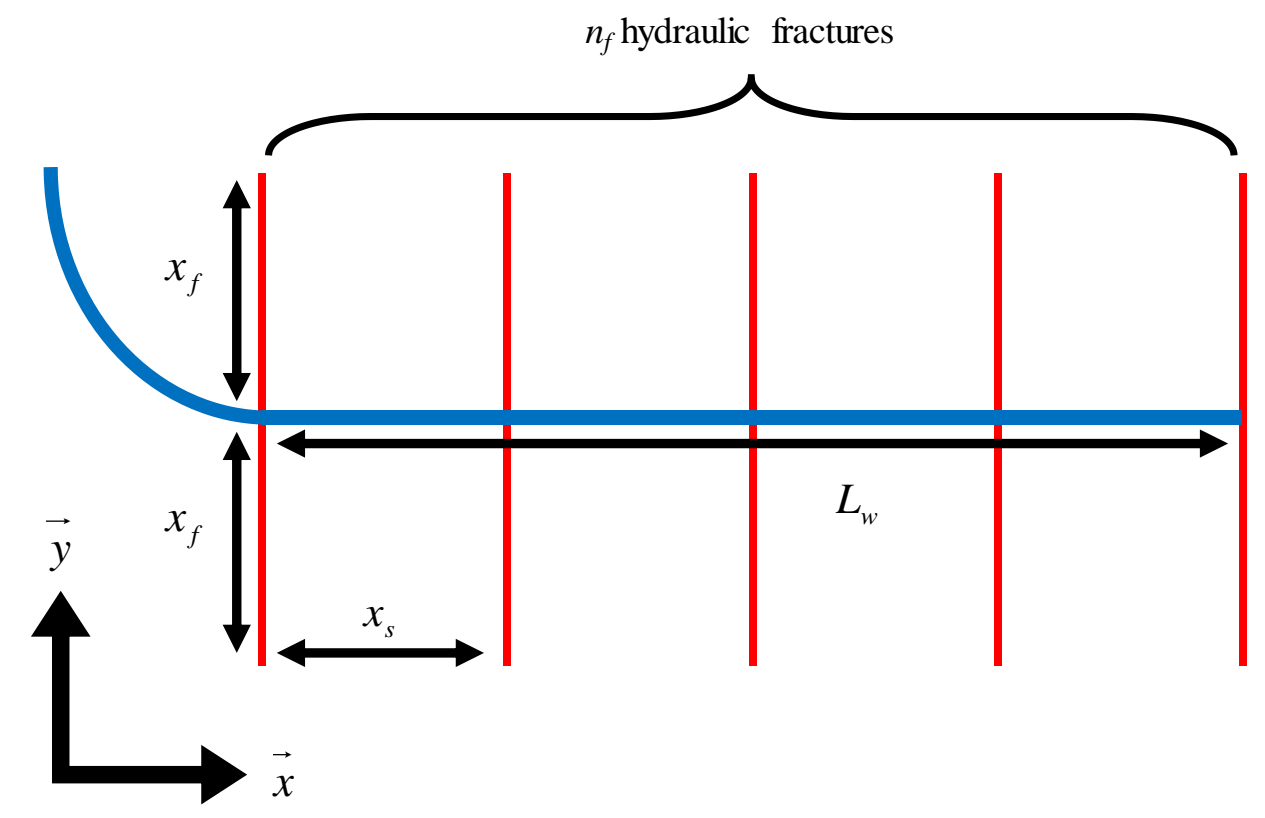

Figure 11 - MTFW System (X-Y Plane View)

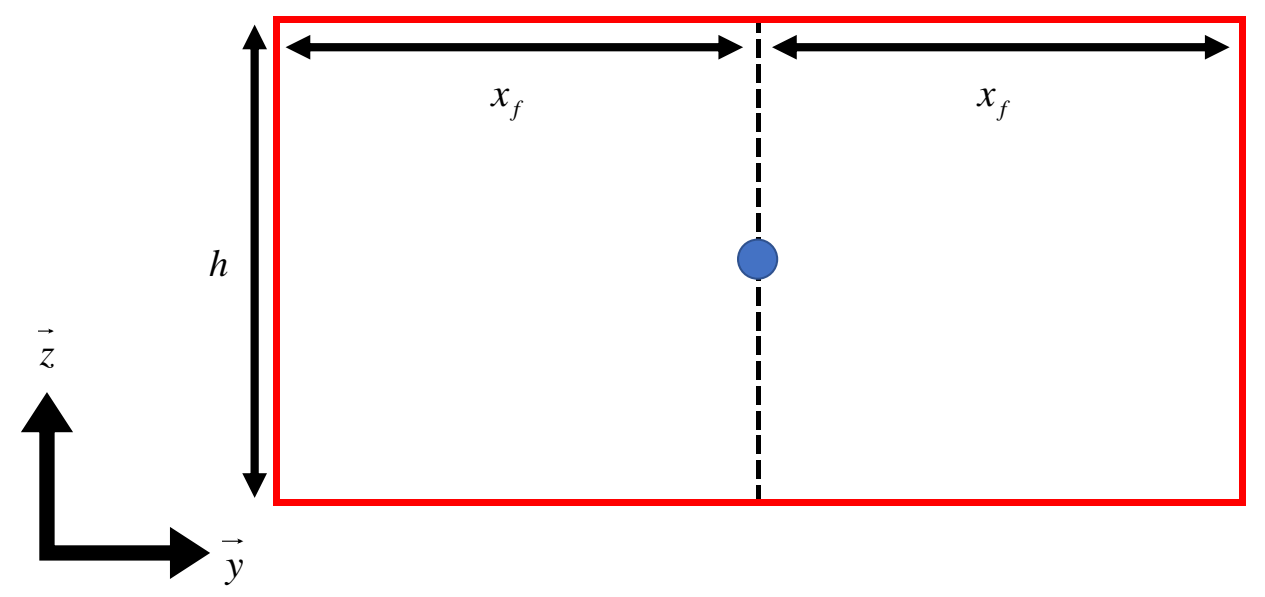

Figure 12 - MTFW System (Y-Z Plane View)

We evaluated both an infinite acting linear flow (IALF) system and an infinite acting pillbox flow (IAPF) system. In the IALF system we consider a well that sits in an 
infinite medium in the x-direction, and each fracture drains a growing box-shaped volume with a height equal to fracture height, a width equal to twice the fracture halflength and a length of infinity on both sides (Figure 13).

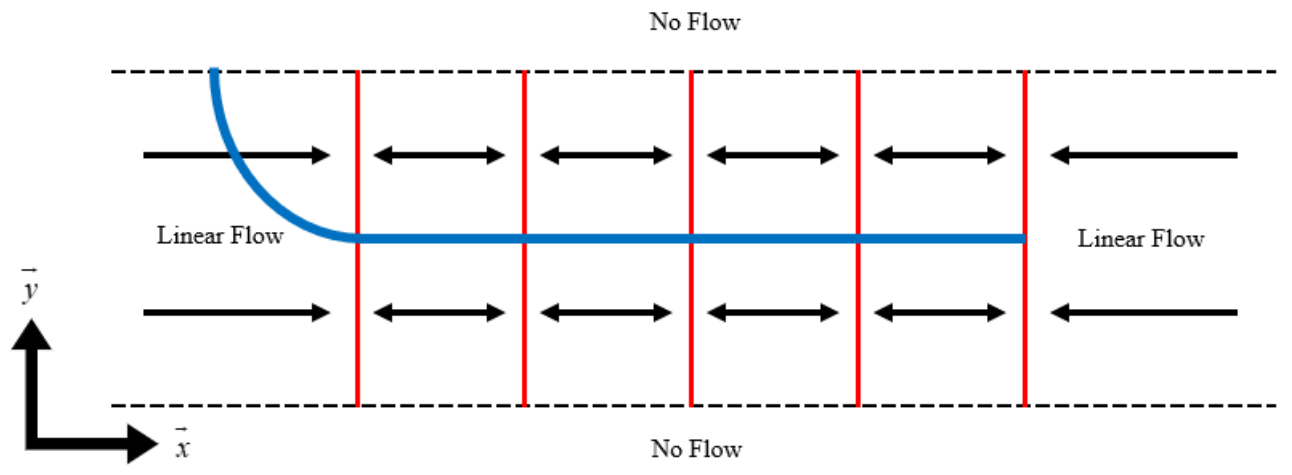

Figure 13 - IALF System Diagram

For the IAPF case, we consider a well that sits in an infinite medium in the X-Y plane with a height equal to fracture height. Each fracture drains a growing pillboxshaped volume as depicted in Figure 8, and the entire system appears as depicted in Figure 14. 


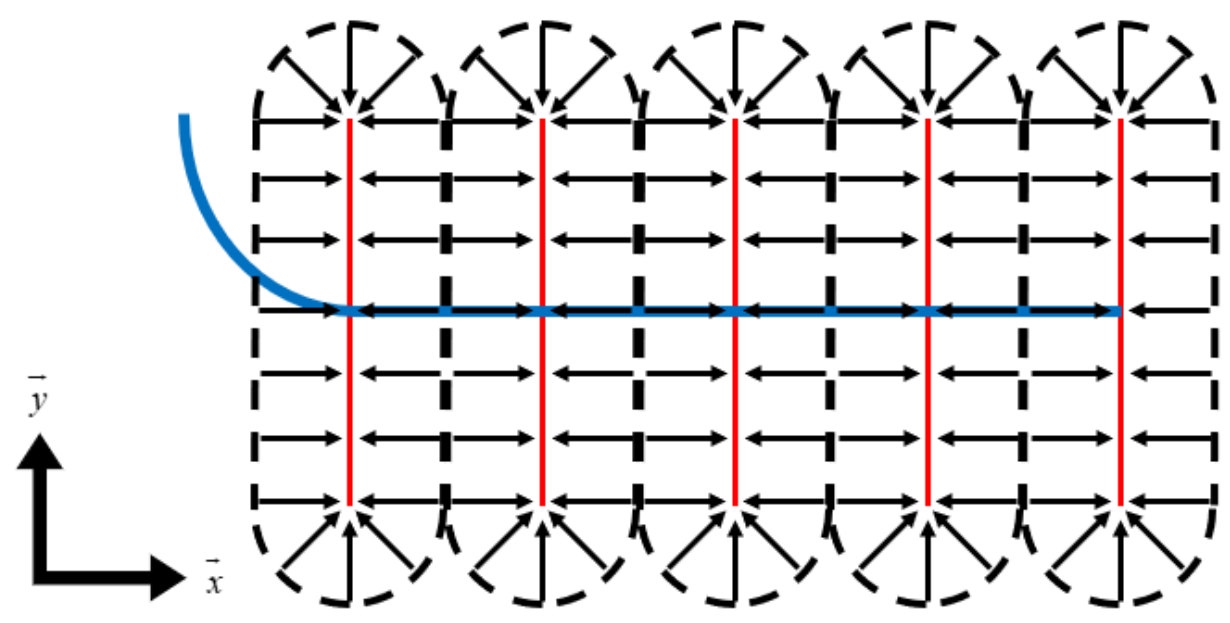

Figure 14 - IAPF System Diagram (after Malone et al. (2019)). Copyright 2019, SPE. Reproduced with permission of SPE. Further reproduction prohibited without permission.

\section{Variable Rate (1-term Ao Approximation)}

For the variable rate IBC, we constrain the well rate, $q_{w}$, and state that the well oil rate will be equal to the sum of the flux to each fracture $j$ out of the $n_{f}$ fractures connected to the wellbore. We say nothing of the derivative of wellbore rate or pressure, but assume that the $A_{0}(t)$ term alone can approximate $A_{0}+\tau A_{1}$ (Eq. (2.37)). We then define the wellbore flux as the sum of the flux from each fracture by (2.25).

$$
q_{w} \approx \sum_{j=1}^{n_{f}} A_{0, j}(t) V_{0}(t)
$$

We already assumed that both the fractures and the well are infinitely conductive. As a logical extension of this assumption, we state that the fractures are in complete hydraulic communication with each other and must be at the same pressure and 
experience the same pressure drawdown, which will equal the well's bottom hole flowing pressure drawdown, $\Delta p_{w f}$.

$$
\Delta p_{w f}=\Delta p_{w f, 1}=\Delta p_{w f, 2}=\ldots=\Delta p_{w f, n_{f}}
$$

With the principal of superposition, we say that the pressure drop seen at any individual fracture will equal the sum of the pressure drop induced by every fracture in the system. This will include both the fracture's self-induced pressure drawdown and the pressure drop induced by its neighbors. Again setting $n=0$ we base our total pressure drawdown expression on (2.34). We represent the total pressure drawdown at any one fracture $i$ as the sum of the pressure drawdown induced by all fractures $j=1 \ldots n_{f}$, where $\tau_{i j}$ is that value of $\tau$ for which $r$ equals the distance between fractures $i$ and $j$ (2.95).

$$
c_{t} \Delta p_{w f} \approx \sum_{j=1}^{n_{f}} A_{0, j}(t) W_{0}\left(\tau_{i j}, t\right)
$$

We now have a set of $n_{f}+1$ unknowns. There is an unknown function, $A_{0, j}$, for each fracture $\left(j=1 \ldots n_{f}\right)$, and an unknown shared pressure drop, $\Delta p_{w f}$. With the drawdown summation (2.95) we have $n_{f}$ equations, and with the flux equation (2.93) we have $n_{f}+1$. Represented as a system of equations, this becomes a simple matrix problem (2.96). The equation used to compute the terms $V_{0}(t)$ and $W_{0}(\tau, t)$ is dictated by the flow regime model. For an IALF calculation, we compute $V_{0}(t)$ with (2.47) and 
$W_{0}(\tau, t)$ with (2.48). For an IAPF calculation, we compute $V_{0}(t)$ with (2.76) and $W_{0}(\tau, t)$ with (2.77).

$\left[\begin{array}{ccccc}W_{0,1}\left(t, \tau_{11}\right) & W_{0,2}\left(t, \tau_{12}\right) & \ldots & W_{0, N}\left(t, \tau_{1 n_{f}}\right) & -1 \\ W_{0,1}\left(t, \tau_{21}\right) & W_{0,2}\left(t, \tau_{22}\right) & \ldots & \ldots & -1 \\ \ldots & \ldots & \ldots & \ldots & \ldots \\ W_{0,1}\left(t, \tau_{n_{f} 1}\right) & \ldots & \ldots & W_{0, N}\left(t, \tau_{n_{f} n_{f}}\right) & -1 \\ V_{0}(t) & V_{0}(t) & \ldots & V_{0}(t) & 0\end{array}\right]\left[\begin{array}{c}A_{0,1} \\ A_{0,2} \\ \ldots \\ A_{0, n_{f}} \\ c_{t} \Delta p_{w f}\end{array}\right]=\left[\begin{array}{c}0 \\ 0 \\ \ldots \\ 0 \\ q_{w}\end{array}\right]$

To solve, we simply consider this system instead as

$$
A \vec{x}=b
$$

and we may solve for the $A_{0}$ terms using matrix inversion as in (2.98).

$$
\vec{x}=A \backslash b
$$

Individual fracture flowrates are given by

$$
q_{j}(t)=A_{0, j}(t) V_{0}(t)
$$

And for individual fracture diagnostics, we use the rate-normalized pressure (RNP) as in $(2.100)$

$$
R N P_{j}(t)=\frac{q_{j}(t)}{\Delta p_{w f}(t)}
$$

For whole-well diagnostics, instead we use

$$
R N P_{w e l l}(t)=\frac{q_{w}}{\Delta p_{w f}(t)}
$$




\section{Constant Rate (2-term $A_{0}$ and $A_{1}$ Approximation)}

In this case we constrain the well rate, $q_{w}$, and state that the well oil rate will be equal to the sum of the flux to each fracture $j$ out of the $n_{f}$ fractures connected to the wellbore. We also constrain the derivative of the well rate with respect to time, $\frac{\partial q_{w}}{\partial t}$, to zero, and set the well's derivative as equal the sum of the rate derivates of all fractures. With this, the individual fracture fluxes are permitted to vary, but the total well rate is constrained. The derivative constraint (2.103) comes from (2.35).

$$
\begin{gathered}
q_{w} \approx \sum_{j=1}^{n_{f}} \sum_{n=0}^{1} A_{n, j}(t) V_{n}(t) \\
\frac{1}{w(0)} \frac{\partial q_{w}}{\partial t}=-\sum_{j=1}^{n_{f}} A_{1, j}=0
\end{gathered}
$$

We then constrain pressure in the same way as in the variable rate case - we state that the fractures are in complete hydraulic communication with each other and must be at the same pressure and experience the same pressure drawdown, which will equal the well's bottom hole flowing pressure drawdown, $\Delta p_{w f}$. The same also applies to the derivative of the pressure drop with respect to time.

$$
\begin{gathered}
\Delta p_{w f}=\Delta p_{w f, 1}=\Delta p_{w f, 2}=\ldots=\Delta p_{w f, n_{f}} \\
\frac{\partial \Delta p_{w f}}{\partial t}=\frac{\partial \Delta p_{w f, 1}}{\partial t}=\frac{\partial \Delta p_{w f, 2}}{\partial t}=\ldots=\frac{\partial \Delta p_{w f, n_{f}}}{\partial t}
\end{gathered}
$$


Once again using the principle of superposition, we say that the pressure drop seen at any individual fracture will equal the sum of the pressure drop induced by every fracture in the system, including itself. In this case we set $n=1$ and base our pressure drawdown expression on Eq. (2.34). We represent the total pressure drawdown at any one fracture $i$ as the sum of the pressure drawdown induced by all fractures $j=1 \ldots n_{f}$, where $\tau_{i j}$ is that value of $\tau$ for which $r$ equals the distance between fractures $i$ and $j$ (Eq. (2.95)).

$$
c_{t} \Delta p_{w f} \approx \sum_{j=1}^{n_{f}} \sum_{n=0}^{1} A_{n, j} W_{n}\left(\tau_{i j}, t\right)
$$

For our final set of expressions, we use Eq. (2.16) to represent the total pressure drawdown derivative with respect to time at any one fracture $i$ as the sum of the pressure drawdown derivatives induced by all fractures $j=1 \ldots n_{f}$, where $\tau_{i j}$ is that value of $\tau$ for which $r$ equals the distance between fractures $i$ and $j$ (Eq. (2.16)).

$$
c_{t} \frac{\partial \Delta p_{w f}}{\partial t} \approx \sum_{j=1}^{N}\left(A_{0, j}+A_{1, j} \tau_{i j}\right) e^{-\tau_{i j}^{2} / 4 t}
$$

We have two unknown functions for each fracture, $A_{0}$ and $A_{1}$, an unknown shared pressure drop, $\Delta p_{w f}$, an unknown shared pressure drop derivative, $\frac{\partial \Delta p_{w f}}{\partial t}$, giving us $\left(2 n_{f}+2\right)$ unknowns. Equation (2.102) gives us 1 equation, Equation (2.103) gives us 1 equation, Equation (2.106) gives us $n_{f}$ equations, and Equation (2.107) gives us $n_{f}$ 
equations - together giving us $\left(2 n_{f}+2\right)$ equations to solve. Represented as a matrix problem, we have Equation (2.108).

\begin{tabular}{|c|c|c|c|c|c|c|c|c|c|c|}
\hline 1 & 1 & $\ldots$ & 1 & 0 & 0 & $\ldots$ & 0 & 0 & 0 & $A_{1,1}$ \\
\hline$V_{1}(t)$ & $V_{1}(t)$ & $\ldots$ & $V_{1}(t)$ & $V_{0}(t)$ & $V_{0}(t)$ & $\ldots$ & $V_{0}(t)$ & 0 & 0 & $A_{1,2}$ \\
\hline$W_{1}(t)$ & $W_{1}\left(t, \tau_{21}\right)$ & $\cdots$ & $W_{1}\left(t, \tau_{n_{f 1}}\right)$ & $W_{0}(t)$ & $W_{0}\left(t, \tau_{21}\right)$ & $\ldots$ & $W_{0}\left(t, \tau_{n_{f} 1}\right)$ & 1 & 0 & $\cdots$ \\
\hline$W_{1}\left(t, \tau_{12}\right)$ & $W_{1}(t)$ & $W_{1}\left(t, \tau_{32}\right)$ & $\ldots$ & $W_{0}\left(t, \tau_{12}\right)$ & $W_{0}(t)$ & $W_{0}\left(t, \tau_{32}\right)$ & $\ldots$ & 1 & 0 & $A_{1, n_{f}}$ \\
\hline$\cdots$ & $W_{1}\left(t, \tau_{23}\right)$ & $\ldots$ & $\ldots$ & $\cdots$ & $W_{0}\left(t, \tau_{23}\right)$ & $\ldots$ & $\ldots$ & $\cdots$ & $\cdots$ & $A_{0,1}$ \\
\hline$W_{1}\left(t, \tau_{1 n_{f}}\right)$ & $\ldots$ & $\ldots$ & $W_{1}(t)$ & $W_{0}\left(t, \tau_{1 n_{f}}\right)$ & $\ldots$ & $\ldots$ & $W_{0}(t)$ & 1 & 0 & $A_{0,2}$ \\
\hline 0 & $\tau_{21} e^{-\tau_{21}^{2} / 4 t}$ & $\ldots$ & $\tau_{n_{f} 1} e^{-\tau_{\tau_{f}}^{2} / / t}$ & 1 & $e^{-\tau_{2}^{2} / 4 t}$ & $\ldots$ & $e^{-\tau_{n, f}^{2} / 4 t}$ & 0 & 1 & $\ldots$ \\
\hline$\tau_{12} e^{-\tau_{12}^{2} / 4 t}$ & 0 & $\tau_{32} e^{-\tau_{32}^{2} / 4 t}$ & $\ldots$ & $e^{-\tau_{1 / 2}^{2} / 4 t}$ & 1 & $e^{-\tau_{32}^{2} / 4 t}$ & $\ldots$ & 0 & 1 & $A_{0, n_{f}}$ \\
\hline$\ldots$ & $\tau_{23} e^{-\tau_{23}^{2} / 4 t}$ & $\ldots$ & $\cdots$ & $\ldots$ & $e^{-\tau_{23}^{2} / 4 t}$ & $\ldots$ & $\ldots$ & $\cdots$ & $\ldots$ & $c_{t} \Delta p_{w j}$ \\
\hline$\tau_{1 n_{f}} e^{-\tau_{n_{j}} / / 4 t}$ & $\cdots$ & $\cdots$ & 0 & $e^{-\tau_{l_{h} / / 4 t}}$ & $\ldots$ & $\ldots$ & 1 & 0 & 1 & \\
\hline
\end{tabular}

We consider this as the following

$$
A \vec{x}=b
$$

And solve with matrix inversion

$$
\vec{x}=A \backslash b
$$

Individual fracture flow rates may be computed with

$$
q_{j}(t) \approx \sum_{n=0}^{1} A_{n, j}(t) V_{n}(t)
$$

For individual fracture diagnostics, we can use RNP

$$
R N P_{j}(t)=\frac{q_{j}(t)}{\Delta p_{w f}(t)}
$$

And for whole-well diagnostics, we use the whole-well RNP 


$$
R N P_{w e l l}(t)=\frac{q_{w}}{\Delta p_{w f}(t)}
$$

\section{Constant BHP (1-term A Approximation)}

This case represents the non-functional IBC referenced by Eq. (2.38). This case has been tested to have a complete set of solutions but does not provide a sufficient estimate of wellbore flow or pressure relative to a reference model. For this case we constrain neither the wellbore flow or bottom hole pressure's time derivatives, and instead assume that the $\tau A_{1}(t)$ term alone can sufficiently approximate $A_{0}+\tau A_{1}$. As with the constant-rate cases, we state that the fractures are in complete hydraulic communication with each other and must be at the same pressure and experience the same pressure drawdown, which will equal the well's bottom hole flowing pressure drawdown, $\Delta p_{w f}$.

$$
\Delta p_{w f}=\Delta p_{w f, 1}=\Delta p_{w f, 2}=\ldots=\Delta p_{w f, n_{f}}
$$

Using the principle of superposition, we represent the total pressure drawdown at any one fracture $i$ as the sum of the pressure drawdown induced by all fractures $j=1 \ldots n_{f}$, where $\tau_{i j}$ is that value of $\tau$ for which $r$ equals the distance between fractures $i$ and $j$ (2.95). We acknowledge that the principle of superposition is not applicable to constant-BHP boundary conditions and present this methodology simply to document its results. 


$$
c_{t} \Delta p_{w f}=\sum_{j=1}^{n_{f}} A_{1}(t) W_{1}\left(\tau_{i j}, t\right)
$$

The well rate will equal the sum of the rate from all individual fractures. In this case the well rate is not constrained.

$$
q_{w} \approx \sum_{j=1}^{n_{f}} A_{1, j}(t) V_{1}(t)
$$

We have a total of $n_{f}+1$ unknowns $-n_{f}$ unknown function $\left(A_{1}\right)$ terms and a single unknown well rate. Written as a matrix problem, this system of equations becomes the following.

$$
\left[\begin{array}{ccccc}
W_{1,1}\left(t, \tau_{11}\right) & W_{1,2}\left(t, \tau_{12}\right) & \ldots & W_{1, N}\left(t, \tau_{1 n_{f}}\right) & 0 \\
W_{1,1}\left(t, \tau_{21}\right) & W_{1,2}\left(t, \tau_{22}\right) & \ldots & \ldots & 0 \\
\ldots & \ldots & \ldots & \ldots & \ldots \\
W_{1,1}\left(t, \tau_{n_{f} 1}\right) & \ldots & \ldots & W_{1, N}\left(t, \tau_{n_{f} n_{f}}\right) & 0 \\
V_{1}(t) & V_{1}(t) & \ldots & V_{1}(t) & -1
\end{array}\right]\left[\begin{array}{c}
A_{1,1} \\
A_{1,2} \\
\ldots \\
A_{1, n_{f}} \\
q_{w}
\end{array}\right]=\left[\begin{array}{c}
c_{t} \Delta p_{w f} \\
c_{t} \Delta p_{w f} \\
\ldots \\
c_{t} \Delta p_{w f} \\
0
\end{array}\right]
$$

We consider this as the following

$$
A \vec{x}=b
$$

And solve with matrix inversion

$$
\vec{x}=A \backslash b
$$

Individual fracture flow rates may be computed with

$$
q_{j}(t) \approx A_{1, j}(t) V_{1}(t)
$$


For individual fracture diagnostics, we can use RNP

$$
R N P_{j}(t)=\frac{q_{j}(t)}{\Delta p_{w f}(t)}
$$

And for whole-well diagnostics, we use the whole-well RNP

$$
R N P_{w e l l}(t)=\frac{q_{w}}{\Delta p_{w f}(t)}
$$

\section{Constant BHP (2-term $A_{0}$ and $A_{1}$ Approximation)}

Here we constrain the bottom hole pressure, $\Delta p_{w f}$, and its derivative with respect to

time, $\frac{\partial \Delta p_{w f}}{\partial t}(2.124)$. We state that the fractures are in complete hydraulic communication with each other and must be at the same pressure and experience the same pressure drawdown, which will equal the well's bottom hole flowing pressure drawdown, $\Delta p_{w f}$.

$$
\begin{gathered}
\Delta p_{w f}=\Delta p_{w f, 1}=\Delta p_{w f, 2}=\ldots=\Delta p_{w f, n_{f}} \\
c_{t} \frac{\partial \Delta p_{w f}}{\partial t} \approx \sum_{j=1}^{N}\left(A_{0, j}+A_{1, j} \tau_{i j}\right) e^{-\tau_{i j}^{2} / 4 t}=0
\end{gathered}
$$

Using the principle of superposition, we represent the total pressure drawdown at any one fracture $i$ as the sum of the pressure drawdown induced by all fractures $j=1 \ldots n_{f}$, where $\tau_{i j}$ is that value of $\tau$ for which $r$ equals the distance between fractures $i$ and $j$ (2.95). We acknowledge that the principle of superposition is not applicable to 
constant-BHP boundary conditions and present this methodology simply to document its results.

$$
c_{t} \Delta p_{w f} \approx \sum_{j=1}^{n_{f}} \sum_{n=0}^{1} A_{n, j} W_{n}\left(\tau_{i j}, t\right)
$$

With this we have a system of $2 n_{f}$ unknown functions, $A_{0}$ and $A_{1}$ for each fractures, with $2 n_{f}$ equations between (2.124) and (2.125). In matrix form, we have Eq. (2.126).

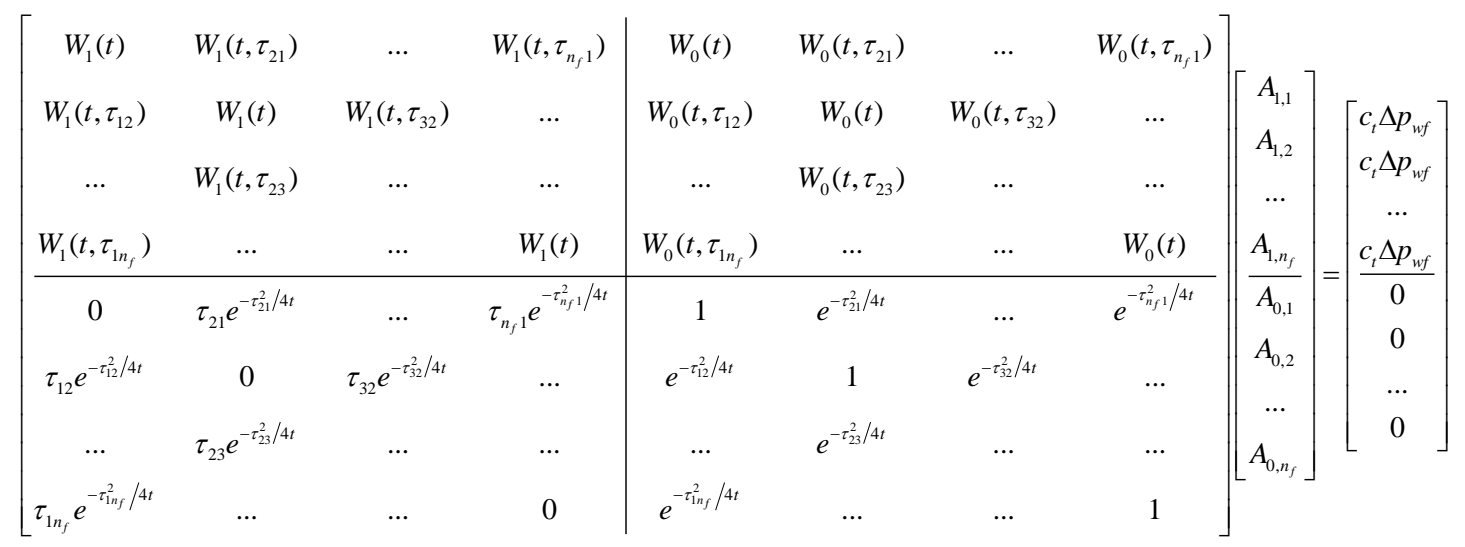

We consider this as the following

$$
A \vec{x}=b
$$

And solve with matrix inversion

$$
\vec{x}=A \backslash b
$$

Individual fracture flow rates may be computed with

$$
q_{j}(t) \approx \sum_{n=0}^{1} A_{n, j}(t) V_{n}(t)
$$


For individual fracture diagnostics, we can use RNP

$$
R N P_{j}(t)=\frac{q_{j}(t)}{\Delta p_{w f}(t)}
$$

And for whole-well diagnostics, we use the whole-well RNP

$$
R N P_{\text {well }}(t)=\frac{q_{w}}{\Delta p_{w f}(t)}
$$




\section{MTFW Solution Testing \& Results Summary \\ Reference Models}

We constructed two reference models in the reservoir commercial simulator, Eclipse. Both models incorporated a horizontal well intersecting 20 planar fractures with infinite conductivity. Fractures were represented explicitly as grid blocks with a width of $0.05 \mathrm{ft}$. A tartan simulation grid was used, with grid block widths increasing logarithmically with perpendicular distance from the fracture blocks. Well completions were simply modeled as zero-skin perforations in the fracture cells. Reservoir properties were kept consistent between models and shown in Table 1. Both a constant well rate ( $q=0.1 b b l / d a y)$ and constant $\operatorname{BHP}\left(\Delta p_{w f}=3000 p s i\right)$ inner boundary condition were tested.

For comparison with the IALF analytic solutions, we used a fully linear reservoir model, constructed as a channel with a width and height equal to that of the fractures.

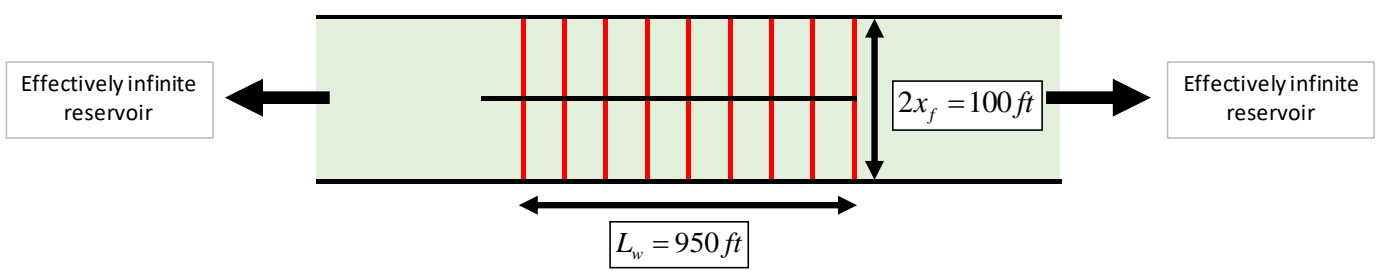

Figure 15 - IALF Reference Model Dimensions 
Though technically a 3-dimensional model, this reservoir model is effectively 1dimensional. The reservoir is bounded beyond the tips of the fractures, but effectively infinite in both directions perpendicular to the fracture faces (Figure 15).

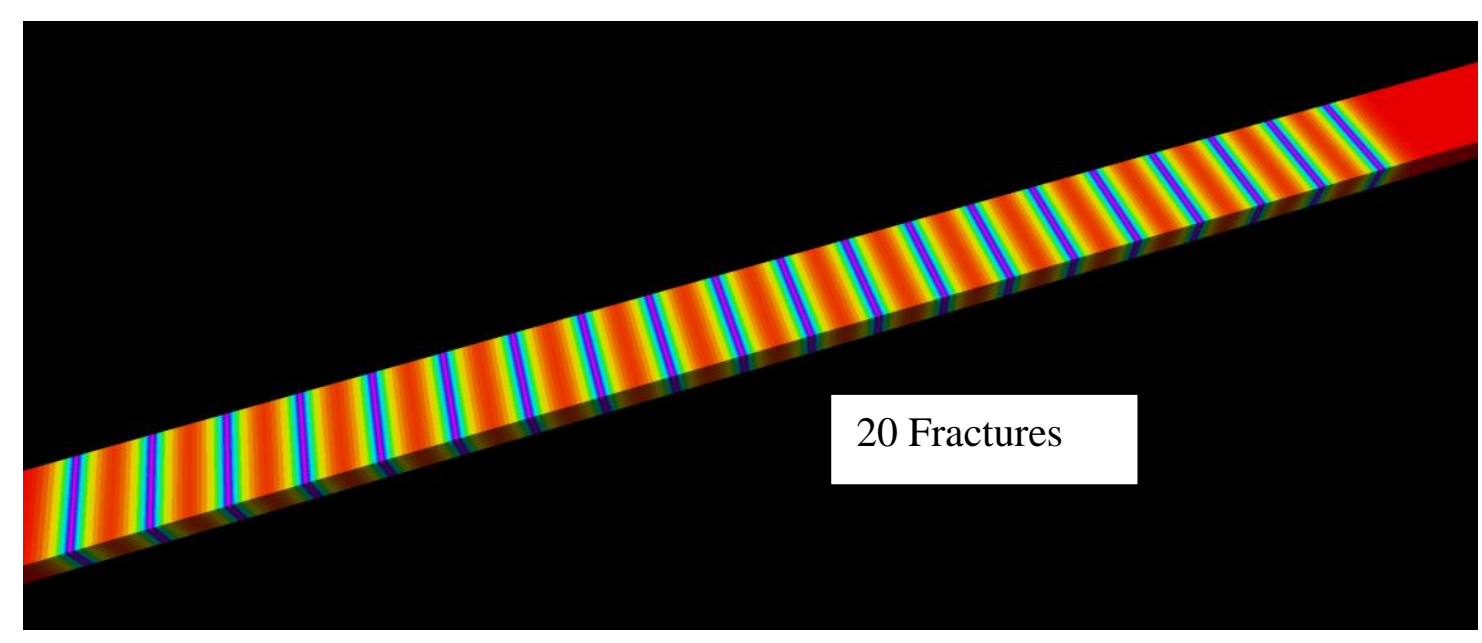

Figure 16 - IALF Reference Model Screenshot (Pressure Property Displayed) 


\begin{tabular}{|rcl|}
\hline Reference Model Properties \\
\hline Rock \& Fluid Properties & \\
\hline Permeability, $\mathrm{k}=$ & $\mathbf{0 . 0 0 0 2}$ & $\mathrm{md}$ \\
Porosity, $\phi=$ & $\mathbf{6 \%}$ & \\
Total Compressibility, $\mathrm{c}_{\mathrm{t}}=$ & $\mathbf{2 * 1 0 ^ { - 5 }}$ & $\mathrm{psi}^{-1}$ \\
Oil Viscosity, $\mu=$ & $\mathbf{1}$ & $\mathrm{cp}$ \\
Pay Height, $\mathrm{h}=$ & $\mathbf{3 0}$ & $\mathrm{ft}$ \\
Formation Volume Factor, $\mathrm{B}=$ & $\mathbf{1}$ & $\mathrm{rb} / \mathrm{stb}$ \\
Diffusivity, $\alpha=$ & $\mathbf{0 . 0 4 4}$ & $\mathrm{ft} / \mathrm{hr}$ \\
\hline Model Fracture Properties & \\
\hline Fracture Half-Length, $\mathrm{x}_{\mathrm{f}}=$ & $\mathbf{5 0}$ & $\mathrm{ft}$ \\
Fracture Spacing, $\mathrm{x}_{\mathrm{s}}=$ & $\mathbf{5 0}$ & $\mathrm{ft}$ \\
Number of Fractures, $\mathrm{N}_{\mathrm{f}}=$ & $\mathbf{2 0}$ & $\mathrm{fractures}$ \\
Fracture Permeability, $\mathrm{k}_{\mathrm{f}}=$ & $\mathbf{1 0 0 0}$ & $\mathrm{md}$ \\
Fracture Width, $\mathrm{w}_{\mathrm{f}}=$ & $\mathbf{0 . 0 5}$ & $\mathrm{ft}$ \\
Dimensionless Frac. Cond., $\mathrm{C}_{\mathrm{rd}}=$ & $\mathbf{1 6 0 0}$ & (infinite Cond.) \\
\hline
\end{tabular}

Table 1 - Reference Model Reservoir Properties (from Malone et al. (2019)). Copyright 2019, SPE. Reproduced with permission of SPE. Further reproduction prohibited without permission.

For comparison with the IAPF analytic solutions, we used a bounded reservoir model, wherein the horizontal well sits in the middle of a bounded rectangular reservoir. In this case the reservoir thickness also equals the fracture height. We did not make any attempts to represent an infinite reservoir in this case, simply to save time on simulation runs. This will cause the numerical simulation results to differ slightly from the analytic solutions during very late time. 


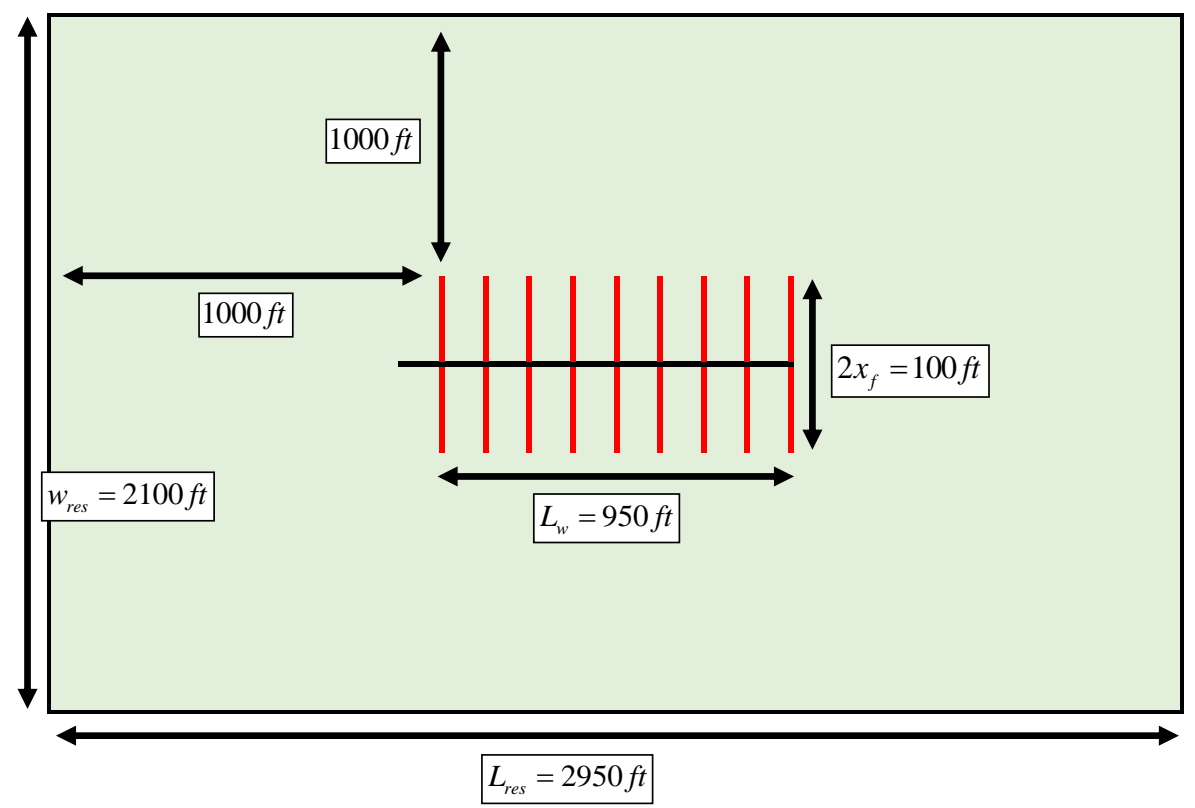

Figure 17 - IAPF Reference Model Dimensions (from Malone et al. (2019)). Copyright 2019, SPE. Reproduced with permission of SPE. Further reproduction prohibited without permission.

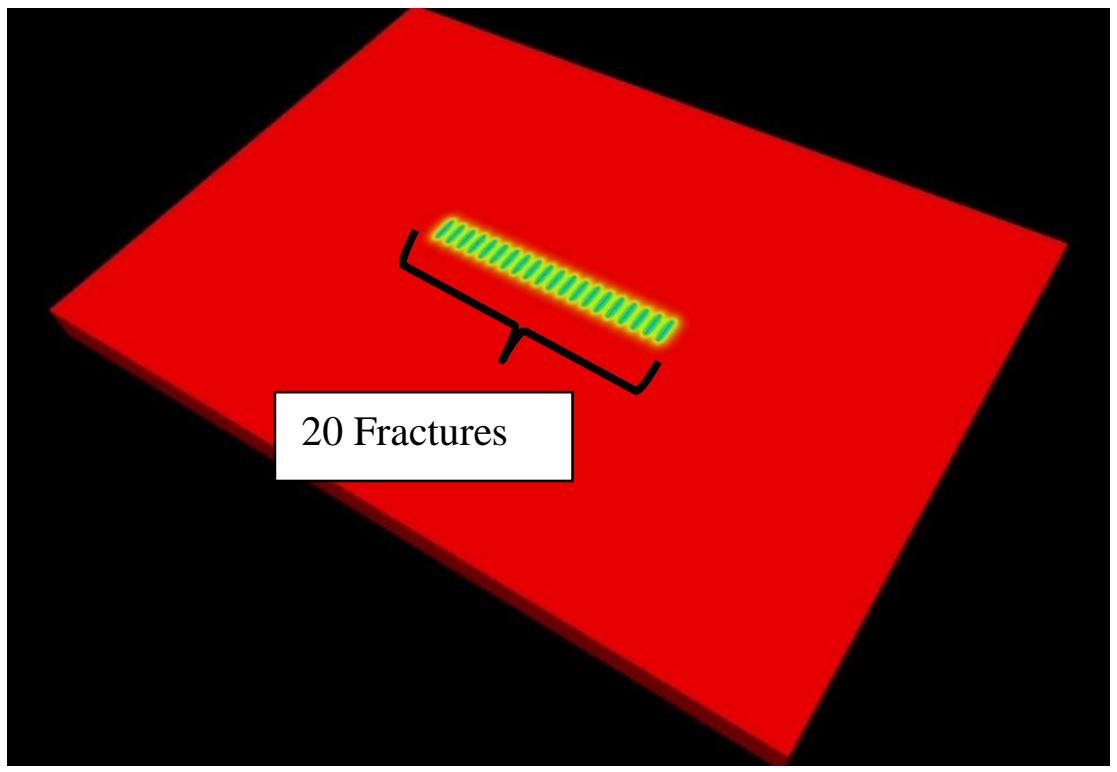

Figure 18 - IAPF Reference Model Screenshot (Pressure Property Displayed) 
With constant rate boundary conditions, the simulations show the typical expected progression of flow regimes for this well and reservoir geometry. Note that in the following descriptions we use the rate-normalized pressure's derivative with respect to the natural logarithm of time $\left(R N P^{\prime}\right)$ as a flow regime identifier. For the linear reservoir case (Figure 19) we see an initial linear flow period (shown by a half-slope), then a fracture interference period (nearly unit slope), and then a transition to a fully linear flow out to infinite time.

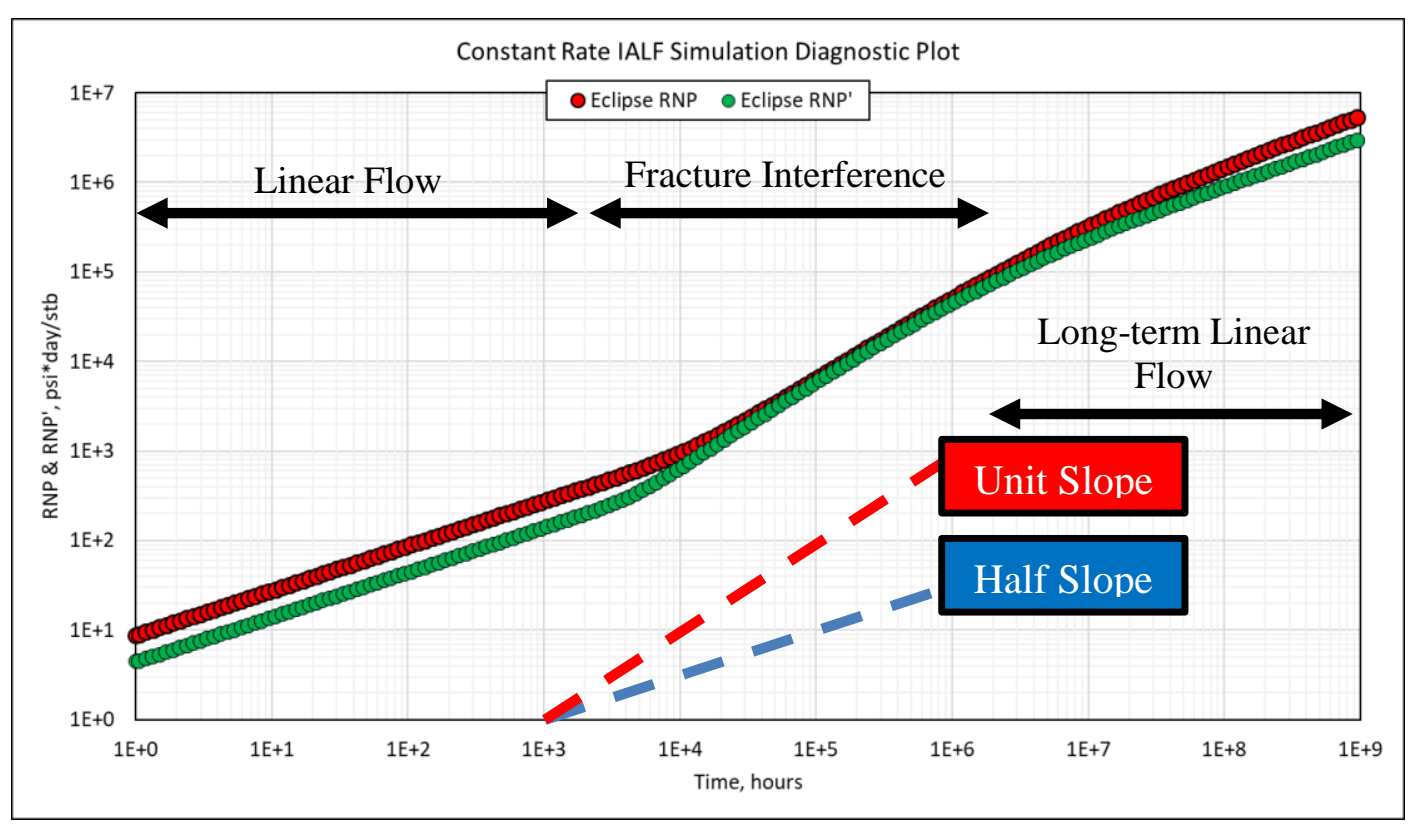

Figure 19 - IALF Constant-Rate Simulation Diagnostic Plot 
For the bounded 2-dimensional reservoir case (Figure 20) we see an initial linear flow period (shown by a half-slope). This is followed by a very brief transition to elliptical flow, shown as a very small dip from the linear half-slope (zoomed in view in Figure 21). Fracture interference then appears as a near unit slope. Next, we see the transition to radial flow as the fractures act as a single drainage source (trend towards a zero-slope), and then a pseudo steady state signature (unit slope) as the reservoir boundary effects begin.

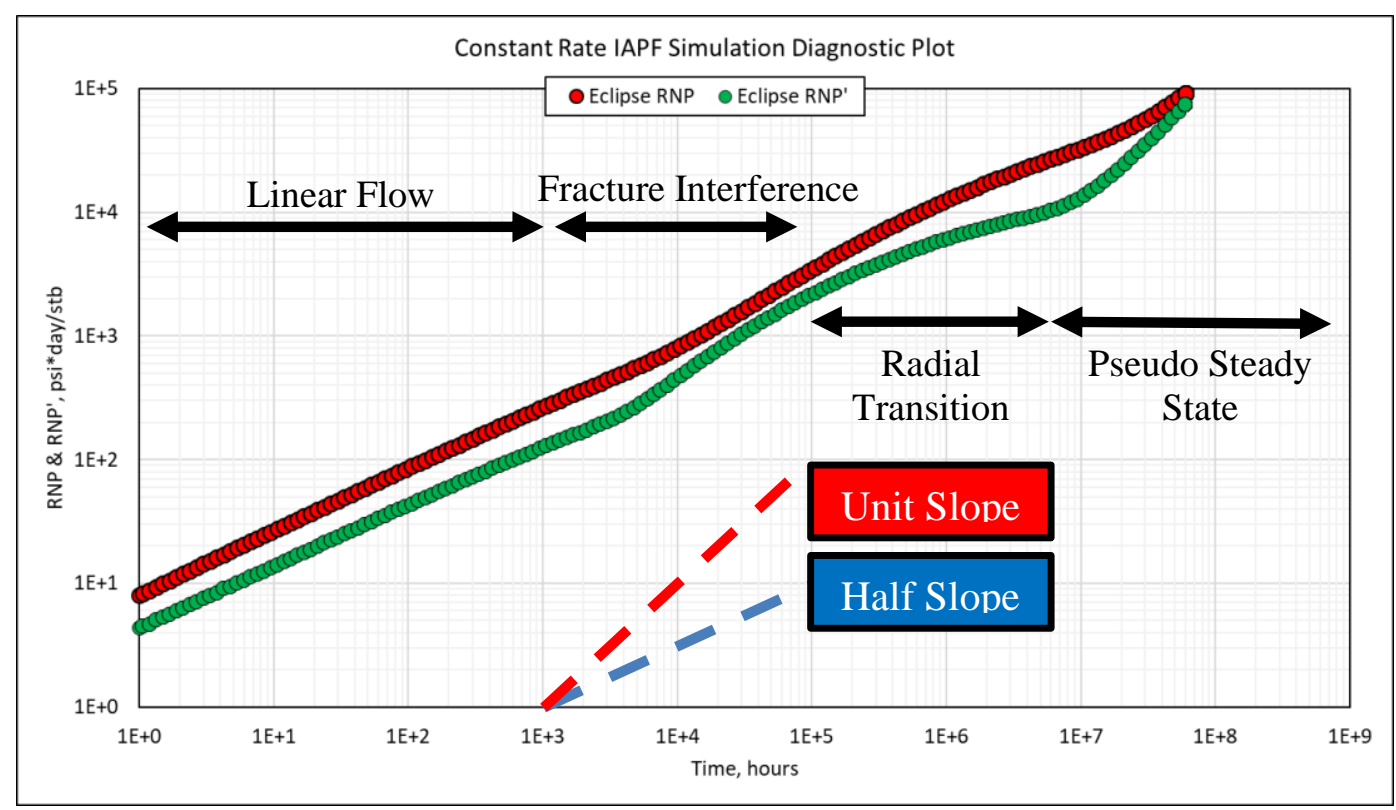

Figure 20 - IAPF Constant-Rate Simulation Diagnostic Plot (after Malone et al. (2019)). Copyright 2019, SPE. Reproduced with permission of SPE. Further reproduction prohibited without permission. 


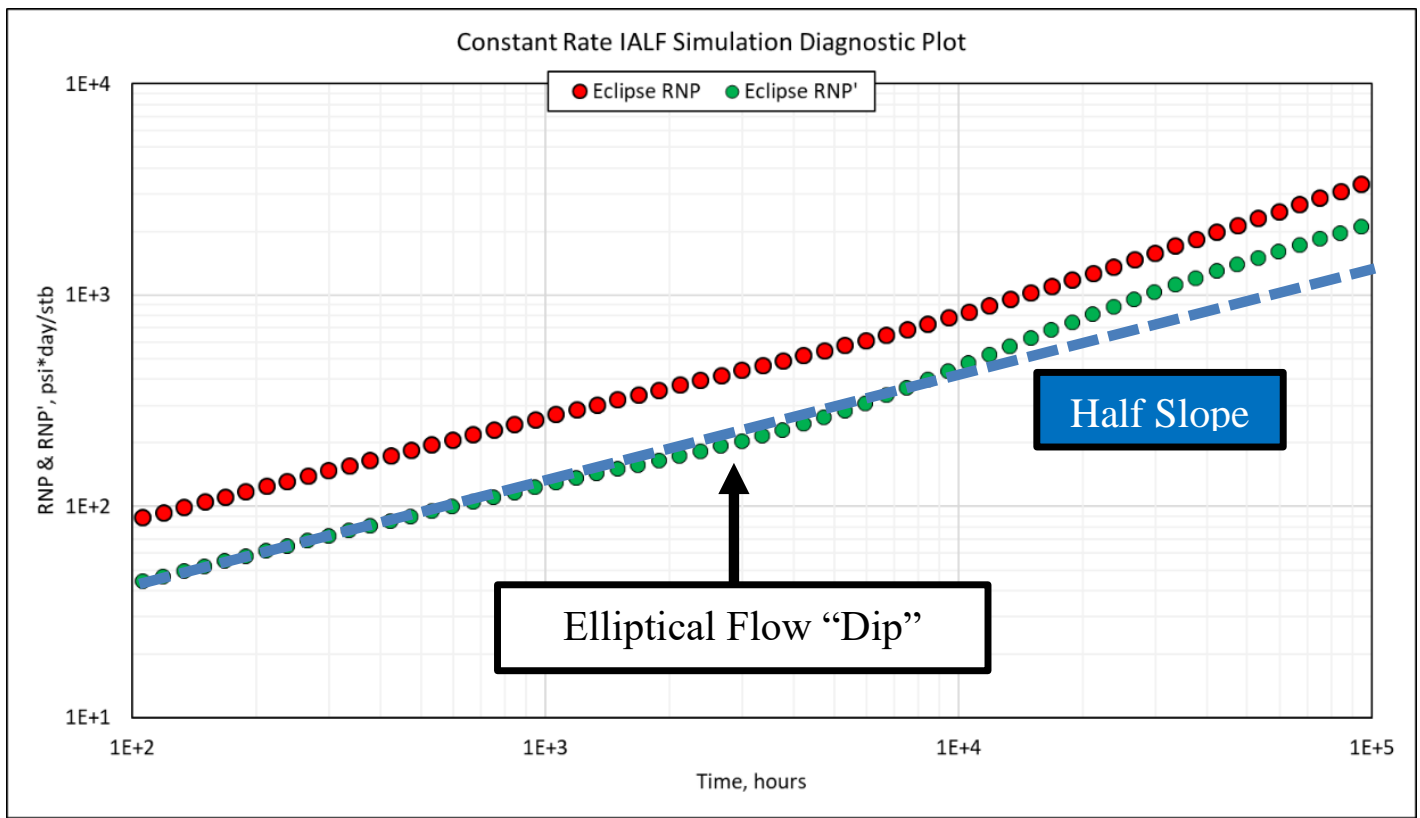

Figure 21 - IAPF Constant-Rate Simulation - Elliptical Flow Dip Signature (zoomed in view) (after Malone et al. (2019)). Copyright 2019, SPE. Reproduced with permission of SPE. Further reproduction prohibited without permission.

Interpreting diagnostic plots of RNP and RNP' versus time can be difficult for production data under a constant-BHP inner boundary condition. We can ease this interpretation through use of material balance time $\left(t_{m b}\right)$ (Equation (2.132)), which is the superposition function for pseudo-steady state flow. By plotting RNP and RNP' versus $t_{m b}$ we can generate a diagnostic plot from constant-BHP or varying-BHP, varying-rate data that will appear like a constant-rate diagnostic plot (Palacio and Blasingame 1993; Song and Ehlig-Economides 2011). Note that when we do this, RNP' must also be computed as a derivative with respect to the natural logarithm of $t_{m b}$ instead of time (2.133), and will be denoted as $R N P^{\prime}\left(t_{m b}\right)$. We plot the constant-BHP reference 
simulation results here as functions of time (which will be used as the reference to our analytic solutions), but also as functions of $t_{m b}$ simply to clarify the flow regimes that occur.

$$
\begin{gathered}
t_{m b}=\frac{N_{p}(t)}{q(t)} \\
R N P^{\prime}\left(t_{m b}\right)=\frac{\partial R N P}{\partial \ln \left(t_{m b}\right)}
\end{gathered}
$$

In our constant-BHP linear flow model (Figure 22), we begin with the typical half-slope signature of linear flow. At the onset of fracture interference, the RNP' curve crosses the RNP curve, and then non-monotonically dips back to a half-slope as the long-term late-time linear flow begins. When plotted as $R N P$ and $R N P^{\prime}\left(t_{m b}\right)$ versus $t_{m b}$ (Figure 23), our diagnostic plot looks nearly identical to its constant-rate counterpart (Figure 19). It shows the characteristic linear half-slope, fracture interference unit slope, and long-term half-slope in the same order as we see in the constant-rate model. 


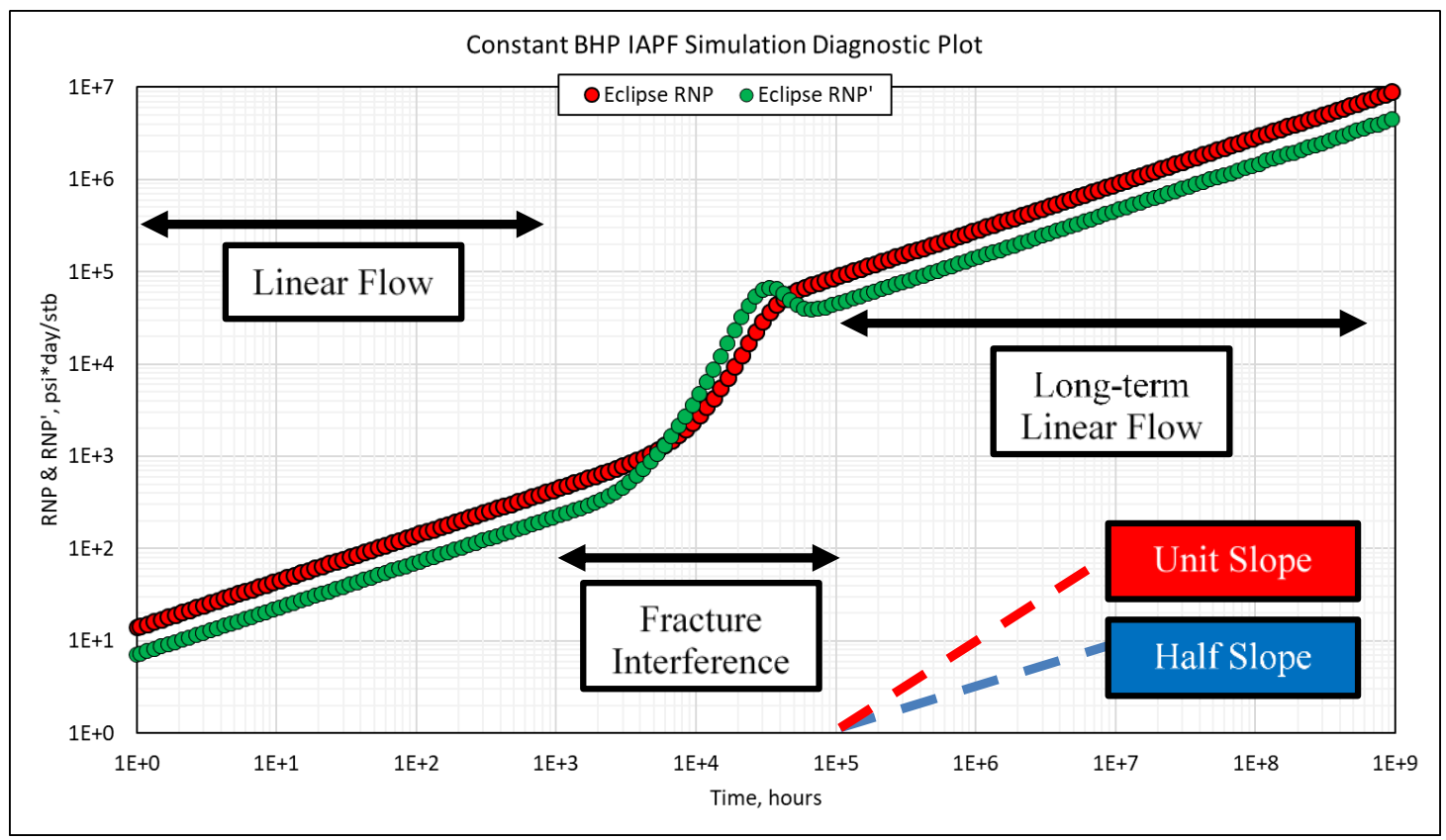

Figure 22 - IALF Constant-BHP Simulation Diagnostic Plot

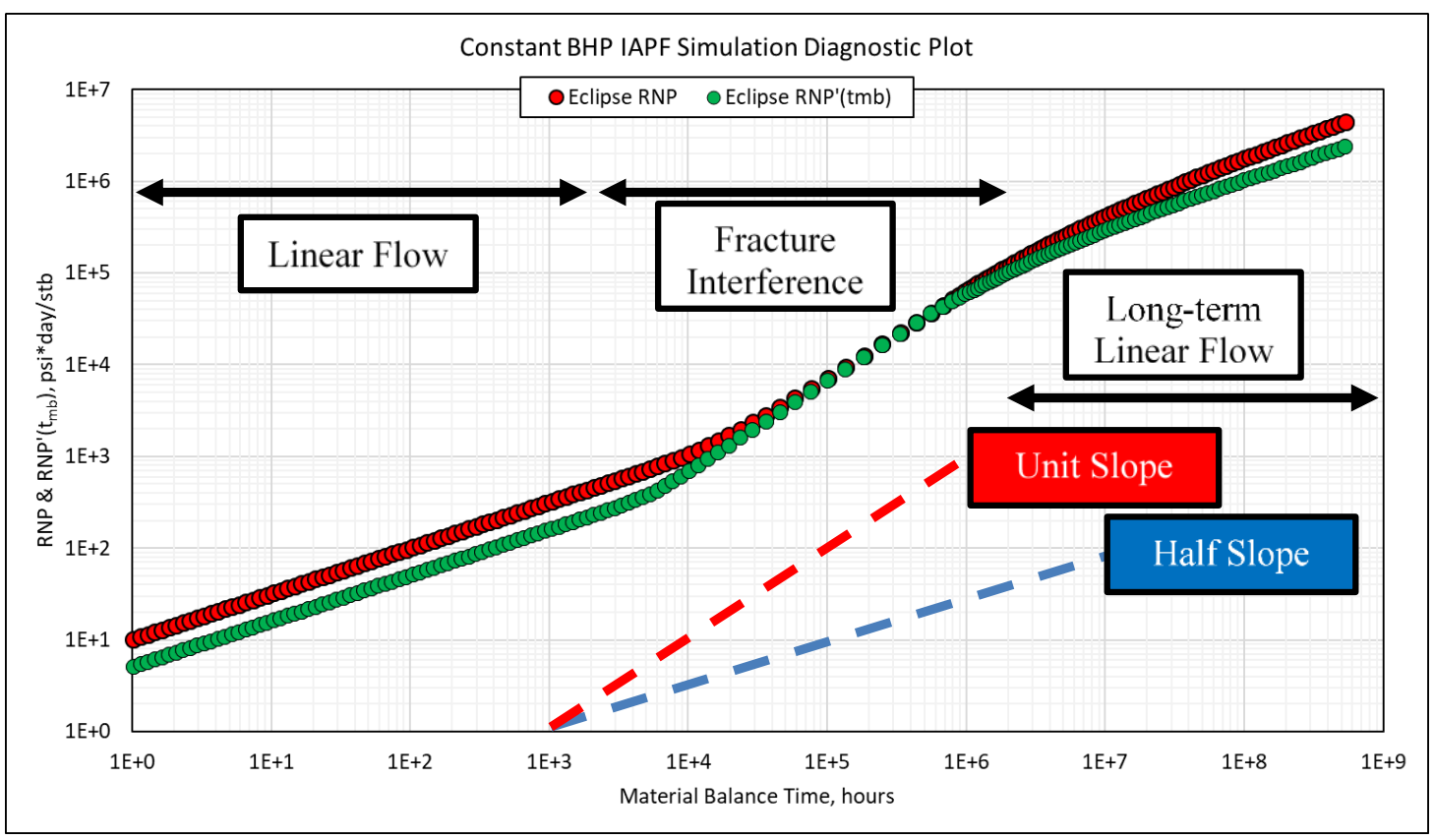

Figure 23 - IALF Constant-BHP Simulation Diagnostic Plot $\left(\mathbf{t}_{\mathrm{mb}}\right)$ 
In the bounded 2-dimensional reservoir case we have the same progression of flow regimes with a constant BHP inner boundary condition as we do for the constantrate case. Once again, the standard diagnostic plot (Figure 24) shows a linear half-slope signature during the early time where linear flow perpendicular to the fractures dominates. We then see the characteristic "dip" signature of a trend towards elliptical flow (Figure 26), which is quickly overtaken by the fracture interference signature. Fracture interference appears as an exponential increase at middle time and ends when the fractures start acting as a single unit and begin transitioning to radial flow. The reservoir boundary signature then comes into play, leading to a pseudo steady state or boundary dominated signature. When plotted as a material balance time plot (Figure 25), we see these same flow regimes but with the more easily identifiable slopes we would expect on the constant-rate plot. 


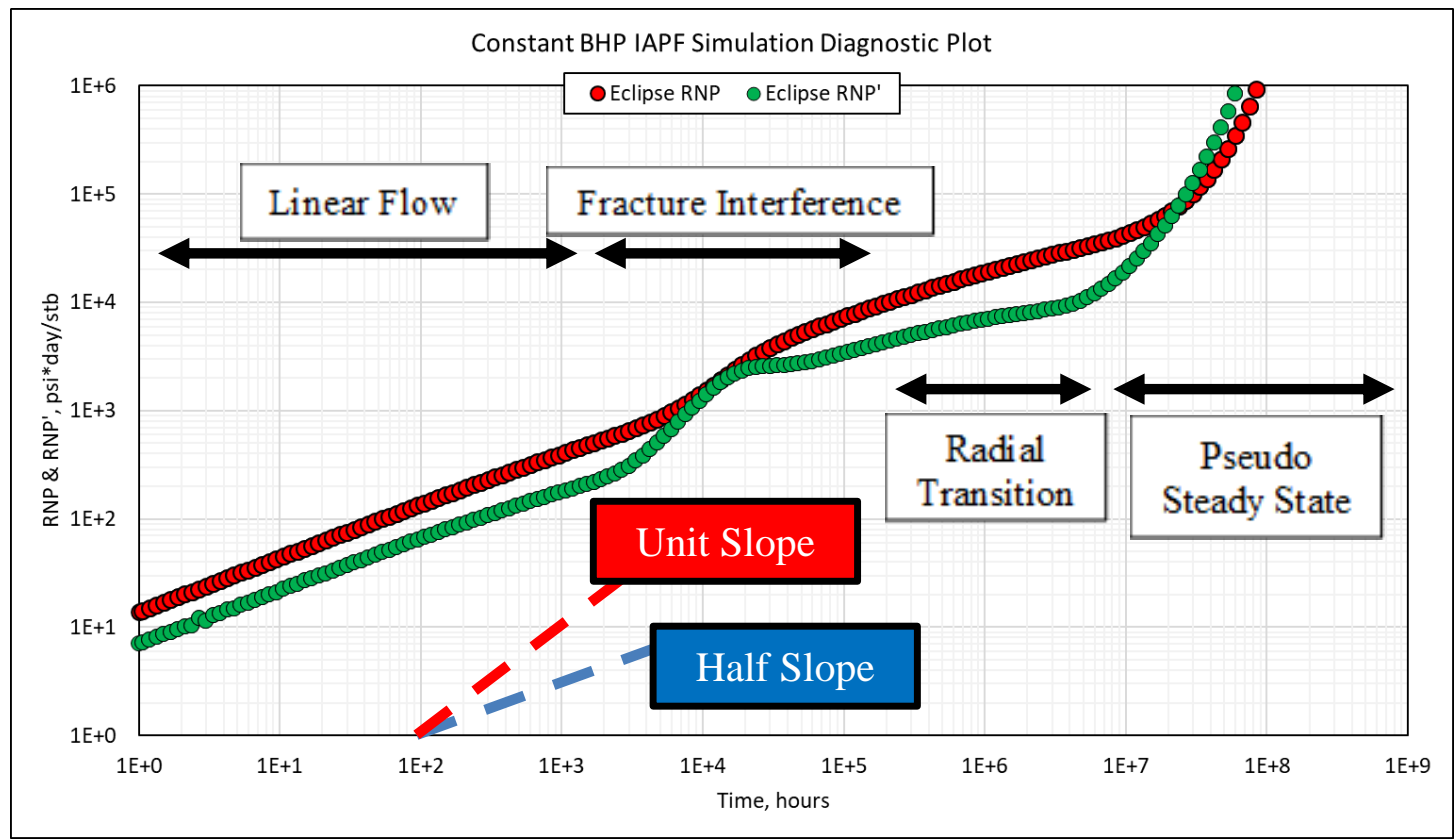

Figure 24 - IAPF Constant BHP Simulation Diagnostic Plot (after Malone et al. (2019)). Copyright 2019, SPE. Reproduced with permission of SPE. Further reproduction prohibited without permission.

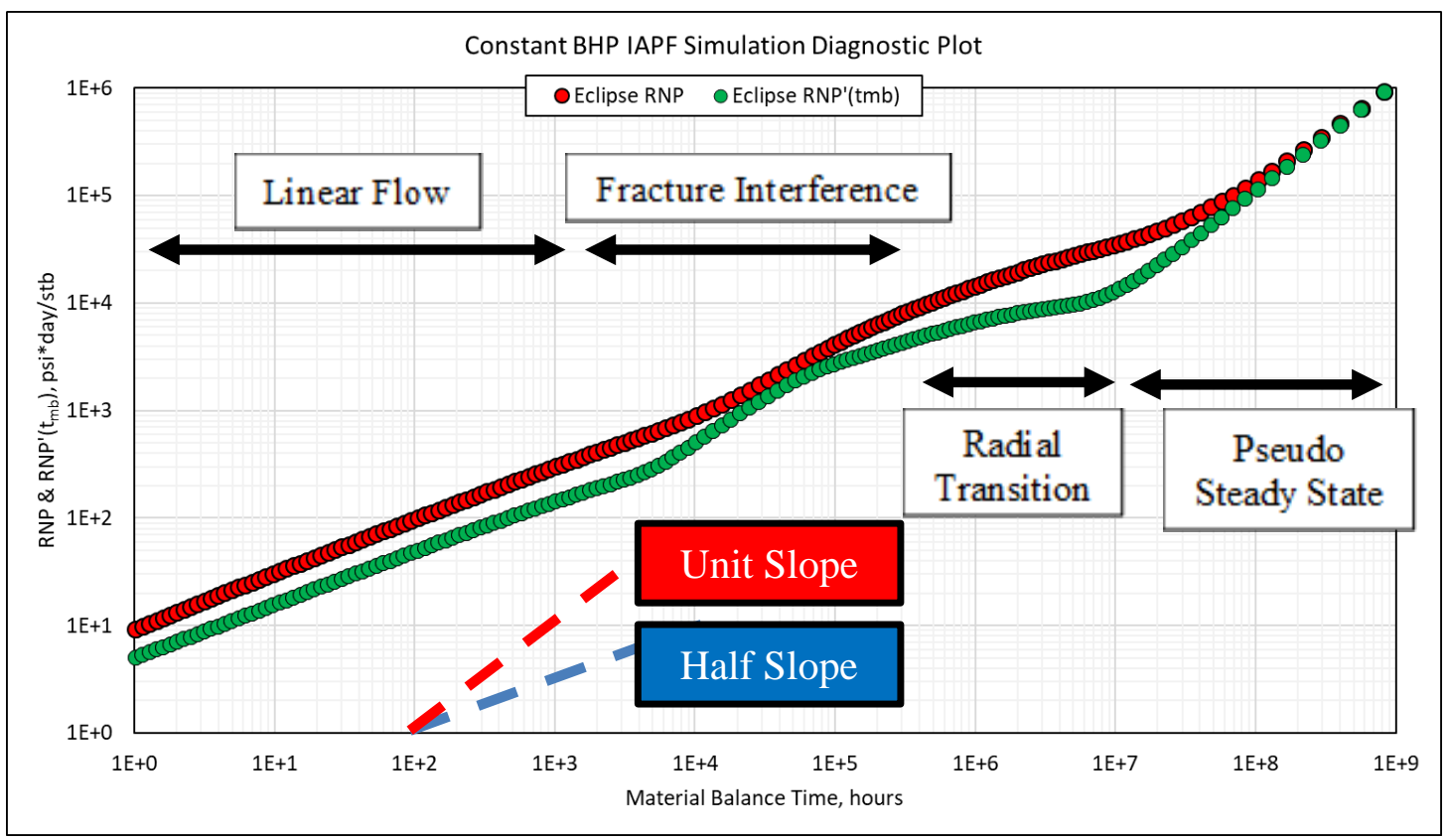

Figure 25 - IAPF Constant BHP Simulation Diagnostic Plot ( $\left.\mathbf{t}_{\mathrm{mb}}\right)$ 


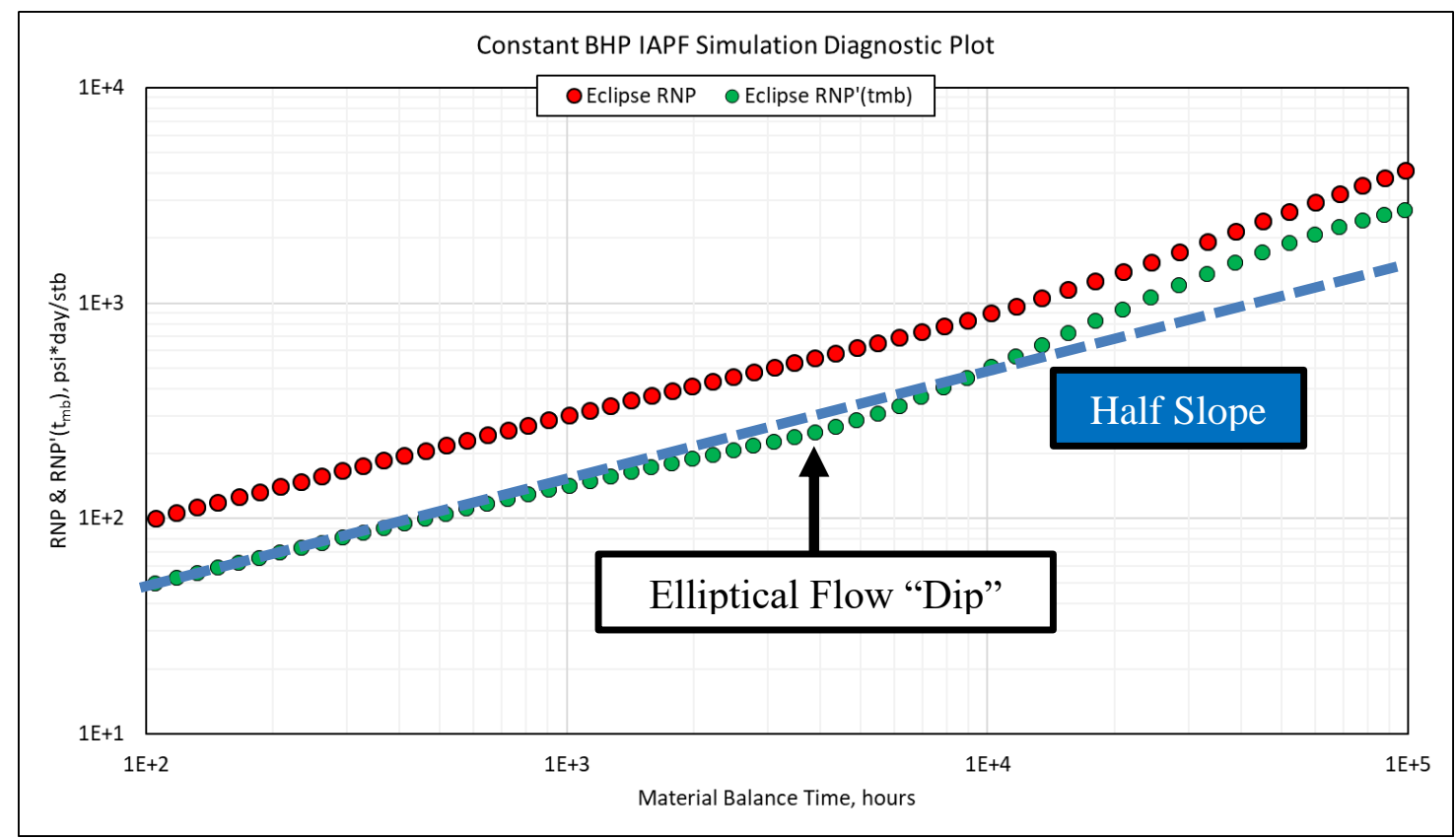

Figure 26 - IAPF Constant BHP Simulation Diagnostic Plot $\left(\mathbf{t}_{\mathrm{mb}}\right)$ Elliptical Flow Dip Signature (zoomed in) (after Malone et al. (2019)). Copyright 2019, SPE. Reproduced with permission of SPE. Further reproduction prohibited without permission.

\section{Results Summary}

The 8 analytic solutions were compared to the commercial simulator reference models, and the qualitative assessment of their approximation quality is shown in Table 2. We prepared analytic solution diagnostic plots using the reservoir properties in Table 1 and show them with their reference model results in this section. 


\begin{tabular}{|ccccc|}
\hline Case & $\begin{array}{c}\text { Inner Boundary } \\
\text { Condition }\end{array}$ & $\begin{array}{c}\text { Unknown Functions } \\
\text { Included }\end{array}$ & Flow Geometry & $\begin{array}{c}\text { Comparison With Reference } \\
\text { Model }\end{array}$ \\
\hline 1 & Variable Rate & A0 & Linear & Identical to reference \\
2 & Constant Rate & A0, A1 & Linear & Identical to reference \\
3 & Variable Rate & A0 & Pillbox & Very close to reference \\
4 & Constant Rate & A0, A1 & Pillbox & Very close to reference \\
5 & Constant BHP & A1 & Linear & Weak approximation \\
6 & Constant BHP & A0, A1 & Linear & Good approximation \\
7 & Constant BHP & A1 & Pillbox & Weak approximation \\
8 & Constant BHP & A0, A1 & Pillbox & Good approximation \\
\hline
\end{tabular}

Table 2 - Analytic Model Results Summary

While looking at the following plots, keep Figure 27, the well diagram for our test model, in mind. "Edge fractures" will refer to the fractures directly adjacent to the toe and heel of the well, fractures F1 and F20. "Central fractures" will refer to the two fractures nearest the very middle of the well's horizontal section, fractures F10 and F11. "Inner fractures" will refer to all the fracture in between the edge fractures, fractures F2 through F19. Note that our analytic solutions do not account for any asymmetrical flow parameters such as friction along the wellbore, so fractures opposite each other relative to the horizontal section's center will exhibit identical flow behavior. More specifically, fractures F1 and F20 will behave the same, F2 and F19 will behave the same, F3 and F18 will be have the same, and so on. On plots involving individual fractures, we plot only 10 curves (i.e. fractures 1 and 20 plot together as one curve), just to make the plots easier to follow. Equivalence within opposing fracture pairs has been confirmed for each case via independent plotting, so we are comfortable plotting individual fracture diagnostics this way. 


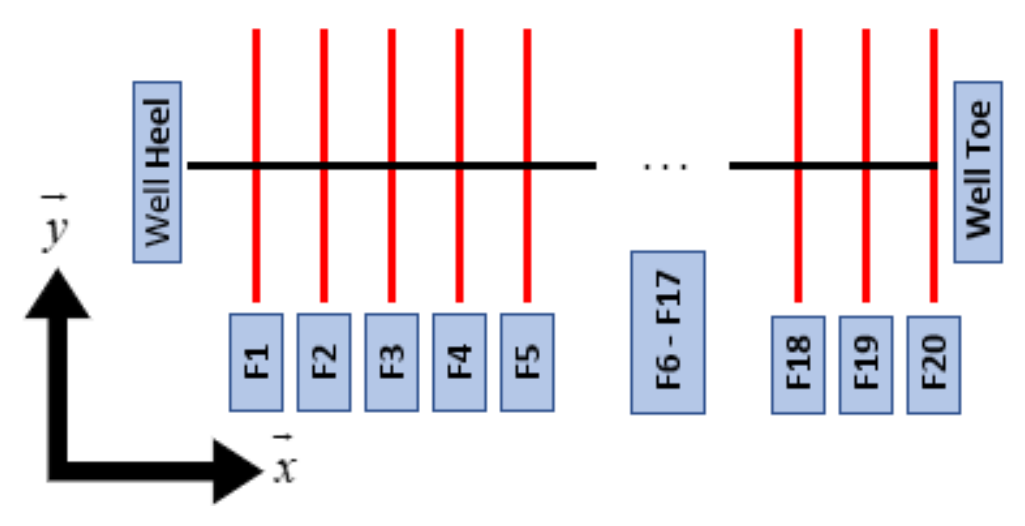

Figure 27- Test Model Diagram - Fracture Numbering

\section{Case 1 - Variable Rate IALF $\left(A_{0}\right)$ - Results}

Case 1's analytic solution's behavior nearly identically mirrored the reference model's behavior (Figure 28). It exhibits early time linear flow shown by a half-slope on our diagnostic plot's RNP' curve. Next, we see a fracture interference period indicated by the nearly-unit slope at middle time. Finally, we see long-term linear flow caused by the 1-dimensional reservoir geometry.

Individual fracture behavior provides interesting insight into the nature of fracture interference and what exactly occurs during SRV drainage. Given the boundary conditions of Case 1, the individual fracture flowrates will vary relative to each other but their sum will remain equal to the total flowrate constraint of the well $(0.1 \mathrm{bbl} / \mathrm{day})$ (Figure 29). At early time when fractures act independently, each fracture contributes an equal portion of the whole well's flow rate. As time progresses to fracture interference, we see the flowrates diverge. The center fractures see a decrease in flowrate as they 
more fully drain their respective drainage volumes. The edge fractures see a growth in flowrate as they access the infinite reservoir volume available along the length of the reservoir.

Individual fracture RNP (Figure 30) and RNP' (Figure 31) behavior also provide information on what individual fractures see as their transients overlap. At early time we see all the fractures independently exhibit linear flow, shown by a half-slope on their RNP' curves. As fracture interference begins and virtual no-flow boundaries form in between fractures, we see all the inner fractures exhibit pseudo-steady state flow, indicated by a unit slope. This continues to infinite time. We see a slight deflection in the RNP' curve of the edge fractures, indicating interaction with the no-flow boundary formed between them and their neighbors (fractures 2 and 18 in this case). A linear halfslope then reforms as the edge fractures drain the infinite linear reservoir.

Interpreting these plots together, we can see what causes the overall well behavior, and how different fractures influence the whole well diagnostic response. At early time, fractures act independently, and all exhibit linear flow, which is reflected in the early time whole-well behavior. As fracture interference begins and the inner fractures exhibit pseudo-steady state flow, the whole-well response reflects that trend. As time progresses, the edge fractures continue to see linear flow, and the inner fractures continue to see pseudo-steady state flow, but their proportional changes in rate adjust their respective influences on the whole-well behavior. Immediately as fracture interference begins, individual fractures rates remain similar, so the whole-well response follows the majority fracture response - pseudo-steady state flow. However, the edge 
fracture rates begin to climb, and inner fracture rates decay, so we see the edge fracture behavior dominate the whole-well behavior as we progress into very late time.

Asymptotic solution parameter $A_{0}$ is also shown on a by-fracture basis (Figure 32). This plot primarily helps in evaluating the stability of our solution, and in multiterm approximations it allows us to see which asymptotic terms dominate the solution. In this case we have only one term $\left(A_{0}\right)$, and it shows a monotonic decrease through time. A divergence occurs between the edge and inner fractures as fracture interference begins, and we see all the non-central inner fracture $A_{0}$ terms eventually converge upon the same value as the central fracture $A_{0}$ terms.

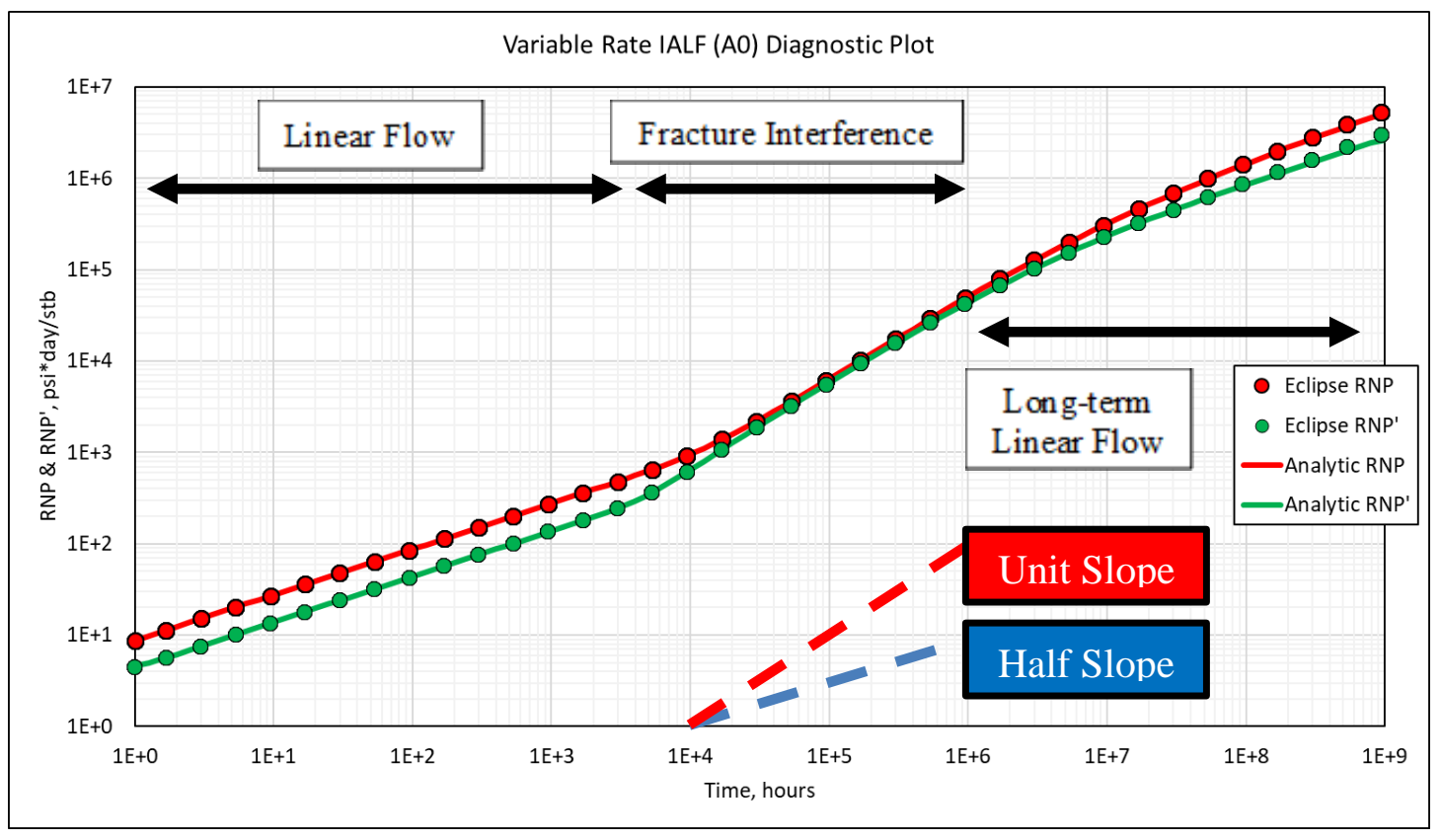

Figure 28 - Case 1 Diagnostic Plot Comparison with Reference Model 


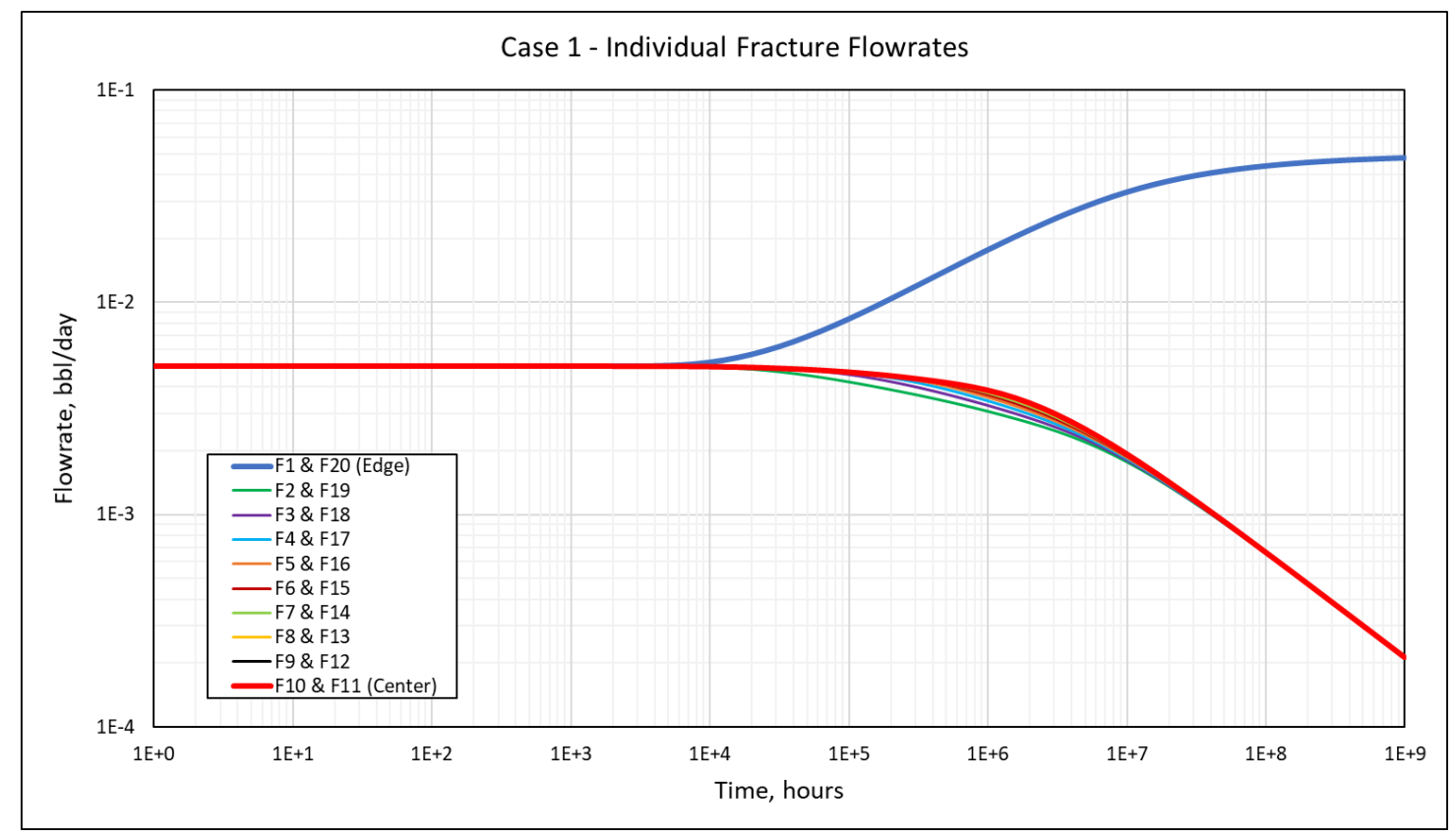

Figure 29 - Case 1 - Individual Fracture Flowrates

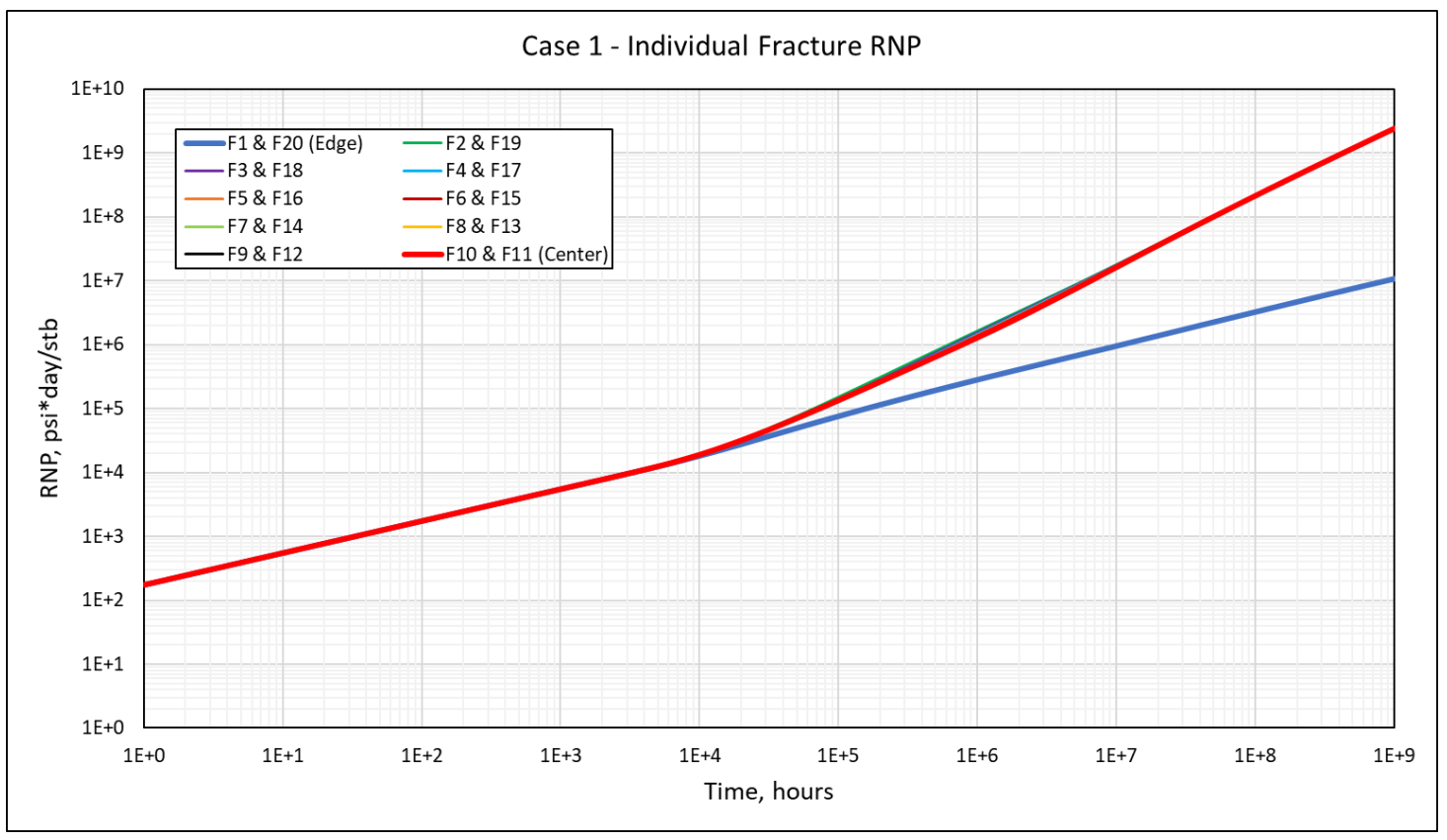

Figure 30 - Case 1 - Individual Fracture RNP 


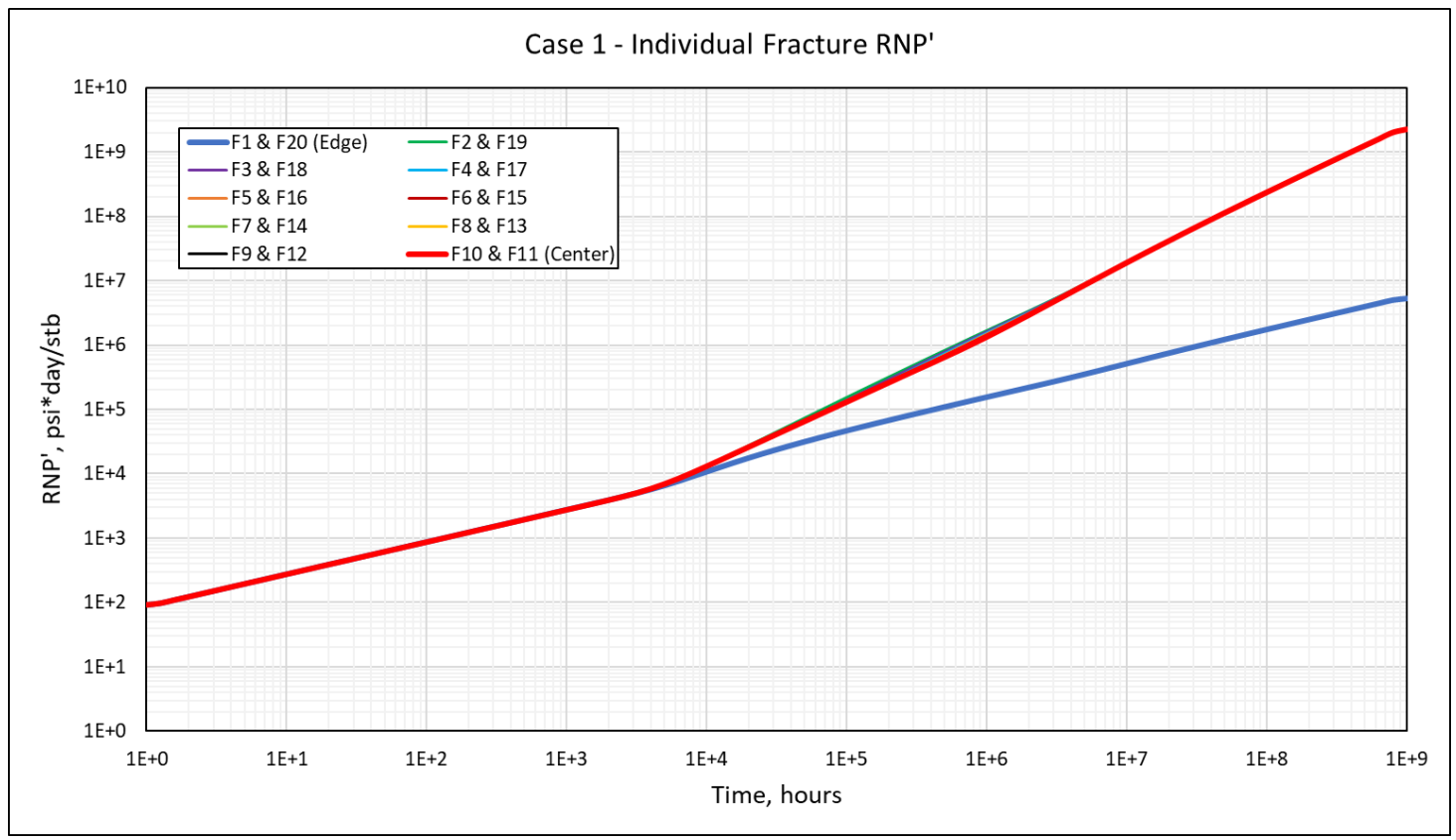

Figure 31 - Case 1 - Individual Fracture RNP'

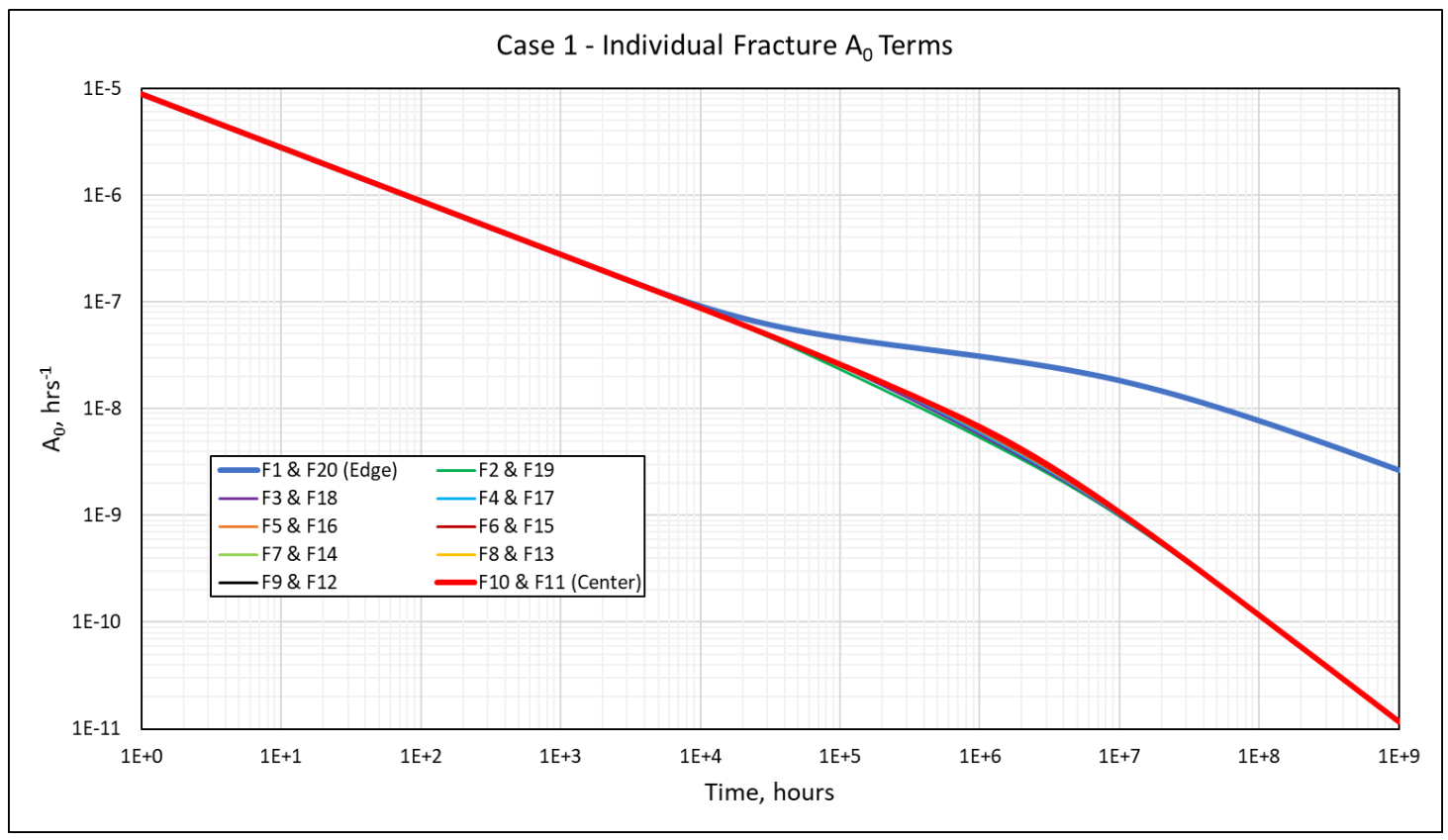

Figure 32 - Case 1 - Individual Fracture Ao Terms 
Case 2 - Constant Rate IALF $\left(A_{0}\right.$ and $\left.A_{1}\right)$ - Results

Case 2's analytic solution's behavior also nearly identically reflects the reference model's behavior (Figure 33). As with the reference model, it progresses from early linear flow to fracture interference, or approximate pseudo-stead state flow, then back to late time linear flow.

Given that Case 2 is identical to Case 1 except in that it applies an additional asymptotic solution unknown function $\left(A_{1}\right)$, its individual fracture diagnostics are nearly identical to Case 1's with one exception. Case 2's wellbore rate derivative constraint and additional unknown function drive all fractures to one of two solutions. The edge fractures (F1 and F20) see one response, and all the other fractures (F2-F19) see another. This is true of the rate response (Figure 34), the RNP response (Figure 35), and the RNP' response (Figure 36).

We may interpret Case 2's diagnostics similarly to how we interpret Case 1's. At early time the fractures flow independently exhibiting linear flow which is reflected in the whole well response. Once fracture interference begins, the inner fractures encounter pseudo-steady state flow, and this response dominates the whole-well response until the inner fracture rates decay, and we see the edge fracture linear signature dominate into very late time.

In this case the solution is primarily driven by the first unknown function, $A_{0}$, which shows a constant decay for all fractures until interference begins, and then a separation in responses afterwards (Figure 37). The second unknown function, $A_{1}$, 
comes into play only during fracture interference, and has a varying magnitude for each fracture (Figure 38). Its apparent effect is to decrease the flux to the edge fractures (shown by its negative value), allowing the inner fractures to take some additional flux during the interference period (which is why they are all equal through all time).

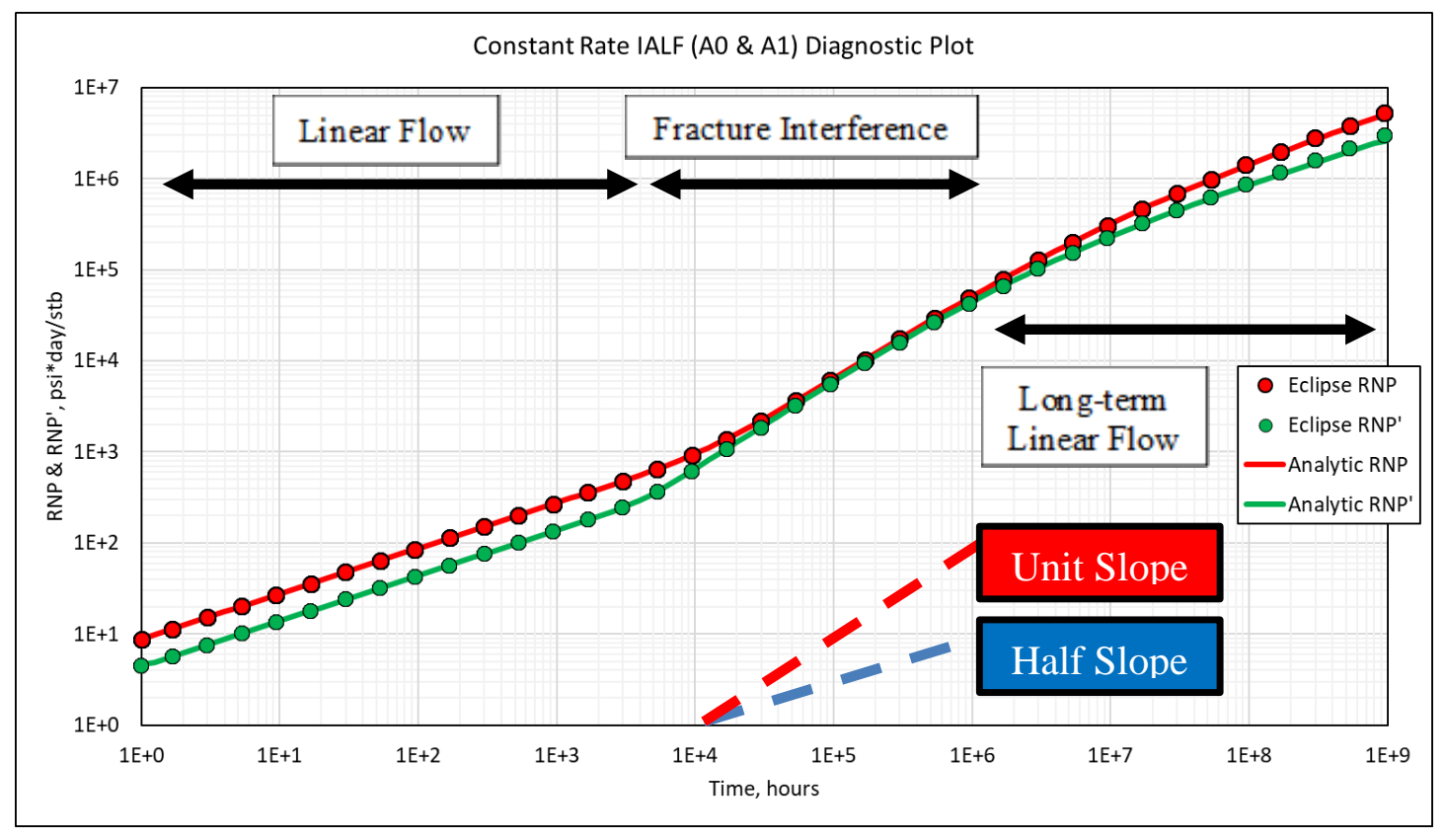

Figure 33 - Case 2 Diagnostic Plot Comparison with Reference Model 


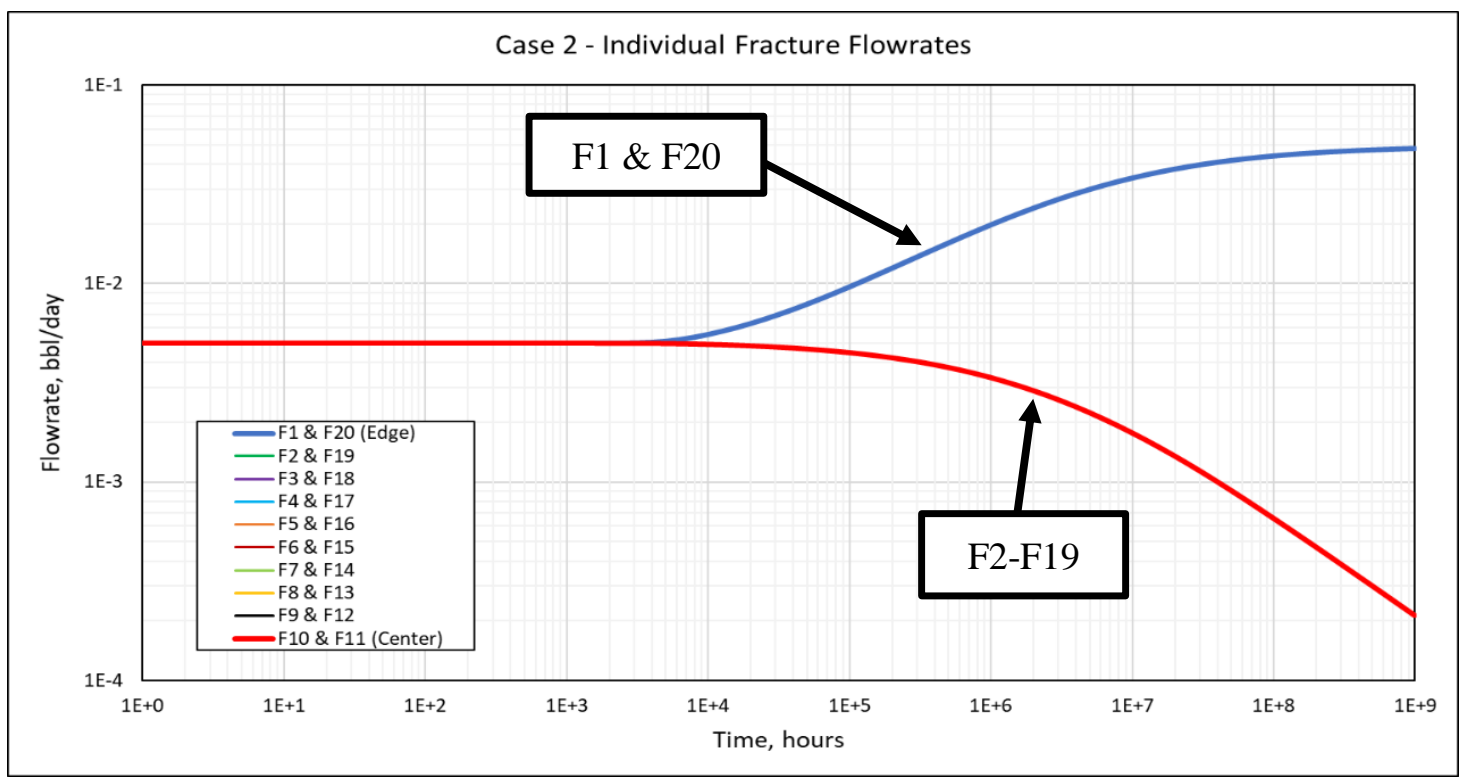

Figure 34 - Case 2 - Individual Fractures Flowrates

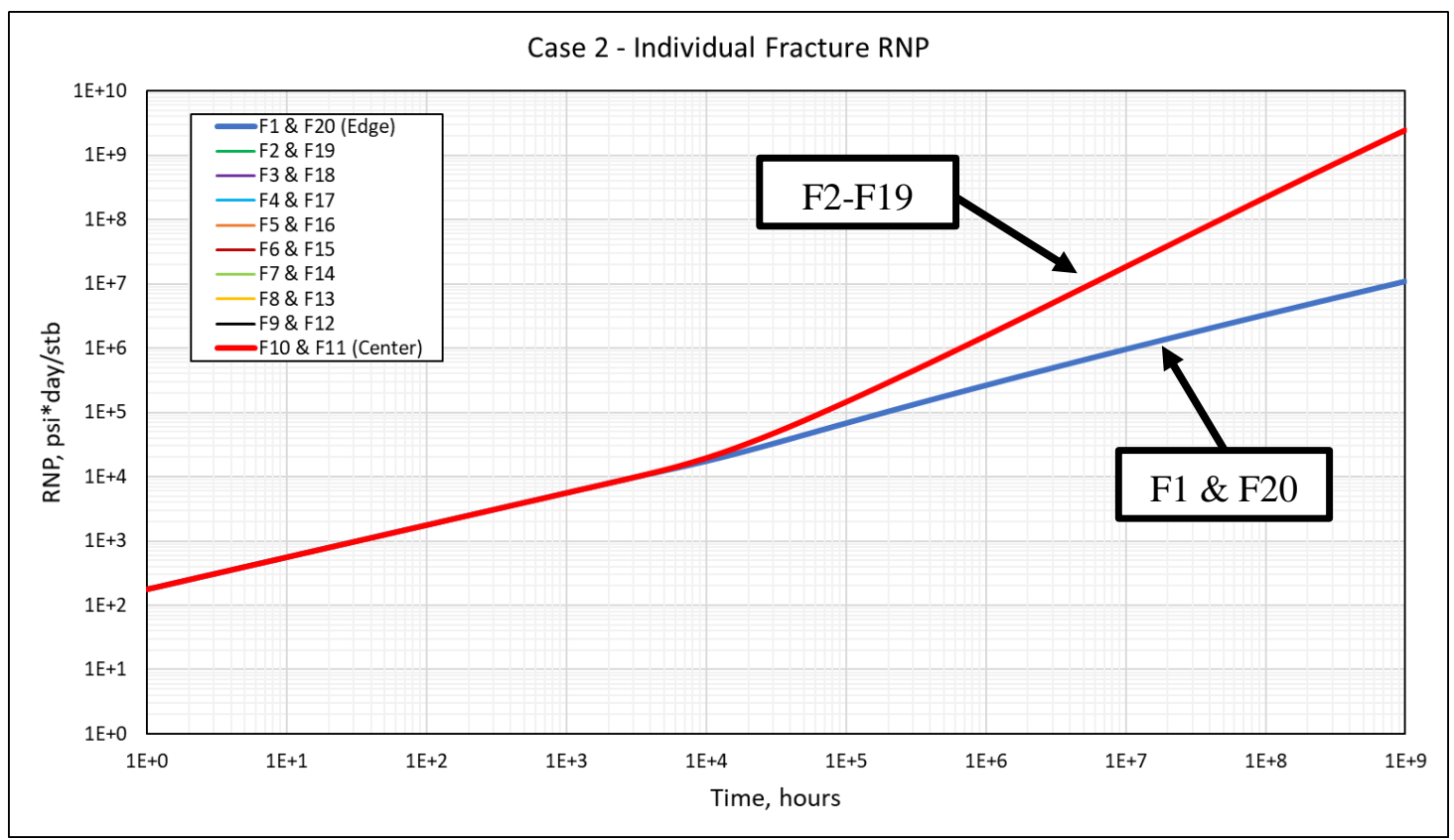

Figure 35 - Case 2 - Individual Fracture RNP 


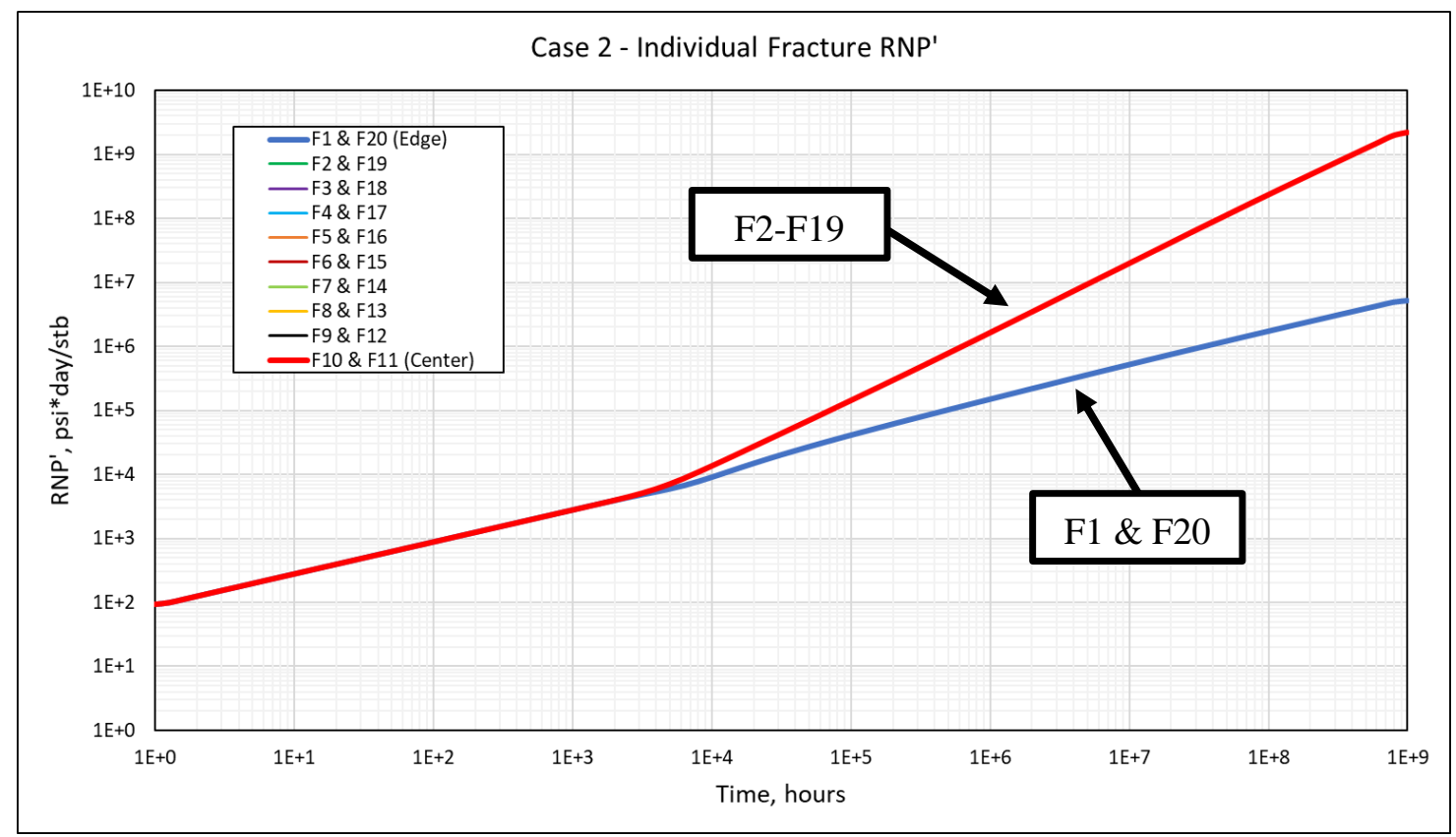

Figure 36 - Case 2 - Individual Fracture RNP'

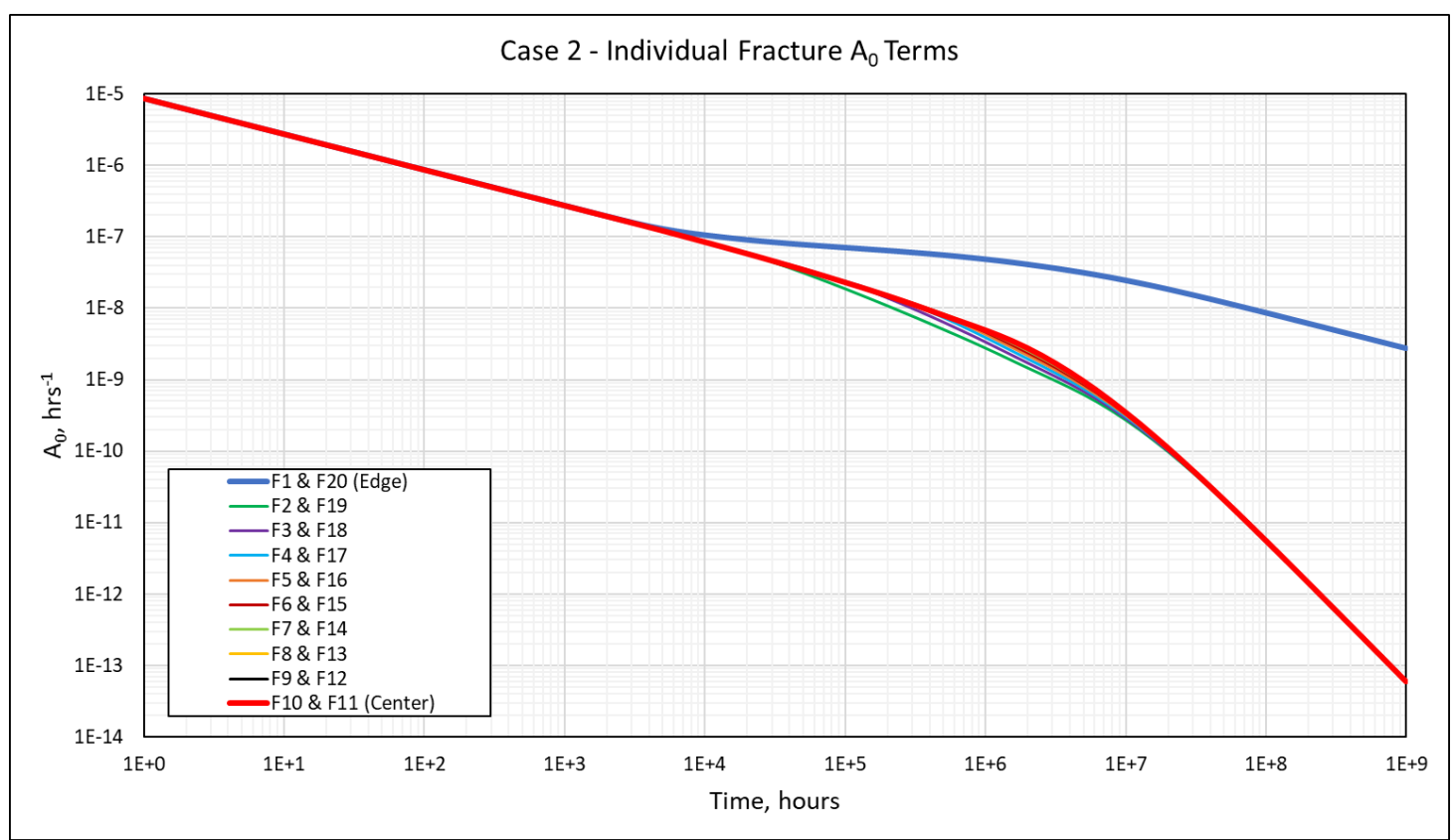

Figure 37 - Case 2 - Individual Fracture Ao Terms 


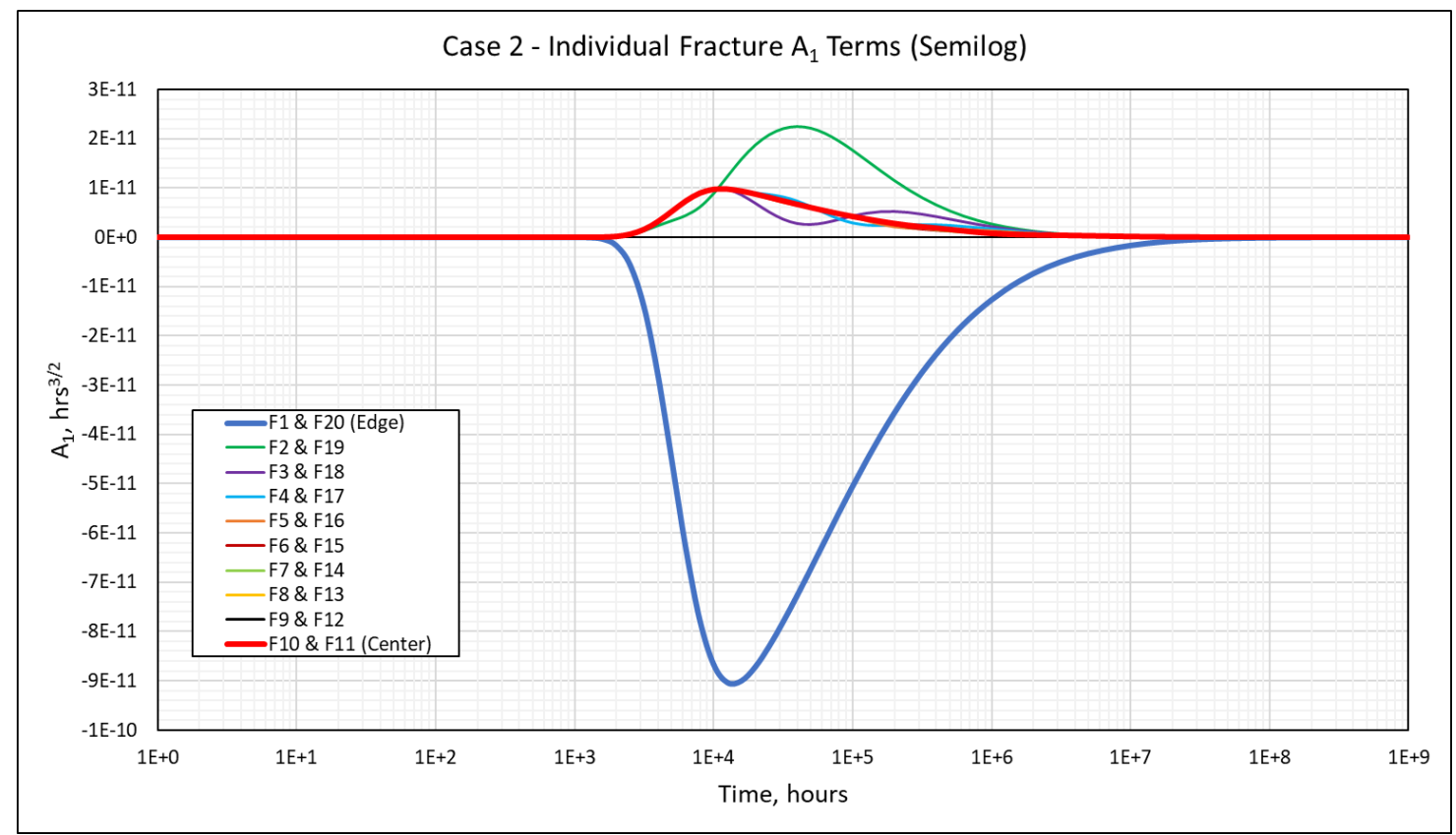

Figure 38 - Case 2 - Individual Fracture A1 Terms

Case 3 - Variable Rate IAPF (Ao) - Results

The analytic construction for Case 3 managed to closely approximate the results from our 2-dimensional reference model. Early time linear flow, fracture interference, and the eventual transition to infinite-acting compound radial flow are all exhibited by the analytic model (Figure 39). After the onset of fracture interference, the solutions show some offset primarily because the "pillbox" geometry assumption does not exactly match the true progression of flow regimes for a single fracture. In reality, we see a brief period of pillbox flow followed by elliptical and then radial flow (Lee et al. 2003). In our approximation, there is no true elliptical flow, leading to the discrepancy we see between the reference model and our analytic solution after linear flow ends. Another late time 
divergence at approximately $10^{7}$ hours occurs because our reference model includes a reservoir boundary, but our analytic model assumes an infinite reservoir.

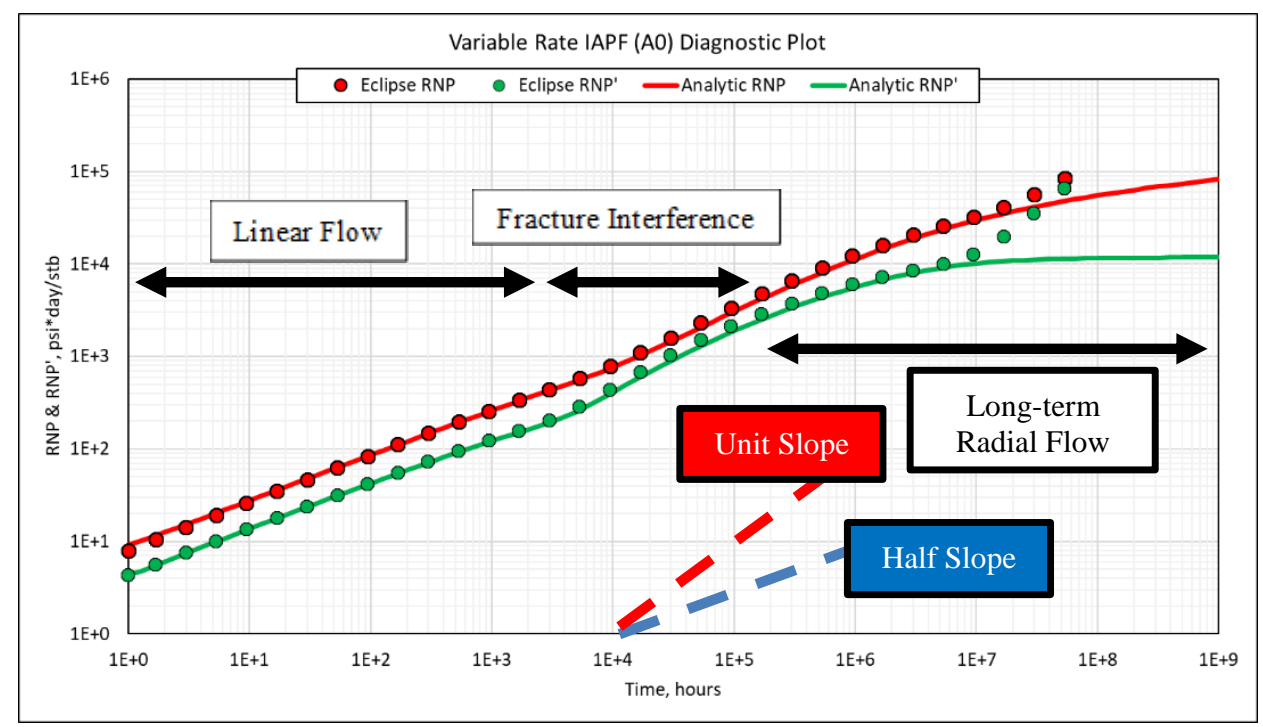

Figure 39 - Case 3 Diagnostic Plot Comparison with Reference Model (after Malone et al. (2019)). Copyright 2019, SPE. Reproduced with permission of SPE. Further reproduction prohibited without permission.

The reader is reminded that the 2-dimensional reference model shows a preinterference "dip" signature, where elliptical flow is shortly seen before the period of fracture interference begins. This pre-interference "dip" signature (Figure 21) is replicated by the analytic solution. It represents the growth of individual fracture drainage volumes to the point that flow to the fracture tips should no longer be considered negligible relative to flow perpendicular to the fracture face. In a case with 
more widely-spaced fractures, this dip will become more apparent, and could even evolve into full radial flow with a high enough fracture spacing.

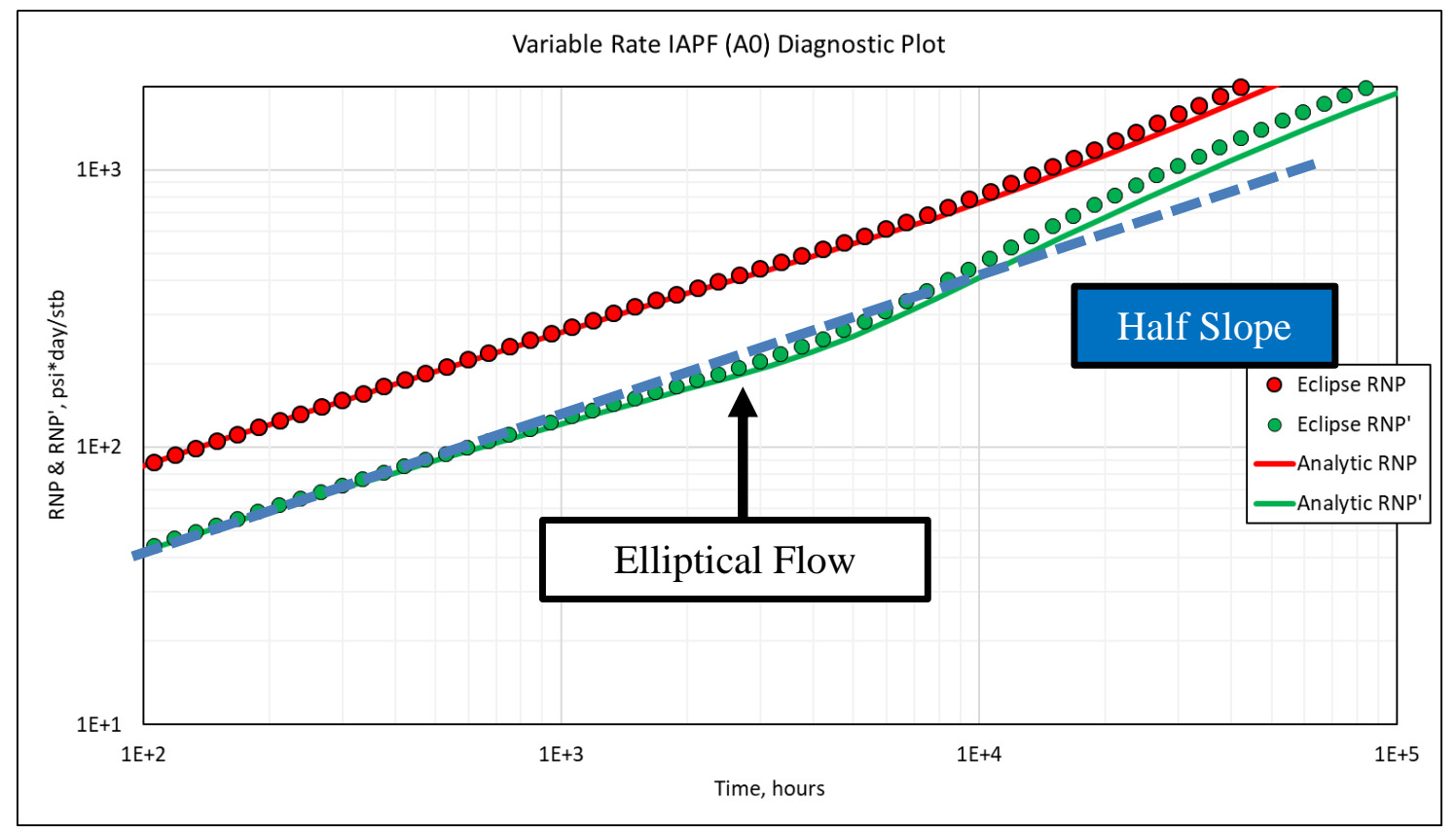

Figure 40 - Case 3 Diagnostic Plot - Elliptical "Dip" Signature (after Malone et al. (2019)). Copyright 2019, SPE. Reproduced with permission of SPE. Further reproduction prohibited without permission.

With our analytic model, we may also observe interesting results on an individual fracture basis. Again, fracture flowrates act independently of each other at early time, all contributing an equal proportion of the total wellbore flow constraint (Figure 41). Once fracture interference begins, flowrates diverge. With the greatest access to the infinite reservoir, the edge fractures' flow contribution increases until reaching an upper limit, and evens out once late-time radial flow sets in. Inner fractures appear to drop in 
flowrate after interference begins, and then flatline in the same way. As we move further towards the center of the horizontal section, this flatline flowrate value decreases, and the center fractures see the least amount of flow.

Taking a look at our RNP and RNP' plots (Figure 42 and Figure 43, respectively), we can make out the progression of flow regimes for each fracture. The edge fractures see the early period of linear flow, the pre-interference "dip", and a boundary deflection caused by the virtual no-flow boundary imposed between the edge fractures and their neighbors. As we progress to late time, the edge fractures see a zeroslope, indicating that their flow has evolved to full radial flow. The inner fractures also see the early-time linear flow period, and the pre-interference "dip". Once interference sets in, they exhibit the approximate pseudo-steady state unit slope. Each inner fracture then eventually progresses to long-term radial flow. The closer a fracture is to the edge fractures, the earlier it begins to see radial flow.

In Case 3 we use a single-term approximation ( $A_{0}$ only). $A_{0}$ shows a somewhat muted response in this case. It monotonically decreases, equally for each fracture, until fracture interference begins. At that point, the same monotonic decrease continues, but the slope decreases for inner fractures more quickly than for the edge fractures. 


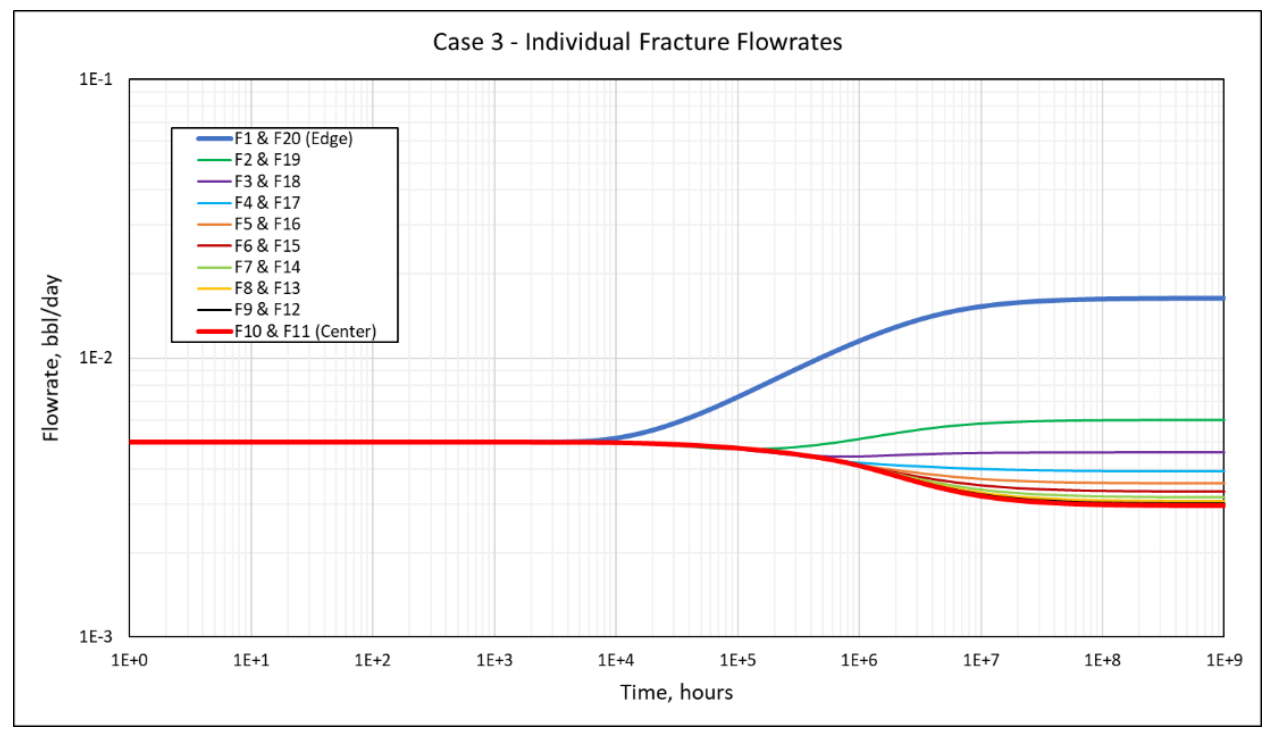

Figure 41 - Case 3 - Individual Fractures Flowrate (after Malone et al. (2019)). Copyright 2019, SPE. Reproduced with permission of SPE. Further reproduction prohibited without permission.

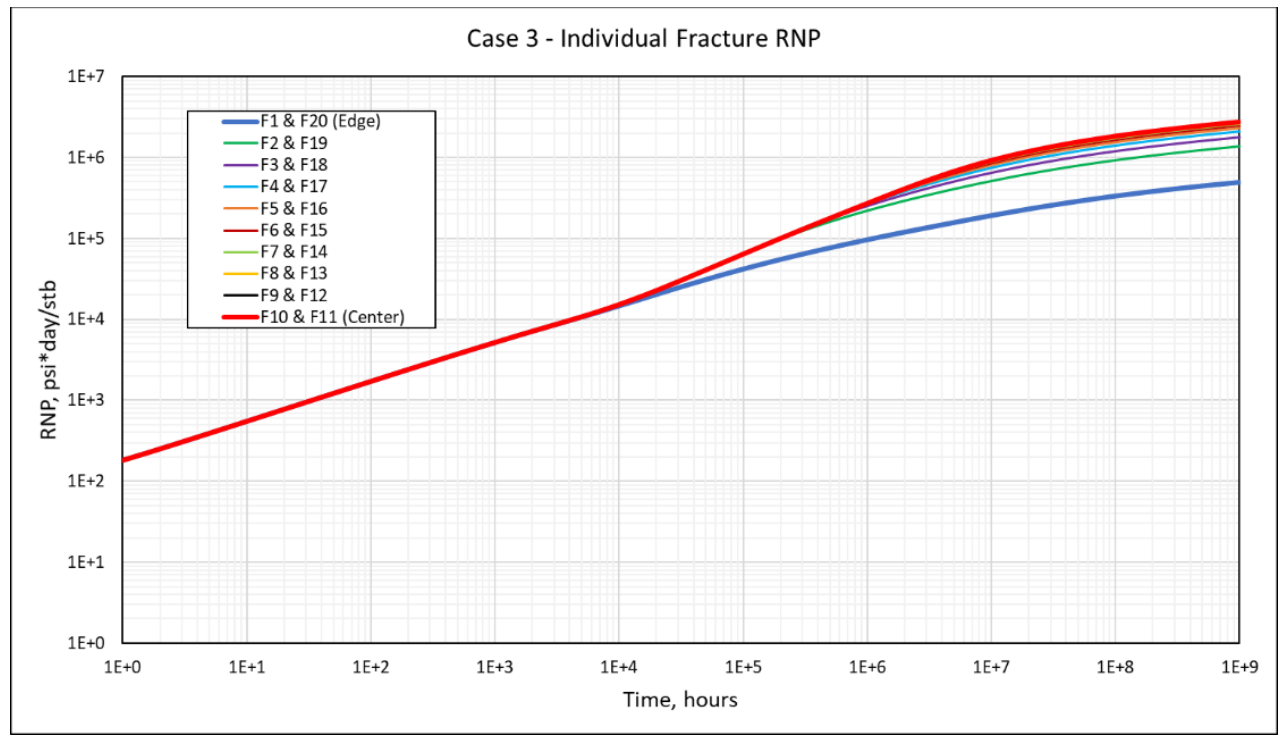

Figure 42 - Case 3 - Individual Fracture RNP (after Malone et al. (2019)). Copyright 2019, SPE. Reproduced with permission of SPE. Further reproduction prohibited without permission. 


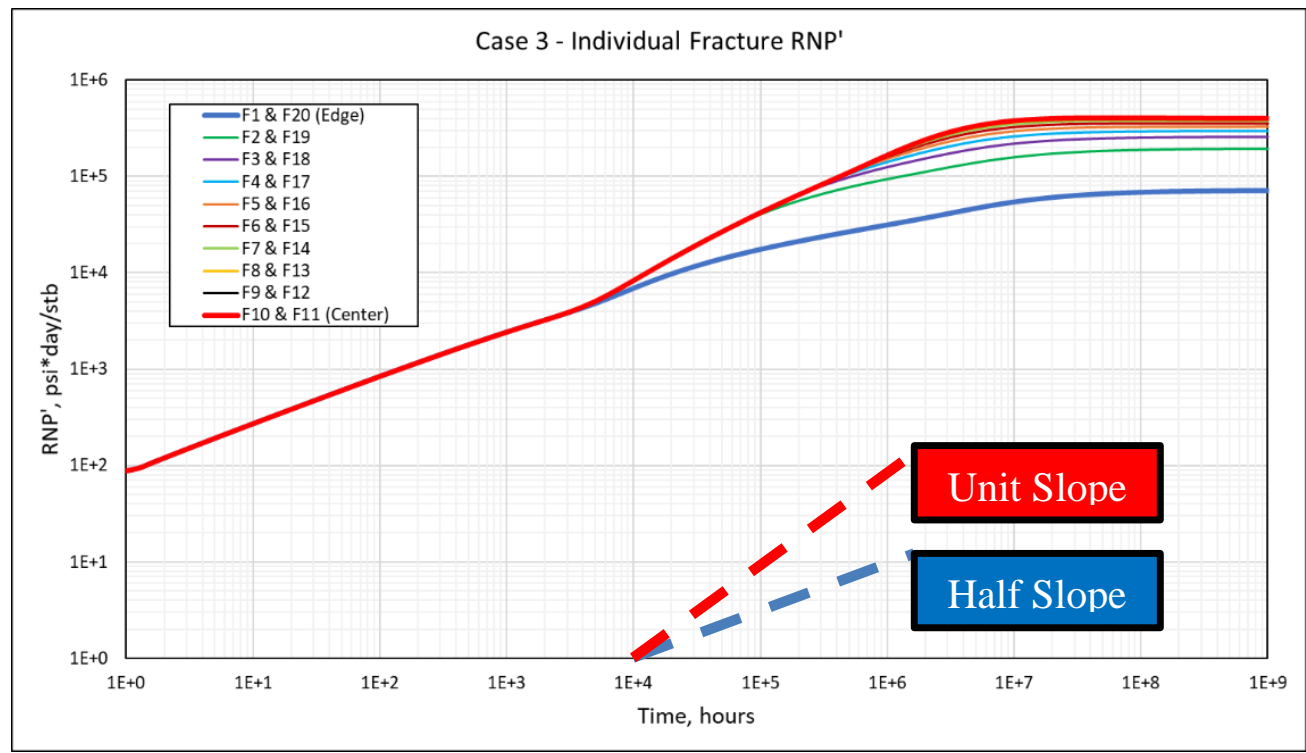

Figure 43 - Case 3 - Individual Fracture RNP' (after Malone et al. (2019)). Copyright 2019, SPE. Reproduced with permission of SPE. Further reproduction prohibited without permission.

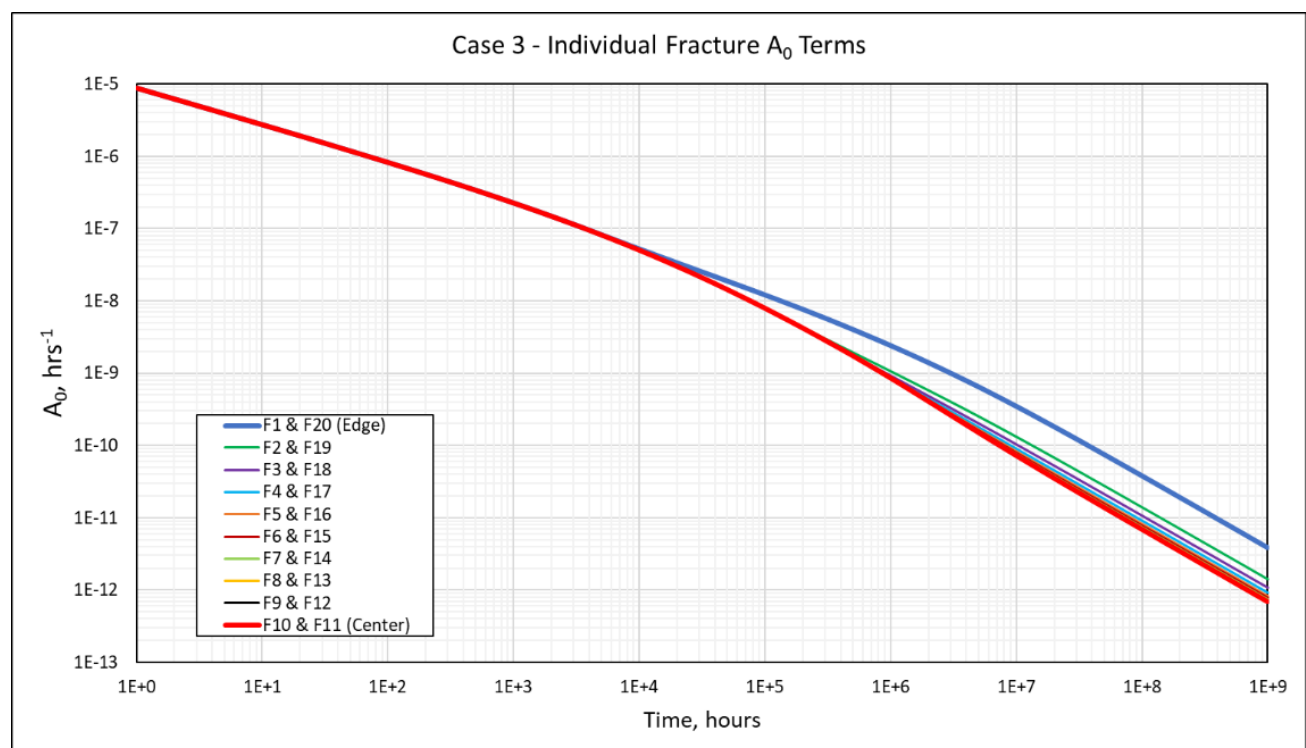

Figure 44 - Case 3 - Individual Fracture Ao Terms (after Malone et al. (2019)). Copyright 2019, SPE. Reproduced with permission of SPE. Further reproduction prohibited without permission. 


\section{Case 4 - Constant Rate IAPF $\left(A_{0}\right.$ and $\left.A_{l}\right)$ - Results}

Case 4's results differ little from Case 3's results. The main difference between Cases 3 and 4 comes in the solution - Case 4 includes the additional asymptotic unknown function, $A_{1}$, and the additional constraint imposed on the derivative of the wellbore flux. Case 4's analytic solution shows good agreement with the reference model results, and encounters the same discrepancies seen between the reference model and Case 3. Initial linear flow seems to match the reference model identically. After fracture interference our analytic solution carries the same overall character but has a consistent slight negative offset from our reference model (Figure 45) and does not model the late-time pseudo-steady state behavior because of its infinite reservoir assumption. Case 4's analytic solution also manages to capture the pre-interference “dip" signature seen in the reference model (Figure 46).

Similarly, to what we see in Case 3, individual fractures contribute equal proportions of the total wellbore flux up until the time when fractures begin to interact (Figure 47). After that we see the edge fracture rates increase up to a maximum, and then the inner fracture flowrates decrease and flatline at some value that decreases as the fracture is closer to the center of the horizontal section. The character of the RNP curves shows very little character (Figure 48), but the RNP' curves show a similar progression of flow regimes to what we saw in Case 3 (Figure 49). The fractures flow independently, exhibiting the linear flow half-slope up until the pre-interference "dip" and subsequent fracture interference. For this case, the dip appears more dramatic for the edge fractures. 
After fracture interference ends, each fracture eventually stabilizes to a period of longterm radial flow.

Solutions are dominated by the $A_{0}$ terms which monotonically decrease through all time and show some "fanning" out during the period of fracture interference (Figure 50). The $A_{1}$ terms appear to influence the solution only during the fracture interference period, and will decrease the flux to the edge fractures while increasing the flux to inner fractures (Figure 51).

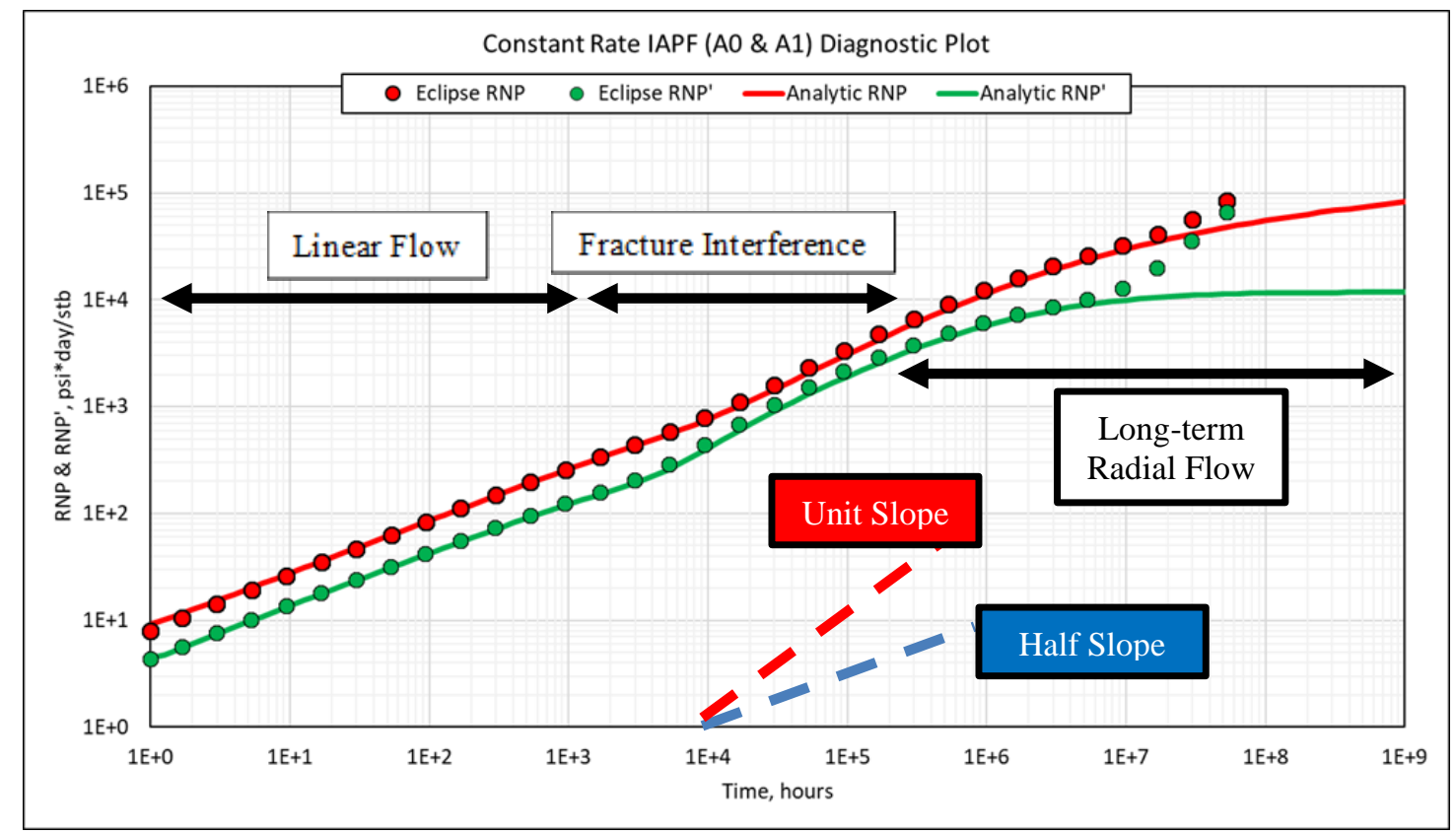

Figure 45 - Case 4 Diagnostic Plot Comparison with Reference Model (after Malone et al. (2019)). Copyright 2019, SPE. Reproduced with permission of SPE. Further reproduction prohibited without permission. 


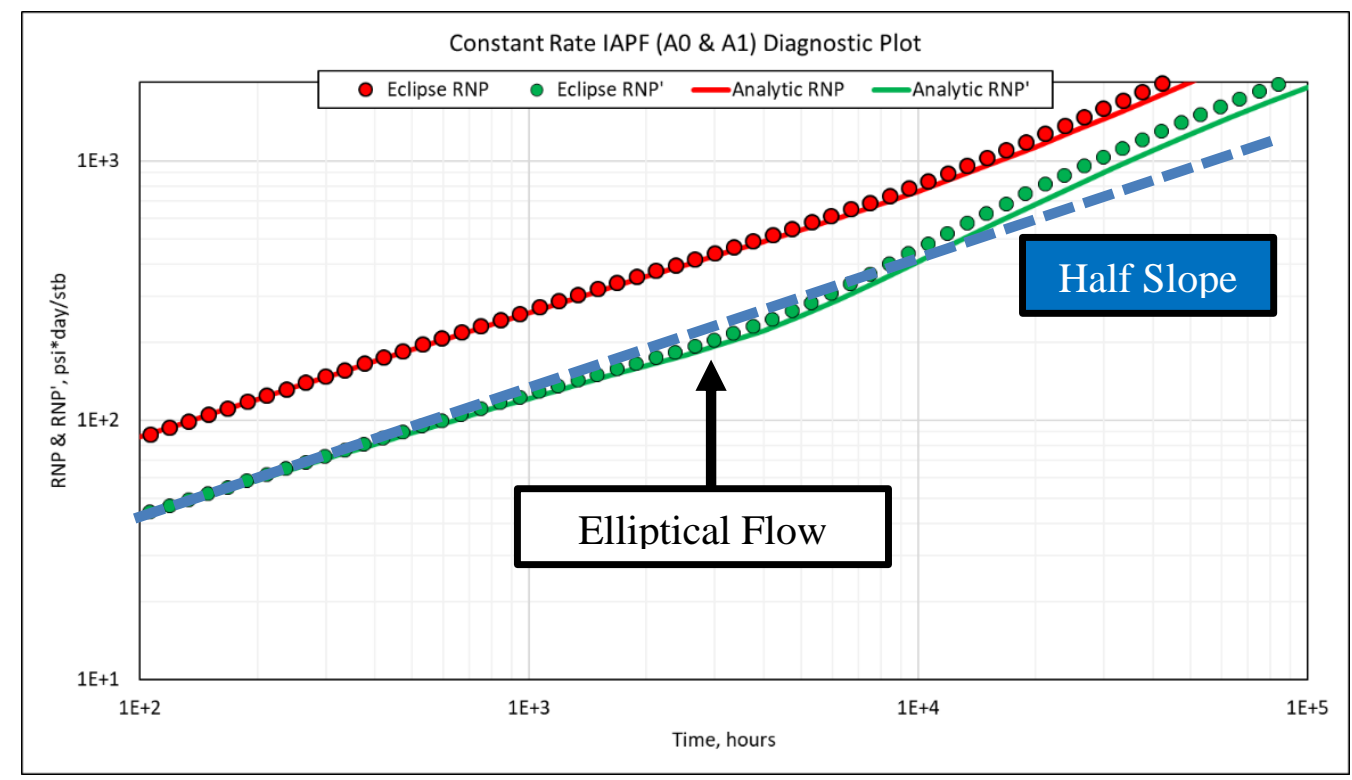

Figure 46 - Case 4 - Elliptical Flow Dip Signature (after Malone et al. (2019)). Copyright 2019, SPE. Reproduced with permission of SPE. Further reproduction prohibited without permission.

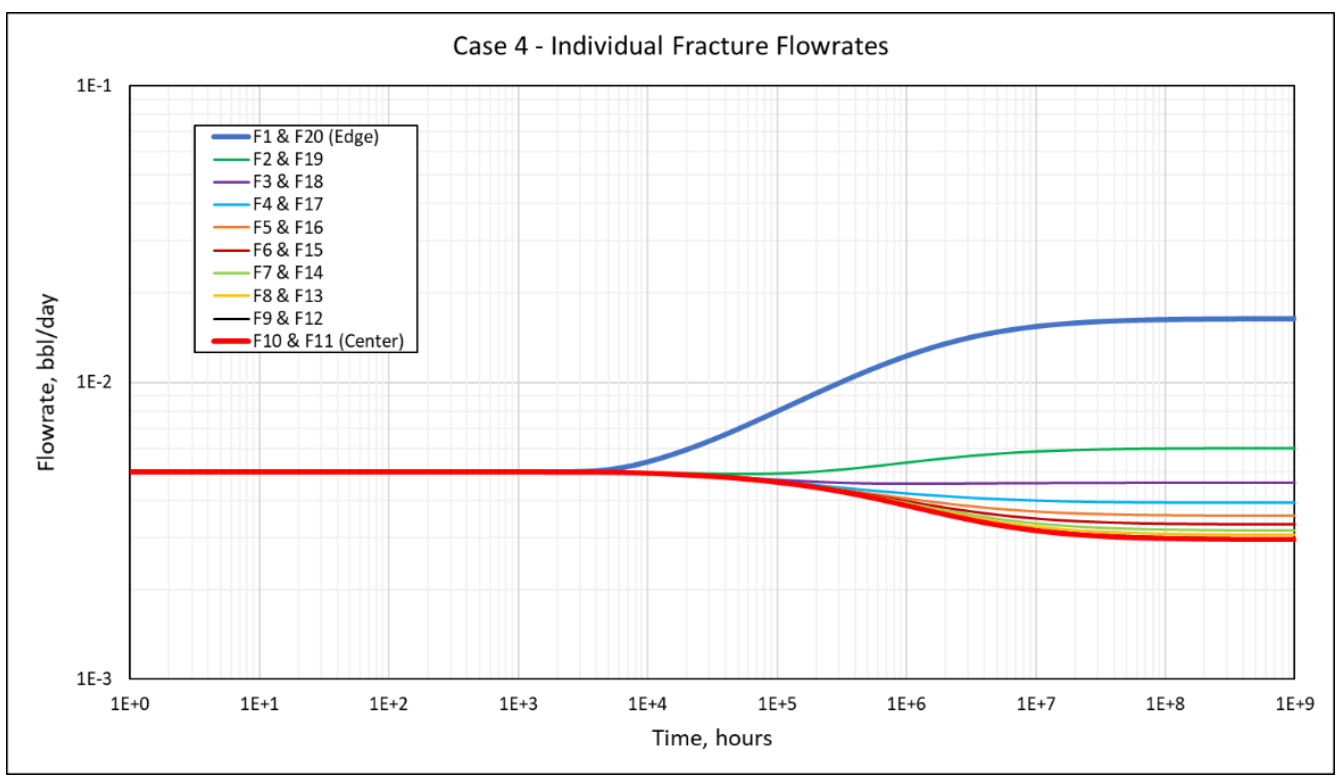

Figure 47 - Case 4 - Individual Fractures Flowrates 


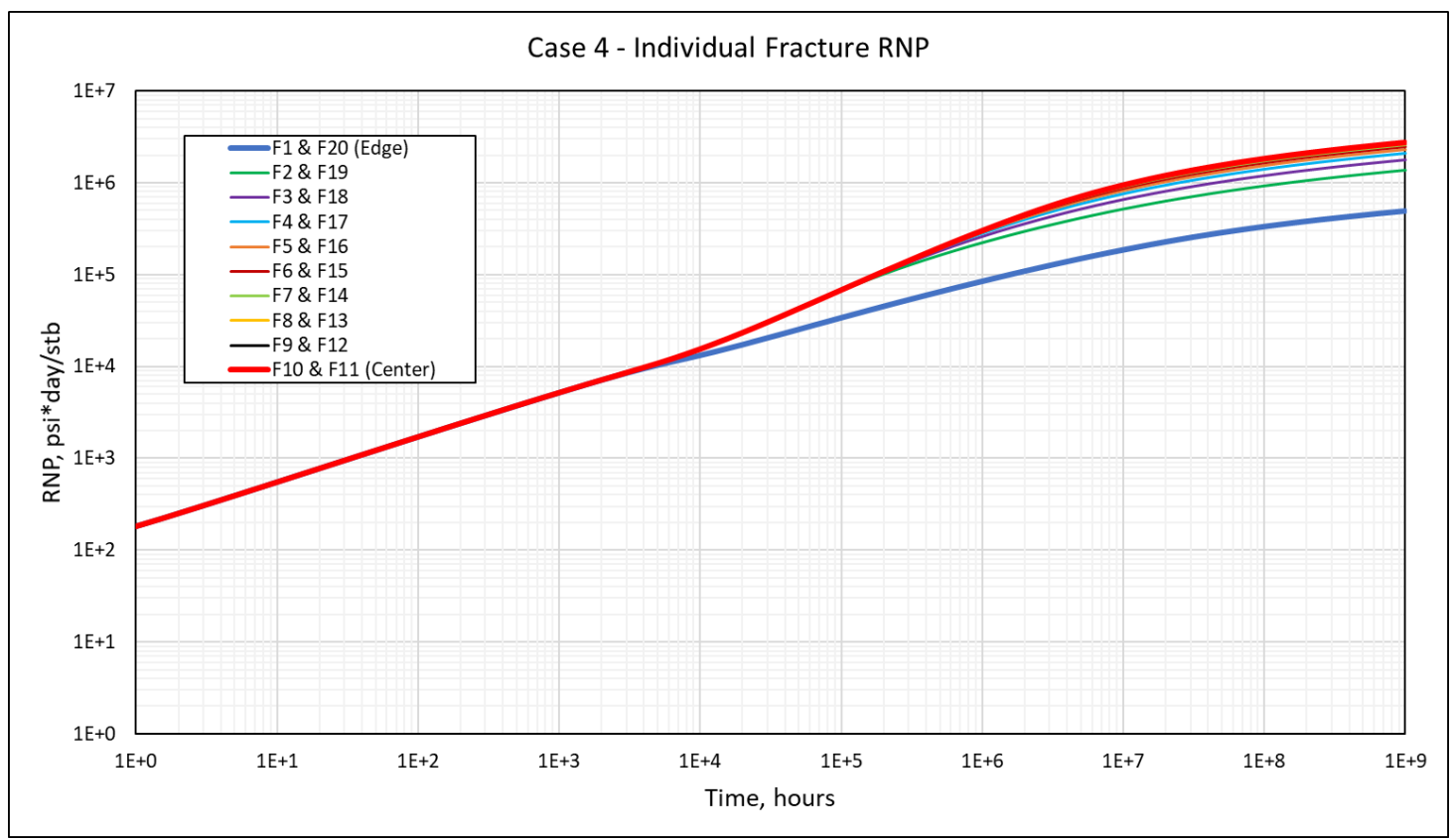

Figure 48 - Case 4 - Individual Fracture RNP

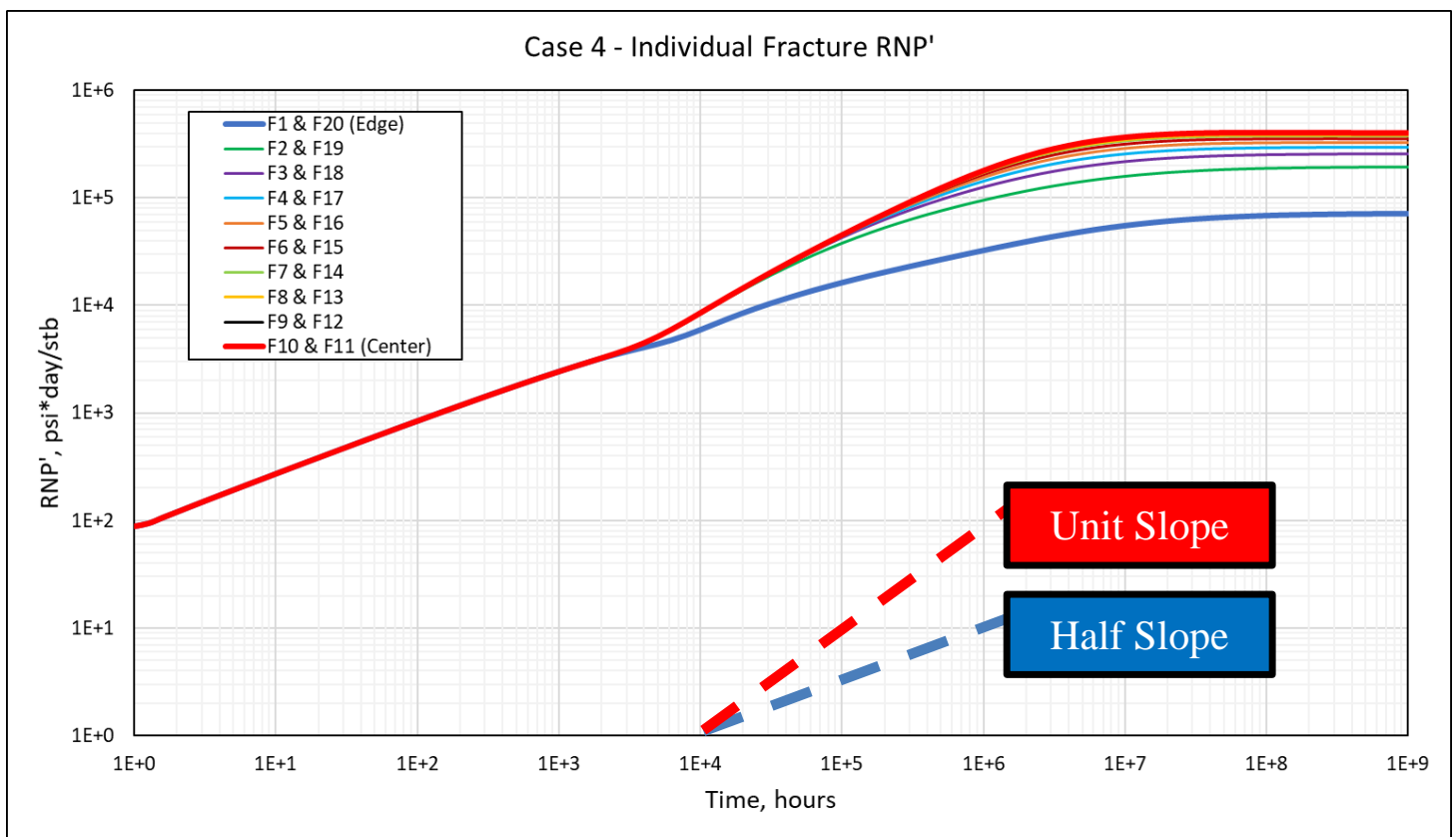

Figure 49 - Case 4 - Individual Fracture RNP' 


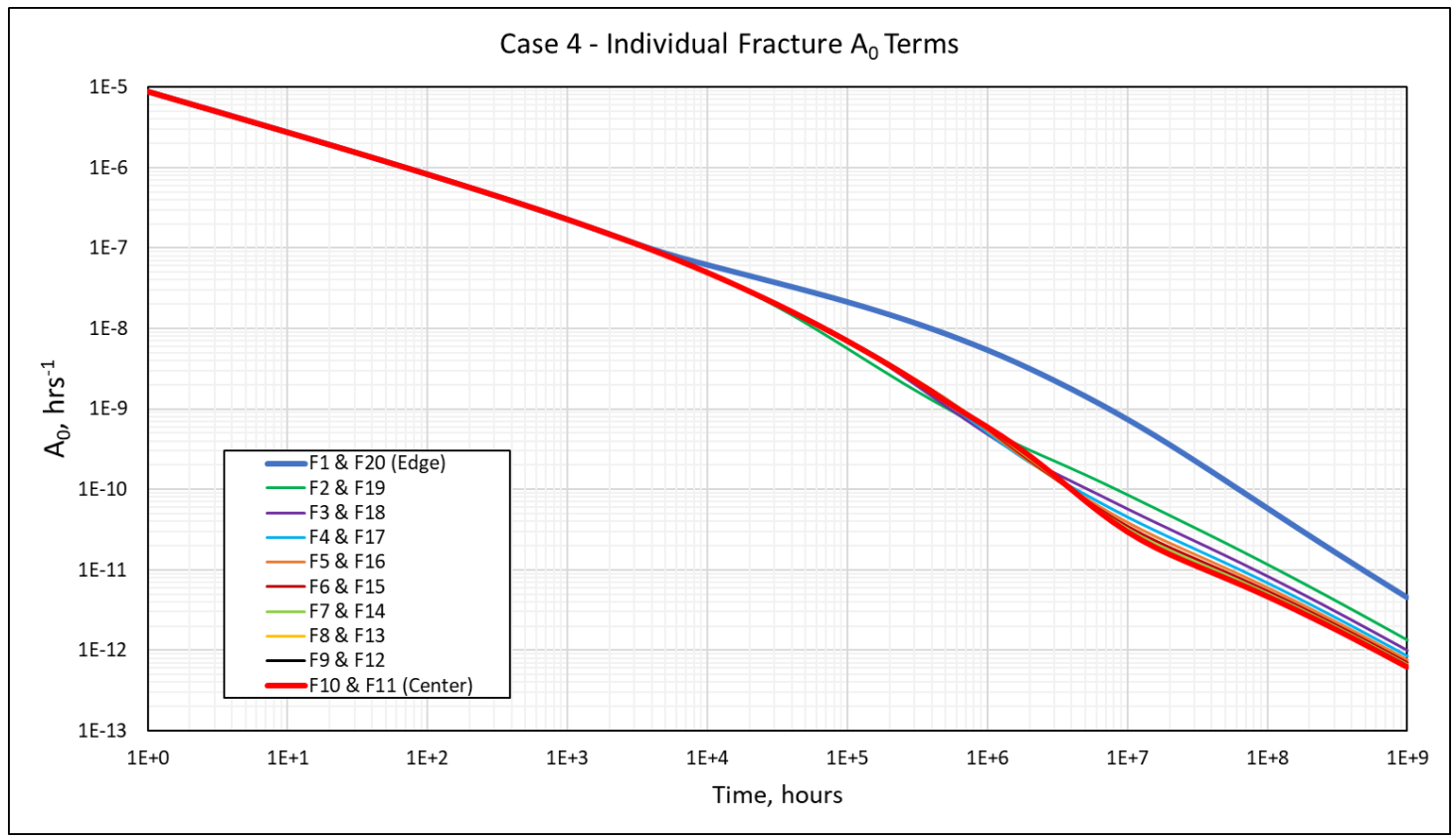

Figure 50 - Case 4 - Individual Fracture Ao Terms

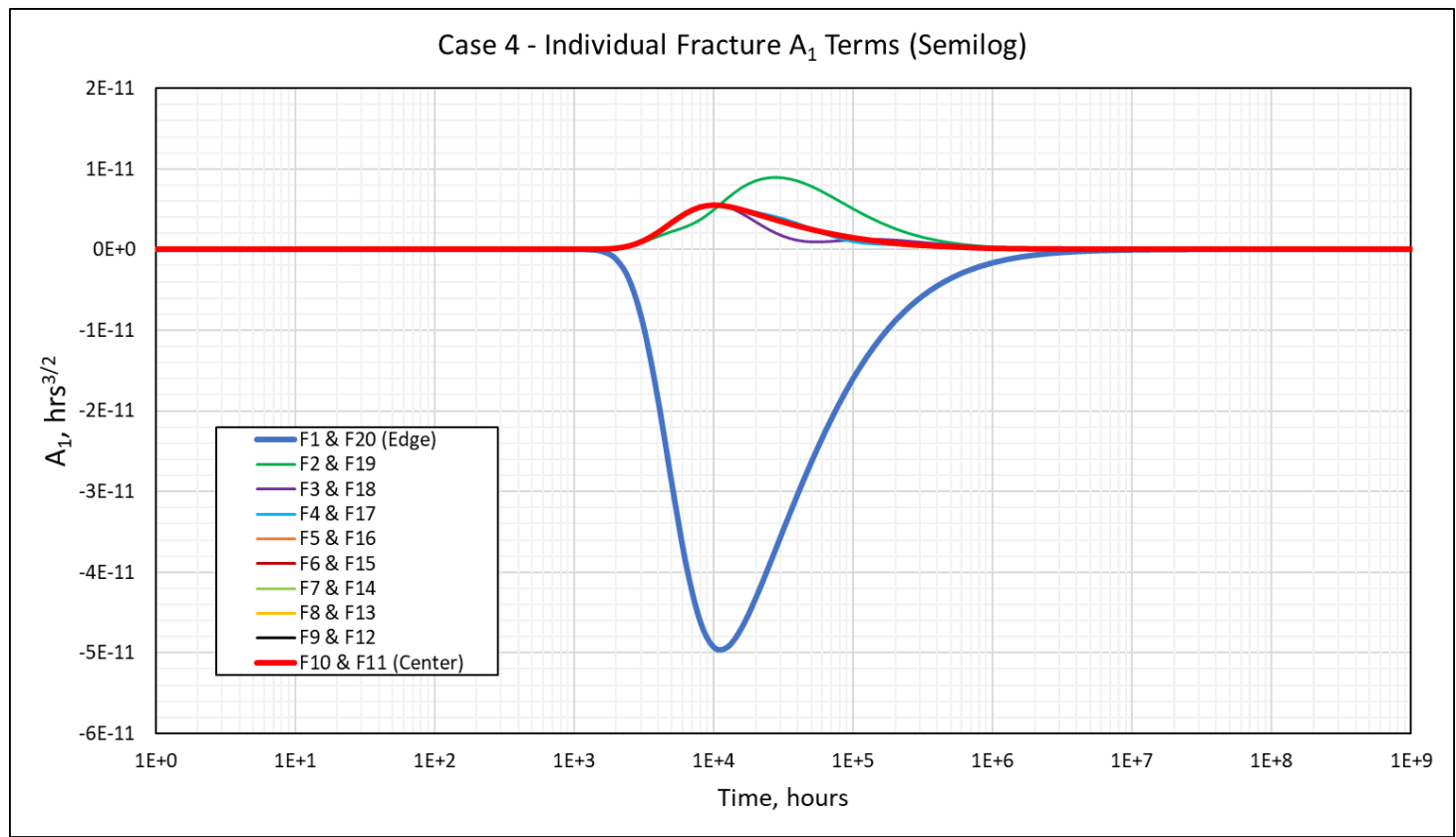

Figure 51 - Case 4 - Individual Fracture A1 Term 


\section{Case 5 - Constant BHP IALF $\left(A_{1}\right)$ - Results}

Our constant-BHP formulations did not achieve the same levels of success we saw with our constant-rate models. Nonetheless, with this study we wanted to test and document the results of using the asymptotic approximation of the diffusivity equation in conjunction with the principle of superposition in space. Case 5's analytic solution managed to do one thing correctly - it matched both the early time linear flow period and late time channel linear flow period of the reference model (Figure 52).

The analytic solution matches the reference model at early time (preinterference) because the fractures all act independently, and our whole-well response reflects the equally-shared proportions of flux to each fracture (Figure 53) which is seen as linear flow on a by-fracture basis (Figure 54, Figure 55). Once interference begins, the analytic model fails to match the reference model. The reference model's interference signature when plotted against time shows a unique signature - a sharp rise in RNP and a non-monotonic increase in RNP' that lasts approximately two decades. The analytic model fails to reproduce this non-monotonic signature and appears more like its constant-rate sibling shown in Figure 33. As we progress past the interference period, the rate allocation to the inner fractures continues to decrease much more quickly than the rate allocation to the edge fractures (Figure 53). The whole-well response begins to more closely reflect the edge fracture linear flow behavior as the fraction of flow to the edge fractures increases. 
As with any single-term approximation, the one term we use will dominate. In this case that will be the $A_{1}$ term. $A_{1}$ shows an equal monotonic decrease for all fractures until fracture interference begins, and then the $A_{1}$ terms separate (Figure 56).

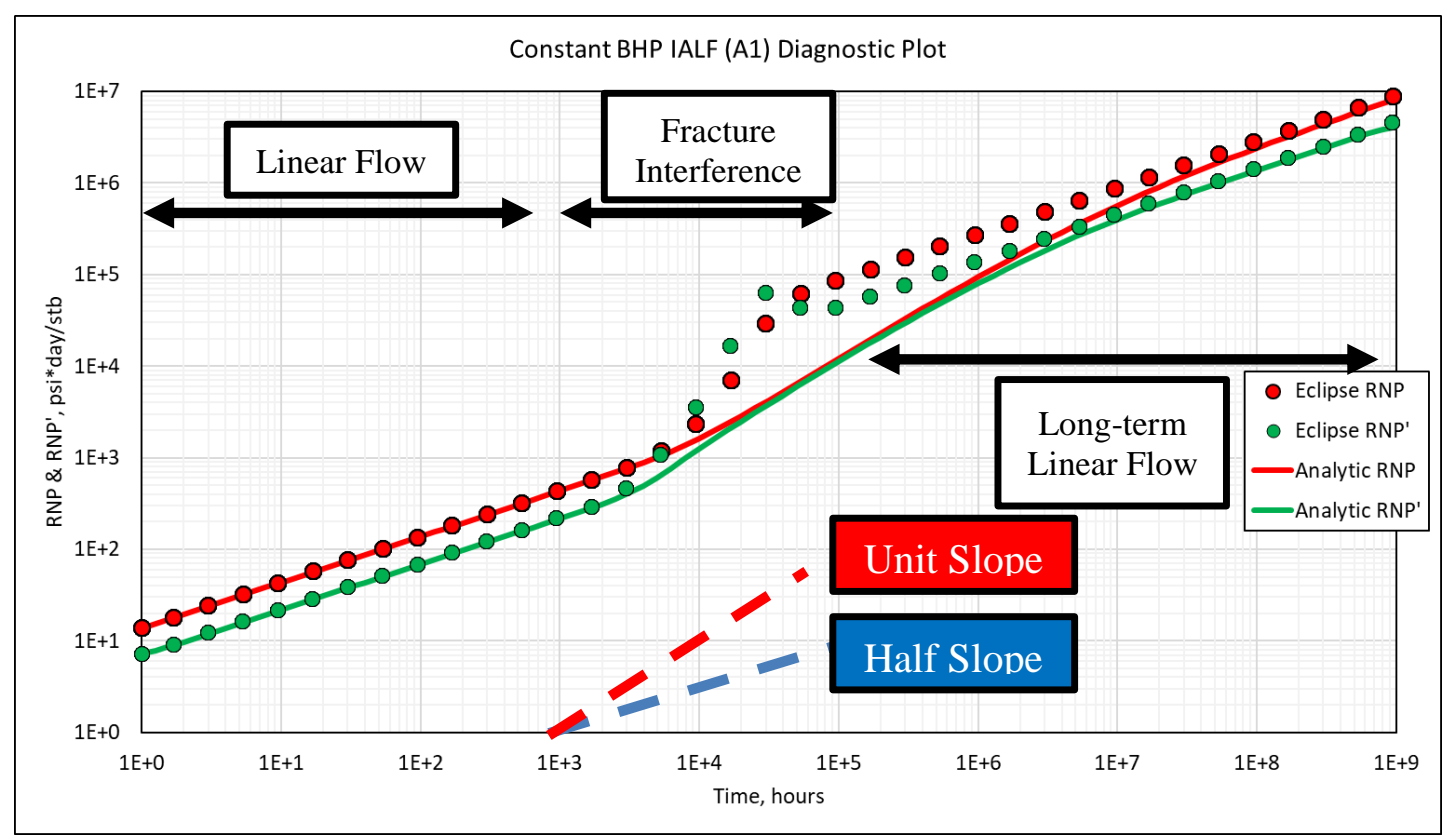

Figure 52 - Case 5 Diagnostic Plot Comparison with Reference Mode 


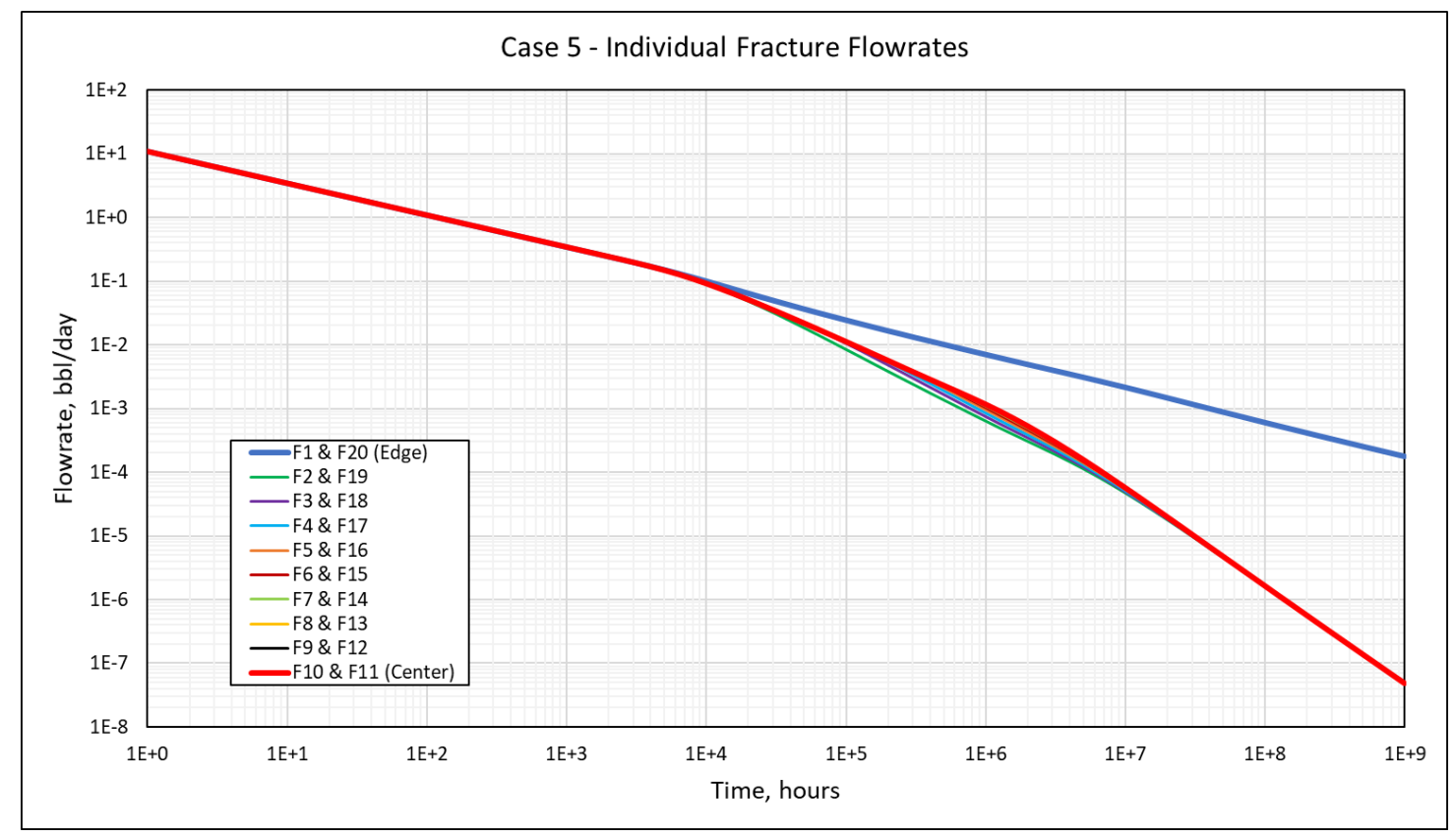

Figure 53 - Case 5 - Individual Fractures Flowrates

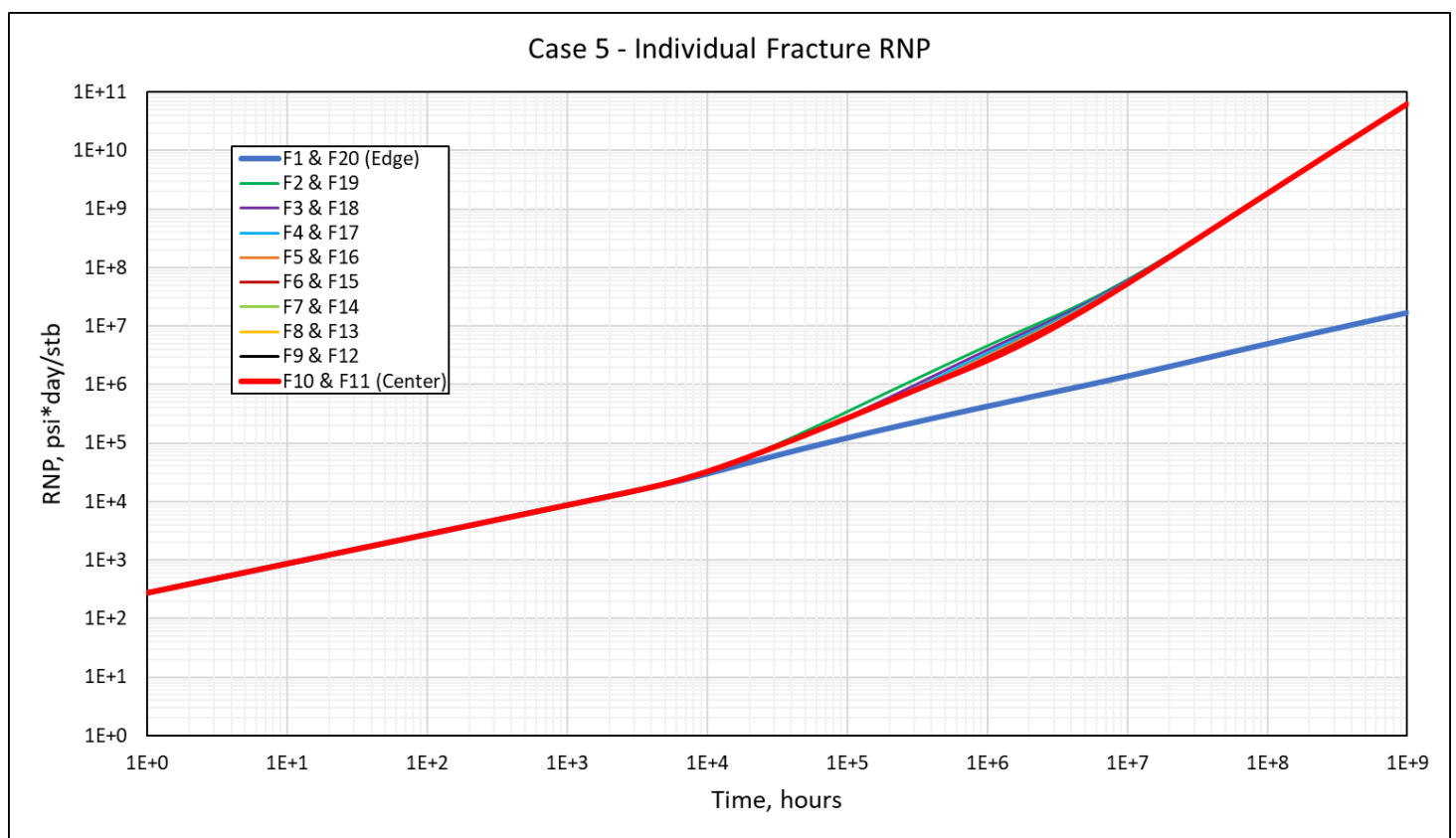

Figure 54 - Case 5 - Individual Fracture RNP 


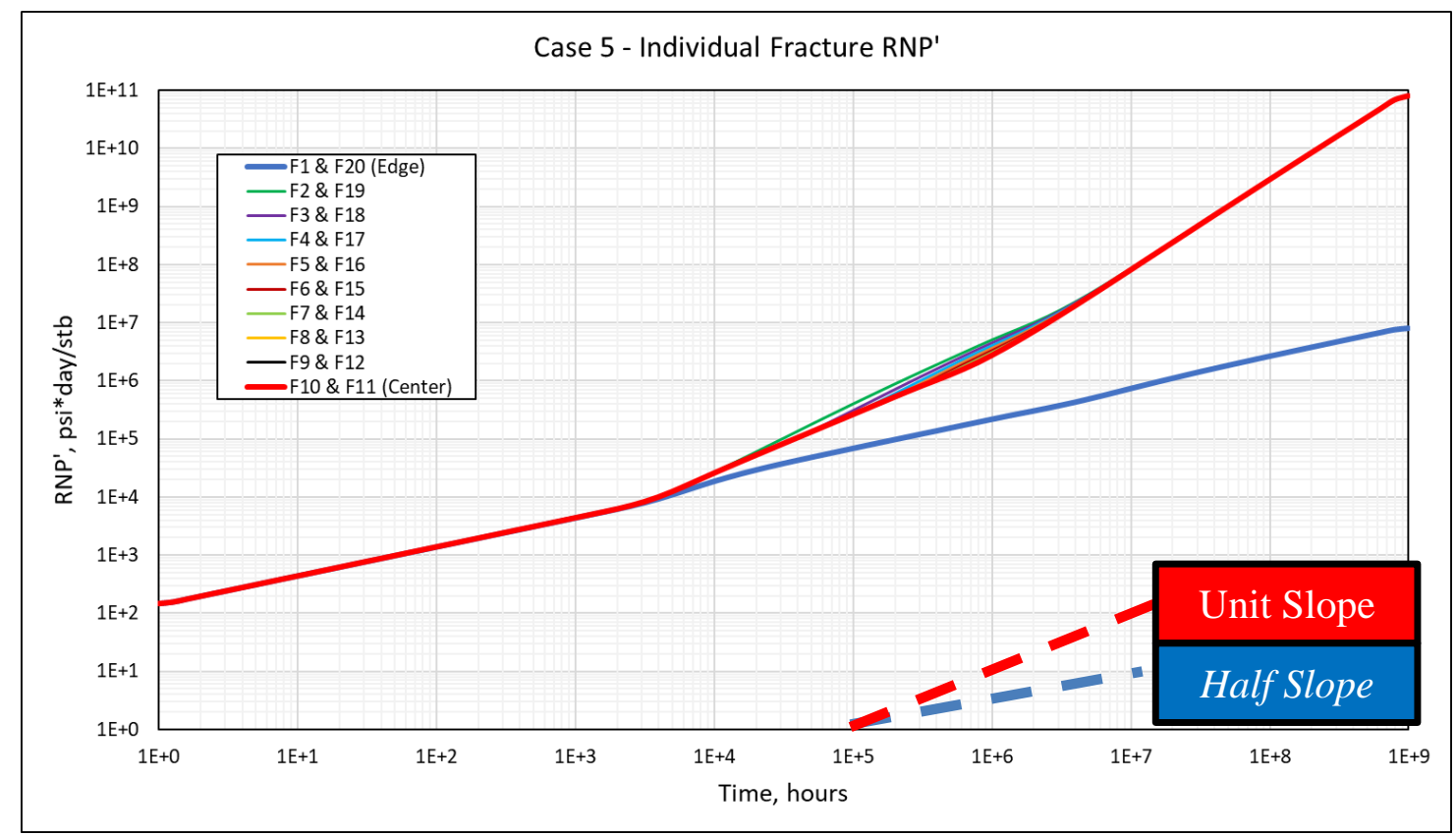

Figure 55 - Case 5 - Individual Fracture RNP'

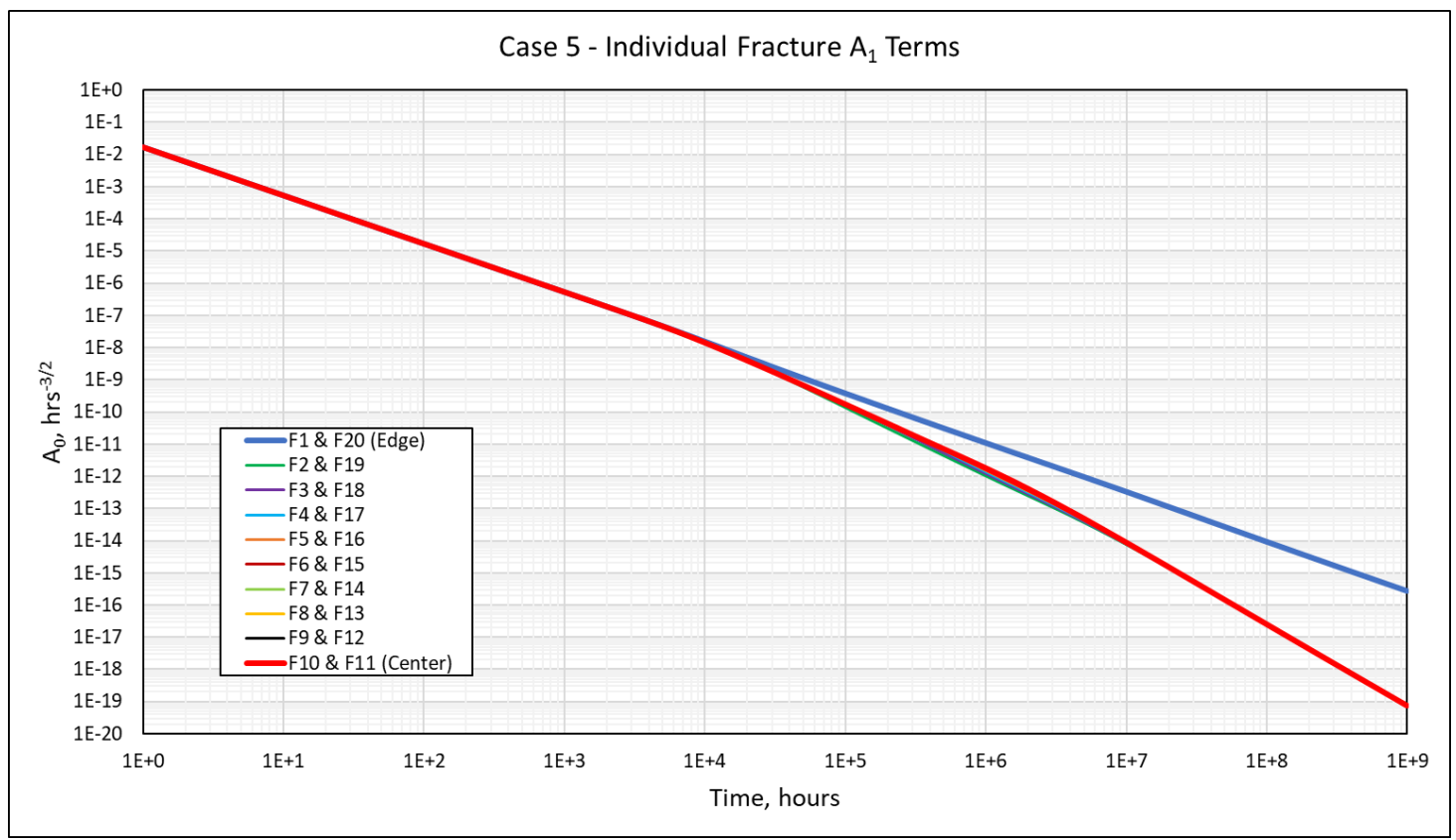

Figure 56 - Case 5 - Individual Fracture A1 Terms 
Case 6 - Constant BHP IALF (Ao and $\left.A_{1}\right)$ - Results

Case 6, the two-term IALF case, performed much better than its 1-term relative. It matched the early and late time linear flow periods and managed to approximate the fracture interference period behavior much more accurately but still fails to exactly match our reference solution (Figure 57).

As with previous cases, the early well flow behavior is driven by an equallydistributed flux from all fractures that appears as linear flow on both the whole-well diagnostic plot and the individual fracture RNP and RNP' curves (Figure 59 and Figure 60). Fracture interference appears on the analytic model with a signature similar the reference model's, but the analytic model response still holds fully monotonic, whereas the reference solution's interference signature shows up as a non-monotonic RNP' curve.

The unknown function, $A_{1}$, drives this solution, showing the same monotonic decrease through time for all fractures, with a split between the edge fracture and inner fracture responses when fracture interference begins (Figure 62). The $A_{0}$ term appears to influence only the fracture interference period, causing a decrease in the flux to both the inner and edge fractures, but more heavily affecting the inner fractures (Figure 61). 


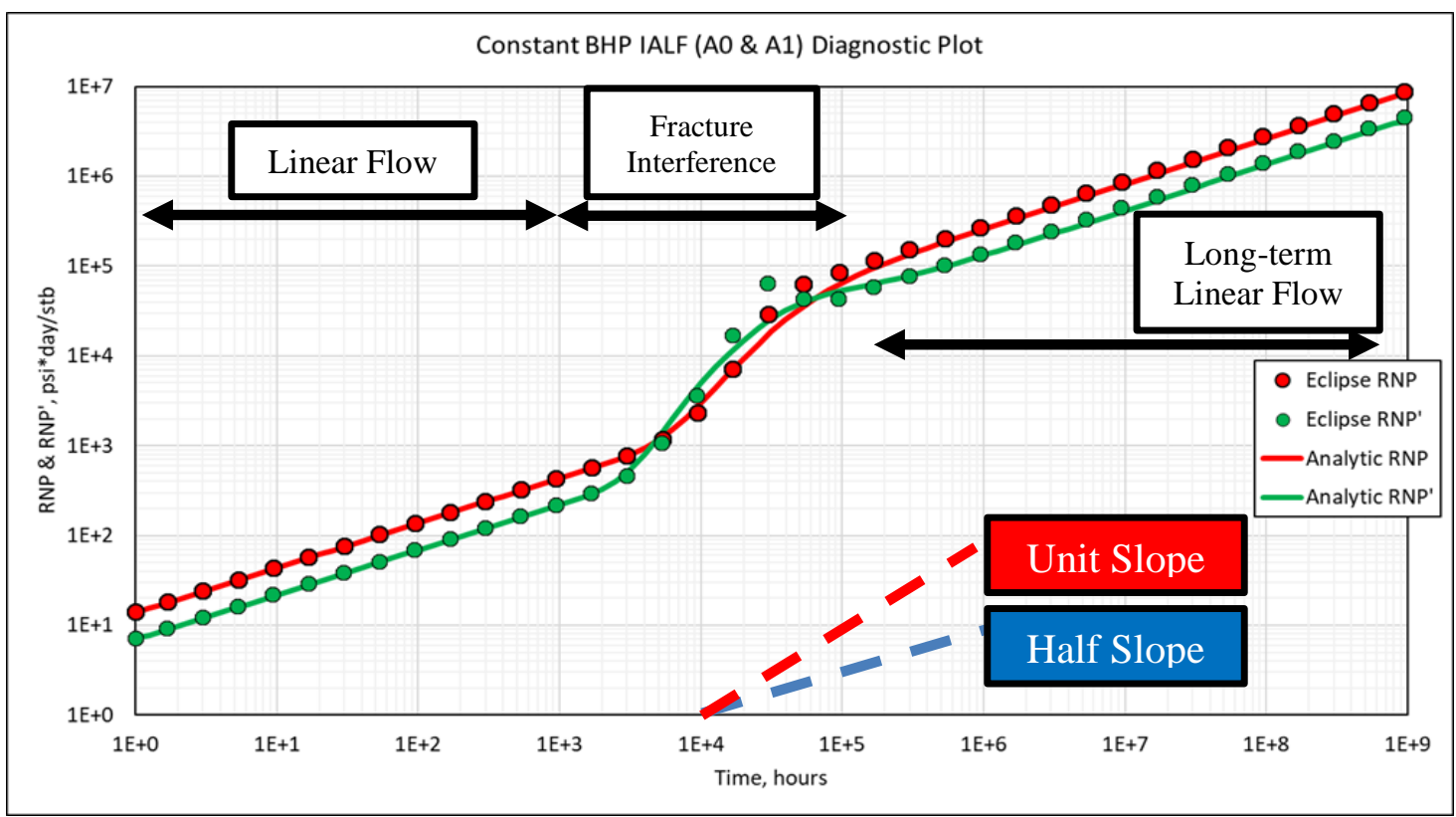

Figure 57 - Case 6 Diagnostic Plot Comparison with Reference Model

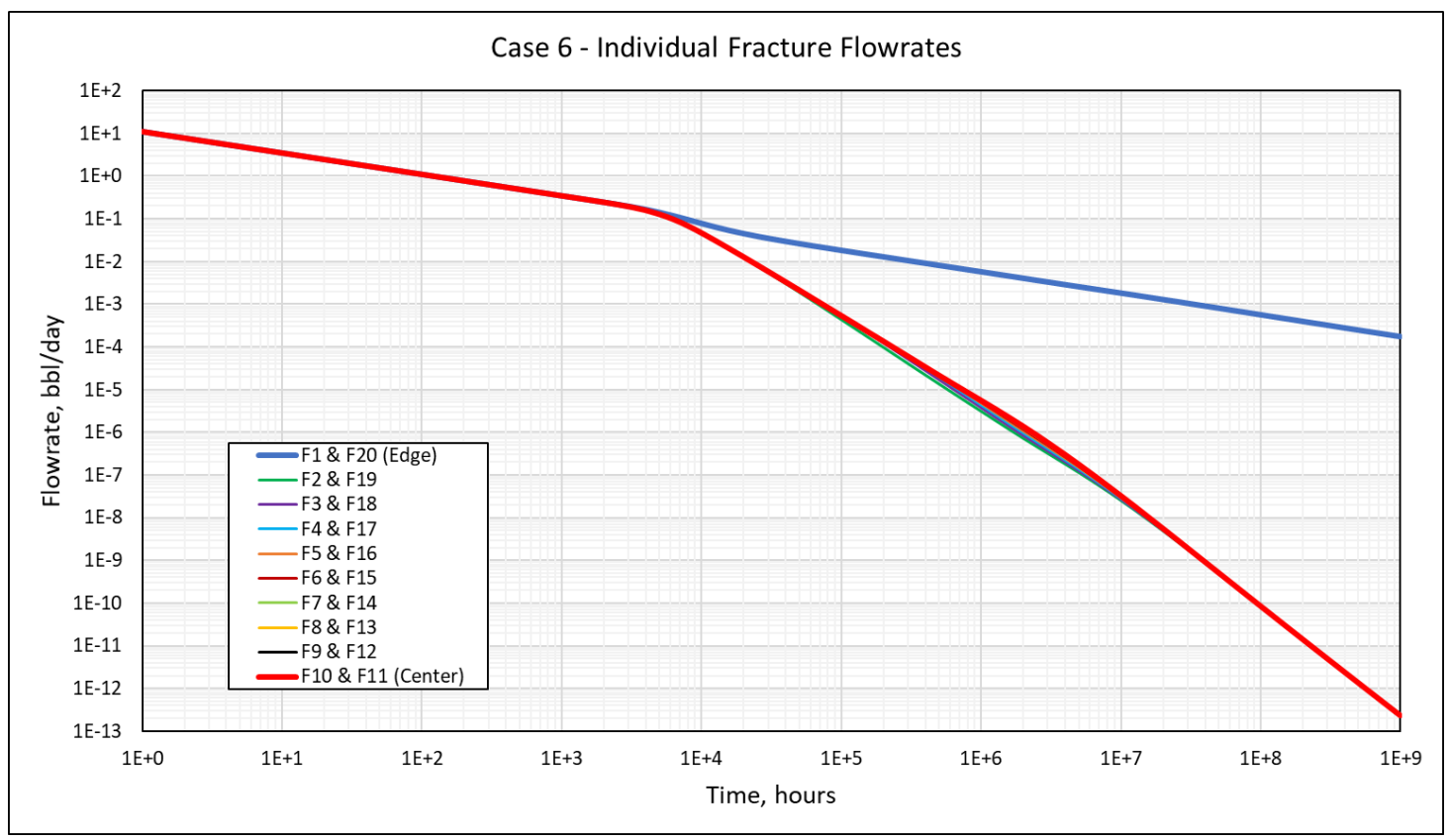

Figure 58 - Case 6 - Individual Fractures Flowrates 


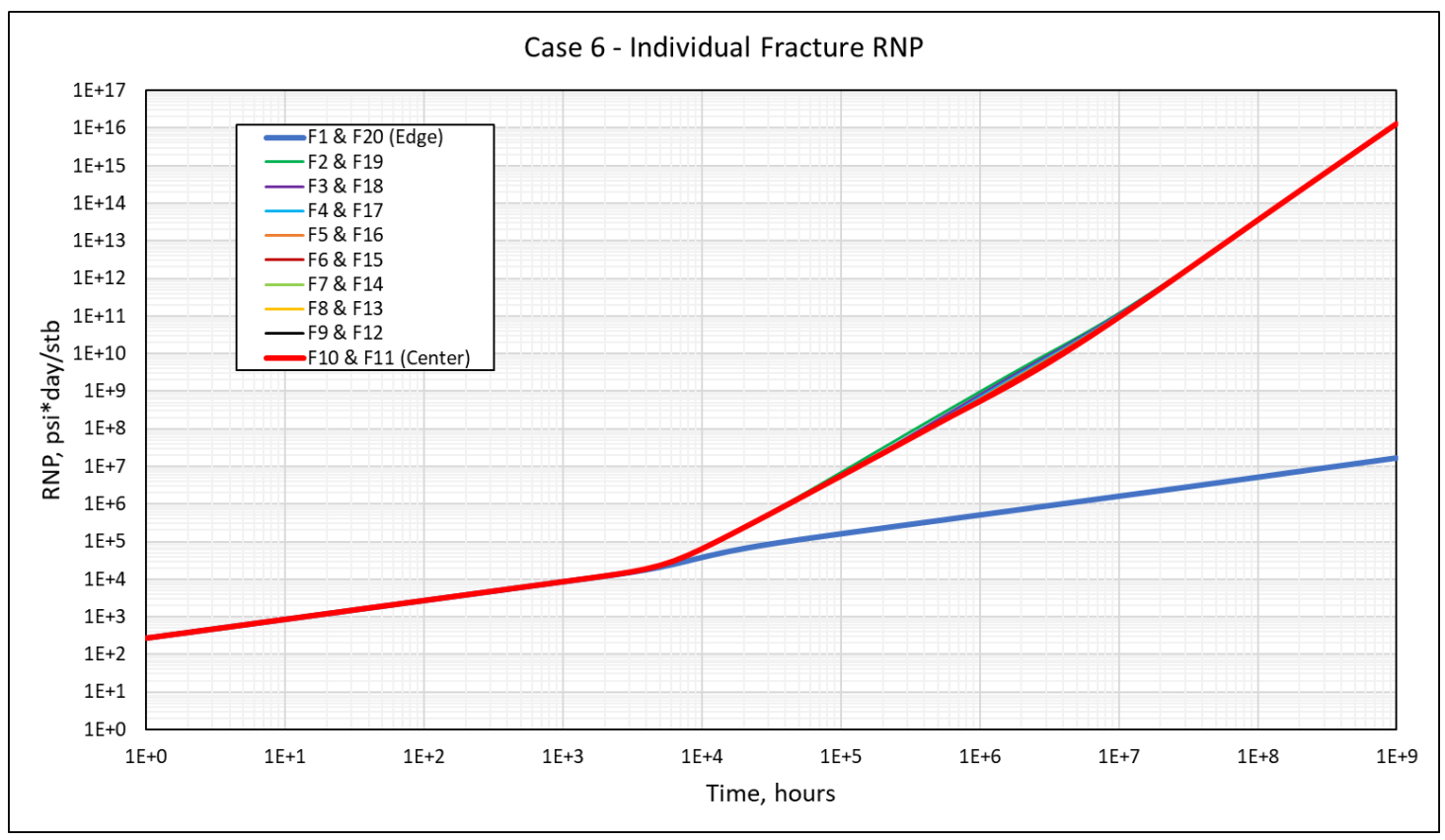

Figure 59 - Case 6 - Individual Fracture RNP

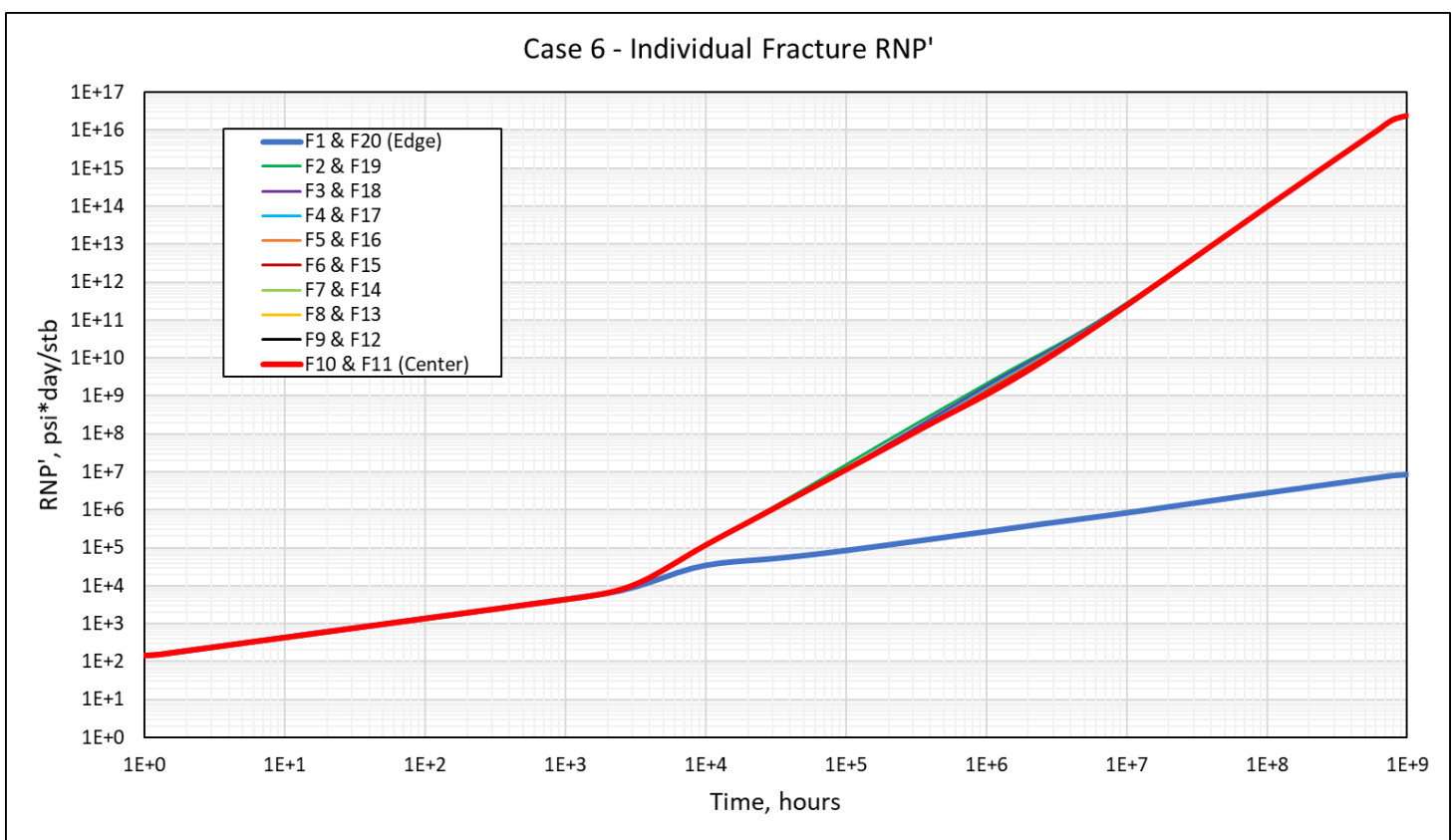

Figure 60 - Case 6 - Individual Fracture RNP' 


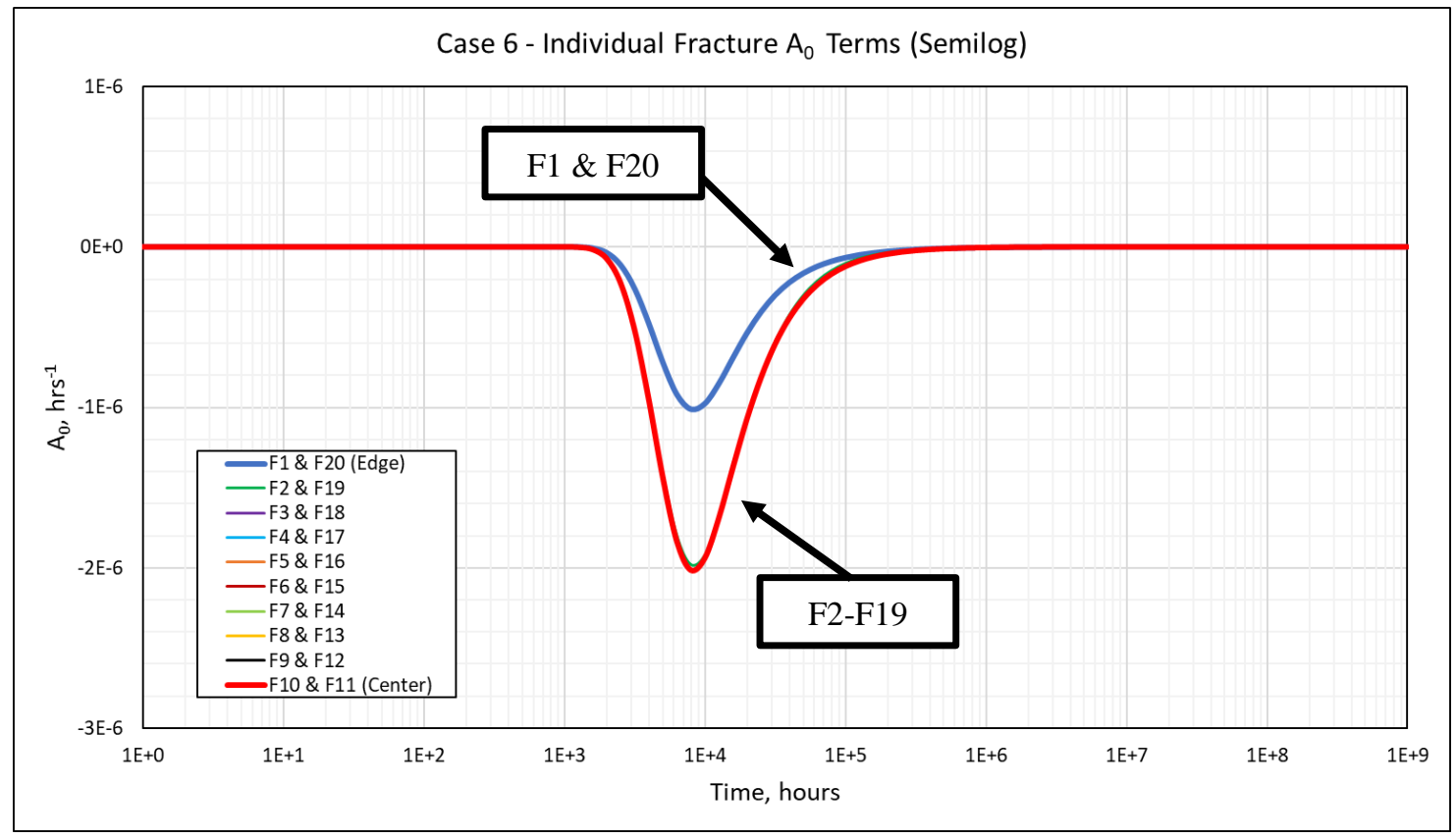

Figure 61 - Case 6 - Individual Fracture Ao Terms

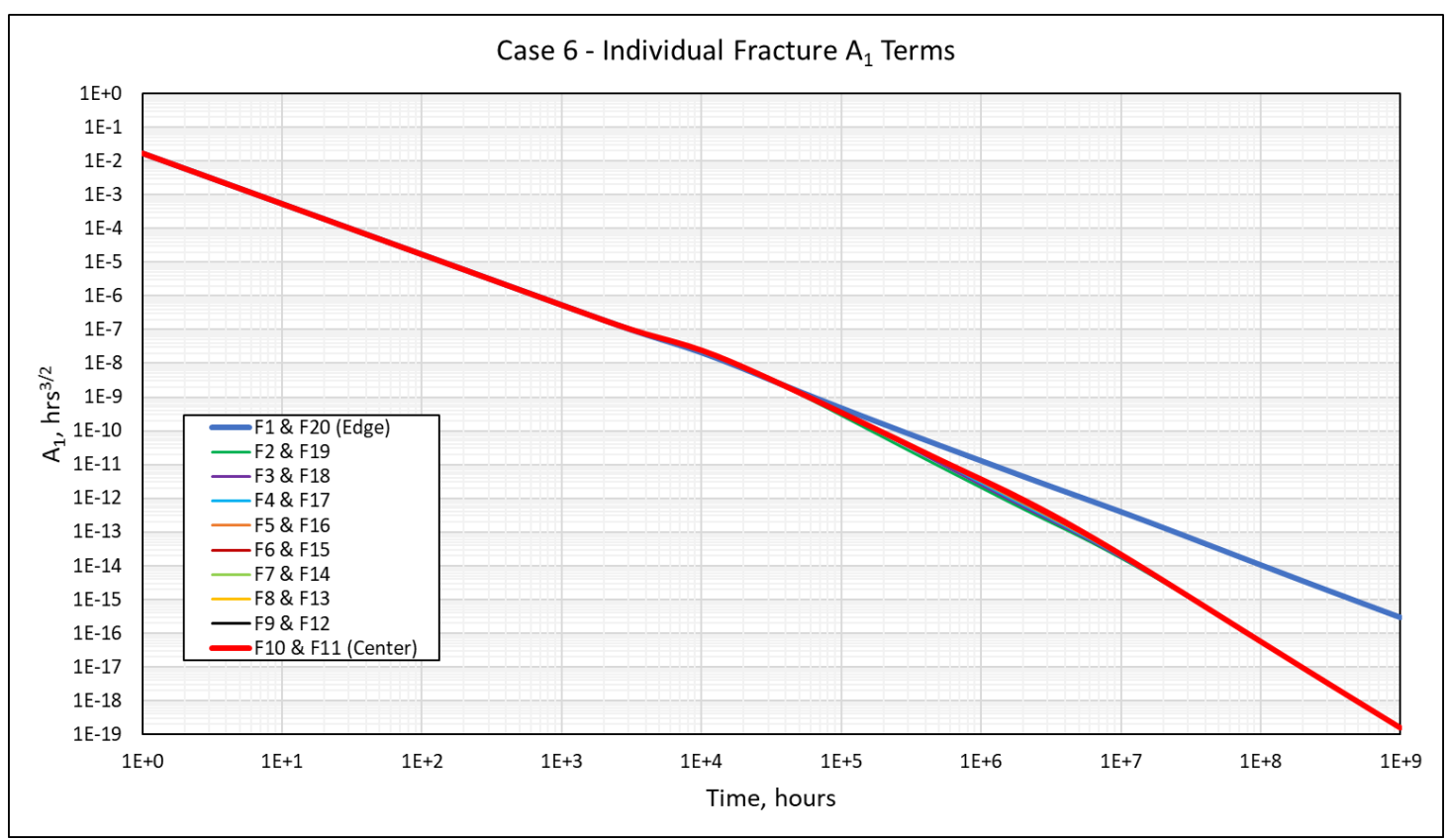

Figure 62 - Case 6 - Individual Fracture A1 Terms 


\section{Case 7 - Constant BHP IAPF $\left(A_{1}\right)-$ Results}

The results from Case 7 were similar in nature to those from Case 5. The analytic model matches the reference model during the early time linear flow period, and the latetime transition to radial flow, but fails to match the fracture interference behavior (Figure 63). As with the 1-dimensional constant-BHP cases, the 2-dimensional reference model shows non-monotonicity in its RNP' curve, and the analytic model is unable to match that.

Individual fracture flowrates (Figure 64) indicate that fractures act independently during early time linear flow, showing a constant rate decay until the onset of fracture interference. After interference begins, this decay accelerates for central fractures and remains mostly constant for the edge fractures. At late time, the rates for all fractures stabilize as they begin to flow collectively.

RNP and RNP' behavior (Figure 65 and Figure 66) at the individual fracture level shows linear flow for all fracture during early time, and then a divergence upon the onset of fracture interference. Central fractures' RNP' curves indicate an apparent boundary, and the edge fractures seem relatively undisturbed. As time progresses, fractures progressively stabilize as they receive the pressure "signal" from all their neighbors. Eventually, collective radial flow develops as we see stabilization to a slope of zero for all fractures at very late time. 


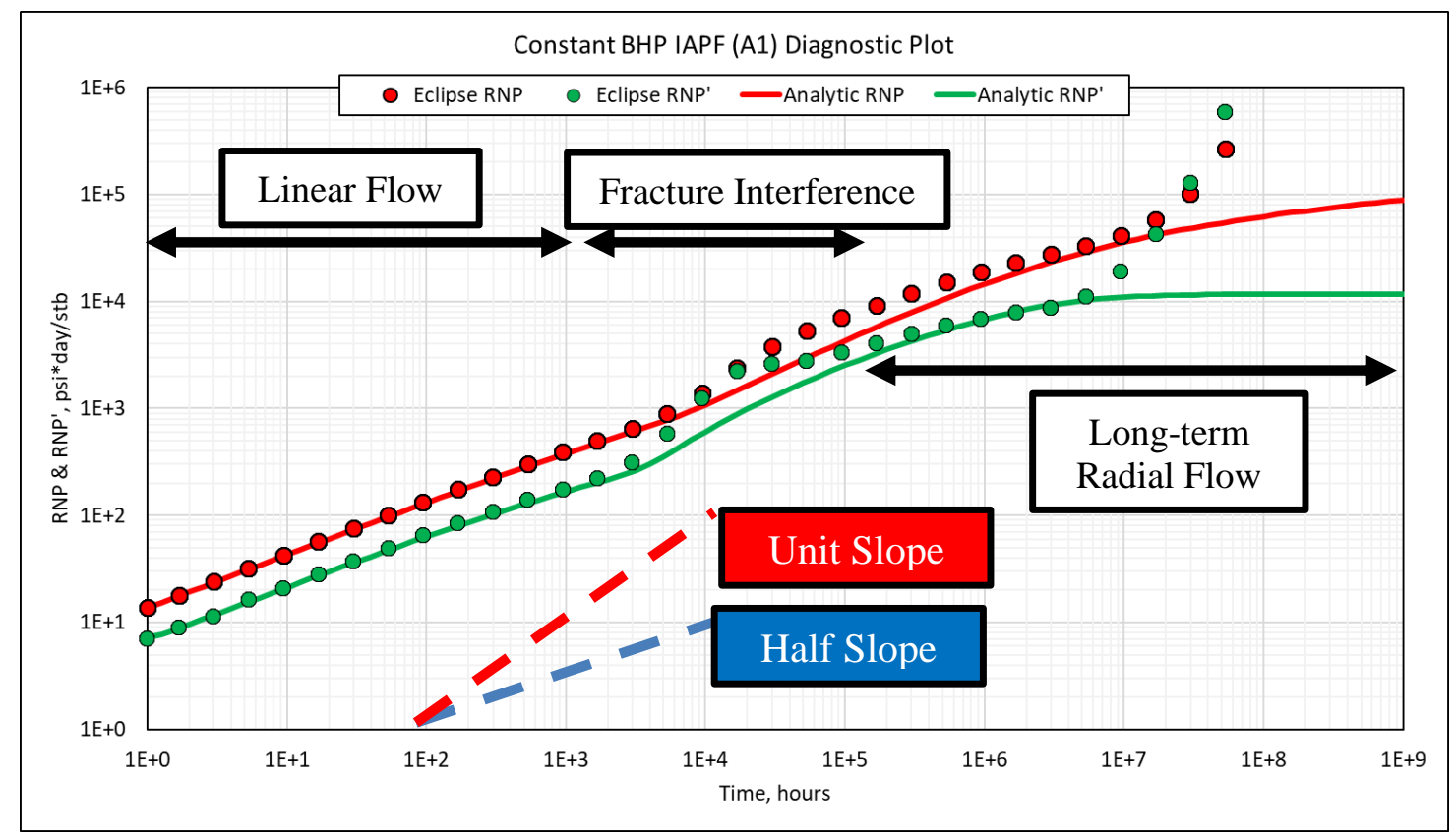

Figure 63 - Case 7 Diagnostic Plot Comparison with Reference Model

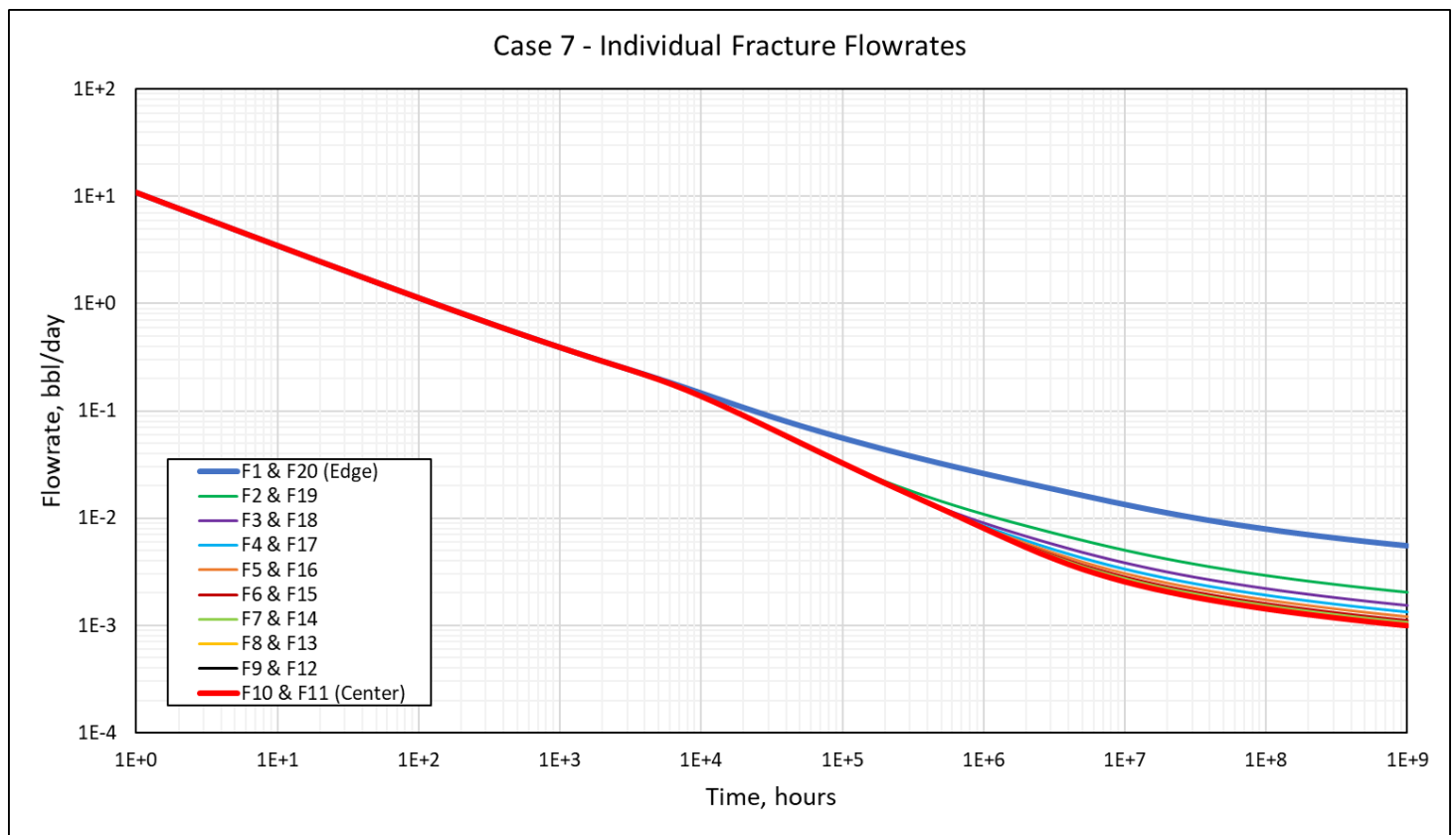

Figure 64 - Case 7 - Individual Fractures Flowrates 


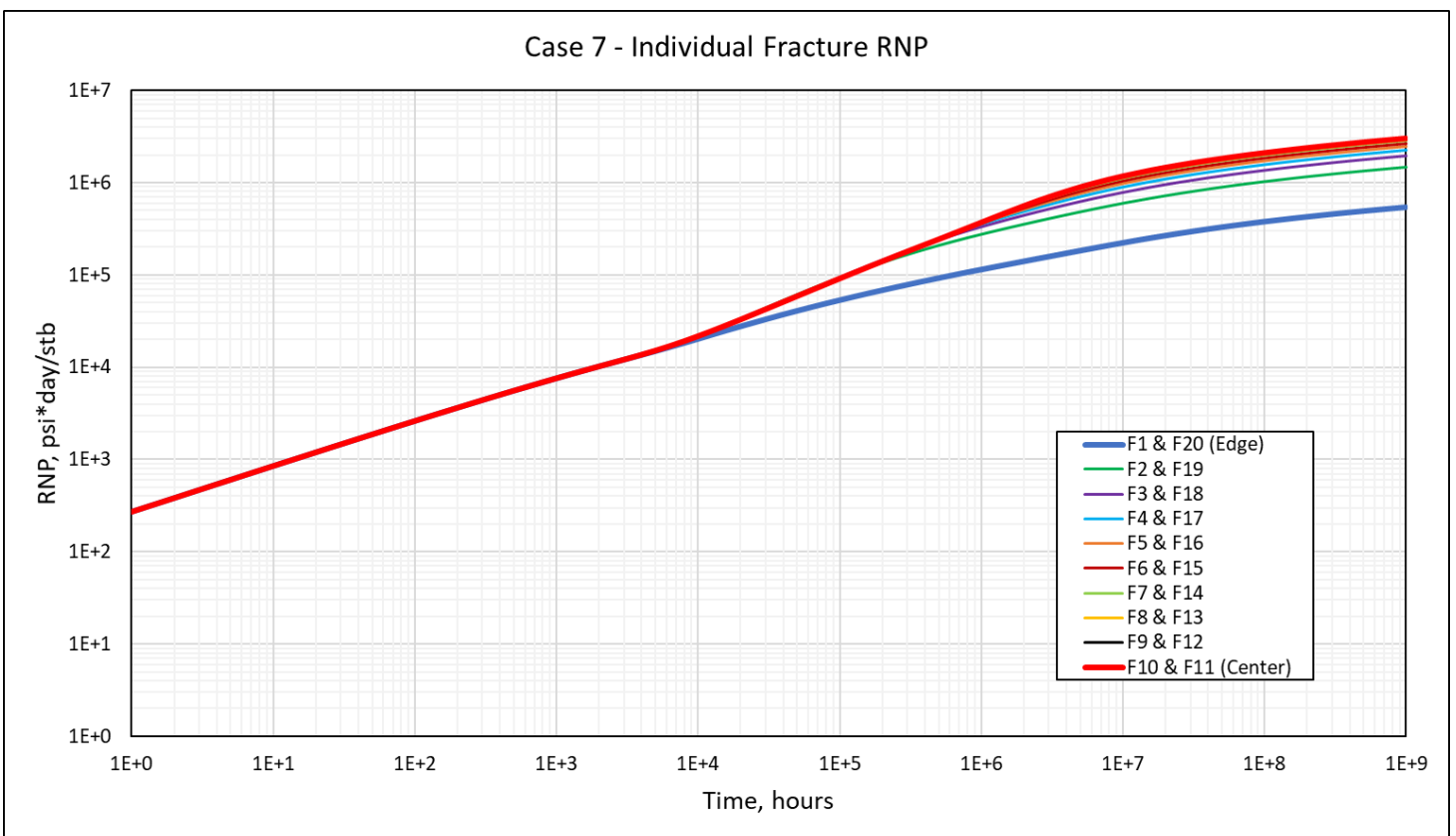

Figure 65 - Case 7 - Individual Fracture RNP

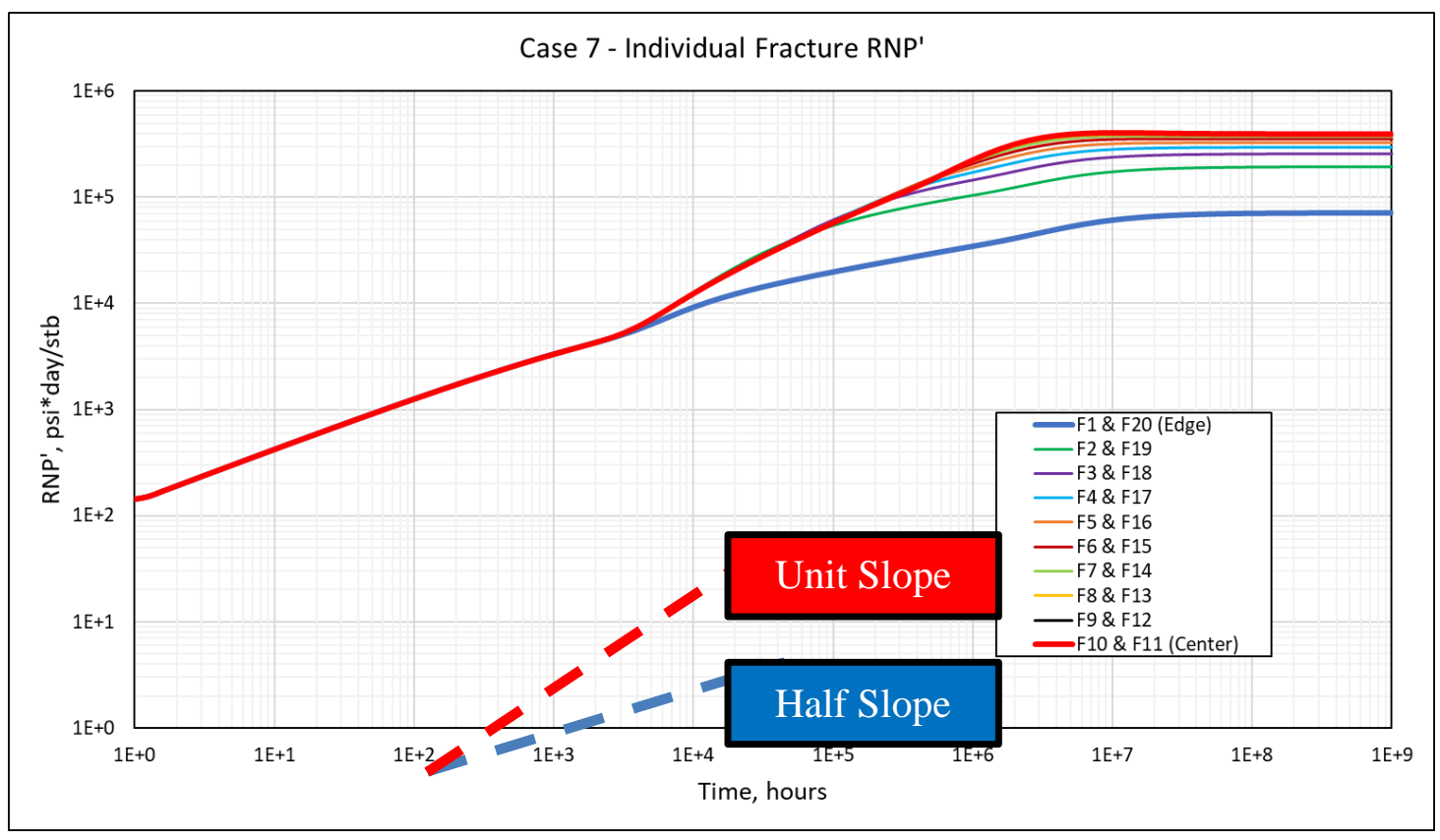

Figure 66 - Case 7 - Individual Fracture RNP' 


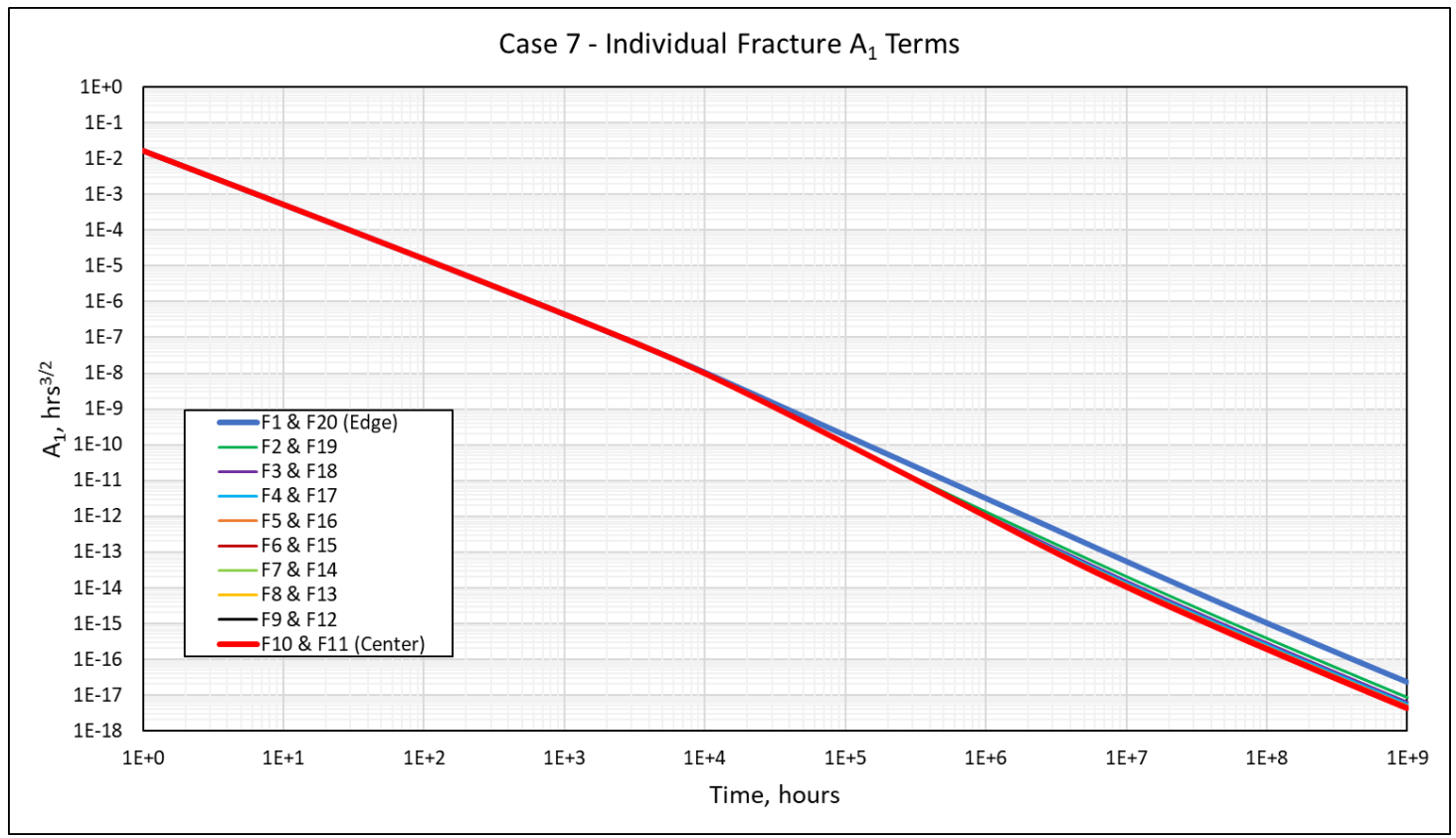

Figure 67 - Case 7 - Individual Fracture A1 Terms 


\section{Case 8 - Constant BHP IAPF (Ao and $\left.A_{1}\right)$}

Case 8's analytic solution performed very well relative to the reference model, failing only to capture the non-monotonicity displayed by the reference model's RNP' curve during fracture interference (Figure 68). It successfully models early time linear flow, a fracture interference period, and a late-time transition to infinite-acting radial flow.

At the individual fracture level, we see fracture act independently at early time, before diverging after the onset of fracture interference (Figure 69). Central fractures see a greater decay in their rates than the edge fractures, but all fractures eventually stabilize at late time as they begin to act collectively. The RNP and RNP' curves (Figure 70 and Figure 71) show all fractures operating independently under early linear flow until fracture interference. Once they begin communicating, all fractures see a boundary signature, but the signature is stronger for central fractures. As with rate, the diagnostics of individual fractures stabilize at late time, with all fractures eventually settling on a late-time collective radial flow behavior.

The $A_{1}$ term drives this solution (Figure 73), showing a monotonic decrease through all time, with a divergence between individual fracture terms after interference sets in. The $A_{0}$ term crops up during fracture interference (Figure 72) only, enabling the additional degree of freedom that makes this solution come so close to the reference. 


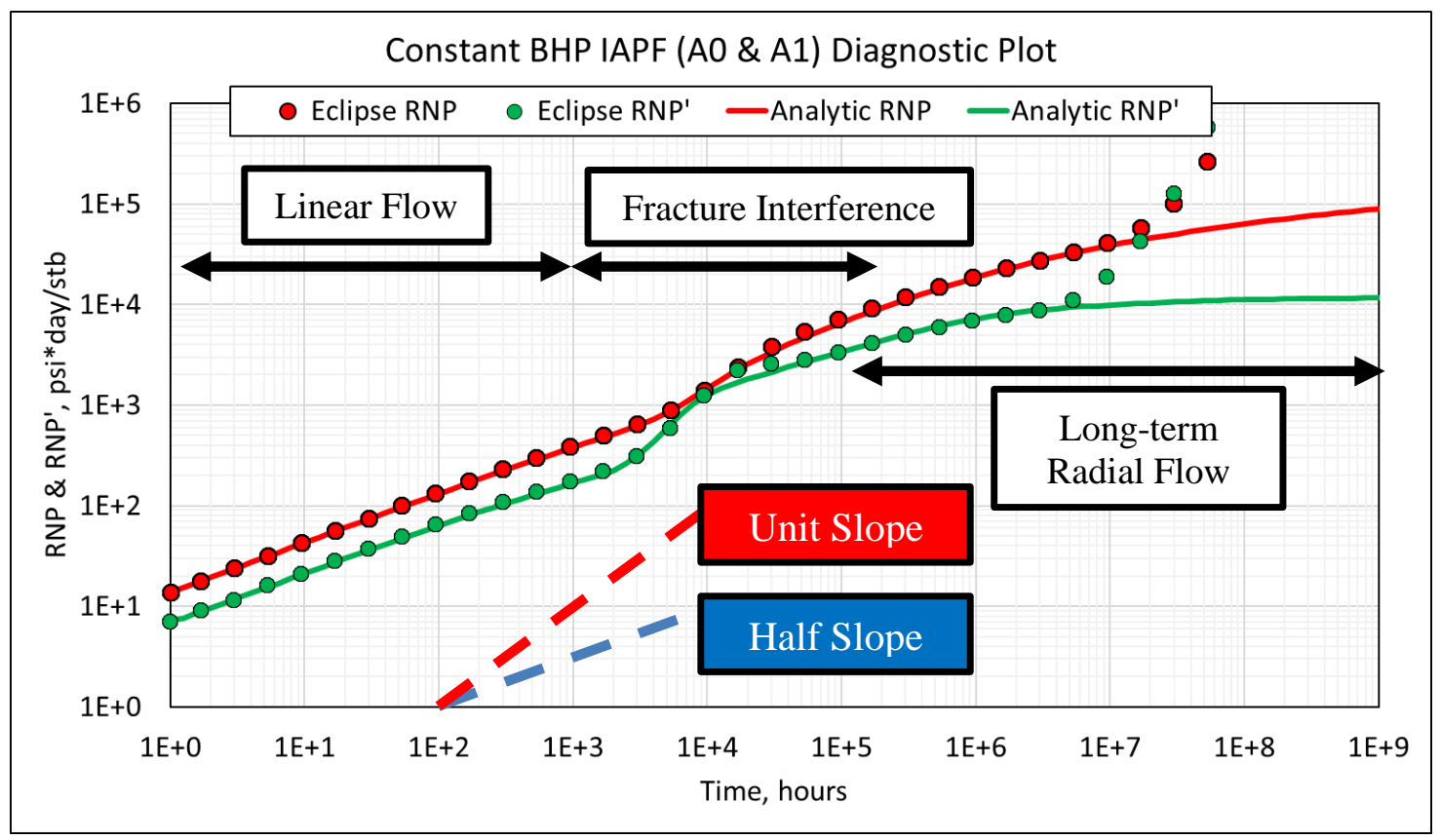

Figure 68 - Case 8 Diagnostic Plot Comparison with Reference Model (after Malone et al. (2019)). Copyright 2019, SPE. Reproduced with permission of SPE. Further reproduction prohibited without permission. 


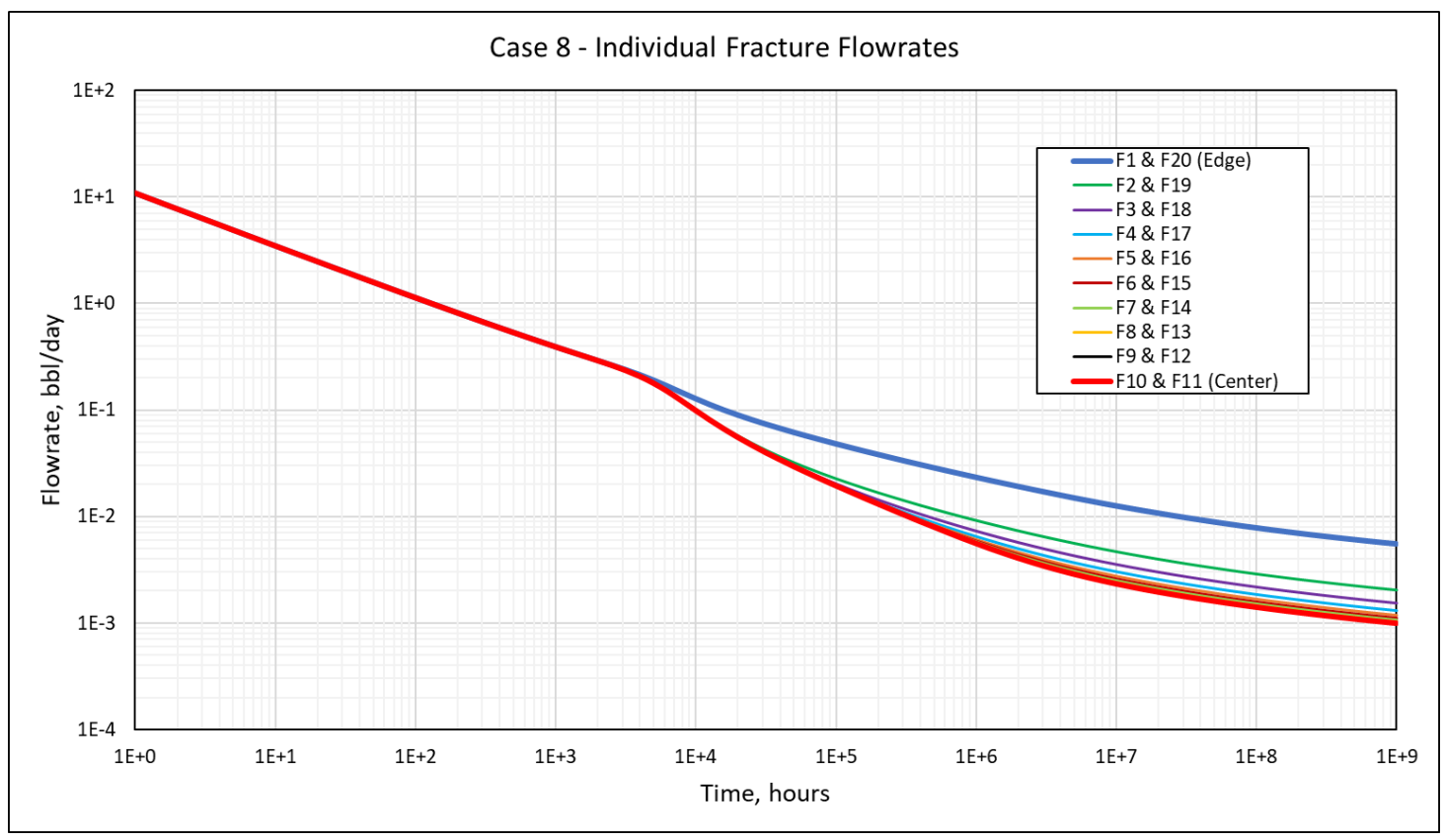

Figure 69 - Case 8 - Individual Fractures Flowrates

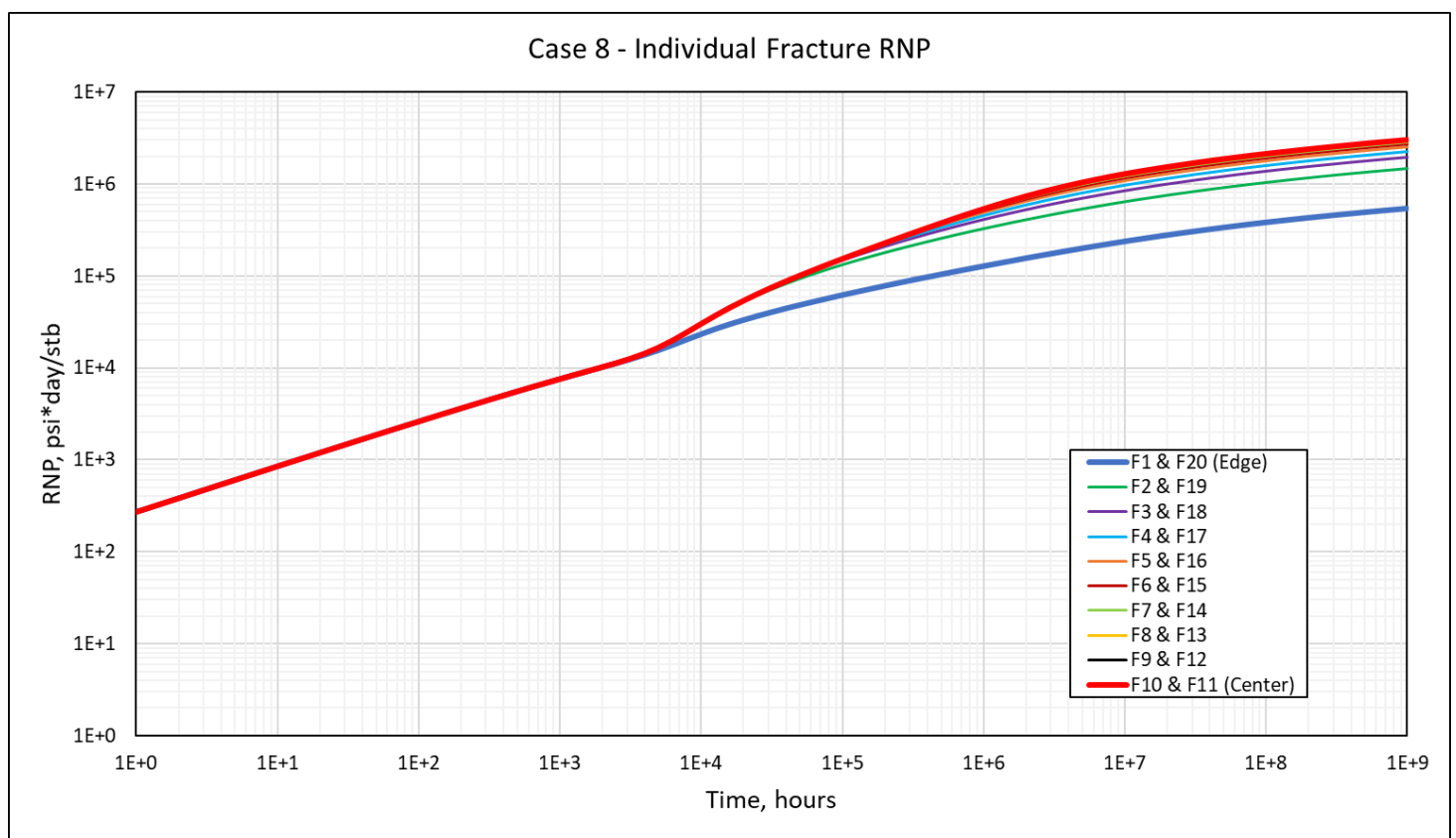

Figure 70 - Case 8 - Individual Fracture RNP 


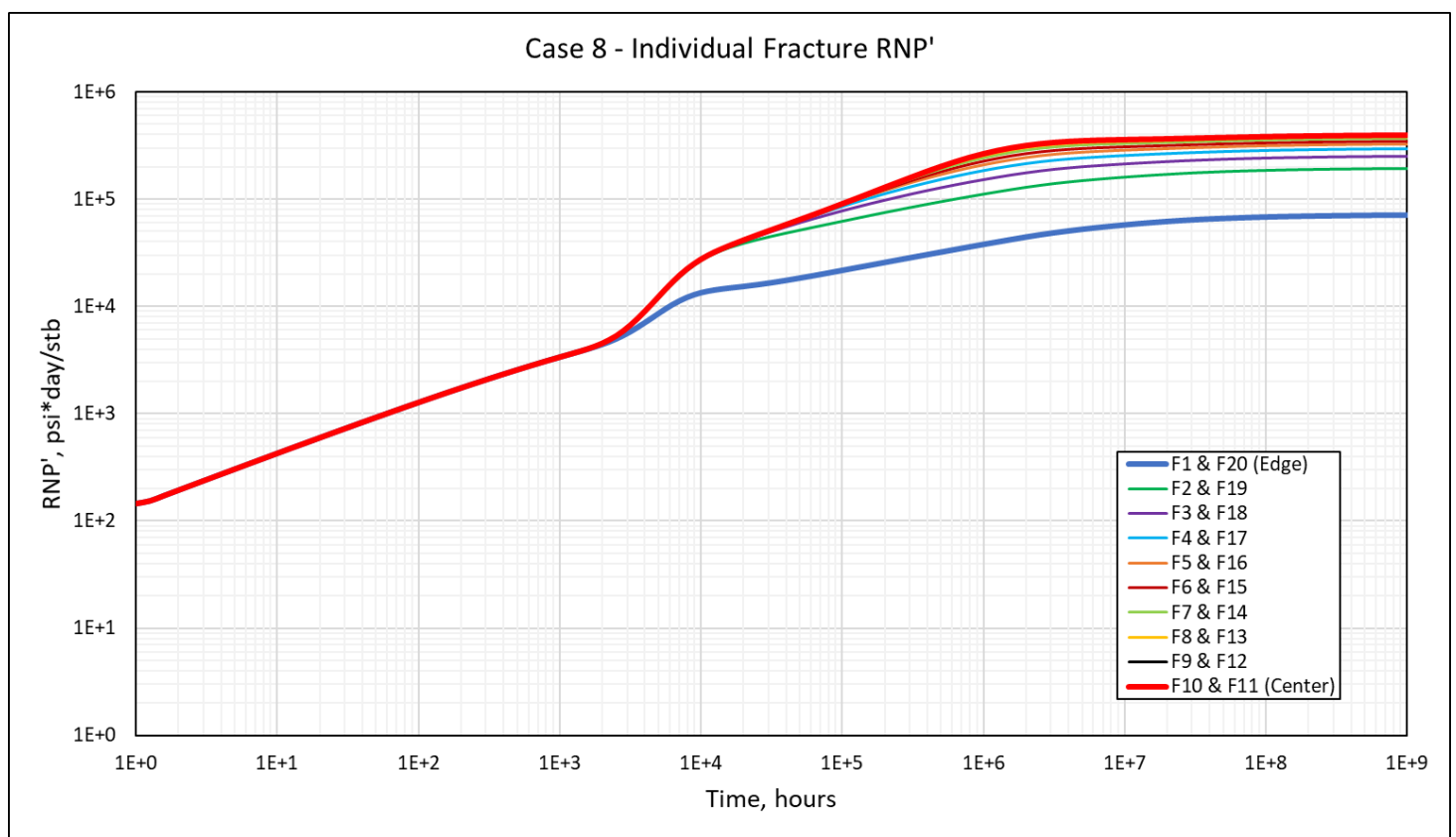

Figure 71 - Case 8 - Individual Fracture RNP'

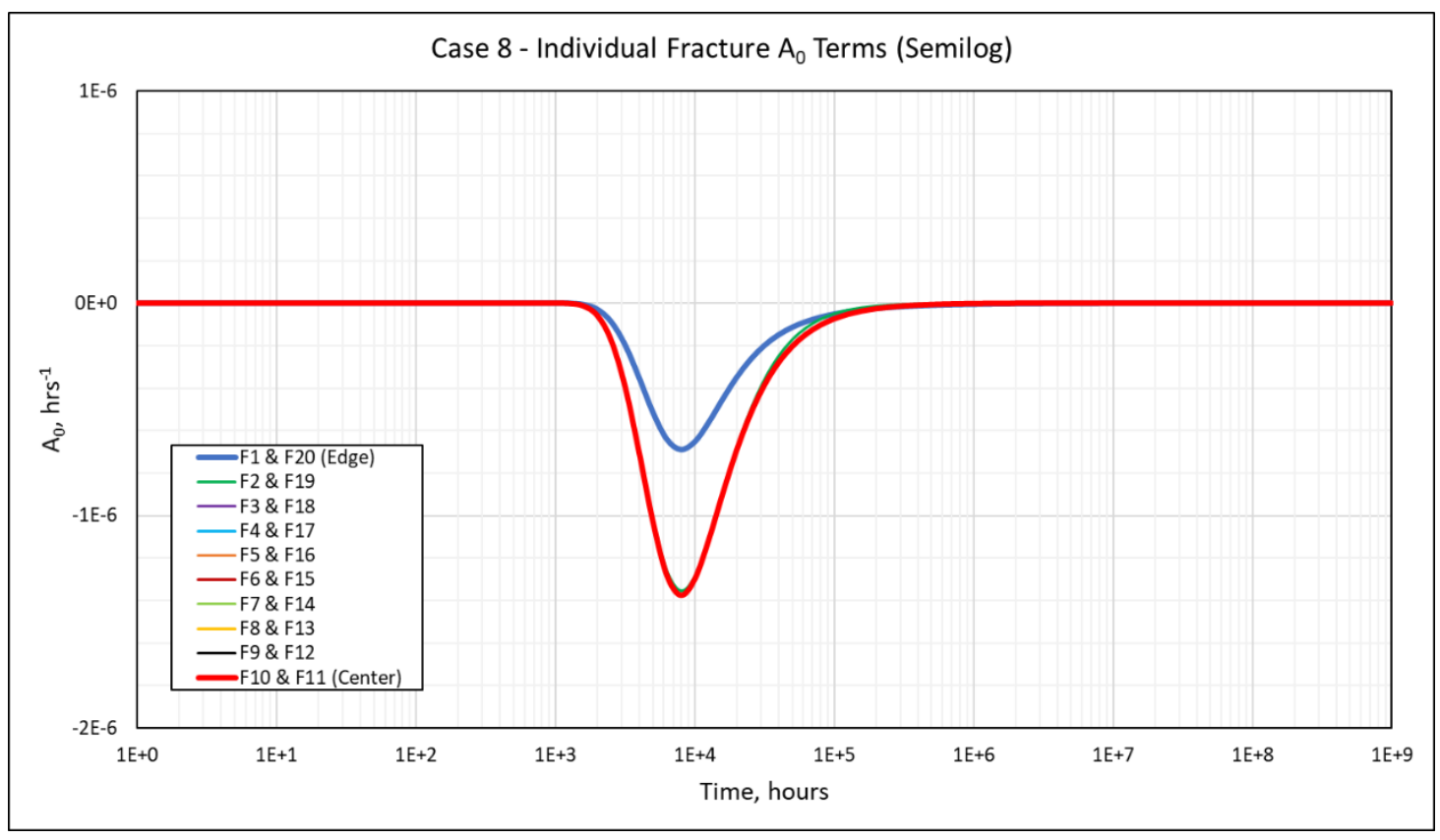

Figure 72 - Case 8 - Individual Fracture Ao Terms 


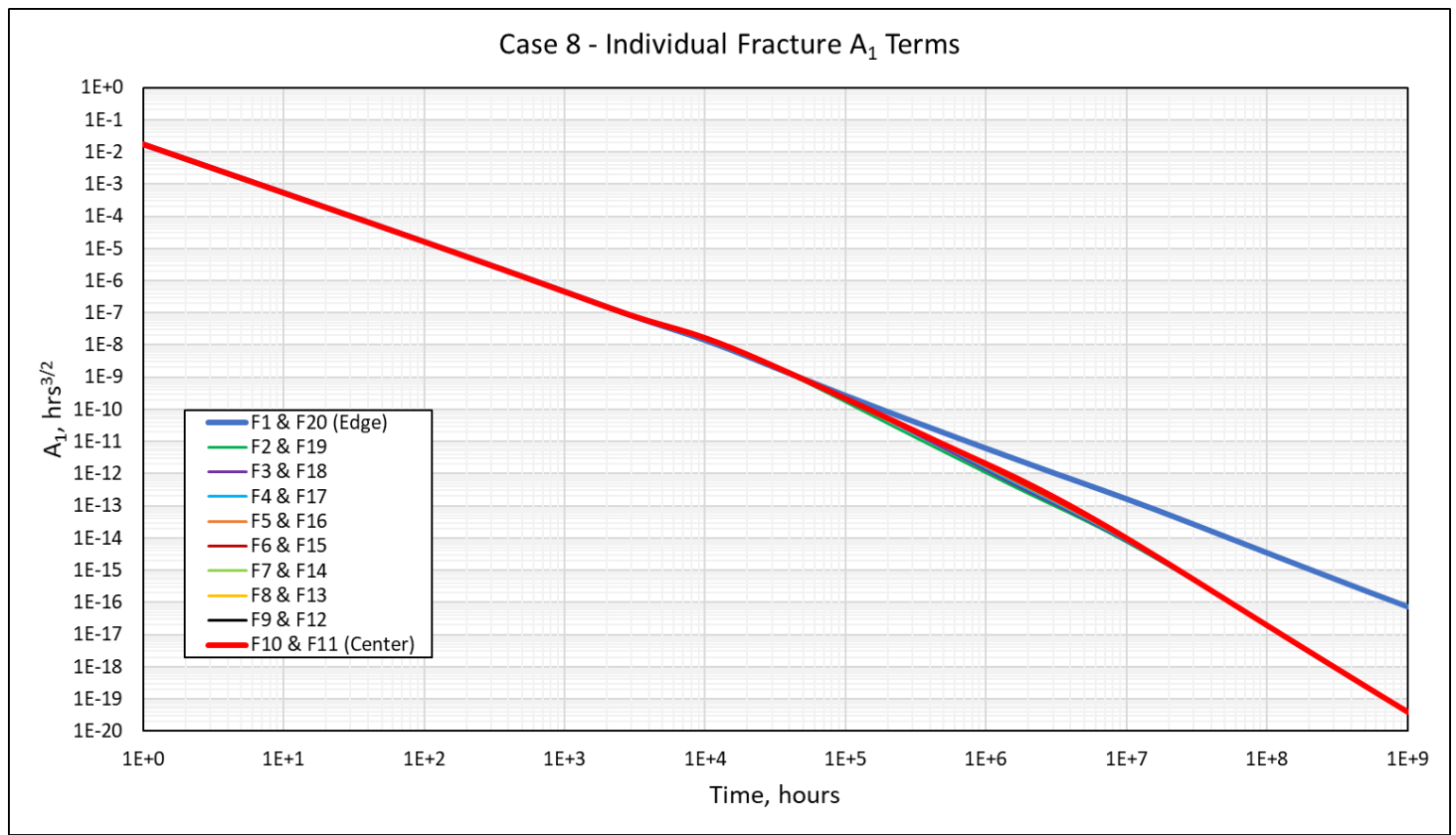

Figure 73 - Case 8 - Individual Fracture A1 Terms 


\section{SRV Flow Mechanisms \& Drainage Volumes}

One additional diagnostic that the asymptotic approximation enables is the calculation of transient drainage volume (Eq. (2.134)). With this we can compute the total reservoir volume accessed by any individual source or sink term in our solution. Applying this to our variable rate solutions, we can further illustrate the mechanisms of fracture interference from the perspective of a single fracture.

$$
\frac{1}{V_{d}\left(t_{e}\right)}=\frac{c_{t}}{t} \frac{d R N P}{d t}
$$

We display this calculation for each fracture in Case 1 in Figure 74. The vertical dotted blue line displays the end of transient linear flow, which will be discussed in more detail in Chapter 3. The horizontal dotted purple line simply shows a rough estimation of the pore volume of the "stimulated reservoir volume" (SRV). This figure illustrates the evolution of drainage areas for individual fractures occurs as fracture interference begins. Recall that Case 1 is a 1-dimensional flow case, and that the reservoir is effectively infinite in the directions in which flow occurs. Up until fracture interference begins, the drainage volume for each fracture grows independently of its neighbors. After the onset of fracture interference, the transient drainage volume comes to a standstill for central fractures but continues to increase for edge fractures. This is the true source of the approximate pseudo steady state (or "pseudo pseudo steady state") signature noted by Song et al. (2011). As time progresses, the central fractures are effectively jailed, and their drainage volumes can only decrease in size. The edge 
fractures however, have an infinite reservoir volume to access, and continue to grow through all time.

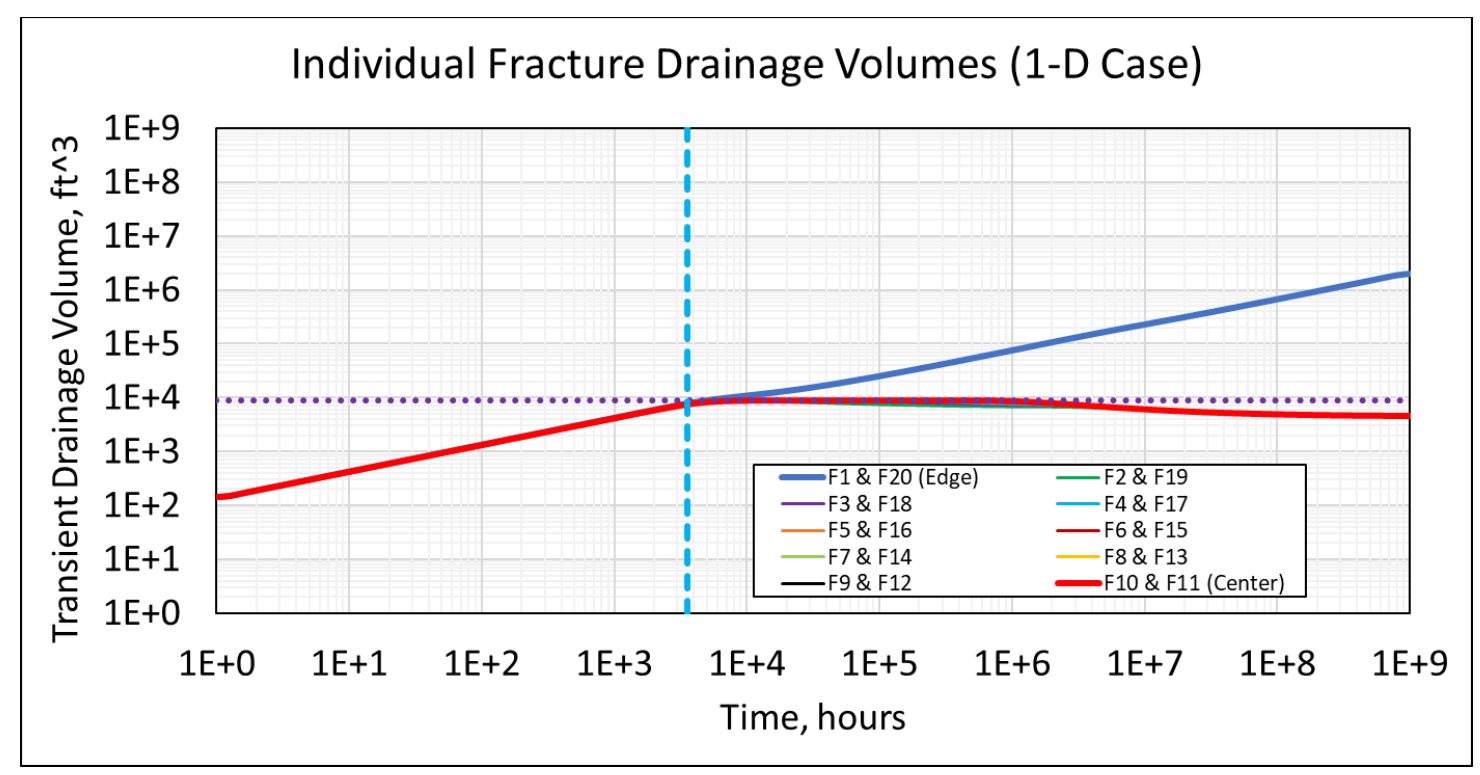

Figure 74 - Individual fracture drainage volumes - Case 1

In Figure 75 we show this plot for Case 3. The dotted blue and purple lines are the same as in Figure 74. Recall that Case 3 uses an infinite 2-dimensional flow assumption. Drainage volume evolution for this case is less constrained given that the available reservoir volume is much greater for a 2-dimensional case than it is for a 1dimensional case. At early time, all fractures act independently of their neighbors and their drainage volumes grow as they access more of the reservoir. At the onset of fracture interference, the drainage volume growth curves diverge. Central fractures don't fully stagnate and decay as they do in the 1-dimensional case. They instead see a short 
stagnation due to an apparent decrease in the available reservoir volume, but then continue to grow after reservoir volume located past the fracture tips is accessed. Edge fractures see no decrease in their drainage volumes growth until slightly after interference when the "pressure signal" from all the other fractures reaches them. Eventually, the drainage volume growth for all fractures stabilizes as collective longterm infinite-acting radial flow begins.

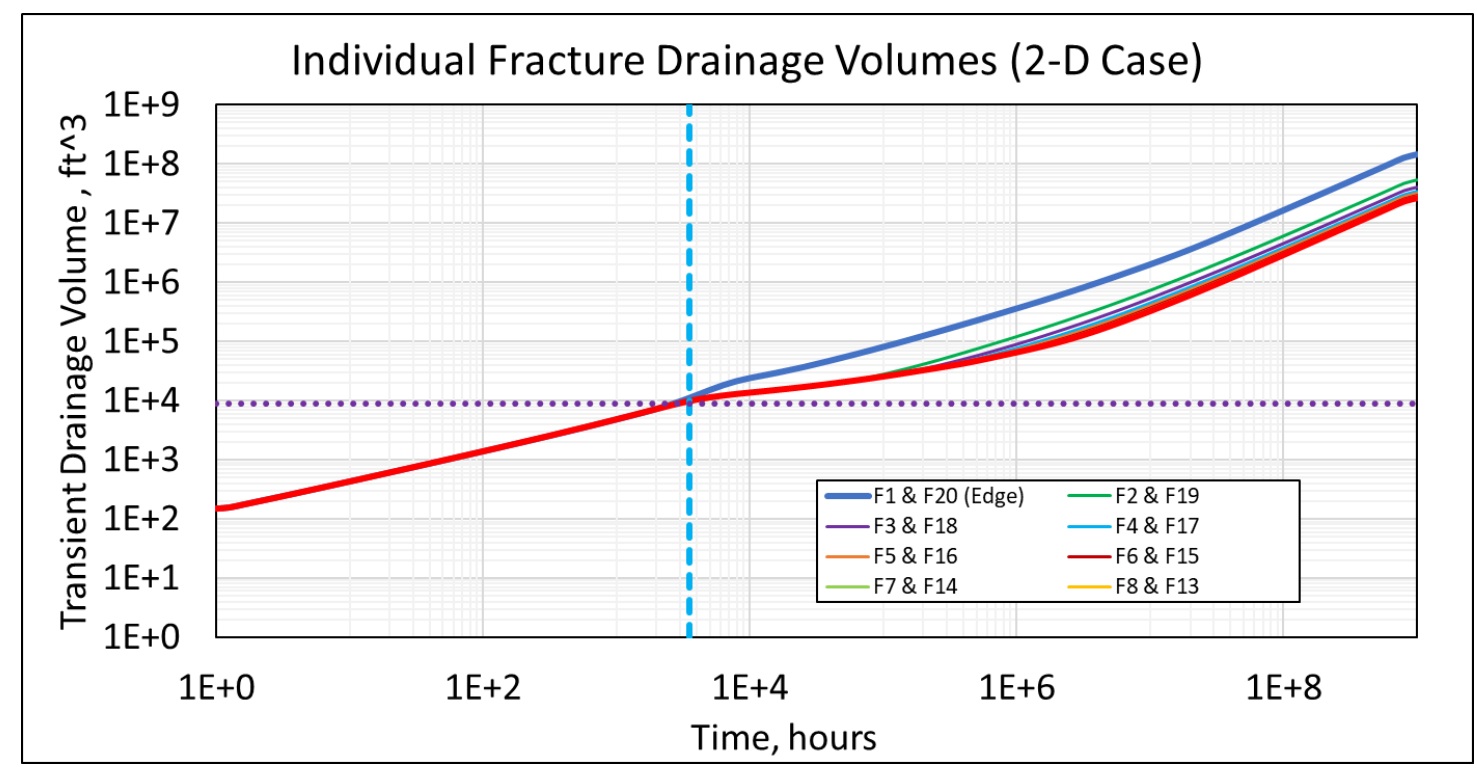

Figure 75 - Individual fracture drainage volumes - Case 3 (after Malone et al. (2019)). Copyright 2019, SPE. Reproduced with permission of SPE. Further reproduction prohibited without permission. 


\section{Conclusions}

\section{Technology Development}

In this chapter we discussed the transient flow behavior of the MTFW geometry, and the diagnostic tools that may be used to interpret production data from MTFW's completed in unconventional shale reservoirs with ultra-low permeability. We discussed the potential applications of work by previous authors in this field. We re-introduced the asymptotic approximation of the diffusivity equation and walked through the derivation of a series of analytic solutions for fluid flow to MTFW's for 1-dimensional and 2dimensional reservoir geometries, as well as constant wellbore rate, constant bottom hole pressure, and variable rate inner boundary conditions. These solutions took advantage of the principle of superposition in space and achieved varying levels of success in testing against reference numerical models.

Our analytic solutions matched reference models very well under constant wellbore rate inner boundary conditions. Both 1-dimensional solutions matched our reference model very well and show promise as potential tools for quick modeling of MTFW's where a 1-dimensional flow assumption might be valid. The 2-dimensional solutions matched our reference model closely, but because the pillbox flow model does not match true transient behavior for all time, it is not a panacea.

Our analytic solutions for constant-BHP flow also performed very well, though not as universally. The 1-term solutions provided an estimate of the reference model at best. Though they were able to accurately capture both early and late time behavior, they struggled to match the reference during middle-time fracture interference. The 2 -term 
solutions provided a significant improvement over the 1-term solutions, taking advantage of the additional degree of freedom offered by a second asymptotic expansion term. Though they failed to perfectly match the non-monotonicity of reference model RNP' curves, they matched early and late time behavior perfectly while very closely approximating diagnostic curves during the period of fracture interference.

\section{Field Mechanisms}

The principle of superposition in space allowed us to match typical MTFW flow behavior on a full-well scale, while also solving for individual fracture flux. With this information and with RTA diagnostics, we showed the mechanisms of fluid flow during SRV drainage and described the proportionality of flow to different fractures during different flow periods, and the variations in influence that different fractures along a horizontal section will see both before and after fracture interference begins.

Most notably, we showed that upon the onset of fracture interference, the drainage volumes seen by hydraulic fractures stagnate due to limited volume availability between the fractures. This results in the apparent pseudo-steady state signature seen on diagnostic plots. We showed definitively that the unit slope signature on diagnostic plots seen during fracture interference occurs not as a result of any real boundary or "SRV" edge, but rather as the result of superimposed pressure drops from fractures reaching their neighbors and causing an apparent pseudo steady state response for a limited period of time. 
Fluid flow in MTFW's will follow a unique progression, depending upon which flow geometry is assumed. For the very simple 1-dimensional case, we can expect production to begin with a linear flow signature, where all fractures act independently of their neighbors, individually exhibiting linear flow. As their drainage areas begin to overlap, fracture interference begins, and we will see a period of approximate pseudo steady state flow occur as central fracture drainage areas become constrained. As time progresses, the flow contribution from central fractures will fall off, and the fullytransient response of the edge fractures will begin to dominate as their drainage volumes are permitted to grow.

For a 2-dimensional case, we see fracture independently exhibit linear flow, resulting in a linear whole-well early response, that transitions to elliptical flow if fractures are spaced far enough apart. Once fracture drainage volumes begin to interact, fracture interference begins, and we see the same approximate pseudo steady state signature occur that is seen in the 1-dimensional case. However, the central fractures will see a short stagnation, followed by renewed drainage volume growth due to available reservoir volume beyond the fracture tips. Eventually, we see the fractures transition at very late time to a collective radial flow signature as their drainage volume growth stabilizes. 


\section{CHAPTER III}

\section{INVESTIGATION OF THE END OF LINEAR FLOW*}

\section{Background and Introduction}

In Chapter 2 we briefly introduced the "end of linear flow" concept and pointed out that some ambiguity exists in its definition and usage. In this chapter, we will review the physical mechanisms of the end of linear flow and discuss its ramifications on MTFW production analysis. We will then review definitions and equations for the end of linear flow that prior authors provided and discuss the ambiguity that we cite. Finally, we will provide an alternative definition of the end of linear flow - one based on the principles of the asymptotic approximation of the diffusivity equation. We will demonstrate the advantages of this definition, the foremost being independence from inner boundary conditions and flow geometry. We will provide a field example from the Montney Shale that demonstrates our formulation's accuracy and discuss the ramifications of our proposed definition of the end of linear flow.

\section{MTFW Flow Example}

The MTFW geometry is complex, and a varying set of flow behaviors may present themselves during production from MTFW's. Which flow regimes appear will depend upon reservoir parameters such as permeability, fluid viscosity, and porosity, as

\footnotetext{
* Reprinted with permission from "Characterization of Multiple Transverse Fractured Wells Using the Asymptotic Approximation of the Diffusivity Equation" by Andrew Malone, Michael J. King, and Zhenzhen Wang, 2019. SPE Europec featured at the $81^{\text {st }}$ EAGE Conference and Exhibition, Copyright [2019] by the Society of Petroleum Engineers
} 
well as completion parameters such as fracture spacing, fracture half-length, fracture conductivity, and fracture complexity. Here we present a generalized model to illustrate the progression of flow regimes encountered during production from an MTFW completed in an ultra-tight formation.

Our example model consists of a bounded rectangular reservoir with a 10fracture MTFW placed in its center (Figure 76). This model includes a "stimulated reservoir volume" (SRV), or a rectangular zone of enhanced permeability that represents reservoir rock whose permeability increased as a result of hydraulic fracturing. Though the same progression of flow regimes occurs regardless of whether an SRV exists, the presence of an SRV extends the duration of the approximate pseudo steady state, or fracture interference, flow regime, enabling stronger visualization. Table 3 lists the model properties. For clarity, we define the dimensionless fracture conductivity (“Dimensionless Frac. Cond." in Table 3) by the same definition as Lee et al. (2003) (Equation (3.1)). Note that since the fractures sit within the SRV region, the " $k$ " parameter in (3.1) will refer to the SRV permeability. Because the contrast between the SRV permeability and fracture permeability is low, the fractures in this model have finite conductivity, allowing us to visualize early bilinear flow as well as early linear flow.

$$
C_{r D}=\frac{w_{f} k_{f}}{\pi k\left(2 x_{f}\right)}
$$




\begin{tabular}{|rcl|}
\hline Example Model Properties \\
\hline Rock \& Fluid Properties & \\
\hline Permeability, $\mathrm{k}=$ & $\mathbf{0 . 0 0 0 0 2}$ & $\mathrm{md}$ \\
Porosity, $\phi=$ & $\mathbf{6 \%}$ & \\
Total Compressibility, $\mathrm{c}_{\mathrm{t}}=$ & $\mathbf{2 * 1 0 ^ { - 5 }}$ & $\mathrm{psi}^{-1}$ \\
Oil Viscosity, $\mu=$ & $\mathbf{1}$ & $\mathrm{cp}$ \\
Pay Height, $\mathrm{h}=$ & $\mathbf{2 5}$ & $\mathrm{ft}$ \\
Pormation Volume Factor, $\mathrm{B}=$ & $\mathbf{1}$ & $\mathrm{rb} / \mathrm{stb}$ \\
Diffusivity, $\alpha=$ & $\mathbf{0 . 0 4 4}$ & $\mathrm{ft} / \mathrm{hr}$ \\
SRV Permeability, $\mathrm{k}_{\mathrm{SRV}}=$ & $\mathbf{0 . 0 0 0 2}$ & $\mathrm{md}$ \\
& & \\
Model Fracture & Properties & \\
\hline Fracture Half-Length, $\mathrm{x}_{\mathrm{f}}=$ & $\mathbf{5 2 . 5}$ & $\mathrm{ft}$ \\
Fracture Spacing, $\mathrm{x}_{\mathrm{s}}=$ & $\mathbf{6 4 . 2}$ & $\mathrm{ft}$ \\
\hline Number of Fractures, $\mathrm{N}_{\mathrm{f}}=$ & $\mathbf{1 0}$ & $\mathrm{fractures}$ \\
Fracture Permeability, $\mathrm{k}_{\mathrm{f}}=$ & $\mathbf{1 0 0}$ & $\mathrm{md}$ \\
Fracture Width, $\mathrm{w}_{\mathrm{f}}=$ & $\mathbf{0 . 0 1}$ & $\mathrm{ft}$ \\
Dimensionless Frac. Cond., $\mathrm{C}_{\mathrm{rd}}=$ & $\mathbf{1 5 . 2}$ & Finite Cond. \\
\hline
\end{tabular}

Table 3 - Example Model Properties

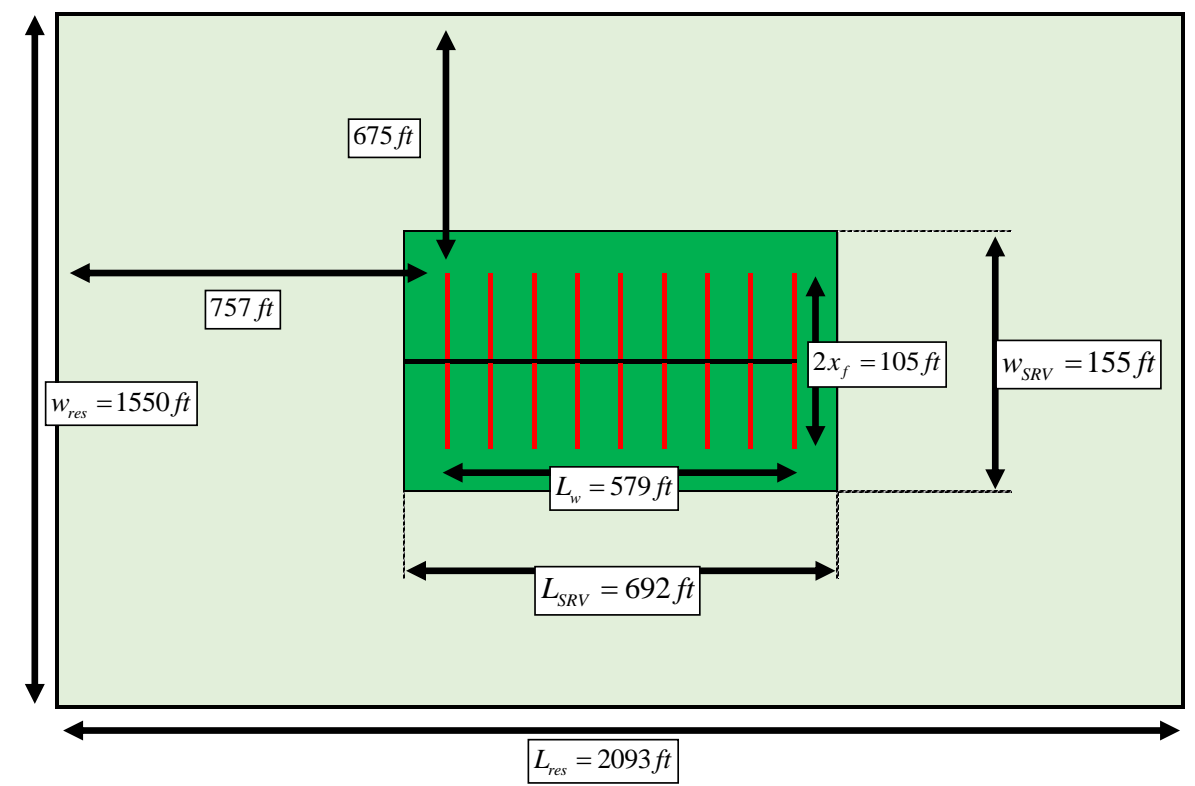

Figure 76 - Example Model Diagram (from Malone et al. (2019)). Copyright 2019, SPE. Reproduced with permission of SPE. Further reproduction prohibited without permission. 
Applying a constant-BHP inner boundary condition to maintain a pressure drawdown of $2500 \mathrm{psi}$, we ran a flow simulation to the maximum allowable time in the commercial simulator, Eclipse. In Figure 77 we plot the rate-normalized pressure (RNP) and its derivative with respect to the natural logarithm of material balance time (RNP') versus material balance time $\left(t_{m b}\right)$. Plotting RNP and RNP' versus material balance time allows us to plot constant-BHP data as an equivalent constant-rate diagnostic plot, which is much easier to interpret. Figure 78 includes the same diagnostic plot, but with labels for each flow regime encountered. Figure 79 through Figure 84 include drainage area maps of the example model. In these maps, pink-shaded cells are those cells whose pressure has dropped below initial reservoir pressure, and blue cells are those cells whose pressure still equals their initial pressure. In short, these maps show the drainage volume of the well, and of each individual fracture, as pink areas.

$$
\begin{gathered}
t_{m b}=\frac{Q}{q} \\
R N P=\frac{\Delta p_{w f}}{q} \\
R N P^{\prime}=\frac{d R N P}{d \ln \left(t_{m b}\right)}
\end{gathered}
$$

\section{Early Time - Bilinear Flow}

Flow to a hydraulic fracture will begin with either linear or bilinear flow, depending upon the fracture conductivity (Lee et al. 2003). The model presented here 
includes finite conductivity fractures and first exhibits bilinear flow, indicated by a quarter slope on the RNP' curve of Figure 77. During bilinear flow, the well drains both the volume of the fractures and the volume of the surrounding matrix rock (Figure 79).

\section{Early/Middle Time - Linear Flow}

Eventually, the fractures become fully drained and bilinear flow ends. The fractures begin to act as plane sources and fluid flow towards the fractures, perpendicular to their planar orientation, dominates the wellbore diagnostic response (Figure 80). This shows up as a half-slope on the RNP' curve on our diagnostic plot in Figure 77 and Figure 78.

\section{Middle Time - Fracture Interference (Approximate Pseudo Steady State)}

As the fractures continue to drain the surrounding rock matrix, their drainage volumes eventually overlap (Figure 81). Fractures draw down upon each other and available undrained rock volume decreases, so we see an acceleration occur on the RNP curve (Figure 78). Stagnation lines form at the boundary between adjacent fractures' drainage areas. Much in the same way that an image well can represent a nearby boundary, each fracture's neighbors create virtual boundaries between them. This elicits an apparent pseudo steady state response - a unit slope - on the RNP' curve.

The onset of this approximately pseudo steady state (APSS) signature coincides with the time at which fractures begin to interact and is the point in time that we refer to as the "end of linear flow", or $t_{\text {elf. }}$ Accurate detection of this time can yield an estimate of 
the permeability of the rock between hydraulic fractures, and will be discussed in more detail in Section 3.4.

\section{Late Time - Pseudolinear Flow}

Once the fractures drain the volume between them, they begin to act as a single unit and adopt a collective flow pattern. This begins as what Song et al. (2011) coined "pseudolinear flow", where each fracture drains its own channel-shaped volume, parallel to its orientation plane. At the whole-well resolution, this appears as a single shared rectangular drainage area (Figure 82). This appears as a brief half-slope on the RNP' curve (Figure 78)

\section{Late Time - Radial Transition}

Next, we see the collective drainage volume continue to propagate outwards, forming an elliptical shape that transitions to a circular shape (Figure 83). This transition flattens the RNP' curve as available reservoir volume increases again.

\section{Late Time - Pseudo Steady State (Boundary-Dominated) Flow}

Finally, the well's drainage volume reaches the reservoir's outer boundary (Figure 84). The boundary could be an even less permeable rock but would more likely represent a stagnation line between adjacent wells. The well will continue to drain from more of the reservoir volume until we see flow from the entire reservoir and enter a true pseudo steady state (or boundary-dominated) flow period (Figure 85). 


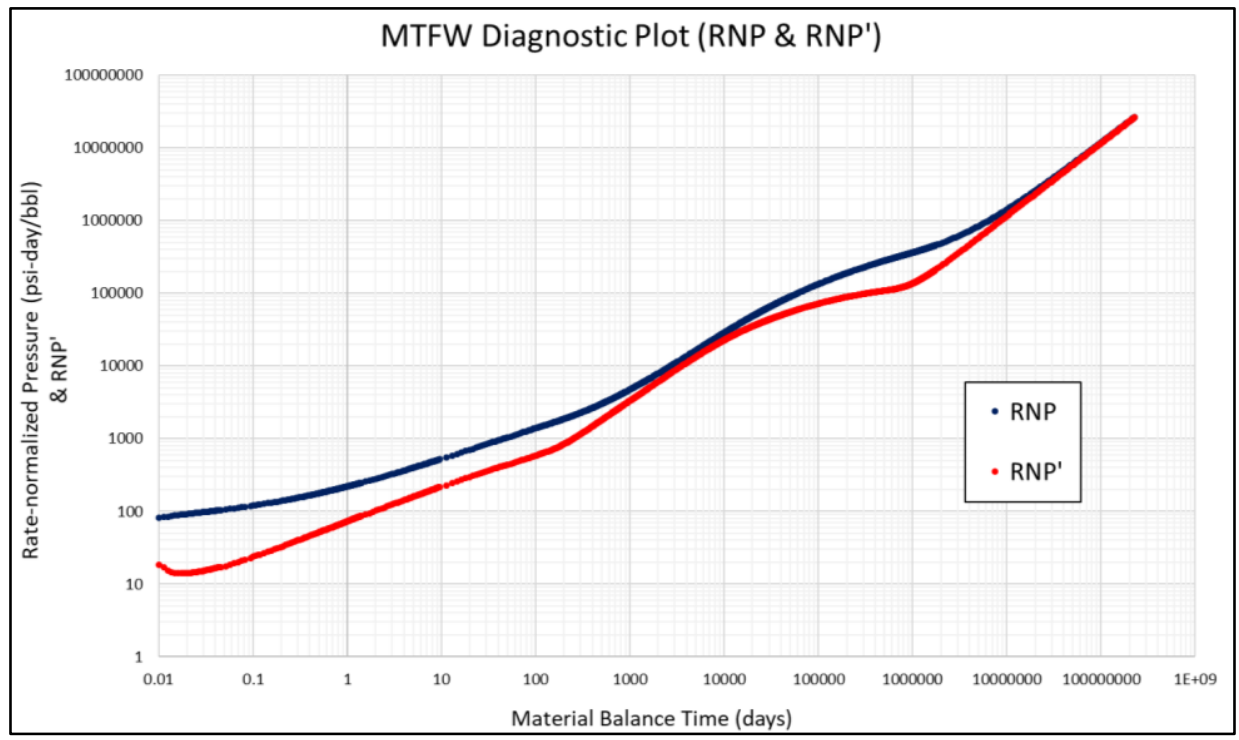

Figure 77 - Example MTFW Model Diagnostic Plot (after Malone et al. (2019)) Copyright 2019, SPE. Reproduced with permission of SPE. Further reproduction prohibited without permission.

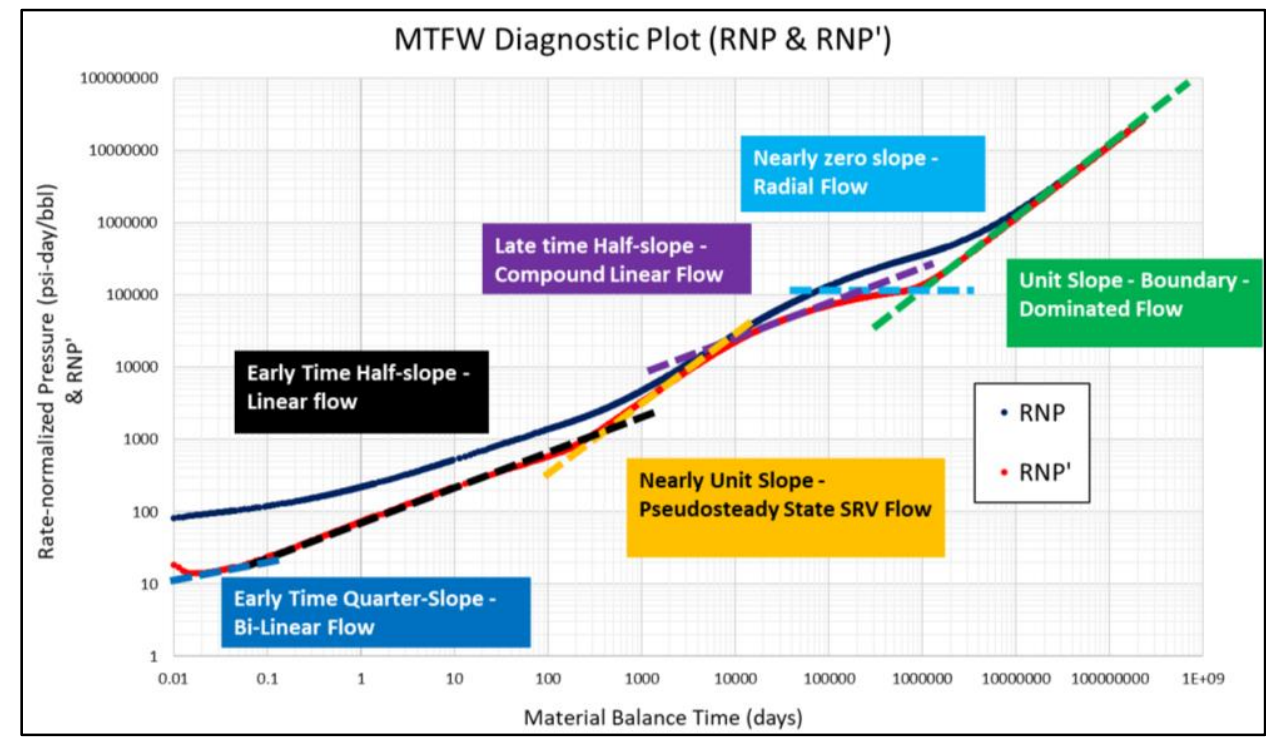

Figure 78 - Example MTFW Model Diagnostic Plot (Flow Regimes Labelled) (after Malone et al. (2019)). Copyright 2019, SPE. Reproduced with permission of SPE. Further reproduction prohibited without permission. 


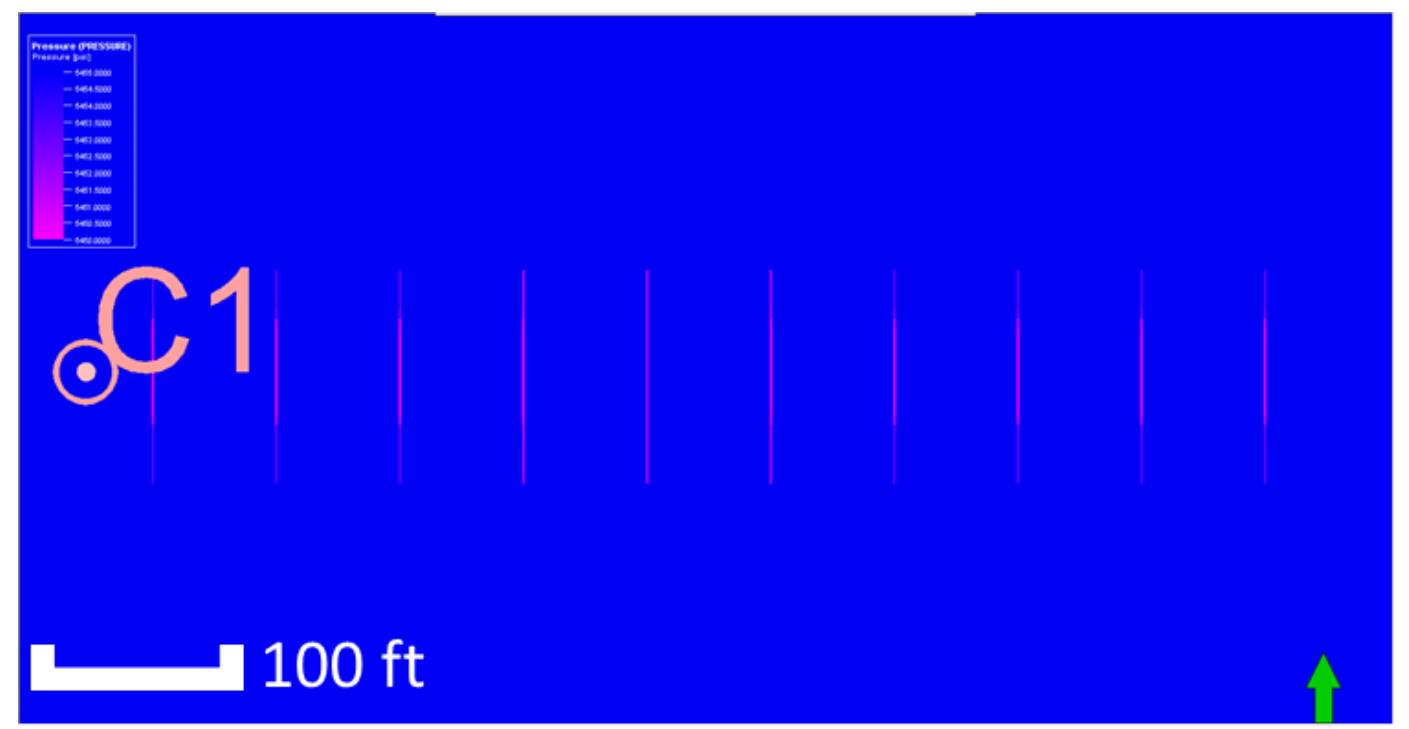

Figure 79 - Early Time - Bilinear Flow (from Malone et al. (2019)). Copyright 2019, SPE. Reproduced with permission of SPE. Further reproduction prohibited without permission.

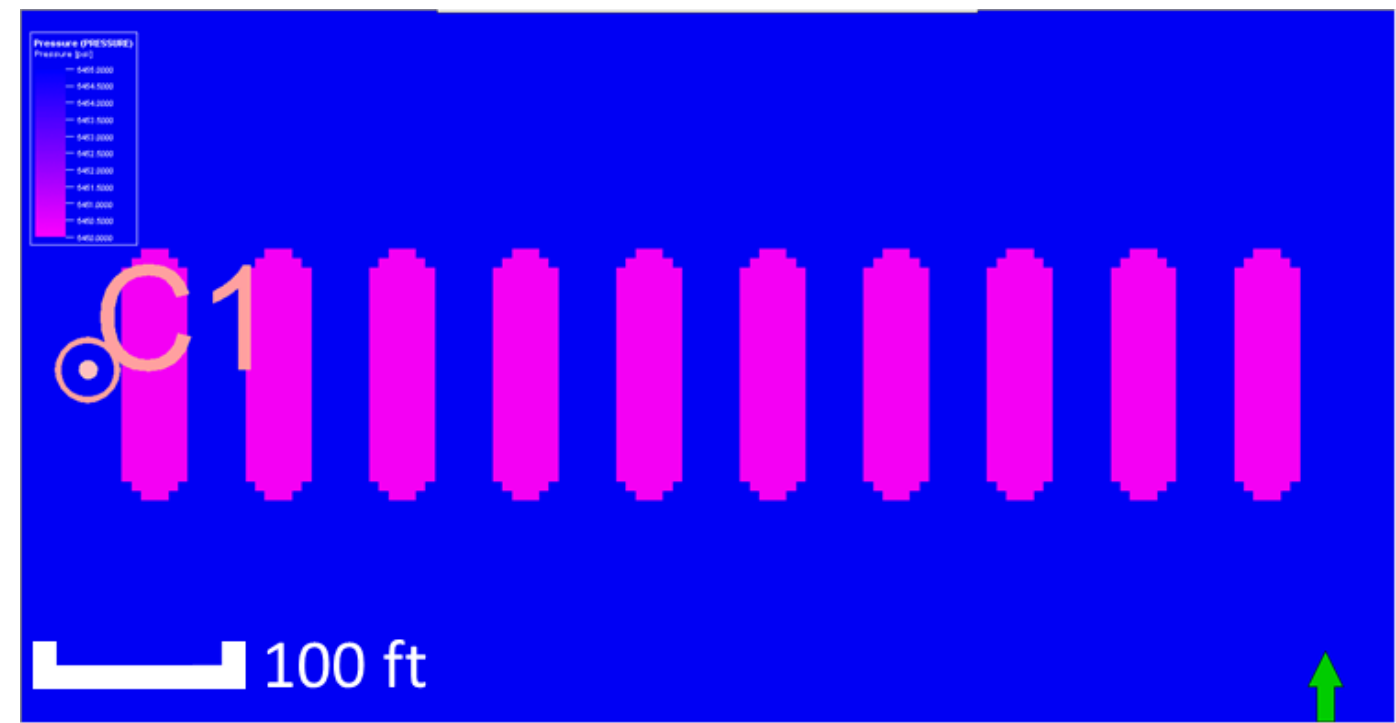

Figure 80 - Early/Middle Time - Linear Flow (from Malone et al. (2019)). Copyright 2019, SPE. Reproduced with permission of SPE. Further reproduction prohibited without permission. 


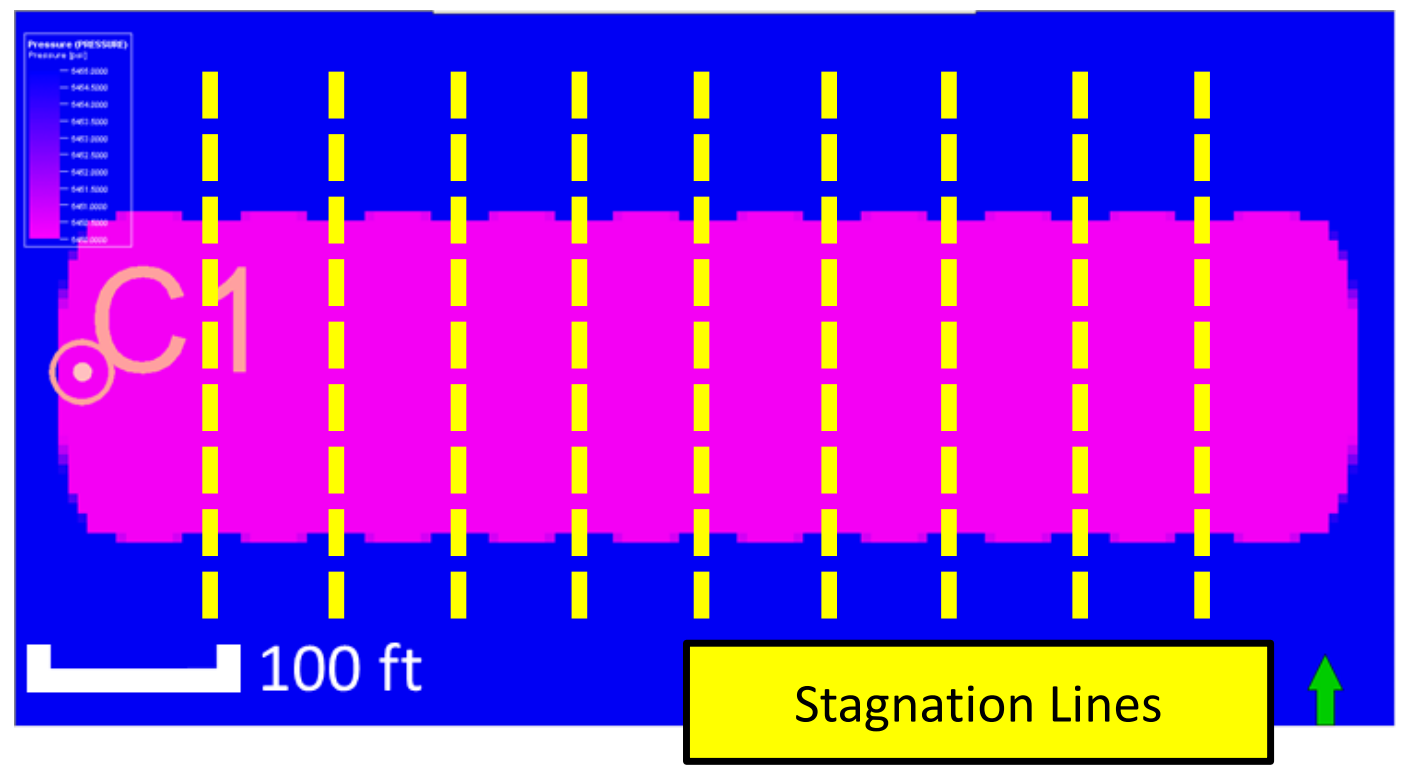

Figure 81 - Middle Time - Fracture Interference \& Apparent Pseudo Steady State (from Malone et al. (2019)). Copyright 2019, SPE. Reproduced with permission of SPE. Further reproduction prohibited without permission.

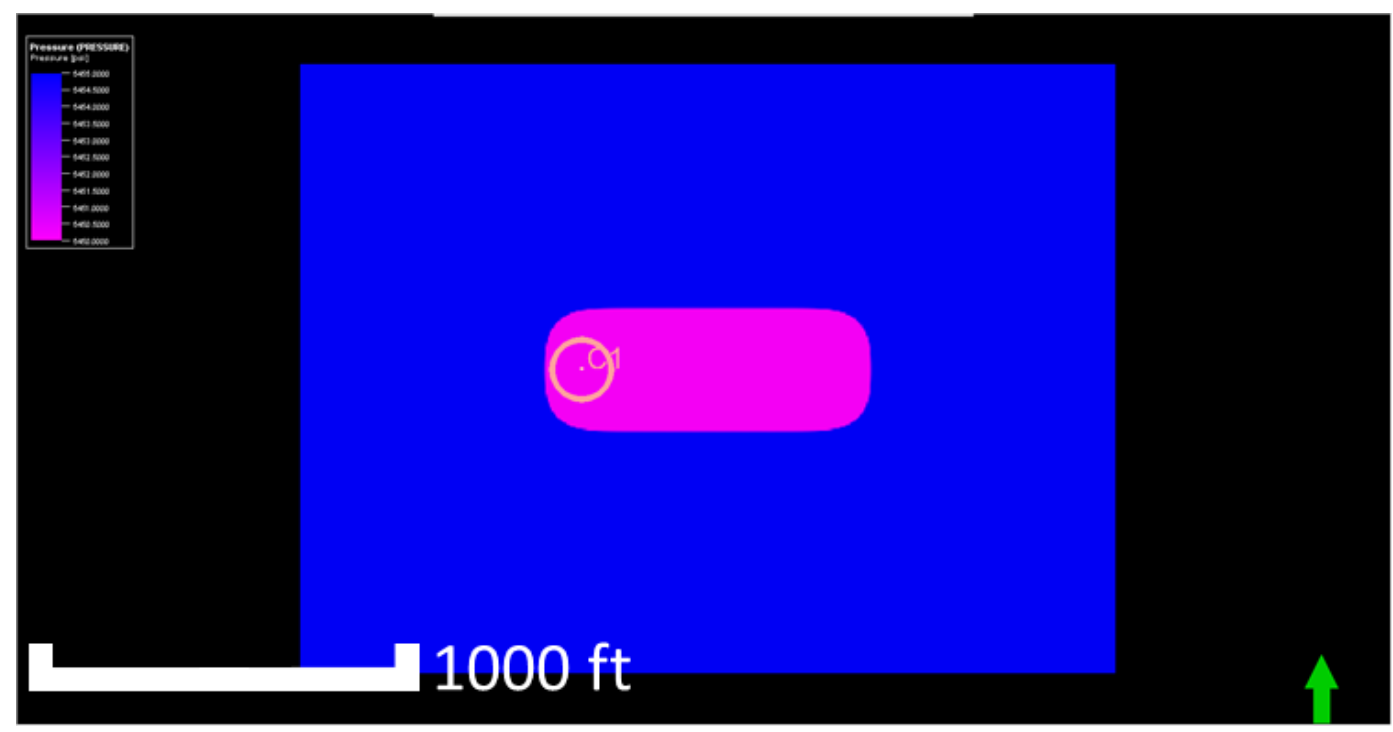

Figure 82 - Late Time - Compound Linear Flow 


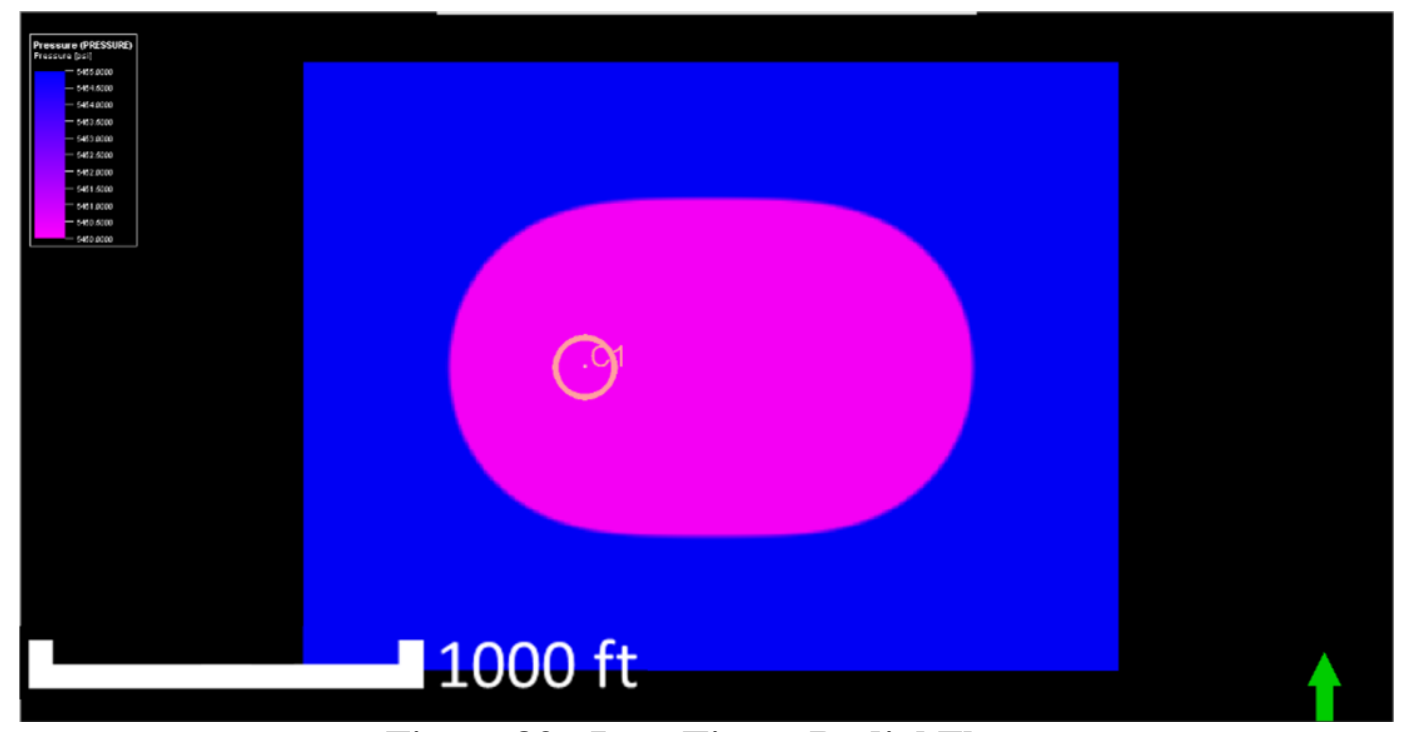

Figure 83 - Late Time - Radial Flow

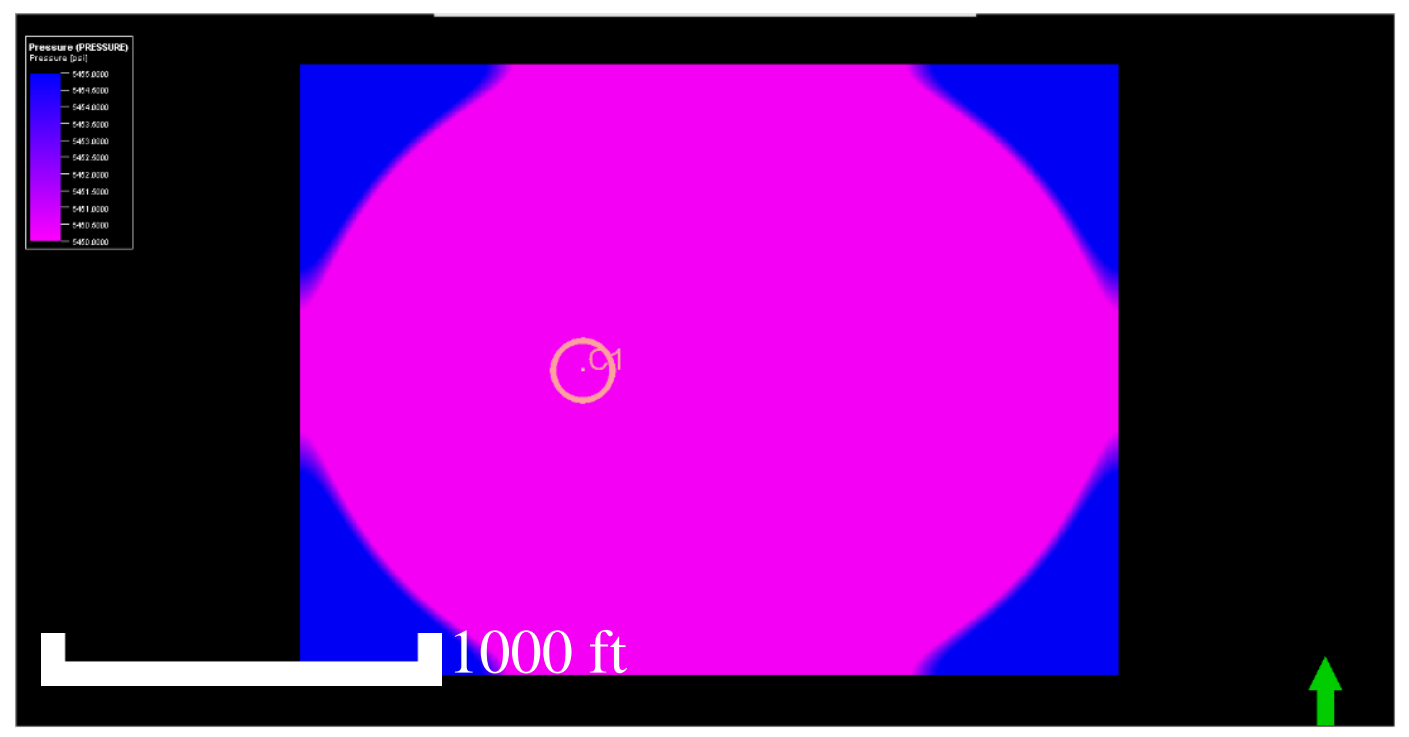

Figure 84 - Late Time - Transition to Pseudo Steady State 


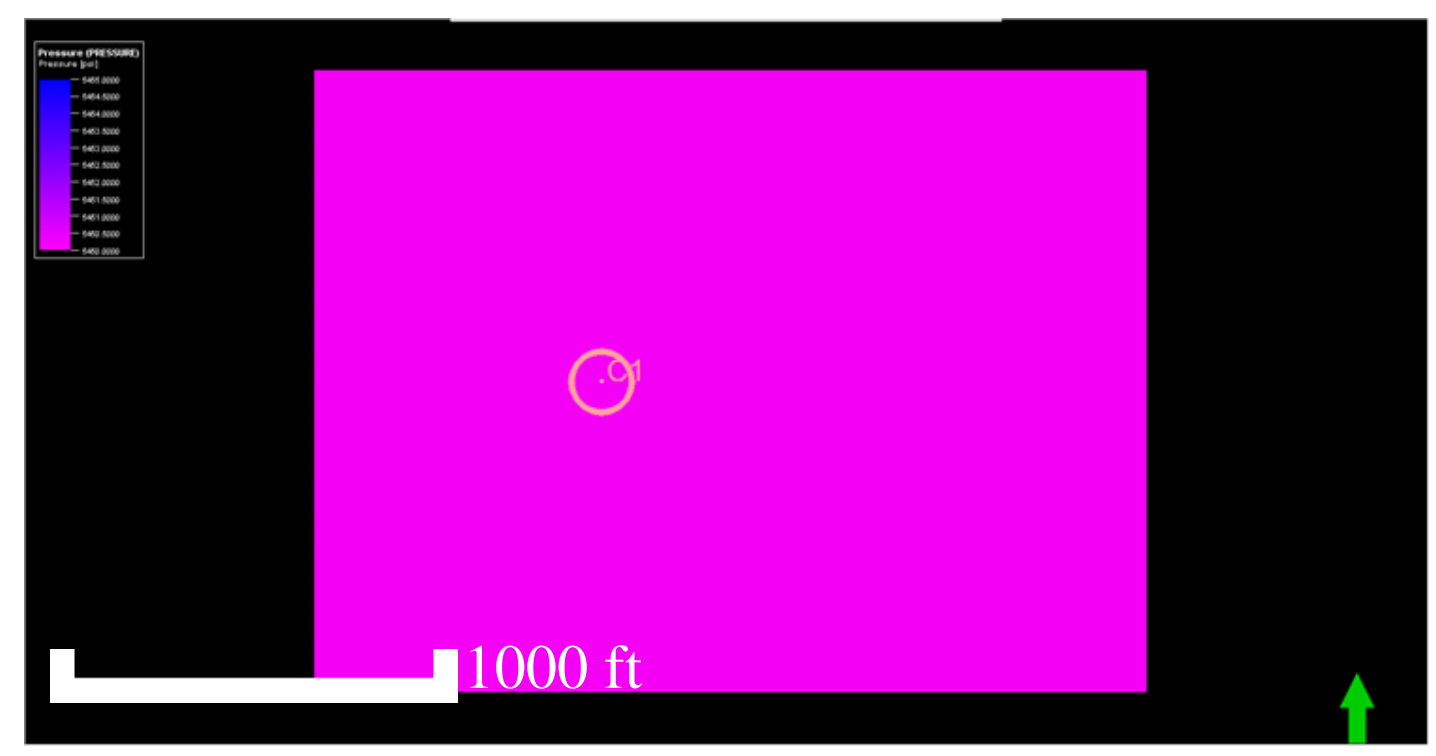

Figure 85 - Late Time - True Pseudo Steady State

\section{Previous Definitions of the End of Linear Flow}

Recall the transition between linear flow and fracture interference (APSS flow).

As we demonstrated in Chapter 2, this APSS signature comes from the superposition of a series of pressure drawdowns induced on each fracture by its neighbors. In other words, approximate pseudo steady state flow begins when fractures' pressure pulses become detectable by their neighbors. It stands to reason that if we can detect the onset of this flow regime, we can use pressure propagation relationships to quantify reservoir properties (most importantly permeability). A useful $t_{e l f}$ formulation should tell us when interference begins, but in a way that is detectable and relates to fluid flow fundamentals (Figure 86).

The field of interference testing already uses this concept (Lee et al. 2003), and many authors discuss it within the context of linear flow. Various authors propose 
different definitions of the end of linear flow (Lee and Spivey 2013; Song et al. 2011; Song and Ehlig-Economides 2011; Wattenbarger et al. 1998). Upon review it becomes clear that a great ambiguity exists in this space. For illustration of this ambiguity, see Figure 87 where we plot each of the cited $t_{\text {elf }}$ equations against an MTFW diagnostic plot.

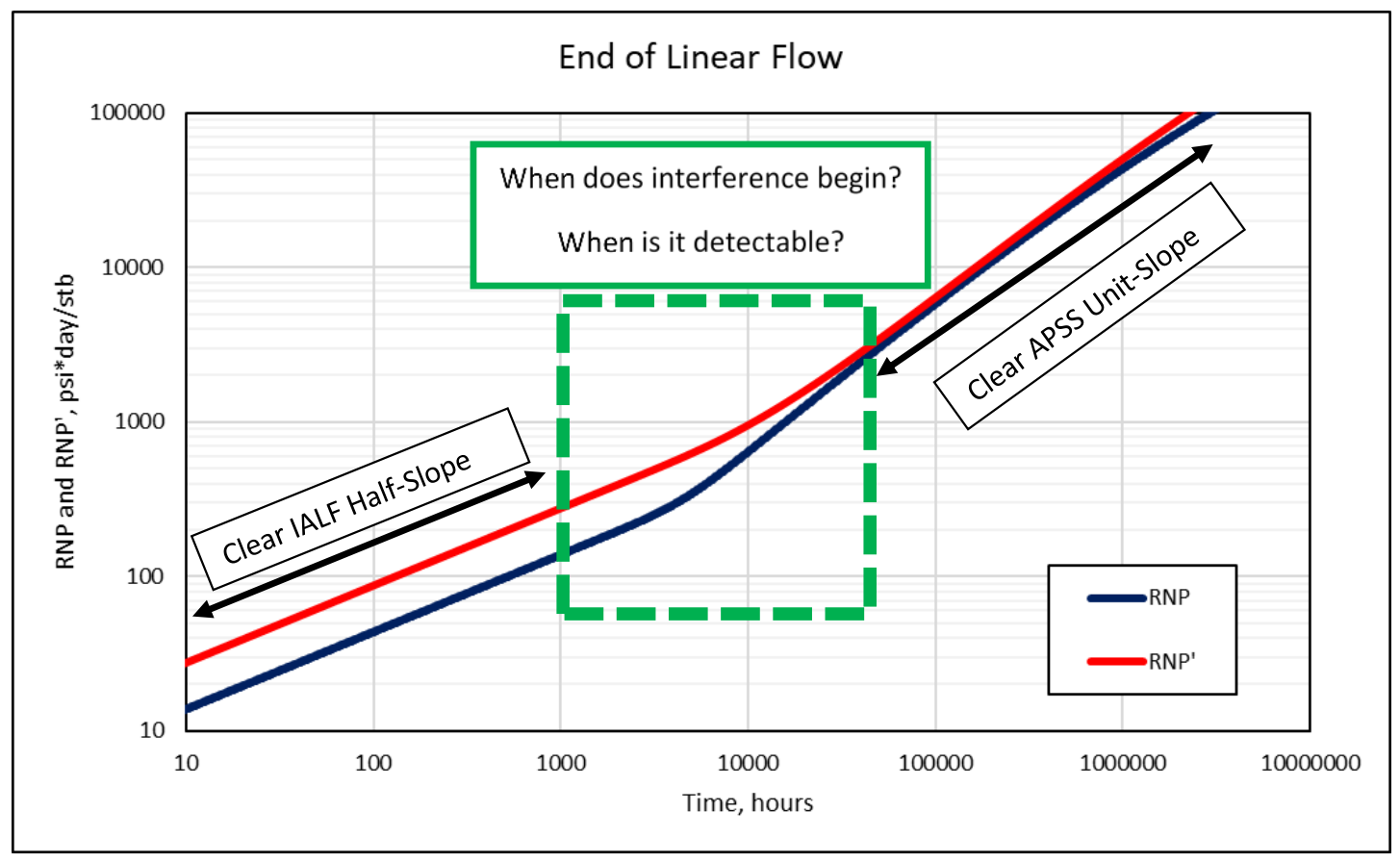

Figure 86 - End of Linear Flow 


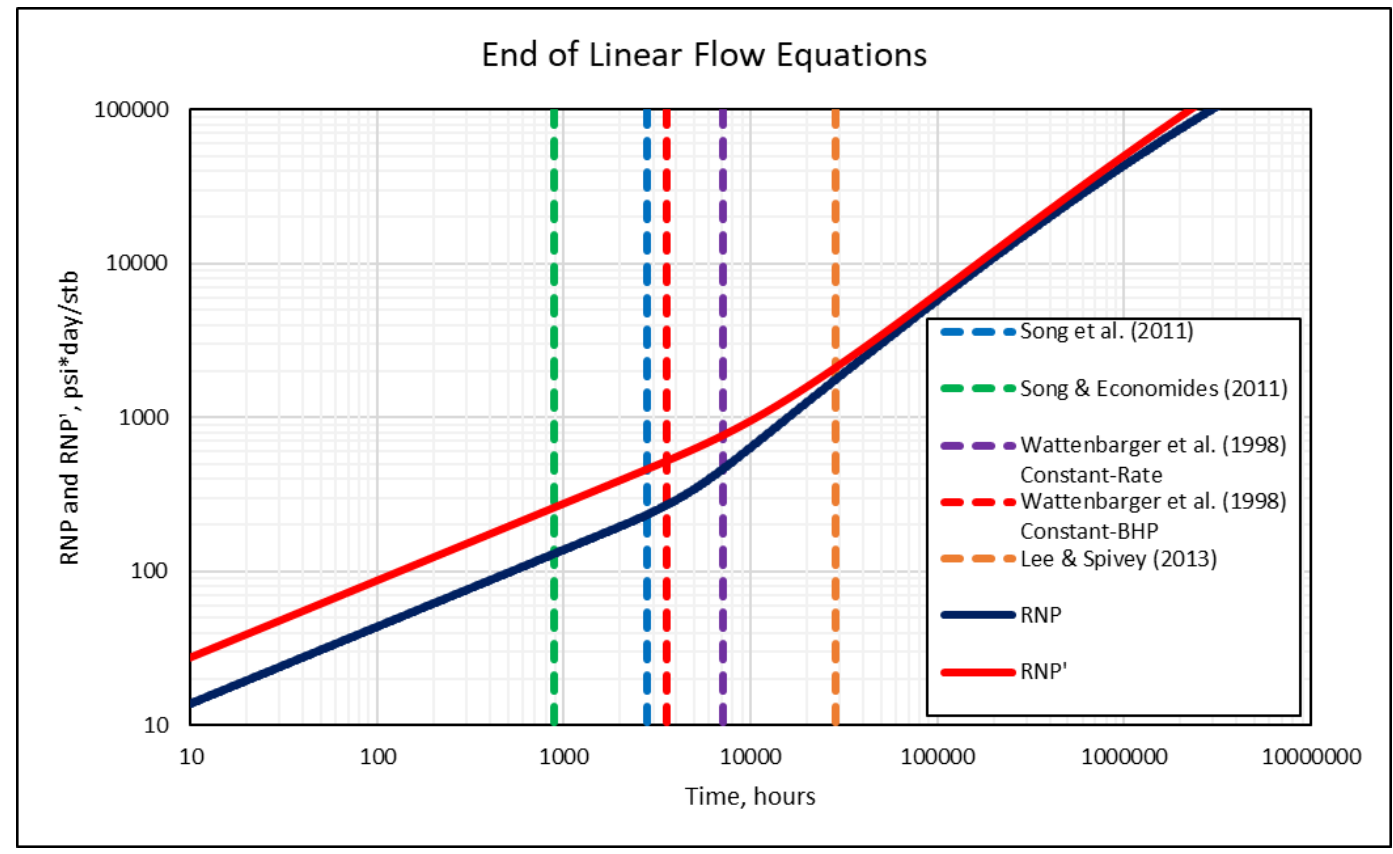

Figure 87 - End of Linear Flow Equations (from Malone et al. (2019)). Copyright 2019, SPE. Reproduced with permission of SPE. Further reproduction prohibited without permission.

End of Linear Flow - Song et al. (2011)

Song et al. (2011) defines the end of linear flow by an equation provided in Ehlig-Economides (1992) (Equation (3.5)). This equation was originally developed as a depth of investigation equation for linear flow to a vertical well in a channel - not flow to a fracture (Ehlig-Economides 1992). Song et al. (2011) states that the end of linear flow occurs when the depth of investigation from equally-spaced fractures equals half the fracture spacing, or when it reaches the stagnation point between fractures. For later comparison with other equations, we show this equation in a reduced form in Eq. (3.6). 


$$
\begin{gathered}
t_{\text {elf }}=\frac{948 \pi \phi \mu c_{t}\left(\frac{x_{s}}{2}\right)^{2}}{4 k} \\
t_{\text {elf }}=\frac{\pi x_{s}^{2}}{64 \alpha}
\end{gathered}
$$

\section{End of Linear Flow - Song and Ehlig-Economides (2011)}

Song and Ehlig-Economides (2011) defines the end of linear flow by another equation provided in Ehlig-Economides (1992) (Equation (3.7)). Interestingly, this equation is another depth of investigation relationship. It was intended for what EhligEconomides (1992) calls "pseudo-linear flow", which they define as linear flow to a fracture. Song and Ehlig-Economides (2011) states that the end of linear flow occurs when the depth of investigation from equally-spaced fractures equals half the fracture spacing, or when it reaches the stagnation point between fractures. We show this equation in simplified form in Eq. (3.8).

$$
\begin{gathered}
t_{e l f}=\frac{948 \phi \mu c_{t} x_{s}^{2}}{16 k} \\
t_{\text {elf }}=\frac{x_{s}^{2}}{64 \alpha}
\end{gathered}
$$

The only real difference between (3.7) and (3.5) is (3.7)'s omission of the factor of $\pi$ seen in (3.5). 


\section{End of Linear Flow - Wattenbarger et al. (1998)}

Wattenbarger et al. (1998) introduced the well-known Wattenbarger Type Curve, which describes the behavior of a single planar source draining a rectangular region (Figure 88). They developed a type curve for both constant well rate (Equation (3.9)) and constant bottom hole pressure (Equation (3.10)) inner boundary conditions (IBC's).

$$
\begin{gathered}
\left(\frac{x_{f}}{y_{e}}\right) p_{w D}=\frac{\pi}{2}\left\{\frac{1}{3}+t_{D y_{e}}-\frac{2}{\pi^{2}} \sum_{n=1}^{\infty} \frac{1}{n^{2}} \exp \left(-n^{2} \pi^{2} t_{D y_{e}}\right)\right\} \\
\left(\frac{x_{f}}{y_{e}}\right) \frac{1}{q_{D}}=\frac{\pi}{4 \sum_{n_{\text {odd }}}^{\infty} \exp \left(-\frac{n^{2} \pi^{2}}{4} t_{D y_{e}}\right)}
\end{gathered}
$$

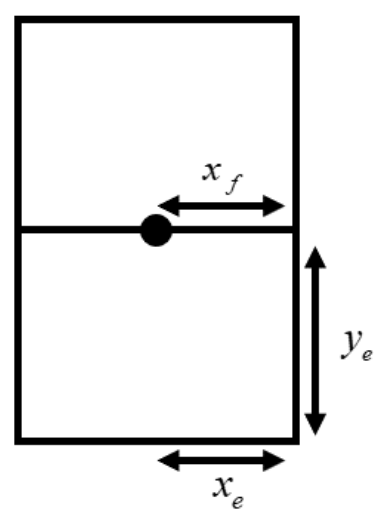

Figure 88 - Wattenbarger Type Curve Geometry (Modeled after Wattenbarger et al. (1998)) 
The Wattenbarger type curve enables users to calculate the ratio $\frac{x_{f}}{y_{e}}$ by fitting field data to the type curve presented in Figure 89. Wattenbarger et al. (1998) identified the beginning of boundary-dominated behavior on both curves in Figure 89 by visually picking the point in dimensionless time where each curve lifted from a half-slope. They name this time the "time, end of half slope" or "tehs", and this value is analogous to our $t_{e l f .}$ They identified a different value for each inner boundary condition (IBC) in dimensionless time. For a constant-BHP IBC, they list Eq. (3.11), and for a constant-rate IBC, they list Eq. (3.12).

$$
\begin{gathered}
t_{\text {ehs }}=0.25 \\
t_{\text {ehs }}=0.5
\end{gathered}
$$

Using the definition of dimensionless time listed in Wattenbarger et al. (1998) (Equation (3.13)), and considering that $y_{e}$ equals half $x_{s}$ (Eq. (3.14)), we can obtain an equivalent $t_{\text {elf }}$ value in real time for each IBC (constant-rate in Eq. (3.15) and constant-BHP in Eq. (3.16)).

$$
\begin{gathered}
t_{y_{e} D}=\frac{0.0002637 k t}{\phi \mu c_{t} y_{e}^{2}} \\
y_{e}=\frac{x_{s}}{2} \\
t_{\text {elf }}=\frac{948 \phi \mu c_{t} x_{s}^{2}}{2 k}
\end{gathered}
$$




$$
t_{\text {elf }}=\frac{948 \phi \mu c_{t} x_{s}^{2}}{4 k}
$$

Listed in simplified terms, we have Eq. (3.17) and Eq. (3.18) for constant-rate and constant-BHP IBC's, respectively.

$$
\begin{gathered}
t_{\text {elf }}=\frac{x_{s}^{2}}{8 \alpha} \\
t_{\text {elf }}=\frac{x_{s}^{2}}{16 \alpha}
\end{gathered}
$$

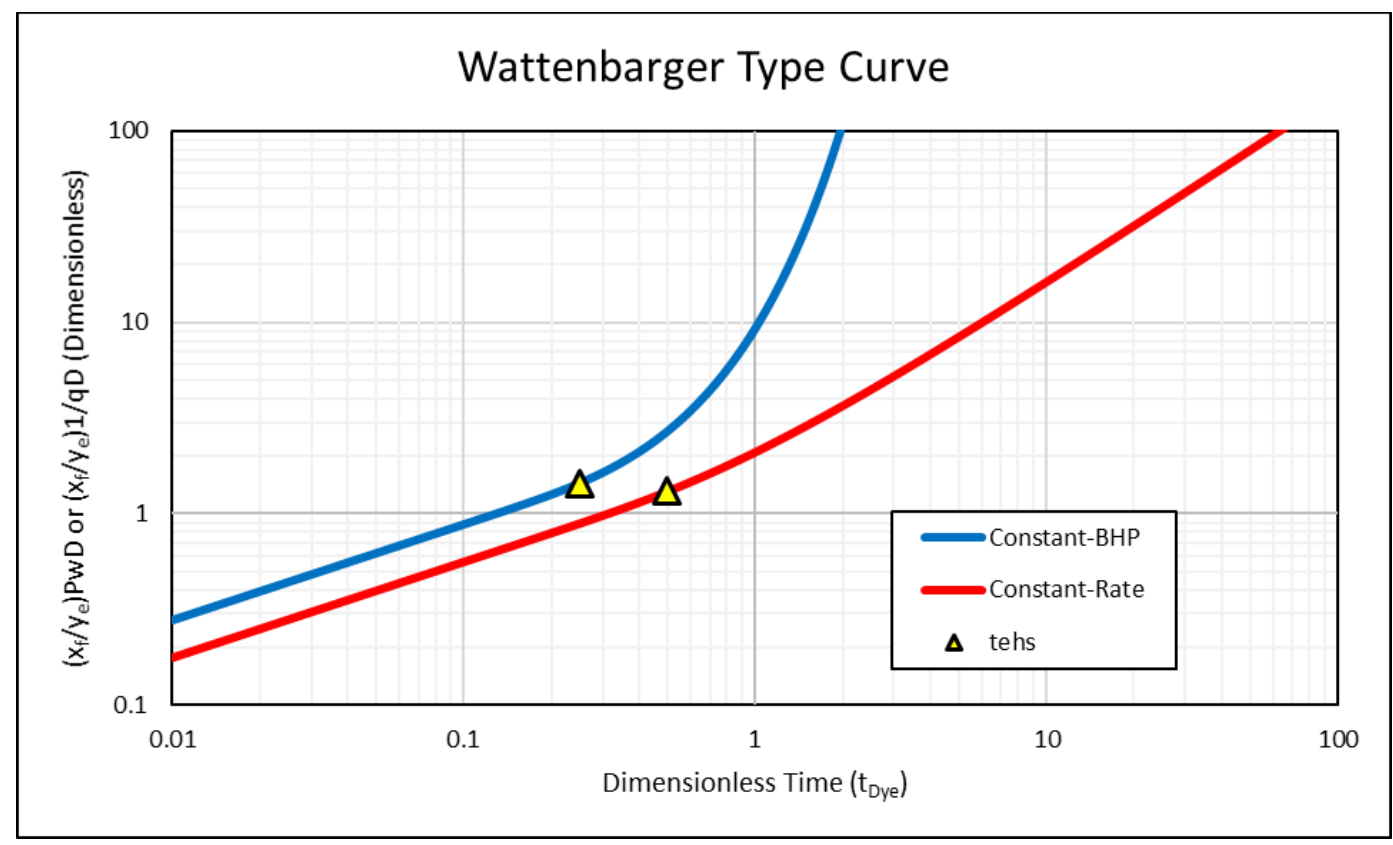

Figure 89 - Wattenbarger Type Curve (Based on Wattenbarger et al. (1998))

End of Linear Flow - Lee and Spivey (2013) 
Though Lee and Spivey (2013) does not explicitly address the end of linear flow problem, they do provide a generalized depth of investigation (DOI) definition that we included in this study. Lee and Spivey (2013) defines the depth of investigation as the distance from a pressure sink where the peak pressure disturbance occurs. More specifically, they define the depth of investigation as the distance where $\frac{d p}{d t}$ reaches its maximum value. For linear flow, this value is given in Equation (3.19).

$$
D O I=\sqrt{\frac{k t}{1896 \phi \mu c_{t}}}
$$

If we state that linear flow ends upon the onset of "strong interference" on a fracture by its nearest neighbor, we can say that the end of linear flow occurs when the DOI equals fracture spacing, $x_{s}$. Re-arrange to a form similar to previous expressions, and we have Equation (3.20), and in simplified form we have Eq.

$$
\begin{gathered}
t_{\text {elf }}=\frac{948 \phi \mu c_{t} x_{s}^{2}}{0.5 k} \\
t_{\text {elf }}=\frac{x_{s}^{2}}{2 \alpha}
\end{gathered}
$$

\section{Motivation for a New telf Expression}

Refer again to Figure 87. We already have numerous expressions for the end of linear flow, all of which seem to fall somewhere near the onset of APSS flow. The motivation for a new expression may not be obvious. 
Song et al. (2011) and Song and Ehlig-Economides (2011) both provided estimators for the end of linear flow, but show inconsistency between publications. They apply a depth of investigation concept in both publications but use the distance to a noflow boundary (half the fracture spacing) as the measured distance in their expressions. As we showed in Chapter 2, the onset of APSS flow occurs as the result of pressure disturbance on a fracture, caused by its nearest neighbors. This implies that our expression for the end of linear flow should use the distance between a fracture and its nearest neighbor, the fracture spacing, rather than the distance to the stagnation line between them.

Wattenbarger et al. (1998) provided estimators for both relevant IBC's but did not provide a mathematical basis for their estimators. They used an "eyeball" pick of the end of a half-slope on their derived type curve.

The $t_{e l f}$ expression derived from Lee and Spivey (2013) has mathematical backing, but it indicates the onset of "strong interference", not the very beginning of a flow regime.

\section{Transient Drainage Volumes}

With Figure 90 we illustrate two nearby fractures (one as a blue vertical line and one as a red vertical line), with pillbox-shaped drainage areas that have grown large enough for each fracture to draw down on its neighbor. This is a simple representation of what the end of linear flow looks like. In Figure 91 and Figure 92 we show a profile view of the same pair of fractures, with a series of dotted lines to represent the 
propagating pressure wave created by the blue fracture, to be detected by the red fracture. Figure 91 represents a constant wellbore rate case, and Figure 92 represents a constant wellbore bottom hole pressure case. Considering this system, we should define the end of linear flow as the point in time where the pressure wave from the blue fracture becomes detectable at its neighbor, the red fracture.

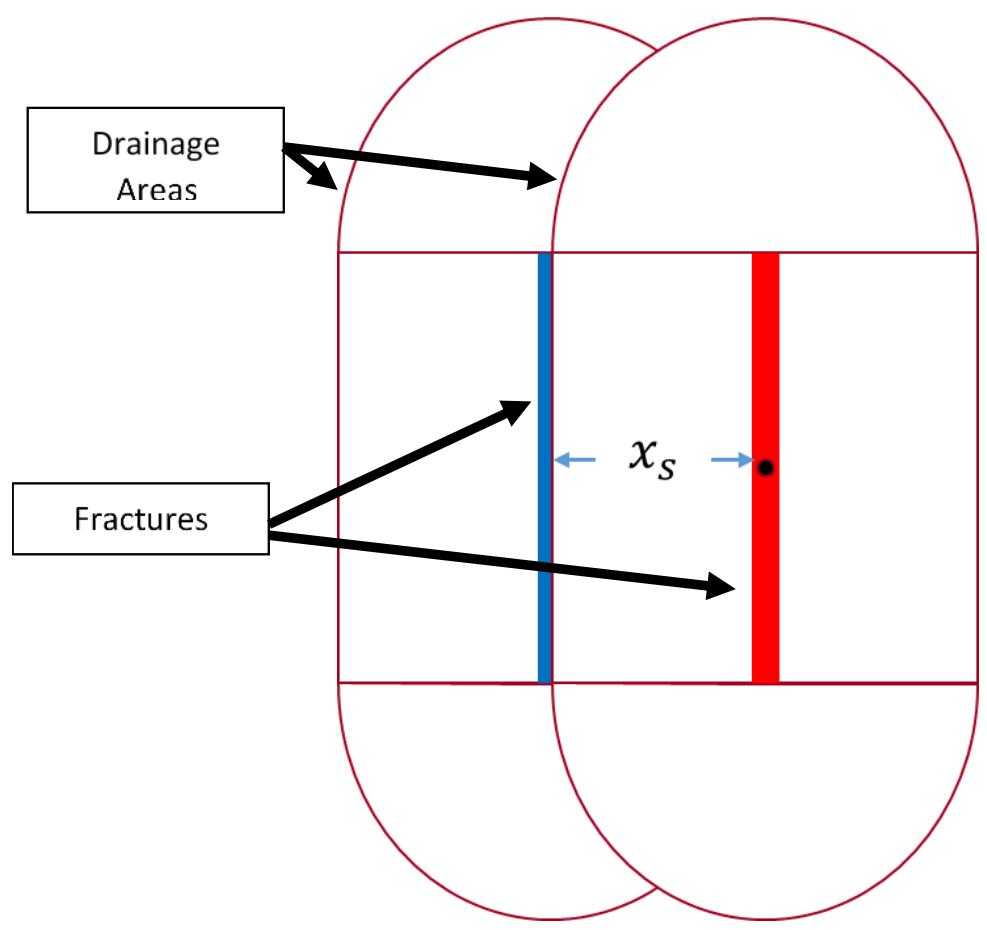

Figure 90 - Hydraulic Fracture Drainage Areas

In Chapter 2 we discussed how the asymptotic approximation of the diffusivity equation provides us with a root expression that can yield diffusivity relationships for nearly any flow geometry and inner boundary condition. With this in mind, we recall the 
base expression from the asymptotic expansion (3.22) and the expression for transient drainage volume (3.23).

$$
\begin{gathered}
c_{t} \frac{\partial p(\tau, t)}{\partial t}=\frac{1}{w(\tau)} \frac{\partial q(\tau, t)}{\partial \tau}=-\left\{\sum_{n=0}^{\infty} \tau^{n} A_{n}(t)\right\} e^{-\tau^{2} / 4 t} \\
V_{n}(t)=\int_{\tau=0}^{\tau=\infty} w(\tau) \tau^{n} e^{-\tau^{2} / 4 t} \partial \tau
\end{gathered}
$$

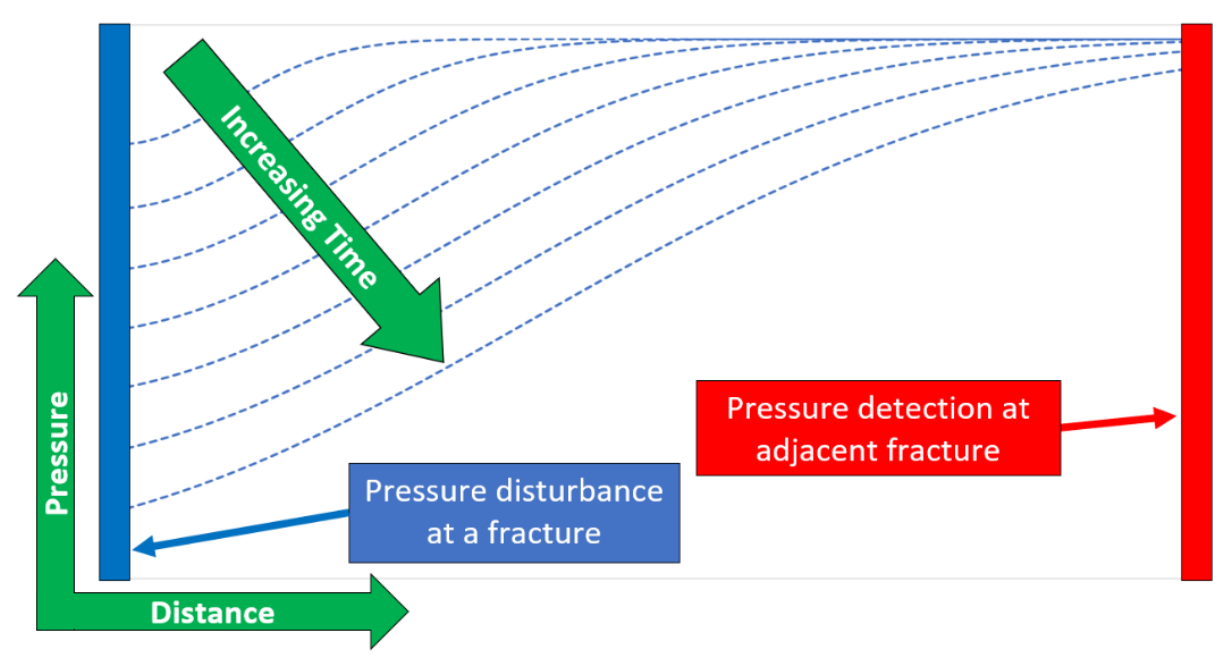

Figure 91 - Pressure propagation representation (constant rate) 


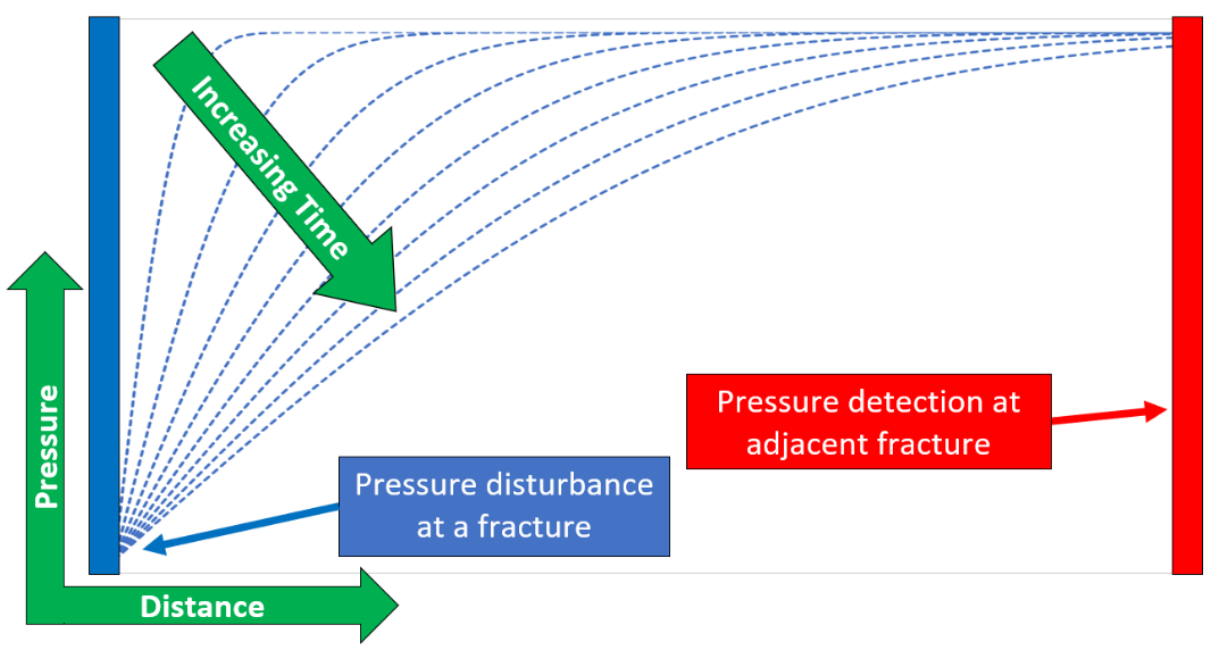

Figure 92 - Pressure propagation representation (constant BHP) Rewrite (3.23) and we have a more descriptive expression, Eq. (3.24). The well's transient drainage volume is actually an integral taken over a differential volume, $\partial V_{p}$ (Wang et al. 2018). The diffusion kernel, $e^{-\tau^{2} / 4 t}$, provides the fraction of that differential volume which contributes to the transient drainage volume of the well.

$$
V_{n}(t)=\int_{\tau=0}^{\tau=\infty} \tau^{n} e^{-\tau^{2} / 4 t} \partial V_{p}
$$

Rewrite (3.22) and we have another helpful expression, Eq. (3.25). The derivative of pressure with respect to time will vary proportionally with the diffusion kernel, $e^{-\tau^{2} / 4 t}$. Obviously, the power term, $\tau^{2} / 4 t$ (known as the Boltzmann Variable), will control this diffusion kernel.

$$
c_{t} \frac{\partial p(\tau, t)}{\partial t}=-\left\{\sum_{n=0}^{\infty} \tau^{n} A_{n}(t)\right\} e^{-\tau^{2} / 4 t}
$$

With this in mind, we can say that both the transient drainage volume and the pressure derivative of the well will vary proportionally with this diffusion kernel. Plotting the 
diffusion kernel against the square root of the Boltzmann variable shows us a dimensionless representation of any pressure wave's profile through both space and time (Figure 93). This curve illustrates where different solutions to the diffusivity equation are valid. From the wellbore ( $\tau=0$ and $\left.e^{-\tau^{2} / 4 t}=1\right)$, out to the PSS limit $(\tau / \sqrt{4 t}=0.1$, $\left.e^{-\tau^{2} / 4 t}=0.99\right)$, pseudo steady state solutions are valid. In this region, effectively all volume contributes to the well drainage volume (Wang et al. 2018). From the PSS limit to the limit of detectability $\left(\tau / \sqrt{4 t}=2, e^{-\tau^{2} / 4 t}=0.018\right)$ lies the transient region where transient solutions are valid. In the transient region, volume that is farther from the wellbore will contribute less to its drainage volume. Past that lies the initial region, where less than $1.8 \%$ of the reservoir pore volume contributes to the well's drainage volume. 


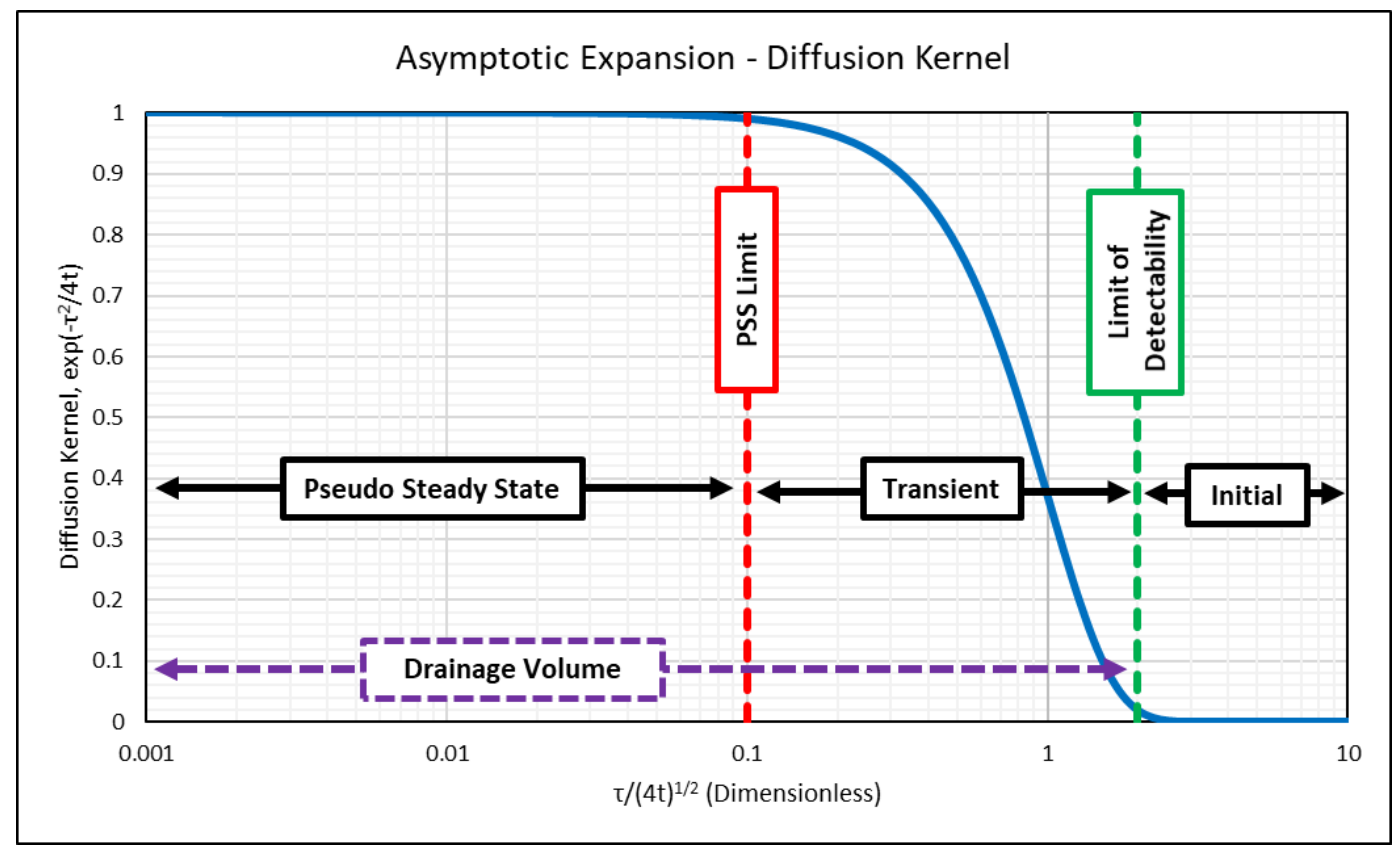

Figure 93 - Diffusion kernel spatial profile (from Malone et al. (2019)). Copyright 2019, SPE. Reproduced with permission of SPE. Further reproduction prohibited without permission.

\begin{tabular}{|ccc|}
\hline Solution & Diffusion Kernel & Boltzmann Variable \\
\hline Pseudo Steady State (PSS) & $e^{-\tau^{2} / 4 t}>0.99$ & $\frac{\tau}{\sqrt{4 t}}<0.1$ \\
\hline
\end{tabular}




\begin{tabular}{|c|c|c|}
\hline Transient & $0.99>e^{-\tau^{2} / 4 t}>0.018$ & $0.1<\frac{\tau}{\sqrt{4 t}}<2$ \\
\hline Limit of Detectability & $e^{-\tau^{2} / 4 t}=0.018$ & $\frac{\tau}{\sqrt{4 t}}=2$ \\
\hline Near Initial & $e^{-\tau^{2} / 4 t}<0.018$ & $\frac{\tau}{\sqrt{4 t}}>2$ \\
\hline
\end{tabular}

Table 4 - Diffusion Kernel and Boltzmann Variable values for asymptotic approximation solution (from Malone et al. (2019)). Copyright 2019, SPE. Reproduced with permission of SPE. Further reproduction prohibited without permission.

\section{End of Linear Flow Based on the Limit of Detectability}

\section{Theory}

The limit of detectability mentioned in Section 3.3 corresponds to the beginning of the transient drainage region as defined by the asymptotic approximation of the diffusivity equation. It represents the point in both space and time where the pressure derivative with respect to time reaches $1.8 \%$ (approximately $1 \%$ ) of its maximum value. This occurs where $\tau / \sqrt{4 t}=2$. Using the value of $\tau$ for homogeneous reservoir solutions (Equation (3.26)), we can state exactly where and when the onset of transient pressure influence begins for a single pressure sink (3.27).

$$
\begin{gathered}
\tau=\frac{r}{\sqrt{\alpha}} \\
\frac{r}{\sqrt{4 \alpha t}}=2
\end{gathered}
$$


As we mentioned in Chapter 2, fracture interference begins when the pressure pulse from one fracture becomes detectable by its nearest neighbors. Defining "detectable" as the beginning of a transient influence of one fracture on another, we can use the limit of detectability to define the onset of fracture interference and approximate pseudo steady state flow. Setting $r=x_{s}$, and $t=t_{\text {elf }}$ we have Eq. (3.28). In dimensional form, we have Eq. (3.29).

$$
\begin{gathered}
t_{\text {elf }}=\frac{x_{s}^{2}}{16 \alpha} \\
t_{\text {elf }}=\frac{948 \phi \mu c_{t} x_{s}^{2}}{4 k}
\end{gathered}
$$

Given the generalized nature of the limit of detectability, it does not depend upon flow geometry or boundary conditions, making it extremely versatile. Coincidentally, the limit of detectability is identical to Wattenbarger et al. (1998)'s end of linear flow for constant-BHP IBC's.

\section{Validation - Synthetic Case}

Taking our 2-dimensional model from Chapter 2 (reservoir properties listed in Table 5), we can compute the end of linear flow for our synthetic case. Plot this against the diagnostic plot from our constant-rate numerical simulation and we see the end of linear flow fall just before the onset of fracture interference (Figure 94).

$$
t_{\text {elf }}=\frac{948(0.06)(1)\left(2 * 10^{-5}\right)\left(50^{2}\right)}{4(0.0002)}=3555 \mathrm{hrs}
$$




\begin{tabular}{|rcl|}
\hline Reference Model Properties \\
\hline \multicolumn{3}{|c|}{ Rock \& Fluid Properties } \\
\hline Permeability, $\mathrm{k}=$ & $\mathbf{0 . 0 0 0 2}$ & $\mathrm{md}$ \\
Porosity, $\phi=$ & $\mathbf{6 \%}$ & \\
Total Compressibility, $\mathrm{c}_{\mathrm{t}}=$ & $\mathbf{2 * 1 0 ^ { - 5 }}$ & $\mathrm{psi}^{-1}$ \\
Oil Viscosity, $\mu=$ & $\mathbf{1}$ & $\mathrm{cp}$ \\
Pay Height, $\mathrm{h}=$ & $\mathbf{3 0}$ & $\mathrm{ft}$ \\
Dormation Volume Factor, $\mathrm{B}=$ & $\mathbf{1}$ & $\mathrm{rb} / \mathrm{stb}$ \\
Diffusivity, $\alpha=$ & $\mathbf{0 . 0 4 4}$ & $\mathrm{ft}{ }^{2} / \mathrm{hr}$ \\
\hline Model Fracture Properties & \\
\hline Fracture Half-Length, $\mathrm{x}_{\mathrm{f}}=$ & $\mathbf{5 0}$ & $\mathrm{ft}$ \\
Fracture Spacing, $\mathrm{x}_{\mathrm{s}}=$ & $\mathbf{5 0}$ & $\mathrm{ft}$ \\
Number of Fractures, $\mathrm{N}_{\mathrm{f}}=$ & $\mathbf{2 0}$ & $\mathrm{fractures}$ \\
Fracture Permeability, $\mathrm{k}_{\mathrm{f}}=$ & $\mathbf{1 0 0 0}$ & $\mathrm{md}$ \\
Fracture Width, $\mathrm{w}_{\mathrm{f}}=$ & $\mathbf{0 . 0 5}$ & $\mathrm{ft}$ \\
Dimensionless Frac. Cond., $\mathrm{C}_{\mathrm{rd}}=$ & $\mathbf{1 6 0 0}$ & (infinite Cond.) \\
\hline
\end{tabular}

Table 5 - Synthetic Model Properties (from Malone et al. (2019)). Copyright 2019, SPE. Reproduced with permission of SPE. Further reproduction prohibited without permission.

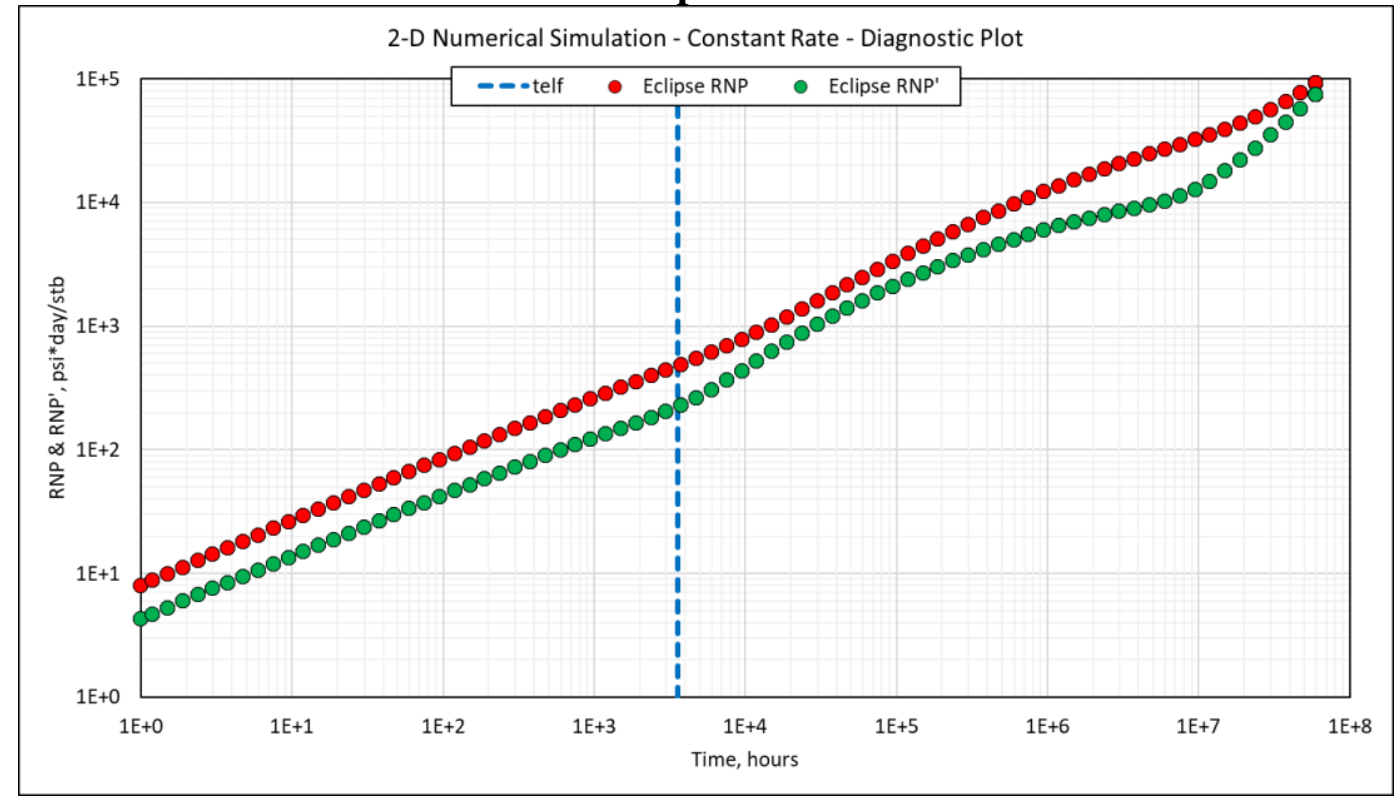

Figure 94 - End of Linear Flow - Limit of Detectability (Constant Rate Case) 
We also plot our computed value of $t_{e l f}$ against the diagnostic plot from our constant-BHP numerical simulation (Figure 95). Again, $t_{\text {elf }}$ falls just before fracture interference begins. We also have the end of linear flow in material balance time. Since we know material balance time as a function of real time, we plot the two against each other and obtain an equivalent end of linear flow in material balance time (Figure 97, Eq. (3.31))

$$
t_{\text {elf }, m b}=6554 h r s
$$

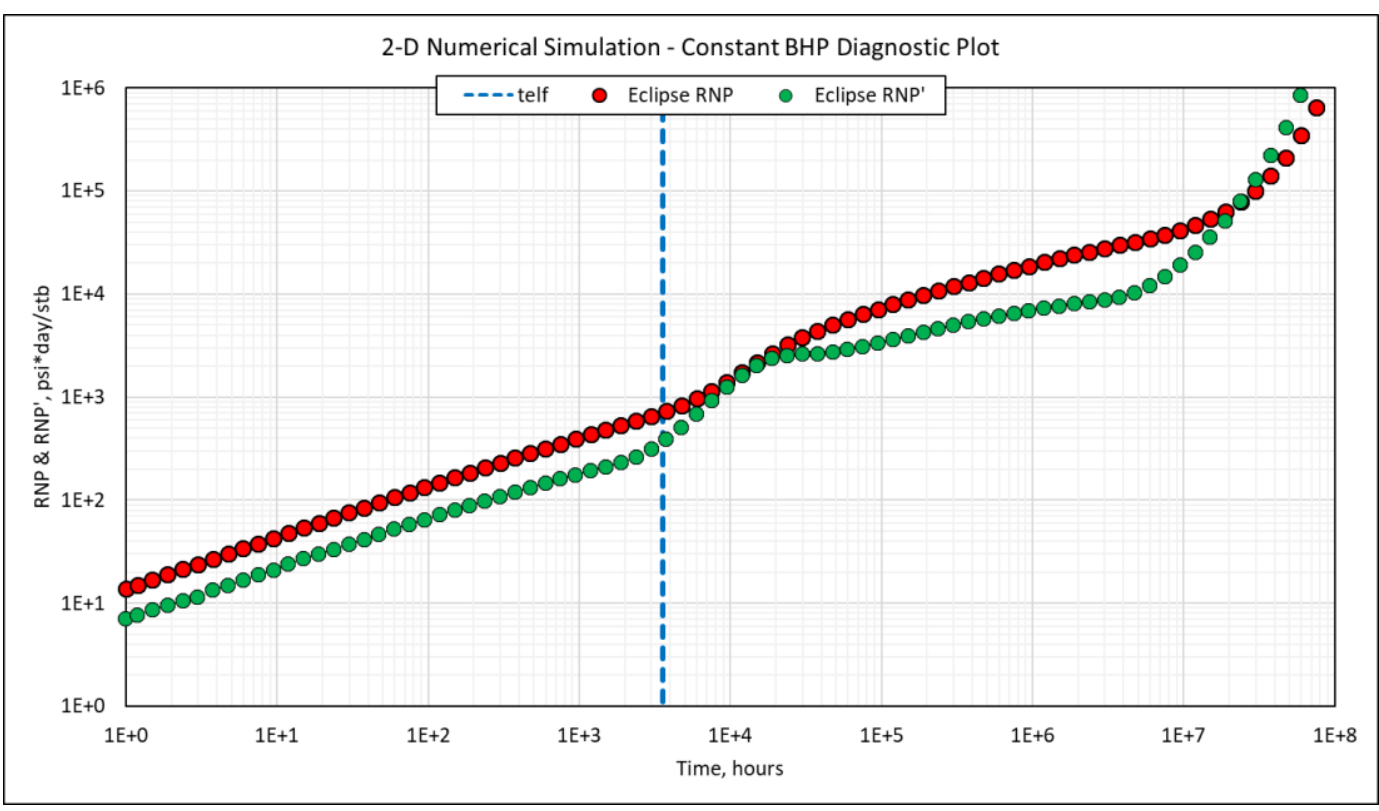

Figure 95 - End of Linear Flow - Limit of Detectability (Constant BHP Case) 


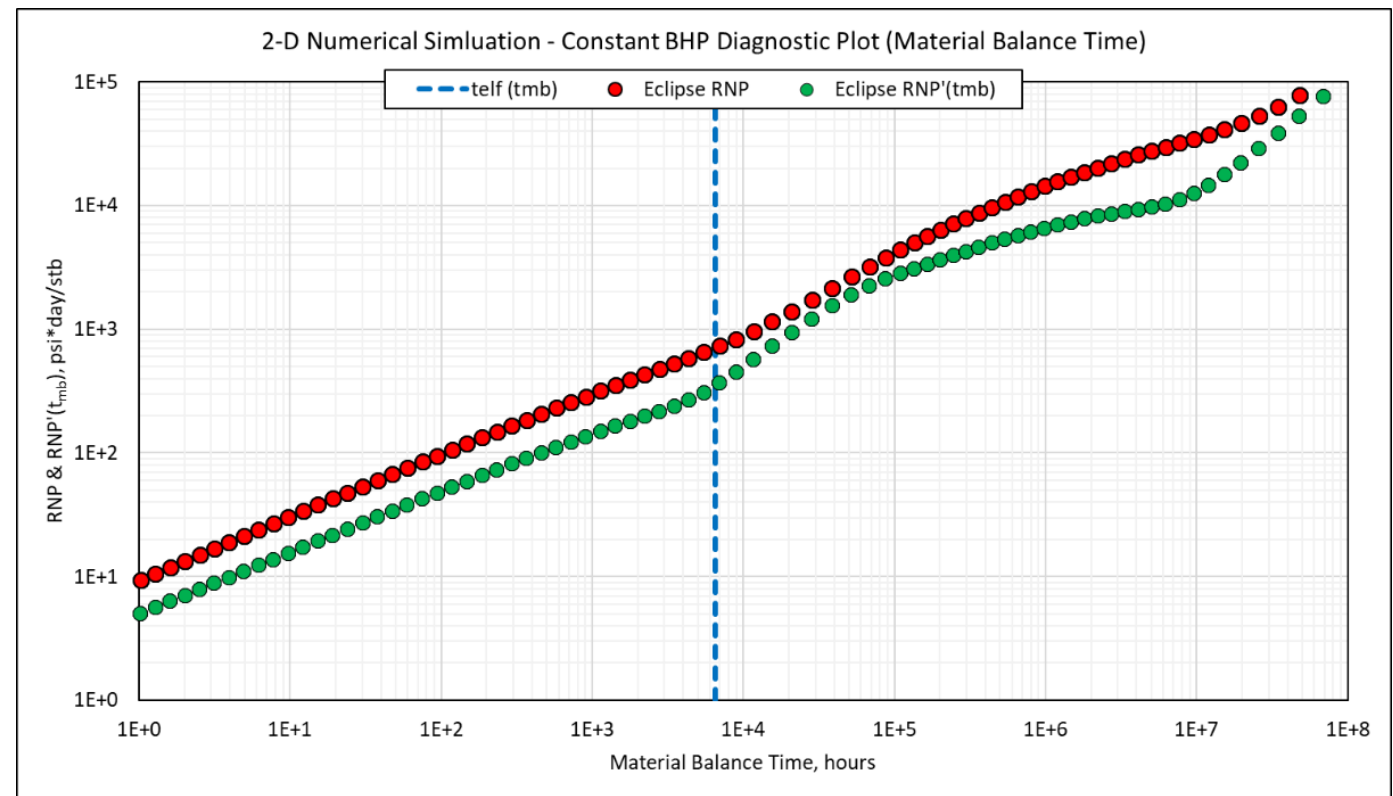

Figure 96 - End of Linear Flow - Limit of Detectability (Constant BHP Case)

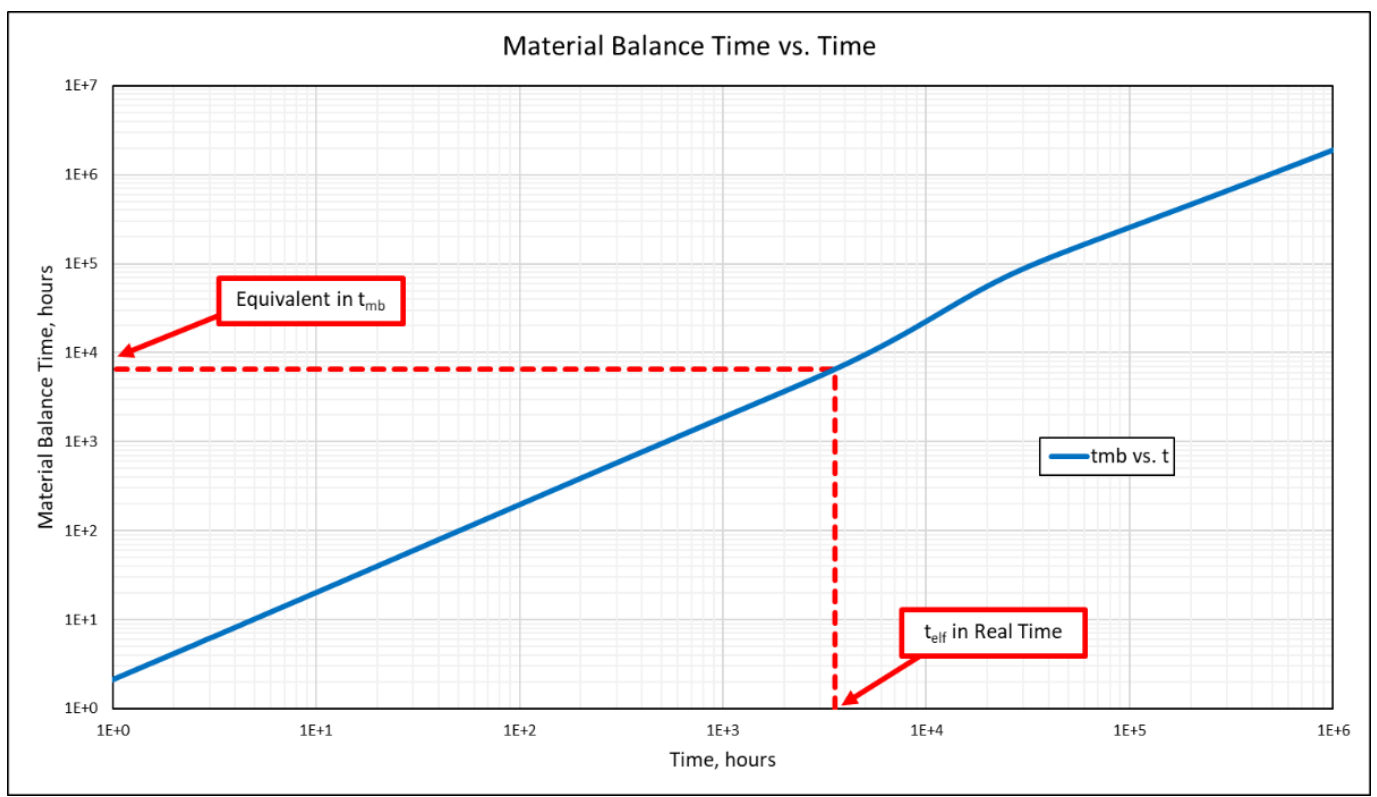

Figure 97 - End of Linear Flow conversion from real time to material balance time

Validation \& Application - Montney Field Case 
A previous study (Wang et al. 2018) discussed the estimation of permeability for a well in the Montney Field in Canada. Reservoir and well properties are listed in Table 6 and gas rate and pressure data are plotted in Figure 98. Wang et al. (2018) proposed a robust methodology for estimating permeability from production data based upon analysis of drainage volume and $w(\tau)$ curves computed through optimization. Their smoothed drainage volume and $w(\tau)$ curves work to robustly visualize fracture interference, which appears as a stagnation, or flattening, on the drainage volume curve, and as a sharp dive on its derivative, $w(\tau)$ (Figure 98). This methodology yielded a proven permeability estimate of $3.7 \mu \mathrm{D}$ through inversion of the buildup period highlighted in green in Figure 98. 


\begin{tabular}{|c|c|c|}
\hline Property & Value & Unit \\
\hline$h$ & 200 & $f t$ \\
\hline$n_{f}$ & 28 & \\
\hline$x_{f}$ & 250 & $f t$ \\
\hline$x_{s}$ & 182 & $f t$ \\
\hline$p_{i}$ & 4620 & psi \\
\hline$T_{\text {res }}$ & 190 & ${ }^{\circ} \mathrm{F}$ \\
\hline$\phi$ & 0.045 & - \\
\hline$S_{w}$ & 0.25 & - \\
\hline$L_{w}$ & 5000 & $f t$ \\
\hline \multicolumn{3}{|c|}{ Buildup Information } \\
\hline$q_{\text {last }}$ & 2867 & Mscf/day \\
\hline $\bar{p}$ & 1080 & psi \\
\hline $\bar{\mu}_{g}$ & 0.0183 & $c p$ \\
\hline $\bar{c}_{t}$ & $1.21 \times 10^{-4}$ & $p s i^{-1}$ \\
\hline $\bar{B}_{g}$ & 0.0161 & $M c f / M s c f$ \\
\hline \multicolumn{3}{|c|}{ Buildup Information } \\
\hline $\bar{p}$ & 3350 & psi \\
\hline $\bar{\mu}_{g}$ & 0.0172 & $c p$ \\
\hline $\bar{c}_{t}$ & $2.06 \times 10^{-4}$ & $p s i^{-1}$ \\
\hline
\end{tabular}

Table 6 - Montney Field Case Well \& Reservoir Properties (From Wang et al. (2018))

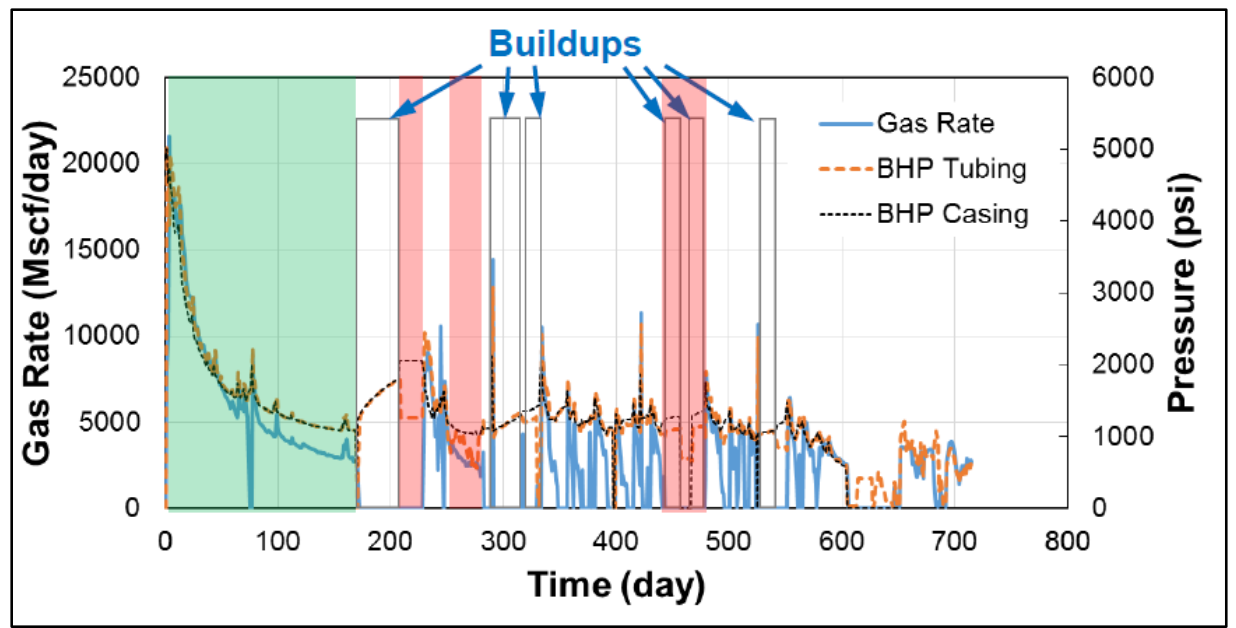

Figure 98 - Montney Field Case Production Data (From Wang et al. (2018)) 


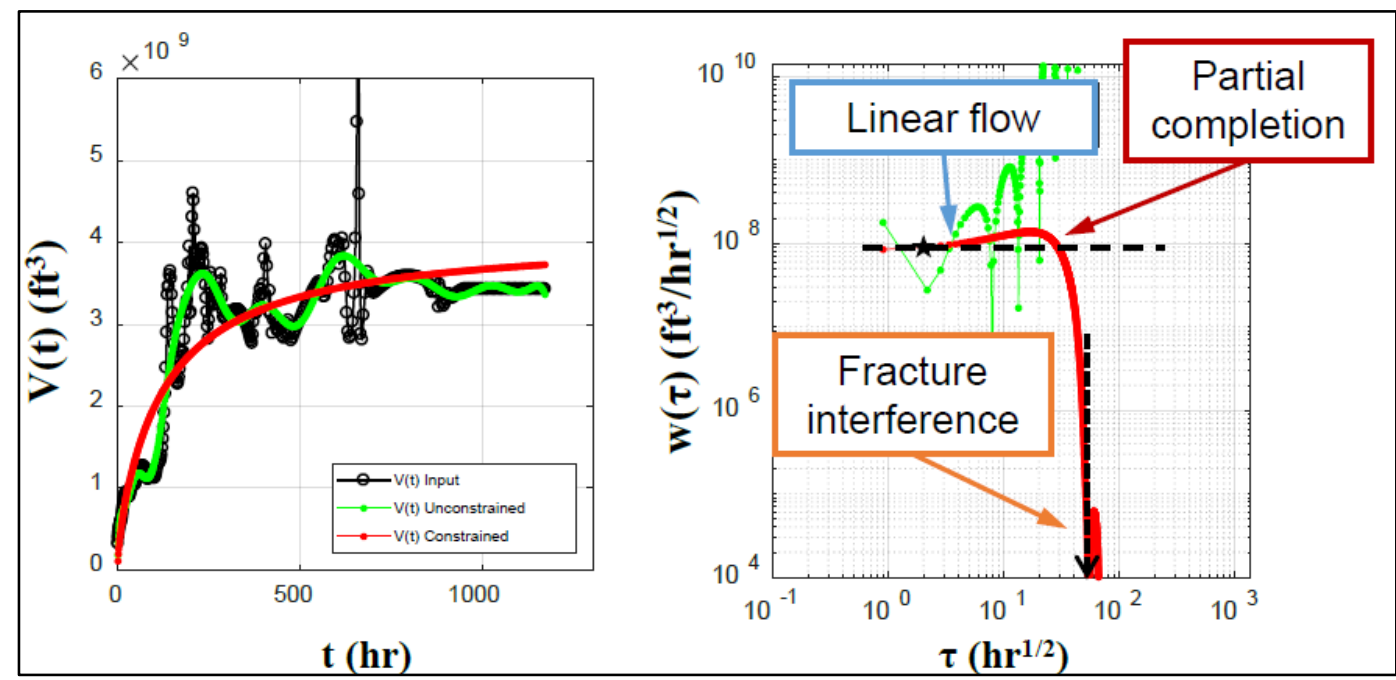

Figure 99 - Montney field case optimized drainage volume (left) and w(t) (right) curves (From Wang et al. (2018))

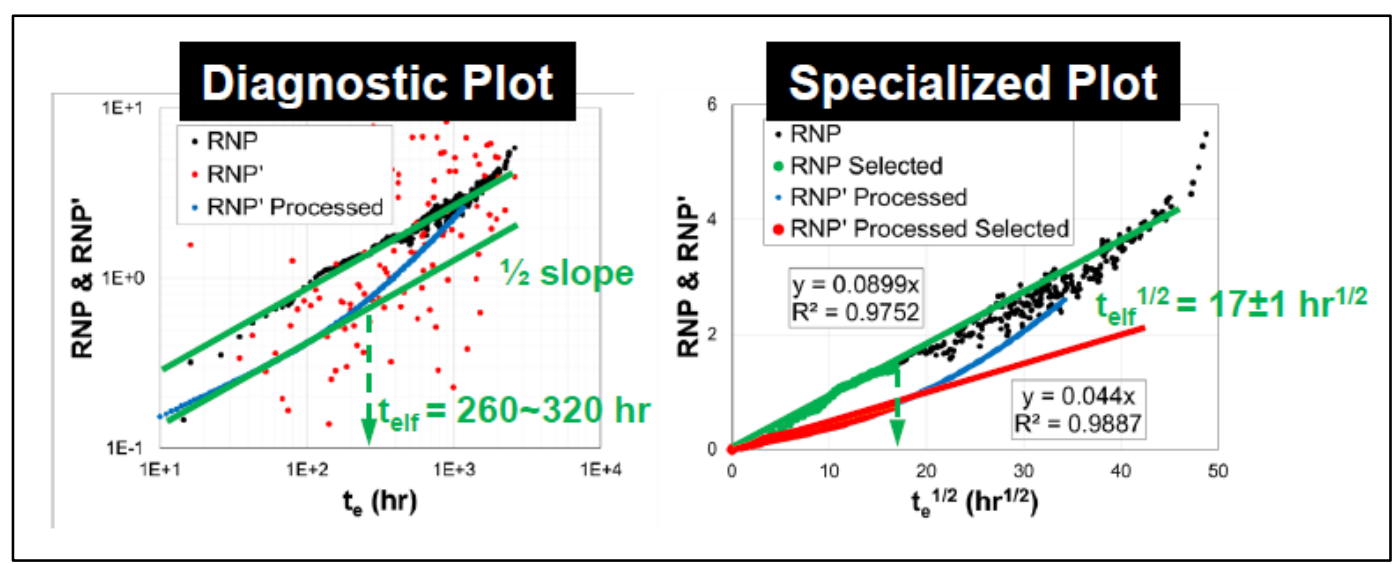

Figure 100 - Montney field case diagnostic plot (left) and specialized plot (right)

(From Wang et al. (2018))

In Figure 100 we plot the diagnostic plot (RNP \& RNP' vs. material balance time, $t_{e}$ ) and a specialized plot of the Montney field case production data. Picking the end of linear flow is easiest on a cartesian plot, and for this we use the specialized plot. 
However, picking the "break" from a half-slope is very difficult with field data (shown as points in Figure 100), so we also plot synthetic RNP and RNP' curves calculated through $w(\tau)$ inversion (described in Wang et al. (2018)). The blue RNP' curve's break from a straight-line trend on both plots indicates the end of linear flow $\left(t_{e l f}\right)$ occurs at $\sqrt{t_{e, e l f}}=17 \sqrt{h r}$, or $t_{e, e l f}=289 h r$. Convert this to regular time as in the synthetic example with the plot in Figure 101, and we have $t_{e}=331 \mathrm{hr}$ in real time.

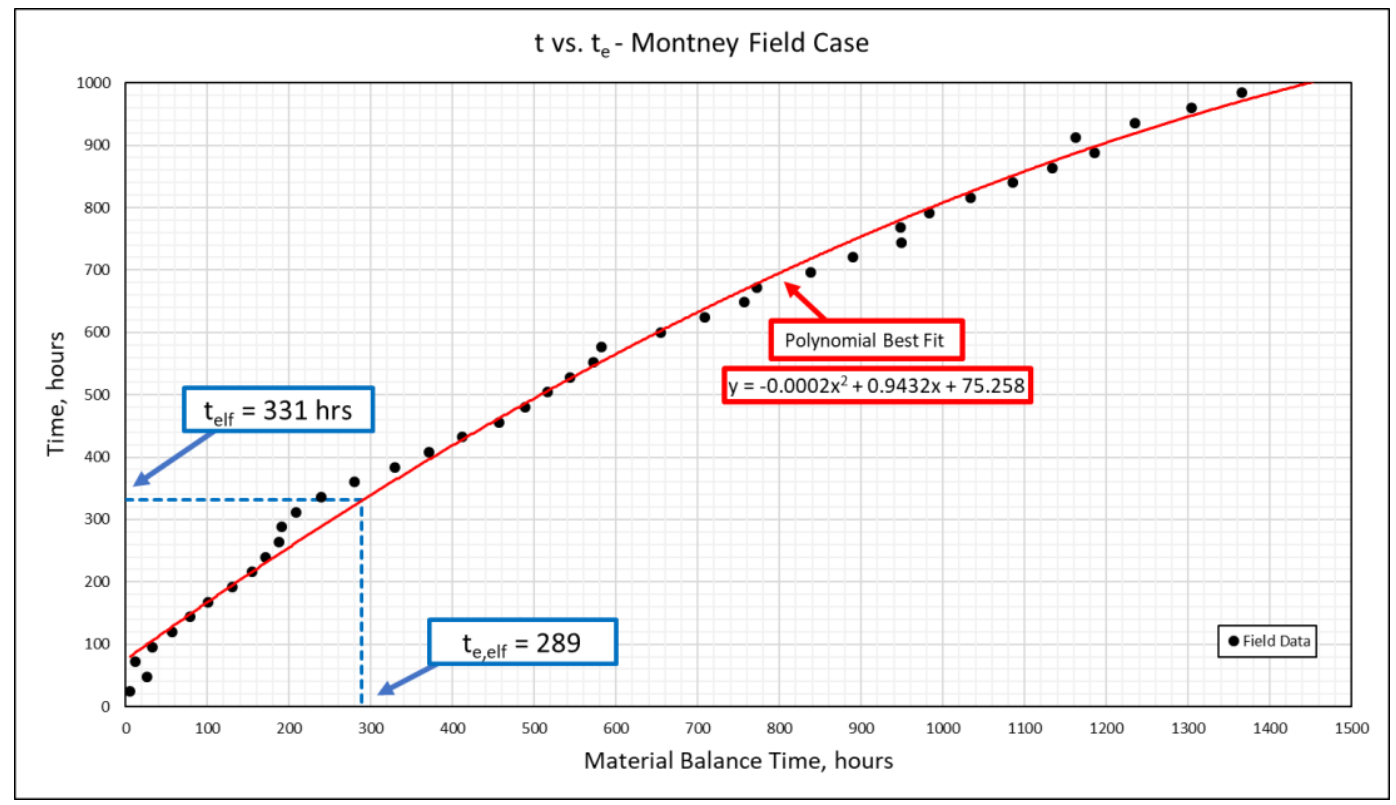

Figure 101 - Time conversion plot (after Malone et al. (2019)). Copyright 2019, SPE. Reproduced with permission of SPE. Further reproduction prohibited without permission.

Wang et al. (2018) also calculated permeability using the end of linear flow expression provided in Song and Ehlig-Economides (2011). In Table 7 we compare this value to 
what we compute using the limit of detectability and what we compute with every other equation listed in Section 3.2. Of all the reviewed expressions for the end of linear flow, the limit of detectability comes closest to matching the permeability estimate of the aforementioned $w(\tau)$ inversion-based methodology.

\begin{tabular}{|cc|}
\hline \multicolumn{2}{|c|}{$\begin{array}{c}\text { Permeability Calculation Comparison } \\
\left(\mathbf{t}_{\text {elf }}=\mathbf{3 3 1} \text { hrs) }\right.\end{array}$} \\
\hline Equation Source & $\begin{array}{c}\text { Permeability Value } \\
(\boldsymbol{\mu D})\end{array}$ \\
\hline $\begin{array}{c}\text { Song \& Economides (2011) } \\
\text { Song et al. (2011) }\end{array}$ & 0.95 \\
Wattenbarger et all (1998) \\
Constant-BHP \\
$\begin{array}{l}\text { Wattenbarger et all (1998) } \\
\text { Constant-Rate } \\
\text { Lee \& Spivey (2013) }\end{array}$ \\
$\begin{array}{l}\text { Limit of Detectability } \\
\text { w( } \tau \text { ) Inversion }\end{array}$ \\
\hline
\end{tabular}

Table 7 - Permeability Estimation Comparison (from Malone et al. (2019)). Copyright 2019, SPE. Reproduced with permission of SPE. Further reproduction prohibited without permission. 


\section{Conclusions}

In this chapter we discussed existing ambiguity in the definition of the end of transient linear flow in the context of MTFW's completed in ultra-low permeability unconventional reservoirs. We reviewed existing expressions that define the end of linear flow and explained where each method falls short. We reviewed the implications of the asymptotic approximation of the diffusivity equation and its representation of wellbore drainage volumes, and how it can relate to the definition of a pressure wave in the reservoir.

The limit of detectability, a concept that defines the front of a pressure wave as the point where it first becomes detectable, provides a robust expression that we may use to define the end of linear flow in MTFW's without dependence on boundary conditions or flow geometry. We validated our expression against a synthetic model and showed how it can be used to simply estimate the permeability of the rock matrix between fractures. Using the limit of detectability improved upon other estimations in the Montney field case and came closest to our robust $w(\tau)$ based estimation.

One obvious issue remains - picking the end of linear flow on a diagnostic plot or a specialized plot for the purposes of permeability estimation can be very difficult with noisy field data. In our Montney field case, we used smoothed data generated with the inversion algorithm described in Wang et al. (2018). In the absence of smooth field data (which is highly likely), either the same methodology or a similar methodology is recommended to obtain a recognizable "pickup" from a straight-line trend on a specialized plot. 


\section{CHAPTER IV}

\section{SUMMARY AND CONCLUSIONS}

In this document we began by presenting a framework for shale oil and gas reservoir evaluation, providing a review of recent literature in the fields of unconventional reservoir geology, petrophysics, and engineering. We focused on parameters that evaluators might find important during shale reservoir development and contextualized those parameters as they pertained to the Eagle Ford shale.

From there we took one piece of that framework - fluid flow to a multiple transverse fracture well (MTFW) and described a unique way to represent the flow to MTFW's that utilizes the principle of superposition in conjunction with the asymptotic approximation of the diffusivity equation to model the interactions between fractures during drainage. We showed through validation against a commercial simulator reference model that our method holds valid for both constant-rate applications and constant-BHP applications. Our model showed that the approximate pseudo steady state response seen in MTFW's during fracture interference is the signature of superimposed pressure drawdowns on individual fractures induced by their neighbors.

Finally, we investigated the end of transient linear flow in MTFW's and reviewed the level of ambiguity that exists in current literature. We introduced the limit of detectability, a concept founded upon the drainage volume formulation behind the asymptotic approximation of the diffusivity equation and showed how it can be used to define the end of linear flow in MTFW's. We validated our usage of the limit of detectability against both a commercial simulator reference model and a Montney shale 
field case that was presented in previous literature by Wang et al. (2018), but for which we provide an alternate interpretation.

\section{Future Work}

We recommend the following future work to expand upon the work done in this study:

1. Testing of a 3-term analytic MTFW solution - Use of a 3-term analytic MTFW solution for constant-BHP inner boundary conditions might allow the solution to achieve the non-monotonicity of the reference model.

2. Inclusion of analytic models in inversion - Inclusion of our analytic models in a production diagnostic inversion algorithm could provide a strong future project. Inversion algorithms that rely on analytic models in lieu of 3-D reservoir models see a dramatic improvement in computation speed (Moinfar et al. 2016).

3. Development of 3-dimensional analytic solutions - The solutions presented here extend only to 2-dimensional solutions. Many unconventional reservoirs have pay heights in excess of 100's of feet and thus likely violate the "full completion" assumption and would benefit from 3-dimensional modeling.

\section{Development of gas and multiphase solutions - These solutions were} developed with a single-phase, oil-only fluid assumption. Many unconventional reservoirs are gas reservoirs, and many unconventional reservoirs see multiphase flow. All unconventional reservoirs produce some water. Development of solutions that can handle these parameters would make excellent next steps. 


\section{REFERENCES}

Agarwal, A. 2011. Performance of Assisted History Matching Techniques When Utilizing Multiple Initial Geologic Models. Master of Science, Texas A\&M University.

Akkutlu, I.Y. and Fathi, E. 2012. Multiscale Gas Transport in Shales with Local Kerogen Heterogeneities. SPE Journal 17 (04): 1002-1011. DOI: 10.2118/146422-PA

Ambrose, R.J., Hartman, R.C., Diaz-Campos, M. et al. 2012. Shale Gas-in-Place Calculations Part I: New Pore-Scale Considerations. SPE Journal 17 (01): 219229. DOI: $10.2118 / 131772-P A$

Anderson, D.M., Nobakht, M., Moghadam, S. et al. 2010. Analysis of Production Data from Fractured Shale Gas Wells. Paper presented at the SPE Unconventional Gas Conference, Pittsburgh, Pennsylvania, USA. 15. Society of Petroleum Engineers. DOI: 10.2118/131787-MS.

Bello, R.O. and Wattenbarger, R.A. 2010. Multi-Stage Hydraulically Fractured Horizontal Shale Gas Well Rate Transient Analysis. Paper presented at the North Africa Technical Conference and Exhibition, Cairo, Egypt. 17. Society of Petroleum Engineers. DOI: 10.2118/126754-MS.

Blasingame, T.A. 2008. The Characteristic Flow Behavior of Low-Permeability Reservoir Systems. Paper presented at the SPE Unconventional Reservoirs Conference, Keystone, Colorado, USA. 31. Society of Petroleum Engineers. DOI: $10.2118 / 114168-\mathrm{MS}$.

Bohacs, K.M., Passey, Q.R., Rudnicki, M. et al. 2013. The Spectrum of Fine-Grained Reservoirs From. Paper presented at the International Petroleum Technology Conference, Beijing, China. 16. International Petroleum Technology Conference. DOI: 10.2523/IPTC-16676-MS.

Chen, C.-C. and Rajagopal, R. 1997. A Multiply-Fractured Horizontal Well in a Rectangular Drainage Region. SPE Journal 2 (04): 455-465. DOI: 10.2118/37072-PA 
Cipolla, C.L., Lewis, R.E., Maxwell, S.C. et al. 2011. Appraising Unconventional Resource Plays: Separating Reservoir Quality from Completion Effectiveness. Paper presented at the International Petroleum Technology Conference, Bangkok, Thailand. 27. International Petroleum Technology Conference. DOI: 10.2523/IPTC-14677-MS.

Cipolla, C.L., Lolon, E., Erdle, J.C. et al. 2009. Reservoir Modeling in Shale-Gas Reservoirs. Paper presented at the SPE Eastern Regional Meeting, Charleston, West Virginia, USA. 19. Society of Petroleum Engineers. DOI: 10.2118/125530MS.

Clarkson, C.R. 2013. Production Data Analysis of Unconventional Gas Wells: Review of Theory and Best Practices. International Journal of Coal Geology 109-110: 101-146. DOI: https://doi.org/10.1016/j.coal.2013.01.002

Clarkson, C.R., Haghshenas, B., Ghanizadeh, A. et al. 2016. Nanopores to Megafractures: Current Challenges and Methods for Shale Gas Reservoir and Hydraulic Fracture Characterization. Journal of Natural Gas Science and Engineering 31: 612-657. DOI: https://doi.org/10.1016/j.jngse.2016.01.041

Clarkson, C.R., Jordan, C.L., Ilk, D. et al. 2009. Production Data Analysis of Fractured and Horizontal Cbm Wells. Paper presented at the SPE Eastern Regional Meeting, Charleston, West Virginia, USA. 30. Society of Petroleum Engineers. DOI: 10.2118/125929-MS.

Cui, X., Bustin, A.M.M., and Bustin, R.M. 2009. Measurements of Gas Permeability and Diffusivity of Tight Reservoir Rocks: Different Approaches and Their Applications. Geofluids 9 (3): 208-223. DOI: 10.1111/j.1468-8123.2009.00244.x

Dahi Taleghani, A. and Olson, J.E. 2013. How Natural Fractures Could Affect Hydraulic-Fracture Geometry. SPE Journal 19 (01): 161-171. DOI: 10.2118/167608-PA

Dawson, W.C. 2000. Shale Microfacies-Eagle Ford Group (Cenomanian-Turonian) North-Central Texas Outcrops and Subsurface Equivalents. AAPG Bulletin 50 (2000): 607-621. DOI: 10.1306/8626c005-173b-11d7-8645000102c1865d 
Donovan, A. and Staerker, S. 2010. Stratigraphy of the Eagle Ford (Boquillas) Formation in South Texas. Gulf Coast Association of Geological Societies Transactions 60: 861-899.

Duong, A.N. 2010. An Unconventional Rate Decline Approach for Tight and FractureDominated Gas Wells. Paper presented at the Canadian Unconventional Resources and International Petroleum Conference, Calgary, Alberta, Canada. 15. Society of Petroleum Engineers. DOI: 10.2118/137748-MS.

Economides, M.J., Hill, D.A., Ehlig-Economides, C.A. et al. 2013. Petroleum Production Systems: Pearson Education, Inc. Original edition. ISBN.

Ehlig-Economides, C.A. 1992. Computation of Test Area of Investigation in Nonradial Geometries. Paper presented at the European Petroleum Conference, Cannes, France. Society of Petroleum Engineers. DOI: 10.2118/25020-MS.

EIA. 2011. World Shale Gas Resources: An Initial Assessment of 14 Regions Outside the United States. https://www.eia.gov/analysis/studies/worldshalegas/archive/2011/pdf/fullreport 2011.pdf.

EIA. 2013. Technically Recoverable Shale Oil and Shale Gas Resources: An Assessment of 137 Shale Formations in 41 Countries Outside the United States. https://www.eia.gov/analysis/studies/worldshalegas/archive/2013/pdf/fullreport 2013.pdf.

Ferrill, D.A., McGinnis, R.N., Morris, A.P. et al. 2014. Control of Mechanical Stratigraphy on Bed-Restricted Jointing and Normal Faulting: Eagle Ford Formation, South-Central Texasmechanical Stratigraphy, Faults and Fractures in the Eagle Ford of Texas. AAPG Bulletin 98 (11): 2477-2506. DOI: $10.1306 / 08191414053$

Fetkovich, M.J. 1980. Decline Curve Analysis Using Type Curves. Journal of Petroleum Technology 32 (06): 1065-1077. DOI: 10.2118/4629-PA

Gale, J.F.W., Laubach, S.E., Olson, J.E. et al. 2014. Natural Fractures in Shale: A Review and New Observationsnatural Fractures in Shale: A Review and New Observations. AAPG Bulletin 98 (11): 2165-2216. DOI: 10.1306/08121413151 
Gidley, J.L., Holditch, S.A., Nierode, D.E. et al. 1990. Recent Advances in Hydraulic Fracturing. Spe Monograph Series: Society of Petroleum Engineers. Original edition. ISBN 978-1-55563-020-1.

Hartman, R.C., Ambrose, R.J., Akkutlu, I.Y. et al. 2011. Shale Gas-in-Place Calculations Part Ii - Multicomponent Gas Adsorption Effects. Paper presented at the North American Unconventional Gas Conference and Exhibition, The Woodlands, Texas, USA. 17. Society of Petroleum Engineers. DOI: 10.2118/144097-MS.

Hentz, T.F., and Ruppel, S.C. 2010. Regional Lithostratigraphy of the Eagle Ford Shale: Maverick Basin to East Texas Basin. Gulf Coast Association of Geological Societies Transactions 60: 325-337.

Hinton, D.D. 2012. The Seventeen-Year Overnight Wonder: George Mitchell and Unlocking the Barnett Shale. Journal of American History 99 (1): 229-235. DOI: 10.1093/jahist/jas064

Jarvie, D., J. Hill, R., Ruble, T. et al. 2007. Unconventional Shale-Gas Systems: The Mississippian Barnett Shale of North-Central Texas as One Model for Thermogenic Shale-Gas Assessment Original edition. ISBN.

Jarvie, D.M. 2012a. Shale Resource Systems for Oil and Gas: Part 1 - Shale-Gas Resource Systems. In Shale Reservoirs - Giant Resources for the 21 st Century, ed. Breyer, J.A.: AAPG Memoir. 97.

Jarvie, D.M. 2012b. Shale Resource Systems for Oil and Gas: Part 2 - Shale-Oil Resource Systems. In Shale Reservoirs - Giant Resources for the 21 st Century, ed. Breyer, J.A.: AAPG Memoir. 97.

Javadpour, F. 2009. Nanopores and Apparent Permeability of Gas Flow in Mudrocks (Shales and Siltstone). Journal of Canadian Petroleum Technology 48 (08): 1621. DOI: $10.2118 / 09-08-16-\mathrm{DA}$

King, M.J., Wang, Z., and Datta-Gupta, A. 2016. Asymptotic Solutions of the Diffusivity Equation and Their Applications. Paper presented at the SPE Europec featured at 78th EAGE Conference and Exhibition, Vienna, Austria. Society of Petroleum Engineers. DOI: 10.2118/180149-MS. 
Ko, L.T., Loucks, R.G., Ruppel, S.C. et al. 2017. Origin and Characterization of Eagle Ford Pore Networks in the South Texas Upper Cretaceous Shelf. AAPG Bulletin 101 (3): 387-418. DOI: 10.1306/08051616035

Kulkarni, K.N., Datta-Gupta, A., and Vasco, D.W. 2001. A Streamline Approach for Integrating Transient Pressure Data into High-Resolution Reservoir Models. SPE Journal 6 (03): 273-282. DOI: 10.2118/74135-PA

Lee, J., Rollins, J.B., Spivey, J.P. et al. 2003. Pressure Transient Testing. Spe Textbook Series ; V. 9; Spe Textbook Series ; V. 9. Richardson, Tex. :: Society of Petroleum Engineers. Original edition. ISBN 15556309959781555630997.

Lee, W.J. and Spivey, J.P. 2013. Applied Well Test Interpretation. Spe Textbook Series: Society of Petroleum Engineers. Original edition. ISBN 978-1-61399-307-1.

Li, J., Yu, W., and Wu, K. 2018. Analyzing the Impact of Fracture Complexity on Well Performance and Wettability Alteration in Eagle Ford Shale. Paper presented at the SPE/AAPG/SEG Unconventional Resources Technology Conference, Houston, Texas, USA. 15. Unconventional Resources Technology Conference. DOI: 10.15530/URTEC-2018-2899349.

Lock, B.E., Peschier, L., and Whitcomb, N. 2010. The Eagle Ford (Boquillas Formation) of Val Verde County, Texas - a Window on the South Texas Play. Gulf Coast Association of Geological Societies Transatcions 60: 419-434.

Loucks, R.G., Reed, R.M., Ruppel, S.C. et al. 2009. Morphology, Genesis, and Distribution of Nanometer-Scale Pores in Siliceous Mudstones of the Mississippian Barnett Shale. Journal of Sedimentary Research 79 (12): 848-861. DOI: $10.2110 /$ jsr.2009.092

Malone, A., Wang, Z., and King, M.J. 2019. Characterization of Multiple Transverse Fracture Wells Using the Asymptotic Approximation of the Diffusivity Equation. Paper presented at the SPE Europec featured at the 81st EAGE Conference and Exhibition, London, United Kingdom. 28. Society of Petroleum Engineers.

Mayerhofer, M.J., Lolon, E., Warpinski, N.R. et al. 2010. What Is Stimulated Reservoir Volume? SPE Production \& Operations 25 (01): 89-98. DOI: 10.2118/119890PA 
Moinfar, A., Erdle, J.C., and Patel, K. 2016. Comparison of Numerical Vs Analytical Models for Eur Calculation and Optimization in Unconventional Reservoirs. Paper presented at the SPE Argentina Exploration and Production of Unconventional Resources Symposium, Buenos Aires, Argentina. 21. Society of Petroleum Engineers. DOI: 10.2118/180974-MS.

Mullen, J. 2010. Petrophysical Characterization of the Eagle Ford Shale in South Texas. Paper presented at the Canadian Unconventional Resources and International Petroleum Conference, Calgary, Alberta, Canada. 19. Society of Petroleum Engineers. DOI: 10.2118/138145-MS.

Nelson, P.H. 2009. Pore-Throat Sizes in Sandstones, Tight Sandstones, and Shales. AAPG Bulletin 93 (3): 329-340. DOI: 10.1306/10240808059

Palacio, J.C. and Blasingame, T.A. 1993. Decline-Curve Analysis Using Type Curves-Analysis of Gas Well Production Data. Paper presented at the SPE Joint Rocky Mountain Regional and Low Permeability Reservoirs Symposium, Denver, CO. Society of Petroleum Engineers.

Passey, Q.R., Bohacs, K., Esch, W.L. et al. 2010. From Oil-Prone Source Rock to GasProducing Shale Reservoir - Geologic and Petrophysical Characterization of Unconventional Shale Gas Reservoirs. Paper presented at the International Oil and Gas Conference and Exhibition in China, Beijing, China. 29. Society of Petroleum Engineers. DOI: 10.2118/131350-MS.

Pommer, M. and Milliken, K. 2015. Pore Types and Pore-Size Distributions across Thermal Maturity, Eagle Ford Formation, Southern Texaspores across Thermal Maturity, Eagle Ford. AAPG Bulletin 99 (9): 1713-1744. DOI: $10.1306 / 03051514151$

Rickman, R., Mullen, M.J., Petre, J.E. et al. 2008. A Practical Use of Shale Petrophysics for Stimulation Design Optimization: All Shale Plays Are Not Clones of the Barnett Shale. Paper presented at the SPE Annual Technical Conference and Exhibition, Denver, Colorado, USA. 11. Society of Petroleum Engineers. DOI: 10.2118/115258-MS.

Rogner, H.H. 1997. An Assessment of World Hydrocarbon Resources. Annual Review of Energy and the Environment 22 (1): 217-262. DOI: 10.1146/annurev.energy.22.1.217 
Russell, B. and Freeborn, R. 2012. A Practical Guide to Unconventional Petroleum Evaluation. Paper presented at the SPE Asia Pacific Oil and Gas Conference and Exhibition, Perth, Australia. 16. Society of Petroleum Engineers. DOI: 10.2118/158867-MS.

Samandarli, O., Valbuena, E., and Economides, C. 2012. Production Data Analysis in Unconventional Reservoirs with Rate- Normalized Pressure (Rnp): Theory, Methodology, and Applications. Paper presented at the SPE Americas Unconventional Resources Conference, Pittsburgh, Pennsylvania USA. Society of Petroleum Engineers. DOI: 10.2118/155614-MS.

Sondergeld, C.H., Ambrose, R.J., Rai, C.S. et al. 2010a. Micro-Structural Studies of Gas Shales. Paper presented at the SPE Unconventional Gas Conference, Pittsburgh, Pennsylvania, USA. 17. Society of Petroleum Engineers. DOI: 10.2118/131771MS.

Sondergeld, C.H., Newsham, K.E., Comisky, J.T. et al. 2010b. Petrophysical Considerations in Evaluating and Producing Shale Gas Resources. Paper presented at the SPE Unconventional Gas Conference, Pittsburgh, Pennsylvania, USA. 34. Society of Petroleum Engineers. DOI: 10.2118/131768-MS.

Song, B., Economides, M.J., and Ehlig-Economides, C.A. 2011. Design of Multiple Transverse Fracture Horizontal Wells in Shale Gas Reservoirs. Paper presented at the SPE Hydraulic Fracturing Technology Conference, The Woodlands, Texas, USA. Society of Petroleum Engineers. DOI: 10.2118/140555-MS.

Song, B. and Ehlig-Economides, C.A. 2011. Rate-Normalized Pressure Analysis for Determination of Shale Gas Well Performance. Paper presented at the North American Unconventional Gas Conference and Exhibition, The Woodlands, Texas, USA. Society of Petroleum Engineers. DOI: 10.2118/144031-MS.

Sun, J. and Schechter, D. 2016. Pressure-Transient Characteristics of Fractured Horizontal Wells in Unconventional Shale Reservoirs with Complex Fracture Networks. Paper presented at the SPE Eastern Regional Meeting, Canton, Ohio, USA. 20. Society of Petroleum Engineers. DOI: 10.2118/184060-MS.

Sun, J., Schechter, D., and Rui, Z. 2016. Numerical Investigation of the Effect of Nonuniform Fracture Aperture of Eagle Ford Outcrop Maps on Production Performance. Paper presented at the SPE Annual Technical Conference and 
Exhibition, Dubai, UAE. 22. Society of Petroleum Engineers. DOI: 10.2118/181624-MS.

Tian, Y., Ayers, W.B., and McCain, W.D., Jr. 2013. The Eagle Ford Shale Play, South Texas: Regional Variations in Fluid Types, Hydrocarbon Production and Reservoir Properties. Paper presented at the International Petroleum Technology Conference, Beijing, China. 12. International Petroleum Technology Conference. DOI: 10.2523/IPTC-16808-MS.

Virieux, J., Flores-Luna, C., and Gibert, D. 1994. Asymptotic Theory for Diffusive Electromagnetic Imaging. Geophysical Journal International 119 (3): 857-868. DOI: $10.1111 / \mathrm{j} .1365-246 X .1994 . t b 04022 . x$

Wang, F.P. and Reed, R.M. 2009. Pore Networks and Fluid Flow in Gas Shales. Paper presented at the SPE Annual Technical Conference and Exhibition, New Orleans, Louisiana. 8. Society of Petroleum Engineers. DOI: 10.2118/124253-MS.

Wang, S., Feng, Q., Javadpour, F. et al. 2015. Oil Adsorption in Shale Nanopores and Its Effect on Recoverable Oil-in-Place. International Journal of Coal Geology 147148: 9-24. DOI: https://doi.org/10.1016/j.coal.2015.06.002

Wang, S., Javadpour, F., and Feng, Q. 2016. Molecular Dynamics Simulations of Oil Transport through Inorganic Nanopores in Shale. Fuel 171: 74-86. DOI: https://doi.org/10.1016/j.fuel.2015.12.071

Wang, Z., Malone, A., and King, M.J. 2018. Quantitative Production Analysis and Eur Prediction from Unconventional Reservoirs Using a Data-Driven Drainage Volume Formulation. Paper presented at the European Conference on the Mathematics of Oil Recovery, Barcelona, Spain.

Warpinski, N.R., Mayerhofer, M.J., Vincent, M.C. et al. 2008. Stimulating Unconventional Reservoirs: Maximizing Network Growth While Optimizing Fracture Conductivity. Paper presented at the SPE Unconventional Reservoirs Conference, Keystone, Colorado, USA. 19. Society of Petroleum Engineers. DOI: 10.2118/114173-MS.

Wasaki, A. and Akkutlu, I.Y. 2015. Permeability of Organic-Rich Shale. SPE Journal 20 (06): 1384-1396. DOI: 10.2118/170830-PA 
Wattenbarger, R.A., El-Banbi, A.H., Villegas, M.E. et al. 1998. Production Analysis of Linear Flow into Fractured Tight Gas Wells. Paper presented at the SPE Rocky Mountain Regional/Low-Permeability Reservoirs Symposium, Denver, Colorado. Society of Petroleum Engineers. DOI: 10.2118/39931-MS.

Wu, K. and Olson, J.E. 2016. Numerical Investigation of Complex Hydraulic-Fracture Development in Naturally Fractured Reservoirs. SPE Production \& Operations 31 (04): 300-309. DOI: 10.2118/173326-PA

Yao Tian, W.B.A., and William D. McCain, Jr. 2013. Regional Analysis of Stratigraphy, Reservoir Characteristics, and Fluid Phases in the Eagle Ford Shale, South Texas. Gulf Coast Association of Geological Societies Transactions 62: 471-483.

Yu, S., Lee, W.J., Miocevic, D.J. et al. 2013. Estimating Proved Reserves in Tight/Shale Wells Using the Modified Sepd Method. Paper presented at the SPE Annual Technical Conference and Exhibition, New Orleans, Louisiana, USA. 15. Society of Petroleum Engineers. DOI: 10.2118/166198-MS.

Zhang, Y., Yang, C., King, M.J. et al. 2013. Fast-Marching Methods for Complex Grids and Anisotropic Permeabilities: Application to Unconventional Reservoirs. Paper presented at the SPE Reservoir Simulation Symposium, The Woodlands, Texas, USA. 21. Society of Petroleum Engineers. DOI: 10.2118/163637-MS.

Zou, C. 2017. Unconventional Petroleum Geology (Second Edition): Elsevier. Original edition. ISBN 978-0-12-812234-1. 